Florida International University

FIU Digital Commons

FIU Electronic Theses and Dissertations

University Graduate School

$11-4-2019$

\title{
Regulation of Telomerase Activity by Rab5 Guanine Nucleotide Exchange Factors
}

Patric J. Hambleton

Florida International University, phamblet@fiu.edu

Follow this and additional works at: https://digitalcommons.fiu.edu/etd

Part of the Cancer Biology Commons, and the Cell Biology Commons

\section{Recommended Citation}

Hambleton, Patric J., "Regulation of Telomerase Activity by Rab5 Guanine Nucleotide Exchange Factors" (2019). FIU Electronic Theses and Dissertations. 4346.

https://digitalcommons.fiu.edu/etd/4346

This work is brought to you for free and open access by the University Graduate School at FIU Digital Commons. It has been accepted for inclusion in FIU Electronic Theses and Dissertations by an authorized administrator of FIU Digital Commons. For more information, please contact dcc@fiu.edu. 


\title{
FLORIDA INTERNATIONAL UNIVERSITY
}

Miami, Florida

\section{REGULATION OF TELOMERASE ACTIVITY BY RAB5 GUANINE NUCLEOTIDE EXCHANGE FACTORS}

A dissertation submitted in partial fulfillment of the

$$
\text { requirements for the degree of }
$$

DOCTOR OF PHILOSOPHY

\author{
in \\ BIOLOGY
}

by

Patric Hambleton 
To: Dean Michael R. Heithaus

College of Arts, Sciences and Education

This dissertation, written by Patric Hambleton, and entitled Regulation of Telomerase Activity by Rab5 Guanine Nucleotide Exchange Factors, having been approved in respect to style and intellectual content, is referred to you for judgement.

We have read this dissertation and recommend that it be approved.

$\begin{array}{r}\hline \text { Watson Lees } \\ \hline \text { John Makemson } \\ \hline \text { Fernando Noriega } \\ \hline \text { Xiaotang Wang } \\ \hline \text { Manuel A. Barbieri, Major Professor }\end{array}$

Date of Defense: November 4, 2019

The dissertation of Patric Hambleton is approved.

Dean Michael R. Heithaus

College of Arts, Sciences and Education

Andrés G. Gil

Vice President for Research and Economic Development and

Dean of the University Graduate School

Florida International University, 2019 
(C) Copyright 2019 by Patric Hambleton

All rights reserved. 


\section{DEDICATION}

This work is dedicated to my family without whom none of this would have been possible. Thank you to my mother, Theresa Hambleton, for her constant support and encouragement. Thank you for always being a great role model and instilling in me the values of hard work, dedication, and perseverance. She has always been there through the good times and bad, and I will be eternally grateful for a lifetime of love, wisdom, and sacrifice. Thank you to my sisters, Jennifer Hambleton and Mary Hambleton, for their support over the years and for always being there to talk, laugh, and create great memories. Thank you to my late grandmother, Dorothy Kennedy, for always providing a helping hand and for caring for my sisters and I for so many years. My success in life is owed to my family. 


\section{ACKNOWLEDGMENTS}

A very special thank you to my mentor, Dr. Manuel A. Barbieri, for his guidance and advice while completing this work. He welcomed me into his laboratory as a nontraditional graduate student and always gave me the freedom to explore my scientific curiosities. Thank you for always pushing me to excel and for providing me the resources to be successful. Thank you to my dear friend, Dr. Brenda Luna, whose encouragement and technical assistance were invaluable. Finally, thank you to Florida International University for the financial support in the form of a Biomedical Research Initiative award (NIGMS RISE R25 GM061347) that helped to make this research possible. 


\section{ABSTRACT OF THE DISSERTATION \\ REGULATION OF TELOMERASE ACTIVITY BY RAB5 GUANINE NUCLEOTIDE \\ EXCHANGE FACTORS}

by

Patric Hambleton

Florida International University, 2019

Miami, Florida

Professor Manuel A. Barbieri, Major Professor

Many cancerous cells display abnormalities in the signal transduction pathways responsible for responding to extracellular growth factors. Growth factors, such as insulinlike growth factor I, represent a major class of mitogenic ligands that can initiate the mitogen activated protein kinase (MAPK) pathway. The role of the MAPK pathway in transducing growth signals to the interior of the cell and subsequently stimulating cell growth and proliferation is highlighted by the fact that roughly one quarter of all human tumors contain mutant forms of Ras proteins. Ras interference 1 (Rin1) is involved in key steps of receptor mediated endocytosis and can potentially moderate signaling through the MAPK pathway. The possible connection between Rin1, an effector of the active form of Ras, and subsequent telomerase gene expression and activity is of particular interest as telomerase is active in roughly $85 \%$ of all cancers. In this study, we determine the effect of the expression of Rin 1 on cellular proliferation as well as on telomerase gene expression and activity in several different human cancer cells. MDA-MB 231, MCF7, and MCF-12A breast cell lines expressing Rin1 exhibit reduced levels of proliferation of up to $31 \%$ following exposure to the IGF-1 growth factor. Telomerase activity and gene expression 
were also reduced by factors of up to 1.5 and 2.55 fold in the three breast cell lines studied when compared to control cells. Similarly, overexpression of Rin1 in human Yusik melanoma cells leads to reduced telomerase activity. Telomerase activity was determined by the telomeric repeat amplification protocol (TRAP) assay while gene expression was measured by RT-qPCR. Furthermore, our observations suggest that overexpression of the Rin1 Y561F mutant and Rin1 delta splice variant in MDA-MB 231 cells results in increased proliferation and telomerase activity in these cancer cells when compared to control cells. The MDA-MB 231 cells expressing wild type Rin1 displayed lowered levels of phosphorylation for the p-44/42 (ERK), STAT3, and Ets2 transcription factors. Additionally, overexpression of the C-terminus region of Rin1 in these cells greatly reduced ERK phosphorylation. In summary, Rin1 may play a novel tumor suppressor role in modulating signaling through the Ras/MAPK pathway upon IGF-1 stimulation. 


\section{TABLE OF CONTENTS}

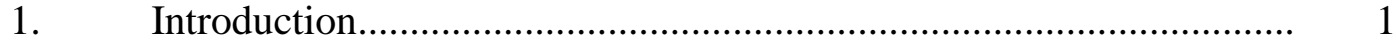

1.1 The Role of Telomerase in Cancer Cells............................................ 1

1.2 The Role of Endocytosis in Cancer Cell Migration............................. 32

1.3 The Mitogen Activated Protein Kinase Pathway and Cancer.............. 39

$1.4 \quad$ Specific Aims............................................................................. 53

$1.5 \quad$ References.............................................................................. 55

2. The Effect of Rin1 on Cellular Proliferation as well as on Telomerase Gene Expression and Activity in Breast Cancer

Cells

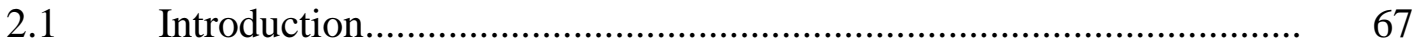

$2.2 \quad$ Results.......................................................................... 76

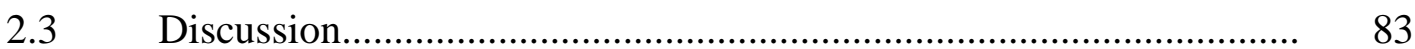

2.4 Materials and Methods..................................................................... 88

$2.5 \quad$ References................................................................................... 101

3. The Effect of Rin1 Constructs on Telomerase Protein Expression and Activity in Breast Cancer Cells.................................................. 126

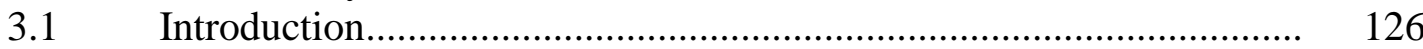

$3.2 \quad$ Results................................................................................. 134

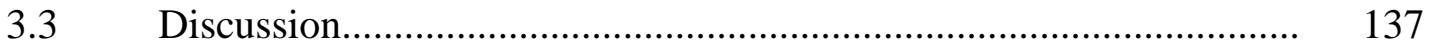

3.4 Materials and Methods................................................................. 141

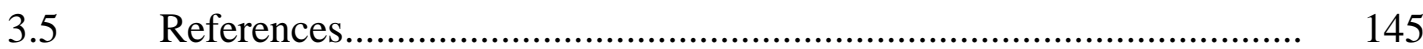

4. The Effect of Rin1 on Telomerase Activity in Human Glioblastoma and Melanoma Cells.......................................................................... 156

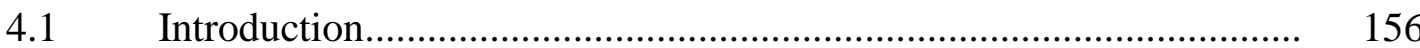

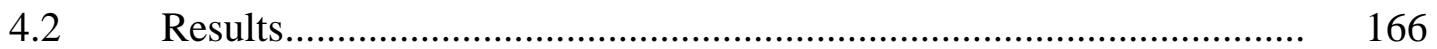

$4.3 \quad$ Discussion.............................................................................. 167

4.4 Materials and Methods................................................................... 169

$4.5 \quad$ References............................................................................... 170

5. Conclusions and Future Work............................................................ 179

VITA 


\section{LIST OF TABLES}

TABLE

PAGE

1.1 Descriptions of key terms.

65

2.1 Average cycle threshold $(\mathrm{Ct})$ values of the qPCR amplification of telomerase activity products for the serial dilution of a HeLa cell telomerase positive control sample. All experiments were conducted in duplicate.

2.2 Cycle threshold values $(\mathrm{Ct})$ for the $\mathrm{qPCR}$ products of telomerase activity in MDA-MB 231 cell lines following stimulation with 100 $\mathrm{ng} / \mathrm{mL}$ of IGF-1 (+) for 24 hours or no stimulation (-). All experiments were conducted in duplicate.

2.3 Cycle threshold values $(\mathrm{Ct})$ for the RT-qPCR of telomerase gene expression in MDA-MB 231 cell lines following stimulation with $100 \mathrm{ng} / \mathrm{mL}$ of IGF-1 (+) for 24 hours or no stimulation (-). All experiments were conducted in duplicate.

2.4 Cycle threshold values $(\mathrm{Ct})$ for the qPCR products of telomerase activity in MCF-12A cell lines following stimulation with 100 $\mathrm{ng} / \mathrm{mL}$ of IGF-1 (+) for 24 hours or no stimulation (-). All experiments were conducted in duplicate.

2.5 Cycle threshold values $(\mathrm{Ct})$ for the RT-qPCR of telomerase gene expression in MCF-12A cell lines following stimulation with 100 $\mathrm{ng} / \mathrm{mL}$ of IGF-1 (+) for 24 hours or no stimulation (-). All experiments were conducted in duplicate.

2.6 Cycle threshold values $(\mathrm{Ct})$ for the $\mathrm{qPCR}$ products of telomerase activity in MCF7 cell lines following stimulation with $100 \mathrm{ng} / \mathrm{mL}$ of IGF-1 (+) for 24 hours or no stimulation (-). All experiments were conducted in duplicate.

2.7 Cycle threshold values (Ct) for the RT-qPCR of telomerase gene expression in MCF7 cell lines following stimulation with 100 $\mathrm{ng} / \mathrm{mL}$ of IGF-1 (+) for 24 hours or no stimulation (-). All experiments were conducted in duplicate.

2.8 The effect of Rin1 expression on cellular proliferation, telomerase gene expression, and telomerase activity in different breast cell lines. 
3.1 Cycle threshold values $(\mathrm{Ct})$ for the qPCR of telomerase activity in MDA-MB 231 Rin 1 construct cell lines following stimulation with $100 \mathrm{ng} / \mathrm{mL}$ of IGF-1 (+) for 24 hours or no stimulation (-). Rin1 Y561F and Rin1 delta cells display 1.04 and 1.01 fold increases in telomerase activity compared to GFP cells. Rin1 R2N and Rin1 $\mathrm{R} 3 \mathrm{C}$ cells display 1.06 and 1.11 fold decreases in telomerase activity compared to GFP cells.

4.1 Cycle threshold values $(\mathrm{Ct})$ for the $\mathrm{qPCR}$ products of telomerase activity in U87 MG cell lines following stimulation with 100 $\mathrm{ng} / \mathrm{mL}$ of IGF-1 (+) for 24 hours or no stimulation (-). All experiments were conducted in duplicate.

4.2 Cycle threshold values $(\mathrm{Ct})$ for the $\mathrm{qPCR}$ products of telomerase activity in U118 MG cell lines following stimulation with 100 $\mathrm{ng} / \mathrm{mL}$ of IGF-1 (+) for 24 hours or no stimulation (-). All experiments were conducted in duplicate.

4.3 Cycle threshold values $(\mathrm{Ct})$ for the $\mathrm{qPCR}$ products of telomerase activity in Yusik cell lines following stimulation with $100 \mathrm{ng} / \mathrm{mL}$ of IGF-1 (+) for 24 hours or no stimulation (-). All experiments were conducted in duplicate. 


\section{LIST OF FIGURES}

FIGURE

PAGE

1.1 Diagram of the proposed model for the moderating influence of Rin1 on signaling through the Ras/MAPK pathway. The binding of a growth factor such as IGF-1 to its receptor on the plasma membrane activates Ras and subsequently the MAPK pathway. Activation of the MAPK pathway in turn activates various nuclear transcription factors involved in telomerase expression. Rin1 competes with Raf for binding to activated Ras.

1.2 Rin1 domains. SH2 binds phospho-tyrosine residues. Vps9 acts as a guanine nucleotide exchange factor for Rab5. RA binds to activated Ras. Proline rich binds to SH3 domains.

2.1 Lentiviral vector used to create the MDA-MB 231 cell line expressing Rin1. The Rin1 gene sequence is inserted directly downstream of the CMV promoter. An identical vector with the GFP gene sequence inserted was used to create the MDA-MB 231 cell line expressing GFP. Also labeled are the various elements necessary for lentivirus production and cell selection.

2.2 Figure 2.2: A) Confocal micrograph image of MDA-MB 231 cells expressing Rin1. Rin1 tends to localize near the plasma membrane (red arrows). B) Light micrograph image illustrating the clumped growth pattern of MDA-MB 231 cells expressing Rin1 (red circle).

2.3 Western blot image of the A) MDA-MB 231 GFP cell line expressing GFP and endogenous levels of Rin1. B) MDA-MB 231 cell line overexpressing Rin1.

The effect of cell number on cellular proliferation in serum exposed MDA-MB 231 cells. Error bars represent the standard error of the mean.

2.5 Regression analysis of the effect of cell number on cellular proliferation in serum exposed MDA-MB 231 cells. 
2.6 The effect of IGF-1 concentration on cellular proliferation in MDA-MB 231 cells expressing GFP or Rin1. Values represent the percent increase in cellular proliferation over an unstimulated $(0$ $\mathrm{ng} / \mathrm{mL}$ ) control. All experiments were conducted in triplicate. 108 Error bars represent standard error of the mean.

2.7 The effect of IGF-1 stimulation $(100 \mathrm{ng} / \mathrm{mL})$ on cellular proliferation of non-transduced MDA-MB 231 cells. Values represent the percent increase normalized to the serum free/no IGF-1 treatment group. All experiments were conducted in triplicate. Error bars represent the standard error of the mean.

2.8 The effect of IGF-1 stimulation $(100 \mathrm{ng} / \mathrm{mL})$ on cellular proliferation of MDA-MB 231 cells expressing GFP. Values represent the percent increase normalized to the serum free/no IGF-1 treatment group. All experiments were conducted in triplicate. Error bars represent the standard error of the mean.

The effect of IGF-1 stimulation (100 $\mathrm{ng} / \mathrm{mL})$ on cellular proliferation of MDA-MB 231 cells expressing Rin1. Values represent the percent increase normalized to the serum free/no IGF-1 treatment group. All experiments were conducted in triplicate. Error bars represent the standard error of the mean.

Telomerase activity assay demonstrating the presence of telomeric repeat amplification protocol PCR products. 1.25 bp DNA ladder. 2. HeLa cell telomerase positive control. 3. HeLa cell heat treated telomerase negative control. Red box is the 36 base pair internal control.

Amplification curves of the qPCR telomerase activity products for the serial dilution of a HeLa cell telomerase positive control sample. Red circle is undiluted sample, green triangle is $10 \mathrm{X}$ dilution, orange square is $100 \mathrm{X}$ dilution, blue cross is $1,000 \mathrm{X}$ dilution, and brown diamond is buffer only.

PAGE gel analysis of the telomerase activity qPCR TRAP products for the MDA-MB 231 cell lines following stimulation with $100 \mathrm{ng} / \mathrm{mL}$ of IGF-1 (+) for 24 hours or no stimulation (-). 1. 25 bp ladder. 2. Telomerase positive control. 3. Telomerase heat treated negative control. 4. GFP (+) IGF-1. 5. GFP (-) IGF-1. 6. Rin1 (+) IGF-1. 7. Rin1 (-) IGF-1. 8. Buffer. Red boxes indicate reduced telomerase activity in cells expressing Rin1 when compared to cells expressing GFP following IGF-1 stimulation. 
2.13 PAGE gel analysis of the RT-qPCR GAPDH and telomerase gene expression products in the MDA-MB 231 cell lines following stimulation with $100 \mathrm{ng} / \mathrm{mL}$ of IGF-1 (+) for 24 hours or no stimulation (-).

2.14 Western blot image of the A) MCF-12A GFP cell line expressing GFP and endogenous levels of Rin1. B) MCF-12A cell line overexpressing Rin1.

2.15 The effect of IGF-1 stimulation (100 ng/mL) on cellular proliferation of non-transduced MCF-12A cells. Values represent the percent increase normalized to the serum free/no IGF-1 treatment group. All experiments were conducted in triplicate. Error bars represent the standard error of the mean.

The effect of IGF-1 stimulation (100 ng/mL) on cellular proliferation of MCF-12A cells expressing GFP. Values represent the percent increase normalized to the serum free/no IGF-1 treatment group. All experiments were conducted in triplicate. Error bars represent the standard error of the mean.

The effect of IGF-1 stimulation (100 ng/mL) on cellular proliferation of MCF-12A cells expressing Rin1. Values represent the percent increase normalized to the serum free/no IGF-1 treatment group. All experiments were conducted in triplicate. Error bars represent the standard error of the mean.

Western blot image of the A) MCF7 GFP cell line expressing GFP and endogenous levels of Rin1. B) MCF7 cell line overexpressing Rin1.

The effect of IGF-1 stimulation (100 ng/mL) on cellular proliferation of non-transduced MCF7 cells. Values represent the percent increase normalized to the serum free/no IGF-1 treatment group. All experiments were conducted in triplicate. Error bars represent the standard error of the mean.

The effect of IGF-1 stimulation (100 ng/mL) on cellular proliferation of MCF7 cells expressing GFP. Values represent the percent increase normalized to the serum free/no IGF-1 treatment group. All experiments were conducted in triplicate. Error bars represent the standard error of the mean. 
2.21 The effect of IGF-1 stimulation (100 ng/mL) on cellular proliferation of MCF7 cells expressing Rin1. Values represent the percent increase normalized to the serum free/no IGF-1 treatment group. All experiments were conducted in triplicate. Error bars represent the standard error of the mean.

122

3.1 Lentiviral vector used to create the MDA-MB 231 cell line expressing Rin1 Y561F. The Rin1 Y561F gene sequence is inserted directly downstream of the CMV promoter. An identical vector with the Rin1 delta gene sequence inserted was used to create the MDA-MB 231 cell line expressing Rin1 delta. Also labeled are the various elements necessary for lentivirus production and cell selection.

3.2 A) Confocal micrograph image of MDA-MB 231 cells expressing Rin1 Y561F. Rin1 Y561F tends to localize throughout the cytoplasm. B) Light micrograph image illustrating the even growth pattern of MDA-MB 231 cells expressing Rin1 Y561F.

3.3 A) Confocal micrograph image of MDA-MB 231 cells expressing Rin1 delta. Rin1 delta tends to localize throughout the cytoplasm. B) Light micrograph image illustrating the even growth pattern of MDA-MB 231 cells expressing Rin1 delta.

3.4 Western blot image of the A) MDA-MB 231 Rin1 Y561F cell line expressing Rin1 Y561F. B) MDA-MB 231 Rin delta cell line overexpressing Rin1 delta.

3.5 Expression of the carboxyl terminal region of Rin1 in the MDAMB 231 R3C cell line.

3.6 Effect of IGF-1 concentration on telomerase protein expression in MDA-MB 231 GFP cells. Cells were stimulated with 0,10 , or 50 $\mathrm{ng} / \mathrm{mL}$ of IGF-1 for 12 hours.

3.7 Effect of Rin1 constructs on telomerase protein expression in MDA-MB 231 cells. Cells were stimulated with $50 \mathrm{ng} / \mathrm{mL}$ of IGF1 for 24 hours. Values represent the percent increase normalized to GFP cells. 
Effect of Rin1 amino (R2N) and carboxyl (R3C) terminal regions on the ratio of $\mathrm{p}-\mathrm{ERK} / \mathrm{ERK}$ in MDA-MB 231 GFP cells following IGF-1 stimulation (100 ng/mL) for 24 hours.

3.9 Western blot image of the effect of Rin1 on the expression and phosphorylation status of various proteins in the MDA-MB 231 GFP and Rin1 cell lines following IGF-1 induction $(25 \mathrm{ng} / \mathrm{mL})$ for the times indicated (minutes).

3.10 Western blot image of the effect of Rin1 on the expression and phosphorylation status of various proteins in the MDA-MB 231 amino (R2N) and carboxyl (R3C) cell lines following IGF-1 induction $(25 \mathrm{ng} / \mathrm{mL})$ for the times indicated (minutes).

3.11 The effect of IGF-1 concentration on cellular proliferation in MDA-MB 231 cells expressing Rin1 Y561F or Rin1 delta. Values represent the percent increase in cellular proliferation over an unstimulated $(0 \mathrm{ng} / \mathrm{mL})$ control. All experiments were conducted in triplicate. Error bars represent standard error of the mean.

Lentiviral vector used to create the U87 MG, U118 MG, and Yusik cell lines expressing Rin1. The Rin1 gene sequence is inserted directly downstream of the CMV promoter. An identical vector with the GFP gene sequence inserted was used to create the U87 MG, U118 MG, and Yusik cell lines expressing GFP. Also labeled are the various elements necessary for lentivirus production and cell selection.

4.2 Western blot image of the A) U87 MG glioblastoma cell line expressing GFP and endogenous levels of Rin1. B) U87 MG glioblastoma cell line overexpressing Rin1.

4.3 Light micrograph images of the A) U87 MG GFP cells growing in a clumped, irregular pattern and B) U87 MG Rin1 cells growing in an even, uniform pattern.

Western blot image of the A) U118 MG glioblastoma cell line expressing GFP and endogenous levels of Rin1. B) U118 MG glioblastoma cell line overexpressing Rin1.

4.5 Western blot image of the A) Yusik melanoma cell line expressing GFP and endogenous levels of Rin1. B) Yusik melanoma cell line overexpressing Rin1. 


\section{CHAPTER 1}

\section{Introduction}

\subsection{The Role of Telomerase in Cancer Cells}

Cancer is a disorder of the cell cycle. The hallmark of any malignant tumor is the abnormal and excessive proliferation of cells which begins to impair the normal functioning of a tissue or organ (Buseman et al., 2012). In healthy cells, the events and timing of the cell cycle are precisely regulated so that cells only divide under appropriate circumstances in a controlled manner. The tight regulation of the events of the cell cycle is largely accomplished through the use of key checkpoints $\left(\mathrm{G}_{1}, \mathrm{G}_{2}, \mathrm{M}\right)$ in which a cell can temporarily halt progression through the cycle to assess if crucial events have occurred properly to that point (Buseman et al., 2012). Many types of cancer cells have lost the ability to arrest the cell cycle at one or more of the important checkpoints. Thus, these cells progress atypically through the cell cycle regardless of aberrations which would otherwise cause healthy cells to not divide. Any number of abnormal events may occur both internally and externally that result in the loss of a checkpoint (Buseman et al., 2012). The most common cause of many of the classic abnormalities associated with cancer cells is mutation. A change(s) in the DNA sequence of critical cell cycle regulation genes is usually the main culprit. For instance, mutations in the DNA of tumor suppressor genes such as p53 or the genes which code for proteins involved in cell signaling are quite prevalent in a variety of different cancers (Chen \& Chen, 2011).

\subsection{A. Telomere Structure and Function}

Telomeres are unique regions of DNA located at the very tips of eukaryotic chromosomes. These regions of DNA typically consist of a highly repetitive sequence 
(TTAGGG in vertebrates) and are tasked with a number of important functions related to chromosome structure and integrity. Principal among these, is the role of telomeres in acting as a sort of molecular clock during cell division. The repetitive DNA at the telomeres acts as a buffer which slows the erosion of important regions of DNA during successive rounds of cell division. As most somatic cells progress though the cell cycle, a loss of approximately $150 \mathrm{bp}$ of DNA occurs at the telomeric regions with each round of division (Dwyer et al., 2007; Liu, 1999) This loss is a result of the inability of DNA polymerase enzymes to complete DNA synthesis on the lagging daughter DNA strand during replication (S phase). As a consequence, the telomeres become progressively shorter with each round of cell division and the amount of DNA loss serves to indicate the number of divisions (Gomez et al., 2012). The telomeres are eventually eroded to a critical threshold length at which time they become unstable and a multitude of cellular DNA damage mechanisms are activated to initiate replicative senescence or apoptosis (Dwyer et al., 2007). Erosion of telomeres has important meaning for aging and tumorigenesis.

Structurally, telomeric DNA is observed to exist as large duplex loops known as t-loops (Liu, 1999). A t-loop occurs when the single stranded 3' overhang left behind after DNA synthesis is looped back around and inserted into the double stranded telomeric repeat region. The formation of t-loops helps to protect the telomeres from further DNA damage, fusion, and recombination by various cellular DNA repair enzymes, and may also be necessary for proper replication of the telomeric DNA (Liu, 1999). A six subunit protein complex known as "shelterin" aids in the formation of t-loops and is also thought to be involved in regulating telomerase activity. Telomere repeat factor 2 (TRF2), a protein essential to t-loop formation, is part of the shelterin complex along with TRF1, POT1, 
RAP1, TIN2, and TPP1. Collectively, these telomere binding proteins, among others, are shed during repeated rounds of cell division as a consequence of telomere shortening and may serve to interact with signaling molecules. Reduction of telomere length may also promote the expression of genes which are sensitive to telomere length, a phenomenon which has been reported for the p53 tumor suppressor gene in mice (Liu, 1999).

\subsection{B. Telomerase Structure and Function}

While most somatic cells will enter senescence or undergo apoptosis after roughly 50 to 70 rounds of cell division, there are certain populations of cells which can avoid these fates. These include embryonic stem cells, adult somatic stem cells, and germ cells. A ribonucleoprotein with reverse transcriptase ability known as telomerase is responsible for preventing the shortening of telomeres in cells that express it. The enzyme in humans consists primarily of a 445 nucleotide RNA template (hTR) which directs the extension and addition of the telomeric repeat sequence (TTAGGG), and an accompanying 1132 amino acid (120 kDa) catalytic subunit with reverse transcriptase activity (hTERT). The RNA subunit is encoded by a single gene and is integral to the function of the enzyme (Liu, 1999).

One of the major causes for the rapid rate of cell division observed in multiple types of cancer is the overexpression of telomerase. While telomerase expression is suppressed in the vast majority of normal somatic cells, its expression and activity can be quite significant in many types of cancerous cells (Liu, 1999). In fact, overexpression of telomerase is observed in approximately $85 \%$ of all human cancers and often confers an immortal phenotype to those cells which overexpress it (Liu, 1999). The deregulation of cell signaling pathways which results in the abnormal expression of telomerase is a hallmark 
of most cancers and is therefore of great clinical importance. The idea that cancer cells abnormally express telomerase is logical given that cancer cells undergo rapid and aberrant cell division, and thus may rely on telomerase expression to prevent the excessive shortening of chromosomes which would otherwise induce senescence or apoptosis.

\subsection{Signal Transduction Pathways and the Regulation of Telomerase Activity}

The unique role that telomerase plays in cancer cells has been widely reported. In fact, roughly $80 \%$ to $90 \%$ of all cancer cells display high levels of telomerase activity (Buseman et al., 2012; Chen \& Chen, 2011; Gomez et al., 2012). Therefore, telomerase is an ideal target for cancer therapies because the enzyme is not expressed in the vast majority of normal somatic cells and can be targeted with some specificity only in the cancerous cells. Telomerase activity in cancer cells appears to enable these cells to maintain the length of their telomeres above the critical threshold for triggering senescence or apoptosis. As a consequence, cancer cells tend to escape the normal cellular mechanisms of senescence and apoptosis, which are designed to prevent any further cell division in cells that have unstable and/or damaged chromosomes (Bernardes de Jesus \& Blasco, 2013).

Like many proteins, telomerase activity is regulated at both the protein level and the gene level (Dwyer et al., 2007). At the protein level, a number of protein kinases and phosphatases are involved in controlling telomerase activity (Dwyer et al., 2007). For instance, the telomerase catalytic subunit hTERT is phosphorylated and activated by both PCK $\alpha$ and Akt in human breast cancer cells (Dwyer et al., 2007). Phosphorylation of an associated protein, TEP1, which binds to the RNA template has also been shown to increase telomerase activity. On the contrary, telomerase activity can be greatly reduced by the action of the protein phosphatase PP2A in breast cancer cells (Liu, 1999). The 
importance of the reciprocal action of $\mathrm{PKC} \alpha$ and $\mathrm{PP} 2 \mathrm{~A}$ in regulating telomerase activity is underscored by the fact that there is usually an imbalance in the action of these enzymes in cancer cells. The PKC $\alpha$ activity tends to exceed that of PP2A in breast cancer cells because of stimulation by an assortment of tumorigenic compounds and growth factors such as IGF1. The PP2A activity is likewise inhibited by the combined action of growth factors such as EGF and other tumorigenic antigens and compounds (Liu, 1999). The drug Tamoxifen is effective against breast cancer because it inhibits PKC $\alpha$ (Liu, 1999). The regulatory imbalance between PKC $\alpha$ and PP2A is significant because it results in elevated telomerase activity which in turn promotes tumorigenesis in breast cancer cells. It has even been suggested that the p53 protein may interact directly with telomerase to reduce its activity and thus may represent one mechanism by which the p53 gene acts as a tumor suppressor (Liu, 1999).

Besides regulation of telomerase activity at the protein level via the action of protein kinases and phosphatases, telomerase activity can also be regulated at the gene level- that is, at the level of gene expression or transcription. When discussing transcriptional control of telomerase expression, it is necessary to examine the hTERT promoter. It is well established that the c-Myc transcription factor is strongly linked to cellular proliferation, and it comes as no surprise that telomerase expression is upregulated by c-Myc. The c-Myc is able to interact directly with the hTERT promoter and stimulate hTERT expression. Transcription factors such as c-Myc stimulate gene expression by binding to specific sequences within the promoter region of a gene, and many cancer cells often accumulate mutations within the promoters of key cell cycle regulatory genes. 
In a large scale study of 799 tumor samples, Huang et al. (2015) identified two specific mutations which were present in the hTERT promoter in a high percentage of the tumor samples. Specifically, these mutations C228T and C250T occur at 124 and 146 bp upstream of the hTERT translation start site and are prevalent in many different types of tumors (Huang et al., 2015). The authors conclude that each mutation creates a new binding site for the E-twenty six (Ets) group of transcription factors which subsequently results in the upregulation of telomerase expression. Upregulation of telomerase activity has also been reported as a consequence of the hypoxia commonly found in solid tumors, where the enzyme may aid in the stabilization of chromosomal damaged induced by the low oxygen environment (Seimiya et al., 1999). Increased telomerase expression has also been observed in hepatocytes undergoing cell division in response to partial removal of liver tissue, and this telomerase expression is stimulated by both EGF and hepatocyte growth factor (HGF) (Inui et al., 2002). In each instance, the upregulation of telomerase expression was a direct response to signaling through the MAPK pathway.

The central role that the MAPK signaling pathway plays in telomerase activation is illustrated in a study by Maida et al. (2002). The effect of EGF stimulation on telomerase expression was investigated by exposing A-431, ME180, MCF-7, and NIH3T3 cells to EGF for various periods of time, and then assessing hTERT mRNA expression. There was a significant increase in hTERT mRNA expression in all of the cell types between 6 to 12 hours after EGF exposure. The increase in hTERT mRNA expression in response to EGF stimulation was confined to these cell types which constitutively express telomerase and was not observed in telomerase negative cell types. The authors conclude the MAPK pathway is primarily responsible for EGF induced telomerase expression. The A-431 cells 
stimulated with different concentrations of EGF for either 15 or 30 minutes showed a marked increase in ERK activity and hTERT expression. Exposure to the MEK inhibitor U0126 abolished this effect but exposure to PI3K or p38 inhibitors did not. Furthermore, the authors demonstrate that EGF exerts its influence on telomerase expression through the MAPK pathway via activation of the Ets family of transcription factors. The Ets group of transcription factors are phosphorylated and activated by ERK, and the hTERT promoter contains two Ets binding motifs within it at -23 and -18 . A-431 cells co-transfected with a wild-type Ets2 expression vector and an hTERT promoter reporter plasmid revealed high levels of EGF induced transactivation when compared to cells co-transfected with a truncated, dominant negative Ets2 expression plasmid.

A similar result was observed in cells transfected with either a normal hTERT promoter reporter plasmid or a plasmid containing mutations in the Ets binding motifs (Maida et al., 2002). The MPAK pathway has also been implicated in the activation of telomerase activity and hTERT mRNA expression in estrogen receptor $\alpha(E R \alpha)$ positive endometrial cells. Zhou et al. (2013) report that stimulation of Ishikawa ER $\alpha$ positive endometrial cells with estradiol (E2) results in increased phosphorylation p44/42 MAPK and increased hTERT mRNA expression. The hTERT promoter contains two binding sites for E2-ER $\alpha$ complexes, and the stimulatory effect of E2 on hTERT expression and telomerase activation was greatly reduced by exposure to the MEK inhibitor U0126 or ERK specific siRNA (Zhou et al., 2013). The MAPK pathway therefore appears to play a prominent role in the estrogen induced regulation of hTERT expression and telomerase activity.

As downstream targets of the MAPK pathway, the Ets family of transcription factors have recently been implicated in the regulation of telomerase gene expression and activity. 
The Ets family of transcription factors consists of more than thirty closely related proteins first identified in the E26 avain leukemia retrovirus. The Ets transcription factors display a conserved 85 amino acid helix-turn-helix motif capable of binding to a core consensus sequence (GGAA/T) located within the promoters of various target genes, which often times encode key proteins involved in the regulation of the cell cycle (de Launoit et al., 2006). The Ets group of transcription factors therefore have figured prominently into cellular proliferation and tumorigenesis. For instance, a number of studies have indicated that Ets1 and Ets2 are commonly overexpressed in a variety of cancers (de Launoit et al., 2006). The Ets2 transcription factor in particular is frequently upregulated in breast cancer cells. Dweyer et al. (2007) report that siRNA mediated silencing of Ets2 gene expression significantly reduced telomerase gene expression and activity in MCF7 breast cancer cells. There was also an interesting inhibition of c-Myc expression which suggests a potential role of Ets2 as a transcriptional activator of the c-Myc gene, which is a known transcription factor in the expression of telomerase. In this model, phosphorylation and activation of Ets2 by ERK results in enhanced transcription of c-Myc which in turn stimulates telomerase gene expression.

Telomerase expression is simultaneously enhanced by the binding of activated Ets 2 to the telomerase promoter and it is possible that Ets2 may even form a complex with c-Myc (Xu et al., 2008). In colon cancer, the formation of the Ets2/c-Myc complex may be mediated by Ruv-B like helicase 2 (Ruvbl2) which can interact with Ets2 (Flavin et al., 2011). Similarly, Hepatitis B virus core protein has also been shown to upregulate telomerase expression in conjunction with the Ets2 transcription factor in HepG2 cells (Gai et al., 2013). The specificity of Ets 2 for its binding sites underlies its ability to regulate 
telomerase expression. Xiao et al. (2003) investigated whether the binding of Ets2 to different Ets binding elements within the telomerase promoter had any influence on telomerase promoter activity. Mutagenic experiments revealed that specific Ets binding elements were associated with increased promoter activity while others were associated with decreased activity. Along the same line, Hsu et al. (2006) report that a single nucleotide polymorphism (SNP) located at -245 bp upstream of the telomerase promoter may act to increase telomerase activity in non-small cell lung cancer.

The importance of the Ets family of transcription factors in the regulation and expression of telomerase continues to be supported by a growing body of evidence. Goueli and Janknecht (2004) report that the Ets transcription factor ER81 is primarily responsible for upregulation of telomerase expression in SKBR3 breast cancer cells which overexpress the HER2/Neu receptor tyrosine kinase. The HER2/Neu receptor is overexpressed in the majority of breast tumors and its expression is likely enhanced by ER81. The authors also report that ER81 is capable of stimulating telomerase expression as it is a downstream target of the MAPK pathway. Active HER2/Neu can stimulate Ras which in turn will activate RAF1 and result in the downstream phosphorylation of ER81 by ERK. Once activated by ERK, ER81 can then stimulate expression of telomerase. Mutation of the ER81 binding sites within the telomerase promoter or inhibition of ER81 phosphorylation by ERK resulted in decreased telomerase expression and activity. A similar result was obtained by inhibition of HER2/Neu or the expression of a dominant negative form of ER81 (Goueli and Janknecht, 2004). The ER81, also known as Etv1, belongs to the PEA3 group of Ets transcription factors. The PEA3 transcription factors are known to regulate 
the expression of several matrix metalloproteases and may therefore figure prominently in the development of metastasis (de Launoit et al., 2006).

As mentioned previously, the overexpression of telomerase in most types of cancer cells is usually the result of aberrant cell signaling pathways. Much research has focused on identifying the principal molecules involved in controlling the expression of the telomerase promoter. The goal of promoter research is to be able to target expression of the enzyme at the transcriptional level. For example, in a study of 327 patients with urothelial cell carcinoma of the bladder, Rachakonda et al. (2013) described the existence of common mutations within the telomerase promoter. Mutations at 124 and $146 \mathrm{bp}$ upstream of the transcription start site were present in $65 \%$ of the samples. The mutations create new binding sites for the Ets/TCF group of transcription factors which increase promoter expression between two and four fold (Rachakonda et al., 2013). The presence of an additional single nucleotide polymorphism in an existing Ets binding site of the telomerase promoter appears to moderate the effect of these two upstream mutations. Patients without a variant allele of the SNP but with the two upstream mutations showed reduced survival and greater tumor recurrence when compared to patients with a variant allele of the SNP (Rachakonda et al., 2013).

Zhao et al. (2009) report that chromosomal mutations which translocate the human telomerase gene and its promoter (located near the telomere of chromosome $5 \mathrm{arm}$ p) to heterologous sites often results in transcriptional activation of the telomerase gene. The gene is not normally expressed in most somatic cells due to its location within highly condensed chromatin. However, translocation to other chromosomal sites with less 
restrictive chromatin structure may allow the telomerase promoter to become activated (Zhao et al., 2009).

The Wnt/beta-catenin signaling pathway has been shown to be involved in the activation of telomerase expression in a number of different types of human cancers. The Wnt pathway is crucial for normal cell differentiation and proliferation during embryonic development and beta-catenin is an effector of this pathway which can help activate the expression of Wnt target genes (Zhang et al., 2012). The Wnt/beta-catenin pathway is often active and not properly regulated in most cancer cells. When Wnt signaling is inactive, beta-catenin is targeted for destruction by phosphorylation. On the other hand, when Wnt signaling is active, beta-catenin is not targeted for destruction and travels into the nucleus where it binds to LEF/TCF transcription factors. The LEF/TCF transcription factors normally bind to DNA and repress the expression of target genes (Zhang et al., 2012).

Beta-catenin forms a complex with TCF4 and results in the expression of many genes (c-Myc, cyclin D1, and NOS2) which may contribute to cancer formation (Zhang et al., 2012). Zhang et al. (2012) provide evidence that the Wnt/beta-catenin pathway can also result in the activation of telomerase expression in human cancers. Using different cancer cell lines, the investigators were able to demonstrate that removal of beta-catenin by RNAi significantly reduced telomerase gene expression and activity in Wnt+ cancer cells. FH535, a beta-catenin/TCF inhibitor, was also shown to greatly reduce telomerase activity in all of the cancer cell lines studied (Zhang et al., 2012).

In a similar study of human colorectal carcinoma, Jaitner et al. (2012) were also able to demonstrate the ability of beta-catenin to regulate expression of the telomerase gene. The researchers determined that telomerase gene expression depends in part on the binding of 
the oncogene transcription factor c-Myc to the telomerase promoter, and that $\mathrm{c}-\mathrm{Myc}$ expression itself is stimulated by beta-catenin in Wnt signaling cells (Jaitner et al., 2012). However, a more interesting discovery was made in that the telomerase enhancer/promoter was determined to have four binding sites for beta-catenin/TCF- 4 complexes. Thus, it was shown that the telomerase gene can also be directly regulated by beta-catenin and in a way that is independent of c-Myc.

For instance, the data suggest that the expression patterns of telomerase and beta-catenin mirror one another but not that of c-Myc. c-Myc, while able to stimulate telomerase expression, is present ubiquitously in cancer cells and its expression appears to be controlled by various pathways (Jaitner et al., 2012). Zhang et al. (2012) suggest that in normal, non-cancerous cells c-Myc induced expression of telomerase is mediated by the suppressive effect of the E2F1 transcription factor. c-Myc promotes expression of E2F1 which then binds to the telomerase promoter and reduces the ability of c-Myc to activate expression of telomerase. The c-Myc and E2F1 stimulate each other's expression and this positive feedback mechanism is regulated in normal cells through the use of miRNAs (Zhang et al., 2012). Listerman et al. (2014) looked at whether or not telomerase itself can bind to and activate expression of Wnt target genes. The HeLa cells and human breast cancer cell lines were used in the study and the authors failed to find any evidence for the ability of telomerase to interact directly with beta-catenin or the chromatin remodeling protein BRG1. Additionally, altering the levels of telomerase in the breast cancer cell lines had only a minimal effect on Wnt signaling and target gene expression (Listerman et al., 2014). 
The Hedgehog signaling pathway, which is important in cell specialization and tissue formation during embryonic development, is abnormal in a number of different types of cancer cells (Mazumdar et al., 2013). The Hedgehog pathway results in the activation of the GLI group of transcription factors. Mazumdar et al. (2013) investigated whether telomerase is a target of Hedgehog signaling via GLI transcription factors. Inhibition of GLI1 and GLI2 through various mechanisms resulted in a significant decrease in telomerase expression in prostate cancer, Glioblastoma multiforme, and colon cancer cell lines compared to controls. GLI1 and GLI2 were shown to interact directly with the telomerase promoter by chromatin immunoprecipitation (Mazumdar et al., 2013). The results indicate that telomerase expression can be influenced by abnormal Hedgehog signaling.

The role that the deacetylase enzyme SIRT1 plays in transcriptional activation of the telomerase promoter in hepatocellular carcinoma was investigated by Zhang et al. (2014). Epigenetic modifications of the chromatin structure of promoter and enhancer regions of DNA is a primary mechanism by which cells regulate activation of transcription and thus gene expression. Acetylation of histones promotes transcription by relaxing the association of DNA with histone proteins while methylation of histones appears to reduce transcription by increasing chromatin condensation. The researchers hypothesized that SIRT1 plays an important role in remodeling the chromatin of the telomerase promoter which then results in enhanced expression of telomerase in hepatocellular carcinoma. The results indicate that SIRT1 does not regulate telomerase expression via $\mathrm{CpG}$ island methylation at the telomerase promoter (Zhang et al., 2014). Additionally, the data suggest that the inhibition of SIRT1 in hepatocellular carcinoma SK-HEP-1 cells results in acetylation of the ninth 
lysine residue of histone $\mathrm{H} 3$ as well as reduced methylation at the same position (Zhang et al., 2014). These epigenetic changes to the chromatin structure of the telomerase promoter should increase telomerase expression. However, the researchers observed the opposite. Telomerase expression was reduced and the authors offer no explanation for the mechanism of action.

Yamada et al. (2012) studied the ability of Interleukin-2 (IL-2) to stimulate telomerase expression in adult $\mathrm{T}$ cell leukemia (ATL) cells. The results show that IL-2 binds to its receptor in the cell membrane and consequently stimulates a number of signaling pathways that ultimately lead to telomerase expression. The binding of IL-2 results in phosphorylation of tyrosine residues in Janus activated kinases 1-3. Activated JAK1 and JAK2 then phosphorylate the transcription factor STAT5 which travels into the nucleus and binds to the telomerase promoter, resulting in the expression of telomerase (Yamada et al., 2012). At the same time, activated JAK2 can stimulate the PI3K/AKT/mTORC1/S6K pathway in ATL cells as well. The pathway increases telomerase expression both transcriptionally and post-translationally. Knockdown of STAT5 by RNAi was also shown to reduce telomerase expression (Yamada et al., 2012). Human lymphoblastoid cells were used as a positive control for the telomerase activity assays and beta actin was used as a positive control for telomerase mRNA assessment by RT-PCR.

Prostate cancer provides an excellent example of the influence of androgen signaling pathways on telomerase expression. Liu et al. (2010) examined LNCaP prostate cancer cells with the goal of determining how androgen signaling affects expression of telomerase. A dose dependent response in telomerase promoter activity with various concentrations of 
DHT was observed. Higher concentrations resulted in greater activity. Inhibition of androgen receptor expression by RNAi resulted in a significant reduction of telomerase mRNA in LNCaP cells. The investigators were also able to demonstrate that the drug methylseleninic acid (MSA) can also reduce telomerase mRNA levels by inhibiting the synthesis of androgen receptor (AR). The fact that androgen activated AR can bind to the telomerase promoter and facilitate transcription was confirmed by chromatin immunoprecipitation assay.

Holysz et al. (2013) highlight the significant role that telomerase has in the etiology of breast cancer. The authors cite numerous studies which indicate that transcriptional activation of the telomerase gene promoter by various transcription factors such as c-Myc or wnt appears to be a key step in the transformation of normal cells into malignant cancer cells (Bernardes de Jesus \& Blasco, 2013). The telomerase promoter has several binding sites for transcription factors which are involved in a number of cellular pathways. For instance, production of transcription factors induced by the binding of human epidermal growth factor 2 (Her2) as well as the transcription factor ER81 have both been shown to cause increased expression of telomerase in human breast tissue (Holysz et al., 2013). Estrogen has been demonstrated to cause a similar effect by binding to and activating a cytoplasmic receptor which then travels to the nucleus and can activate control elements linked to the telomerase promoter.

If the telomerase promoter can be activated by various transcription factors which are normally present in cells, why then is telomerase not expressed continuously in most somatic cells? The answer seems to lie in the action of proteins encoded by tumor suppressor genes. In breast tissue, the protein product of the BRCA1 tumor suppressor gene 
interacts with c-Myc and prevents it from binding to regulatory regions of the telomerase promoter. The BRCA1 gene product has also be shown to interfere with the proteins TRF1 and TRF2 which are necessary to recruit telomerase to the telomere regions of chromosomes (Holysz et al., 2013). The protein product of the p53 tumor suppressor gene acts in a similar fashion by interacting with certain transcription factors which could potentially activate the telomerase promoter. The result of the interaction is decreased expression of the telomerase gene (Bernardes de Jesus \& Blasco, 2013).

Telomerase is active and overexpressed in a number of other cancers besides breast cancer. Small cell lung cancer as well as non-small cell lung cancer both exhibit high levels of telomerase activity, with SCLC being telomerase positive in approximately $90 \%$ to $100 \%$ of cases (Chen \& Chen, 2011). High levels of the enzyme is correlated with poor survival in both types of lung cancer. The role that telomerase plays in gastric cancer is less certain. A few studies have indicated that a high level of telomerase activity is associated with the degree of malignancy of a tumor (differentiation and depth of invasion) while other studies have been unable to arrive at such a conclusion (Chen \& Chen, 2011). However, what has been demonstrated more clearly is that the presence of telomerase in the peritoneal fluid of gastric cancer patients is a clinical sign of advanced stages of the disease, including metastasis (Chen \& Chen, 2011).

The exact role that telomerase plays in renal and intestinal cancers is uncertain. The tissues of these organs normally express detectable levels of the enzyme, and it is often difficult to establish a threshold value of telomerase activity which is associated with malignancy. Once an adenomatous polyp forms in the colon, telomerase activity has shown some relationship to the grade and size of the polyp. However, in most colon cancers it 
seems that the presence of high levels of telomerase mRNA is a good indicator of metastasis. Telomerase activity in renal cancer has not been shown to be correlated with any clinical parameters or survival (Chen \& Chen, 2011). Although about $90 \%$ of all bladder cancers display high levels of telomerase activity, it is not always associated with malignancy. Normal bladder tissue can test positive for the enzyme, which again, makes it difficult to distinguish the fine line between normal and abnormal (cancerous) levels of activity. Most uterine cancers fall under the same umbrella because of the fact that endometrial cells express varying levels of telomerase activity normally depending on the stage of the menstrual cycle (Chen \& Chen, 2011).

Finally, telomerase levels and activity appear to mirror well the transition that cervical cells undergo as they become cancerous. As cervical cells progress through the three nonmalignant stages prior to becoming malignant, there appears to be an increasing trend in telomerase expression and activity. An estimated $90 \%$ of cervical cell carcinomas will eventually display elevated levels of telomerase mRNA and activity (Chen \& Chen, 2011). In ovarian cancer, telomerase expression and activity have been shown to vary widely depending on the clinical classification of the tumor (Chen \& Chen, 2011). In comparison to most carcinomas, sarcomas typically display reduced levels of telomerase activity and seem to rely on other non-telomerase mediated mechanisms to lengthen their telomeres (alternative lengthening of telomeres or ALT) (Chen \& Chen, 2011). For example, Hu et al. (2013) demonstrated in transgenic mice that alternative lengthening of telomeres mechanisms linked to mitochondrial functions can even be activated in lymphomas as well. The transgenic mice contained an inducible telomerase expression system which could be manipulated by the researchers. Inhibition of telomerase expression in the lymphoma cells 
resulted in an initial decrease in the growth of the cancer cells but also resulted in the subsequent activation of alternative lengthening of telomeres pathways and the survival of the tumors.

\subsection{Telomerase as a Cancer Target}

Telomerase inhibition in cancer cells has been active area of research because, as mentioned earlier, a large percentage of different cancers express telomerase at high levels compared to normal, healthy cells. This important difference offers the promise of developing therapies that are specific to cancer cells while helping to minimize the adverse side effects observed with many conventional therapies. There are three broad strategies currently available to target telomerase. The first of which is the use of drugs, or medications, which interfere with the biochemical activity of the enzyme. A classic example is the drug GRN163L (Imetelstat). Imetelstat works by preventing the binding of the RNA template component of telomerase with the protein portion of the enzyme. The end result of this interaction is a nonfunctional enzyme. However, there is a lag period of time after initial exposure of cancer cells to a telomerase inhibitor and reduced tumor growth. This lag period is partially a result of the time it takes for telomeres to become shortened enough in the presence of the drug to allow for activation of senescence or apoptosis (Buseman et al., 2012). Often times, it is recommended to combine treatment with a telomerase inhibitor with traditional therapies such as radiation and chemotherapy. The combination therapy could prevent any further growth of the tumor during this lag phase of time before the drug starts to have an effect. Telomerase inhibitors have also been

proposed as maintenance drugs following conventional treatment as a way of possibly 
inhibiting any residual cancer cells from causing a relapse of the disease (Buseman et al., 2012).

The second category of telomerase inhibition is immunotherapy, or vaccination. Many telomerase positive cancer cells express telomerase specific antigens, or peptides, on their cell membranes. Vaccines can be developed from protein fragments of the telomerase enzyme or from actual AP (antigen presenting) cells which display telomerase peptides on their surfaces. A few vaccines of these types are currently in clinical trials and most show promising results (Buseman et al., 2012).

The third type of telomerase inhibitor makes use of gene therapy. The telomerase gene promoter has been linked to apoptotic genes such as TRAIL or a caspase. Through a viral mechanism or some other gene delivery format, these apoptosis inducing genes under the control of the telomerase promoter can be introduced into cancer cells. The cells will then express these genes whenever the telomerase promoter is transcribed. Alternatively, adenoviruses have been engineered in which two viral genes necessary for replication only are under the control of the telomerase promoter. Viral infection of cancer cells results in the expression of the telomerase promoter and virus genes, resulting in the lysis of the cancer cells. A clinical trial with Telomelysin, an engineered adenovirus, is currently underway and the preliminary results appear encouraging despite notable failures of this approach in the past (Buseman et al., 2012).

The important role that telomerase plays in the life of most cancer cells is illustrated by the number of research articles which have been published within the past five to six years investigating various aspects of this enzyme with respect to cancer cell survival or mortality. These articles can be broadly grouped according to their design as investigating 
the effects of drug inhibitors, immunotherapy/gene therapy, and RNAi on the structure and/or function of telomerase.

Liu et al. (2012) investigated the effect of the synthetic compound CDDO-Me, an oleanane triterpenoid, on telomerase activity in mice prostate cancer cells. The researchers discovered that this drug exerts its anti-tumorigenic effects in two main ways. First, it interferes with the synthesis of transcription factors such as c-Myc, SP1, and NF-kB which are necessary to initiate transcription of the telomerase promoter. The phosphorylation status of another necessary transcription factor, STAT3, is interfered with by the drug as well (Liu et al., 2012). Secondly, the telomerase enzyme must be phosphorylated on serine residues 227 and 826 to become activated. The CDDO-Me drug prevents the enzyme Akt from performing this function (Liu et al., 2012). The Sp family of transcription factors, which are typically found at high levels in many cancer cells, are an ideal target of the drug. The SP1 transcription factor interacts with c-Myc to stimulate the production of telomerase (Liu et al., 2012). The inhibition of telomerase by CDDO-Me via transcriptional and posttranslational mechanisms more than likely allows for the induction of apoptosis in prostate cancer cells. Moreover, there appears to be a dose-dependent response with levels of the drug in the 1.25 to $5 \mathrm{uM}$ range producing a significant reduction in tumor growth (Liu et al., 2012). Deeb et al. (2012) investigated the potential use of CDDO-Me in pancreatic cancer cells and reported similar results to those of Liu et al. Proliferation of cells was arrested and apoptosis was induced. Interestingly, overexpression of telomerase by MiaPaCa-2 and Panc-1 cancer cells transfected with a telomerase expression plasmid resulted in these cells being significantly less sensitive to concentrations of the drug than dosages that would otherwise kill other cancer cells (Deeb et al., 2012). 
The structure of telomeric DNA is complex, with a DNA loop that gives rise to guanine rich tetra-stranded structures called G-quadruplexes. The G-quadruplex structures found at telomeres tend to inhibit telomerase function by preventing the attachment of the RNA template of the enzyme to DNA in these regions (Huang et al., 2012). The ability to stabilize these regions of telomeric DNA could therefore be very useful in reducing telomerase activity in cancer cells. The compound, BMVC4, has been shown to do just this. Huang et al. (2012) treated human lung cancer cells, H1299, with various concentrations of BMVC4 and noted the expected onset of senescence. Cells which overexpressed telomerase as well as cells which lengthened telomeres by an alternative mechanism were tested. The fact that senescence was observed in both cell types indicates that telomerase inhibition may not be the sole cause by which BMCV4 operates. The investigators worked out that in addition to stabilizing telomeric G-quadruplexes, the drug also stabilizes a G-quadruplex structure in the promoter of the c-Myc gene (Huang et al., 2012). The drug-promoter interaction prevents the production of the c-Myc transcription factor that plays a role in telomerase gene expression. Senescence in both cell types may also have been triggered by the activation of the ATM kinase DNA damage pathway in response to DNA breaks caused by exposure to the drug (Huang et al., 2012).

In a very similar fashion, Yu et al. (2012) investigated the ability of ruthenium (II) polypyridyl complexes to stabilize G-quadruplex DNA. Two different enantiomers were tested, alpha and delta, and it was determined that the alpha form had the greatest ability to bind to G-quadruplex DNA and stabilize it in HepG2 cancer cells (Yu et al., 2012). This stabilization was confirmed through a series of chemical analyses as well as TRAP (telomeric repeat amplification protocol) assay. In HeLa cells however, the delta 
enantiomer had a greater cytotoxic effect. In fact, the degree of cytotoxicity was shown to depend largely on the specific type of cancer cell tested. Although the study employed a wide range of chemical tests, the results are mostly qualitative and the authors do not identify the precise mechanism of ruthenium complex binding to G-quadruplex DNA (Yu et al., 2012).

In a 2010 study, Marian et al. evaluated the efficacy of the drug Imetelstat (GRN163L) on the ability to inhibit telomerase in glioblastoma tumor initiating cells. Glioblastomas are often fatal and patients usually have only a few months to live once diagnosed. The two year survival rate is less than five percent (Marian et al., 2010). As discussed earlier, Imetelstat targets the RNA component of the telomerase enzyme and prevents the formation of functional telomerase. The drug is particularly well suited to permeating tissues because of a lipid component in its chemical structure that is able to cross the bloodbrain barrier (Marian et al., 2010). The authors report that the anti-tumorigenic effects of Imetelstat begin to occur after about 15 to 20 rounds of cell division after exposure to the drug, with $\mathrm{IC}_{50}$ telomerase inhibition occurring at a concentration of $0.45 \mathrm{uM}$ (Marian et al., 2010). Unfortunately, normal healthy populations of brain stem cells are also affected by the drug. However, as a consequence of a key difference in the actual length of telomeres between glioblastoma cells and normal stem cells, the drug has less of a negative effect on the normal stem cells. The negative effect is reversed when treatment is stopped. The researchers also acknowledge the fact that most of the observed results and data of Imetelstat treatment were collected from mice subcutaneous xenografts of glioblastoma tumor cells (Marian et al., 2010). 
Beisner et al. (2009) report limited success in inhibiting telomerase activity with use of antisense 2'-O- methyl-RNA. The RNA molecule was packaged into chitosan coated PLGA nanoparticles which were readily absorbed by A549 cancerous lung cells. The RNA molecule acts in a similar way to the drug Imetelstat in that it interferes with the RNA template of the telomerase enzyme. However, the anti-tumorigenic effects of the molecule as measured by a TRAP assay are not observed until after approximately fifteen weeks of treatment (Beisner et al., 2009). An appropriate experimental design which includes a series of controls for the delivery of the nanoparticles and for the specificity of the RNA molecule for the telomerase RNA component is apparent. A549 cells treated with 2'-Omethyl-RNA saw an average decrease in telomere length of about 2,000 bp when compared to control groups (Beisner et al., 2009).

Mohammad et al. (2013) looked at the therapeutic effect of a plant compound called Diosgenin derived from the herb Trigonellafoenum graceum. The authors mention a number of studies which have already demonstrated the telomerase inhibiting ability of this compound, and their work seems to be mostly a repeat and confirmation of previous studies. A549 lung cancer cells were treated with various concentrations of Diosgenin and the ability to inhibit telomerase activity displayed a dose dependent and time dependent function. Telomerase activity was determined by qRT-PCR using telomerase mRNA extracted from Diosgenin treated cells (Mohammad et al., 2013). The results clearly indicated reduced levels of telomerase mRNA in treated cells but no statistical analysis is provided nor do the authors discuss any observed reduction in A549 cell growth. The authors do not mention an exact mechanism for how Diosgenin could down-regulate 
telomerase gene expression other than to suggest that the drug most likely down-regulates expression of c-Myc (Mohammad et al., 2013).

Chen et al. (2013) report that epigallocatechin gallate (ECGC) found in green tea and sulphoraphane (SFN) found in vegetables such as broccoli can produce anti-tumorigenic effects in ovarian cancer cells when used in combination. The researchers primarily wished to test the inhibitory of effects of these compounds, either alone or in combination, on cancer cells which were resistant to the chemotherapy drug Paclitaxel. The design of the study seems fairly straightforward, but there is no real effort to explain which controls were used when treating two cells lines of ovarian cancer with various combinations of ECGC and/or SFN. A paclitaxel susceptible cell line (SKOV3-ip1) was compared to a resistant cell line (SKOV3TR-ip2). The authors state that the resistant cell line trials were exposed to Paclitaxel in their growth media, but no control was made to expose the sensitive cells to the drug to indeed show that they are susceptible. Although each cell type was tested according to ECGC alone, SFN alone, and ECGC + SFN and for the same periods of time (24, 48, and 72 hours), the concentrations of ECGC alone and SFN alone were different in each trial (Chen et al., 2013).

A possible negative control of either resistant or susceptible cells exposed to DMSO only was included as a reference in each trial at each time interval. However, how a statistical analysis comparing cell growth in drug conditions as a percent of DMSO control cell growth is not clearly indicated. The investigators also assayed each cell line for the presence of the anti-apoptotic protein Bcl-2 and for telomerase activity. The results suggest that the expression of both proteins as well as DNA methyltransferase-1 (a chromatin remodeling enzyme) are significantly reduced in each type of cell line when used in 
combination (Chen et al., 2013). A beta actin positive protein control was used as a comparison for the Western blot analysis. Overall, the authors conclude the SFN alone can significantly reduce the growth of cells in both Paclitaxel resistant and sensitive cell lines. When used in combination with ECGC, the result is amplified; however, no substantial detail on the mechanism of action for how these drugs affect cell growth or protein expression is provided (Chen et al., 2013).

In an almost identical design to that of Chen et al., Nasiri et al. (2013) treated T47D human breast cancer cells with the herbal compounds curcumin and/or silibinin. Not surprisingly, the results indicated that a combination of the two plant compounds at high concentrations for long periods of time had a detrimental effect on the viability of T47D cells as measured by MTT assay (Nasiri et al., 2013). Quantitative real time PCR was employed to assess telomerase expression as a function of treatment with curcumin and/or silibinin. Again, the controls were not clearly defined for either part of the experiment with only the mention that beta actin served as an endogenous control for the comparison of telomerase expression (Nasiri et al., 2013).

While not an actual drug, the glycolytic enzyme glyceraldehyde 3-phosphate dehydrogenase has been shown to be able to induce apoptosis in cells subjected to oxidative stress. The enzyme is involved in limiting the formation of reactive oxygen species in addition to its central role in glycolysis. Nicholls et al. (2012) provide evidence that GAPDH can travel into the nucleus in response to oxidative stress and can interact with telomeric DNA. This interaction normally stabilizes the telomeres and promotes cell survival. The enzyme has also been shown to interact with the RNA template of telomerase 
and thus inhibits the action of telomerase. Nicholls et al. (2012) observed increased senescence in MCF7 breast cancer cells stably expressing a recombinant GAPDH.

Another intriguing approach to investigate the role of telomerase in cancer cells is through the use of immunotherapy/gene therapy. These techniques make use of the fact that many cancer cells begin to express altered peptides on their cell membranes as a result of accumulated genetic and chromosomal abnormalities. The cells of the immune system, cytotoxic T cells in particular, are able to identify cancer cells as foreign because of their surface molecules and can then target them for destruction. Miyazaki et al. (2013) were able to kill adult $\mathrm{T}$ cell leukemia (ATL) cells using modified CD8+ T cells. The CD8+ T cells were transfected with a retroviral expression vector containing genes which would enable these modified CD8+ T cells to identify a very specific protein complex found on the surface of ATL cells over-expressing telomerase. That complex is HLAA*24:02/hTERT $461-469$. Mice were injected with ATL cells containing this complex. The mice were then injected with either modified CD8+ T cells, unmodified CD8+ T cells, or no cells at all. Compared to the control groups, the mice which received the modified CD8+ T cells showed minimal levels of ATL cell growth over a six month period (Miyazaki et al., 2013).

The ability to produce a sustained response by $\mathrm{CD} 8+\mathrm{T}$ cells against telomerase expressing tumor cells requires the assistance of CD4+ helper T cells. The helper cells play a role in maintaining cytotoxic $\mathrm{T}$ cell numbers and also play a critical role in generating memory immune responses. Liao et al. (2013) examined the effect of dendritic cell stimulation with branched diepitope multiple antigen peptides (MAPS) on the ability to lyse various different tumor cell lines expressing telomerase. The particular MAP used 
contained two epitopes of human telomase, one which would stimulate CD8+ cytotoxic T lymphocytes and another which would stimulate CD4+ helper T cells. The diepitope MAP resulted in a slight increase $(8.56 \%)$ in the ability of cytotoxic T lymphocytes to lyse target tumor cells than when cytotoxic T lymphocytes were exposed to just the CD8+ epitope of human telomerase alone (Liao et al., 2013). A positive control of dendritic cells exposed to a recombinant adenovirus human telomerase vector and a negative control of dendritic cells exposed to an HIV peptides were used when comparing the ability of branched monoepitope MAPs to cause CTL induced lysis of target cells versus their linear forms. As already mentioned, a diepitope MAP produced the greatest immune response (Liao et al., 2013).

Adotevi et al. (2010) researched a lentivector model for inducing the proliferation of endogenous anti-telomerase CD8+ $\mathrm{T}$ cells in mice. The HLA-A*0201 transgenic HHD mice were injected with a lentiviral expression system containing the human telomerase gene. Mouse cells expressing an epitope of human telomerase on their surfaces then induced the development of CD8+ T cells capable of detecting the human epitope. Surprisingly, these cells also displayed a strong ability to target self (mouse) cells which expressed epitopes derived from mouse telomerase (Adotevi et al., 2010). When inoculated with B16/A2 mouse melanoma cells, mice which were immunized with the lentiviral vector resulted in tumor regression in three out of ten mice. Seven out of ten mice were cured of their tumors when a specific telomerase peptide epitope was injected along with the lentiviral vector. All mice in a control group not exposed to the lentiviral vector died forty days after tumor cell inoculation (Adotevi et al., 2010). 
Using a different lentivirus model, Yu et al. (2011) demonstrated the ability of a lentiviral vector consisting of the cytosine deaminase (CD) suicide gene and the green fluorescent protein (GFP) gene under the control of an optimized human telomerase gene promoter. It was hypothesized that infection of various cancer cell lines with the vector would result in transcription of the telomerase promoter and the expression of the CD gene, which could be visually confirmed by the simultaneous expression of GFP. Upon administration of the drug 5-FC, the cancer cells would convert it into 5-fluorouracil using the cytosine deaminase enzyme. This conversion in turn would result in the death of the cancer cells. Appropriate controls with telomerase negative cancer cells along with the use of a vector lacking the $\mathrm{CD}$ gene were utilized as part of the experiment. The results indicate that intra-tumor injection of the CD/GFP lentiviral vector results in a significant difference in tumor volume when compared to controls (Yu et al., 2011).

In recent years, the use of RNA interference (RNAi) pathways in cells has led to a number of important discoveries about gene expression and protein function. RNA interference makes it possible to study the silencing of specific genes and how cellular pathways may be affected by the reduced expression of a particular protein. The potential therapeutic benefits of RNAi to interfere with cancer cell proliferation have become the basis for a number of innovative cancer treatments. Cerone et al. (2011) exposed HeLa cells to an siRNA library which contained siRNAs directed against a number of different kinase enzymes involved in regulating telomerase activity. The authors found that RNAi of the protein kinases MAPKAPK5 and ERK8 resulted in a significant decrease in telomerase mRNA levels in HeLa cells when compared to cells transfected with siRNA controls not directed against protein kinases (Cerone et al., 2011). Silencing of the enzyme 
SRC through the siRNA screen resulted in a significant increase in telomerase mRNA, which suggests that the kinase normally has an inhibitory effect on telomerase expression. The RNA interference inhibition of kinases such as ERK8 resulted in telomere length reduction and DNA damage, which was detected by the presence of DNA damage proteins TRF1 and 53BP1 at the telomeres (Cerone et al., 2011). Telomerase activity was measured by RT-PCR using cells transfected with non-kinase directed siRNAs as a negative control. A positive control of cells transfected with siRNA directed against telomerase was also present.

The key role of tumor suppressor gene products such as p53 and p21 were discussed in an earlier section of the paper. One of the hallmarks of many different types of cancer cells is the mutational loss of these critical cell cycle control proteins. Yoon et al. (2011) investigated whether or not the microRNA miR-296 is able to regulate expression of p53 and/or p21. Using luciferase reporter plasmids which contained either the 3' UTR of p53 or $\mathrm{p} 21$, the researchers were able to demonstrate that miR-296 only weakly associates with p53 mRNA. However, there is a very strong binding of miR-296 to the 3' UTR of p21 (Yoon et al., 2011). As a consequence, this binding would result in a large downregulation of $\mathrm{p} 21$ protein levels and thus a loss of the important tumor suppressor role of this protein. The stability of p21 mRNA is enhanced by the binding of HU proteins to the 3' UTR. The miR-296 can bind to these HU sites instead and cause increased instability of the p21 mRNA (Yoon et al., 2011). The result was observed in a variety of different cancer cell lines.

The success of RNAi modalities to limit cancer cell growth depends largely on an appropriate delivery system of the RNAi agent. In a 2012 study, Xia et al. tested the ability 
of a modified polyethylenimine (PEI) delivery system to effectively deliver a siRNA directed against telomerase mRNA into hepatocelluar carcinoma HepG2 cells. The use of a cationic compound such as polyethylenimine does not elicit an immune response the way a viral delivery vector could, and has the ability of forming nanoscale particles with siRNA allowing for ease of transfection of target cells (Xia et al., 2012). The disadvantage of PEI is that it is highly cytotoxic as a result of low degradability. Xia et al. modified PEI by introducing a disulfide bond which would make it more easily degradable in cells and thus reduce its cytotoxicity. The researchers injected HepG2 tumor cells into four different groups of isogenic mice to induce tumor growth, with five mice in each group. Once a tumor had formed, one group of mice received injections of disulfide PEI/siRNA, one group received normal low molecular weight PEI/siRNA, another group received disulfide $\mathrm{PEI} /$ non-telomerase siRNA, and the fourth group remained untreated. A significant decrease in tumor volume over a seven day period was observed for the disulfide PEI/siRNA treated group compared to the untreated group. However, the reduction was fairly minimal and appeared to only slightly delay tumor growth (Xia et al., 2012). In a separate experiment, the authors were able to show that rats administered low doses of disulfide PEI (5 or $50 \mathrm{ug} / \mathrm{kg}$ ) displayed no adverse effects on liver or kidney function compared to rats administered PBS as a control (Xia et al., 2012).

Gandellini et al. (2007) report success of siRNA treatment of prostate cancer cells. Two androgen independent prostate cancer cell lines, PC-3 and DU145, were transfected with fourteen different siRNAs. Two siRNAs were shown to produce a large decrease in telomerase activity in these cell lines when compared to the same cell lines transfected with non-telomerase directed siRNA. Exposure to just the transfection agent alone also did not 
affect telomerase activity (Gandellini et al., 2007). To test whether the significant decrease in telomerase activity seen with the two particular siRNAs was actually a result of inhibition of telomerase mRNA translation and not some other mechanism, telomerase negative osteosarcoma cells were transfected with the siRNAs of interest. Osteosarcoma cell growth was not affected. The researchers were also able to demonstrate that the observed reduction in prostate cancer cell telomerase activity was not caused by activation of an interferon pathway as no increase in expression of an interferon response gene was observed in siRNA treated and control cells (Gandellini et al., 2007). Lund et al. (2008) investigated the effectiveness of a lentiviral vector system expressing a small hairpin RNA directed against a portion of the canine telomerase RNA template molecule. The authors state that, on the basis of their previous work, canine telomerase function and activity is more similar to human telomerase than is murine telomerase, and is thus a better model to study possible therapeutic effects in humans (Lund et al., 2008). Mice injected with canine hemangiosarcoma tumor cells were used in the study. The results suggest that RNAi directed against the canine telomerase RNA component is able to cause a rapid decrease in tumor volume and enhance apoptosis, but a population of tumor cells became resistant and began to grow through an alternative lengthening of telomeres mechanism (Lund et al., 2008). The side effect has been documented in other studies and underscores the ability of anti-telomerase therapies to possibly induce alternative lengthening of telomeres in certain types of cancer cells.

Lastly, Uziel et al. (2010) report that miRNA expression can change as a function of telomere length. The researchers screened for approximately 900 different miRNAs in SKN-MC cells which had normal length telomeres, shortened telomeres due to telomerase 
inhibition, and telomeres restored back to normal length with restoration of telomerase activity. The profile of miRNA expression changed in cells with shortened telomeres compared to the other two cell types. Most of the differentially expressed miRNAs were related to cell growth inhibition and/or apoptosis (Uziel et al., 2010). Micro RNAs could even be useful in interfering with the regulation of alternative splicing of the telomerase mRNA. Wong et al. (2013) have shown that the telomerase mRNA can be spliced in various ways, some of which result in nonfunctional enzyme. Indeed, the use of RNAi to prevent normal splicing of telomerase mRNA could represent a novel form of chemotherapy.

\subsection{The Role of Endocytosis in Cancer Cell Migration}

One of the defining hallmarks of most cancers, besides unregulated cell proliferation and telomerase activity, is cell migration. A growing body of research is beginning to shed light on the central role that defective endocytosis plays in promoting cancer cell migration. Endocytosis is instrumental in a number of different tumorigenic processes ranging from receptor tyrosine kinase internalization to regulating cell membrane dynamics. Recently, the Rab family of small GTPases have been implicated in cancer cells as an important cause of the altered endocytosis that may lead to enhanced cell migration. Rab5 is of particular interest given its primary function in regulating the early endosome fusion and trafficking events that are necessary for cancer cell migration.

A significant body of evidence suggests that Rab5 mediated endocytosis plays a crucial role in the cellular processes that govern cancer cell migration. Endocytosis is a normal cellular process that involves the uptake or internalization of extracellular material and cell membrane components through the formation of endosomes. There are four primary 
mechanisms by which endocytosis occurs: clathrin-mediated endocytosis, caveolaemediated endocytosis, clathrin and caveolin independent endocytosis, and macropinocytosis (Mosesson et al., 2008).

\subsection{A. Modes of Endocytosis}

Clathrin-mediated endocytosis is characterized by the uptake of activated plasma membrane receptors via the formation of clathrin coated pits- invaginations of the cell membrane covered in clathrin protein. The scission of clathrin coated vesicles (endosomes) from the membrane and subsequent endosome trafficking requires the action of numerous proteins such as the dynamin GTPases, AP2, and the Rab family of GTPases. Once internalized, these vesicles shed their clathrin coats and fuse with other tubule-vesicular compartments to form structures known as early endosomes that later give rise to a cargo sorting structure known as the multi-vesicular body (Mellman and Yarden, 2013). Rab proteins, in particular Rab5, are instrumental in the coordination of early endosome formation and fusion events as well as the sorting of internalized cellular cargo. The slightly acidic $\mathrm{pH}$ of early endosomes promotes the dissociation of ligands from their receptors (Mellman and Yarden, 2013). Internalized membrane receptors from the surface of the cell will be either routed to lysosomes for destruction or recycled back to the plasma membrane through the coordinated action of the endocytic machinery involving Rab proteins (Mosesson et al., 2008). Receptors destined for degradation in lysosomes are typically ubiquitinated (Mellman and Yarden, 2013).

Caveolin-mediated endocytosis occurs through a similar mechanism to that of clathrinmediated endocytosis with the exception of the formation caveolin protein containing, cholesterol rich lipid rafts from the cell membrane. These vesicles are then targeted to other 
caveolin containing organelles termed caveosomes (Mosesson et al., 2008). Clathrin and caveolin independent endocytosis is poorly understood and may involve the uptake of glycosylphosphatidylinositol- anchored proteins from the plasma membrane. Finally, macropinocytosis is characterized by the bulk internalization of receptor tyrosine kinases through the Rab5 dependent formation of circular dorsal ruffles (Mosesson et al., 2008).

\subsection{B. Rab5 and Endocytosis in Cancer Cell Migration}

The role that endocytosis plays in tumorigenesis is becoming clearer. Multiple studies have shed light on the ability of the endocytic machinery to regulate a wide variety of cellular processes including signal transduction from activated receptors, cell morphology, cell junctions, and cell migration/invasion. Indeed, many cancers often display abnormalities in one or more of these endocytosis mediated events, and the essential role of Rab proteins in determining endosome identity as well as facilitating endosome fusion and transport has been an intense area of cancer biology research (Mendoza et al., 2014).

The Rab (Ras associated in brain) family of proteins belong to the Ras superfamily of small GTPases and more than 60 different Rab proteins have currently been identified. Like the Ras GTPases, the Rab GTPases are monomeric cytosolic proteins that act as molecular switches by alternating between an inactive GDP bound state and an active GTP bound state. The dissociation of GDP and binding of GTP is regulated by guanine nucleotide exchange factor (GEF) proteins while the hydrolysis of GTP to GDP is regulated by GTPase activating (GAP) proteins (Mendoza et al., 2014). Rab5 is particularly important for the fusion of early endosomes and the routing of internalized cellular cargo from these endosomes. Rab5 has also been implicated in cell migration through the regulation of actin remodeling of the cytoskeleton following receptor tyrosine kinase 
activation by means of a mechanism involving integrin internalization and recycling (Subramani and Alahari, 2010).

Integrins are transmembrane glycoproteins that are comprised of heterodimers of $\alpha$ and $\beta$ subunits and are major components of specialized plasma membrane regions known as focal adhesions. Focal adhesions are characterized by collections of various integrins and adaptor proteins that serve to link the cell to the surrounding extracellular matrix (ECM) (Subramani and Alahari, 2010). Integrins such as $\alpha 5 \beta 1$ bind to fibronectin in the ECM and the $\beta 1$ integrin subunit is known to associate with activated Rab5 (Tang and Ng, 2009; Mendoza et al., 2014). The endocytosis mediated turnover of focal adhesions through the recycling of integrin complexes is a major driver of cancer cell migration (Mendoza et al., 2014). In this focal adhesion model, growth factor induced cell migration consists of four key steps: formation of circular dorsal ruffles and lamellipodia, dissolution of focal adhesions at the rear of the cell, formation of new focal adhesions at the leading edge of the cell, and the 3D movement of the cell to a different location (Lanzanetti et al., 2004; Subramani and Alahari, 2010).

Growth factor stimulation of receptor tyrosine kinases promotes the activation of Rab5 through GEFS such as Rin1 or Rabex5 and promotes the formation of invasive cell membrane protrusions known lamellipodia as well as the formation of circular dorsal ruffles in a Rab5 dependent manner. Internalized early endosomes containing activated receptors and integrin complexes are associated with active Rab5 which subsequently recruits other Rab and adaptor/effector proteins to facilitate the trafficking of endosomal cargo. The small GTPase Rac is one such protein recruited to Rab5 positive early endosomes. Rab5 activates the Rac GEF Tiam1 which in turn activates Rac and promotes 
the remodeling of the actin cytoskeleton to form lamellipodia and circular dorsal ruffles (Zech and Machesky, 2008). Rabs 4 and 11 are also recruited to Rab5 positive early endosomes and facilitate the recycling of integrin complexes back to the plasma membrane as new focal adhesions are formed (Mendoza et al., 2014).

One of the early steps in the acquisition of a motile phenotype in epithelial cells is the loss of cell junctions that are responsible for maintaining epithelial cells in a polarized, growth arrested state. The cells of epithelial tissues are highly polarized meaning that they have distinct basal, lateral, and apical surfaces characterized by the specialized location of specific membrane proteins in these regions (Mosesson et al., 2008). The loss of junctional complexes such as tight junctions or adherens junctions along the basolateral surfaces of epithelial cells is often a precursor to cancer cell migration. Disruption of the endocytic machinery required to maintain tight junctions, for instance, typically involves mutations in the Par family of polarity genes as well as mutations in an atypical protein kinase $\mathrm{C}$ (aPKC) (Mosesson et al., 2008). As a consequence, tight junctional proteins are then abnormally targeted to lysosomes for degradation. The loss of E-cadherin from adherens junctions occurs through a similar mechanism involving mutation of the p120-Catenin protein that prevents E-cadherin endocytosis and degradation. Inactivation of p120-Catenin permits tyrosine phosphorylation of E-cadherin by Src which in turn promotes ubiquitination of E-cadherin by the Cbl-like ubiquitin ligase known as Hakai. The Ecadherin is subsequently targeted to lysosomes for degradation as a result of these modifications (Mellman and Yarden, 2013).

The connection of Rab5 with cancer cell invasion and migration has been documented for a variety of different cancers. Igarashi et al. (2017) report that Rab5 overexpression in 
pancreatic cancer samples correlated with increased lymphatic invasion and reduced expression of E-cadherin. A reversal of these oncogenic properties was observed with Rab5 inactivation. Similarly, Zhao et al. (2010) observed that Rab5A was significantly overexpressed in ovarian cancer samples when compared to benign cysts; and breast cancer samples that overexpress Rab5A also display a strong tendency to metastasize to axillary lymph nodes (Yang et al., 2011). Additionally, hypoxia has been shown to cause the activation and translocation of Rab5 to focal adhesions as well as significantly enhance the migratory behavior of B16-F0 murine melanoma cells (Silva et al., 2016).

Recent evidence also suggests that Rab5A in breast cancer promotes the invasive remodeling of the extracellular matrix by stimulating a Rab4 dependent fast recycling of membrane type 1 matrix metalloproteinase (MT1-MMP) and $\beta 3$ integrin (Frittoli et al., 2014). The MT1-MMP has been shown to coimmunoprecipitate and colocalize with $\beta 1$ integrins at regions of intercellular contact such as focal adhesions (Tang and $\mathrm{Ng}, 2009$ ). A similar study by Torres et al. (2009) reveals that caspase- 8 expression increases cell migration in A549 lung cancer cells through the Rab5 dependent internalization and recycling of $\beta 1$ integrins. Knockout of Rab5 inhibited the caspase- 8 induced activation of Rac and decreased cell migration. Interestingly, in a study on non-small cell lung cancer, Liu et al. (2015) report that knockdown of the CMTM7 tumor suppressor gene results in reduced Rab5 activation and prolonged EGFR signaling- presumably as a consequence of reduced EGFR internalization.

The tumorigenic properties of Rab5 are intrinsically linked to its ability to influence signaling pathways through its association with specific effector proteins. Principal among these effectors are the lipid modifying enzymes known as phosphatidylinositol-3 kinases 
(PI3Ks) (Chistoforidis et al., 1999). The PI3Ks are well known for their roles in signal transduction following receptor tyrosine kinase activation as well as membrane transport and cytoskeletal remodeling. Christoforidis et al. (1999) demonstrate that PI3Ks are recruited to Rab5 positive early endosomes and that their activity is required for Rab5 mediated docking and fusion of early endosomes. The PI3Ks are recruited to early endosomes by active Rab5 and then modify endosomal membrane lipids in a manner specified by Rab5 to permit the recruitment of other Rab5 effector proteins. One such Rab5 effector protein, EEA1, binds to the phosphatidylinositol lipids generated by PI3Ks and is necessary for endosome docking and fusion.

The signaling role of Rab5 in oncogenesis is further exemplified by the Rab5 mediated transport of APPL1 and APPL2 into the nucleus following growth factor stimulation. Both proteins are located on a specific population of Rab5 positive endosomes and APPL1 interacts with chromatin remodeling proteins in the nucleus to promote cell proliferation (Miaczynska et al., 2004). Similar Rab5 mediated endosomal trafficking of the MUC1 transmembrane glycoprotein has also been reported. MUC1 is frequently overexpressed in adenocarcinomas and its cytoplasmic tail has been implicated in tumor cell proliferation. Liu et al. (2008) report that overexpression of Rab5 in Chinese hamster ovary (CHO) cells results in the accumulation of MUC1 containing endosomes in the perinuclear region and that expression of the inactive Rab5 mutant (S34N) results in a more cytoplasmic distribution of MUC1.

Clearly, aberrant endocytosis has emerged as an important cause of tumorigenesis. Rab5 mediated cancer cell migration as a function of altered endocytosis has been identified as a major contributor to tumor progression. Defective growth factor receptor internalization 
and recycling, focal adhesion turnover, cell junction loss, and abnormal endosomal signaling have all been associated with the oncogenic capacity of Rab5. The significance of Rab5 mediated endocytosis to cancer cell development is clear, and future research into the key role that endocytosis plays in cancer biology will undoubtedly reveal novel therapeutic targets.

\subsection{The Mitogen Activated Protein Kinase Pathway and Cancer}

Many cancerous cells display abnormalities in the signal transduction pathways responsible for responding to extracellular growth factors, or mitogens. As reviewed above, mutations that alter proteins involved in these types of pathways can lead to inappropriate or unregulated cell growth and therefore predispose the cell to becoming cancerous. Hanahan and Weinberg (2000) in a comprehensive review of the molecular hallmarks of cancer cells list growth factor self-sufficiency as one of the six necessary physiological changes needed to convert a normal cell into a malignant cell. The authors also suggest that malignancy is acquired through a series of successive mutations which fall under two broad categories- dominant gain of function oncogenic mutations and recessive loss of function tumor suppressor mutations.

Unlike normal cells which will only grow and divide in response to external growth signals, many cancer cells have acquired the ability to proliferate in the absence of extracellular mitogenic signals. The apparent autonomy is caused by three key factors: selfproduction and stimulation by growth factors in an autocrine fashion, mutant growth factor cell surface receptors, and mutant intracellular signaling proteins (Hanahan and Weinberg, 2000). It is this third category of deregulation that appears to be found ubiquitously in most tumors and to which mutants of the Ras/MAPK pathway fall under. The critical role of the 
Ras/MAPK pathway in transducing growth signals to the interior of the cell and subsequently stimulating cell growth and proliferation is underscored by the fact that roughly one quarter of all human tumors contain mutant forms of Ras proteins (Hanahan and Weinberg, 2000). Therefore, more detailed discussion of the significance of the MAPK pathway to cancer development is warranted in an effort to convey a more comprehensive understanding of the tumorigenic process.

\subsection{A. Signal Transduction through the MAPK Pathway}

At this point it would be helpful to review the major steps of the RAS/MAPK pathway before proceeding to a more detailed consideration of the roles that downstream mediators of this widespread and critical cell signaling pathway play in cancer cell biology. The Ras/MAPK pathway is one of the principal means by which extracellular, mitogenic signals are transduced from the surface of the cell to the cell's interior (Fang and Richardson, 2005). The response often culminates in the nucleus with the transcription and expression of target genes which regulate cellular proliferation, differentiation, and development (Seger and Krebs, 1995; Wang et al., 2002). Growth factors, such as epidermal growth factor or insulin-like growth factor I, represent a major class of mitogenic ligands which can initiate the Ras/MAPK pathway.

The binding of a particular growth factor to its transmembrane receptor (tyrosine kinase receptor) sets into motion a series of events which results in the activation of several different cytoplasmic protein kinases. Receptor activation by binding of a growth factor ligand induces dimerization followed by auto-transphosphorylation of specific tyrosine residues within the receptor. These phospho-tyrosine residues are then recognized and bound by an adapter protein such as Grb2 through its $\mathrm{SH} 2$ domains (Seger and Krebs, 
1995). The small monomeric GTPase Ras is then activated through a guanine nucleotide exchange factor (GEF) protein known as Sos. Sos, in turn, is activated by the Grb2 adaptor protein. Activation of Ras through exchange of GDP for GTP then initiates the activation of a cascade of cytoplasmic serine-threonine protein kinases which include RAF1, MEK, and ultimately ERK (extracellular-signal regulated kinase). Each of these kinases is activated in sequential order by the protein kinase immediately preceding it in the pathway. Once phosphorylated and activated by MEK, ERK then proceeds to activate a number of cytoplasmic and nuclear proteins involved in regulating cell growth and proliferation. The regulation often occurs at the level of transcription.

One of the primary targets of activation by ERK are various types of transcription factors, including the E-twenty six (Ets) family of transcription factors as well as cMyc and cFos (Fang and Richardson, 2005). These transcription factors are potent stimulators of cellular proliferation (Seger and Krebs, 1995). Mut et al. (2011) provide evidence of the importance of the Ras/MAPK pathway in the activation of the E-twenty six like transcription factor 1 (Elk 1) in U138 glioblastoma multiforme cells. The researchers demonstrate that these cells have a high basal proliferative rate which can be reduced in the presence of specific MEK or ERK inhibitors. The authors hypothesize that inhibition of the Ras/MAPK pathway with these types of enzymatic inhibitors prevents the ultimate phosphorylation and activation of Elk 1, which in turn prevents the transcription of specific early cellular proliferation genes such as cFos. Activated Elk 1 exerts its effect in the nucleus by binding to the promoters of genes containing a serum response element (SRE) motif. The expression of a number of important cell cycle stimulatory genes, such as Egr1 and cFos, can be stimulated by Elk 1 . The results also indicate that stimulation with EGF 
results in a corresponding increase in the proliferative rate, which is most likely mediated by Elk 1 (Mut et al., 2011). For example, knockdown of Elk 1 through the use of siRNA does not result in an increase in proliferation even after EGF stimulation in U138 cells. The results also suggest that the PI3K/Akt pathway plays an important role in the regulation of Elk 1 activity. Inhibitors of this pathway do not prevent the phosphorylation of either ERK or Elk 1 in the cytoplasm following EGF stimulation, but rather prevent the movement of these phosphorylated proteins from the cytoplasm into the nucleus (Mut et al., 2011). The step is obviously necessary to produce the full mitogenic response to EGF stimulation.

In an intriguing study examining the role of protein kinase D1 (PKD1) in MCF-7 adenocarcinoma breast cancer cells, Karam et al. (2012) report that overexpression of PKD1 is associated with proliferation and various other aspects of tumorigenesis. When exposed to pharmacological inhibitors of PKD1, proliferation was reduced to levels measured in untreated control cells which do not overexpress PKD1. The result was further confirmed through the use of siRNA directed against PKD1, which produced a similar reduction in proliferation as measured by an MTT assay. A major finding of the study was that the level of phosphorylated ERK 1/2 was significantly higher in cells overexpressing PKD1 as compared to control cells not overexpressing the enzyme. The result was attributed to PKD1 overexpression since selective inhibition of the enzyme completely reversed the observed increase in ERK phosphorylation. Overexpression of PKD1, however, does not appear to affect levels of phosphorylated Akt. The amount of phosphorylated Akt was identical in both control cells and cells overexpressing PKD1, with or without exposure to PKD1 inhibitors (Karam et al., 2012). The investigators also report that inhibition of either MEK or ERK slowed the proliferation rate of MCF-7 cells 
overexpressing PKD1 as well as inhibited anchorage independent growth. These effects were observed to be dose dependent, with increasing amounts of MEK/ERK inhibitors slowing both the growth and proliferative capacity of the MCF-7 cells to the point where they began to resemble the non-overexpression control cells with respect to growth and proliferation. The authors also conclude that cells overexpressing PKD1 entered into S phase more rapidly and had a reduced dependence on serum for stimulation of proliferation when compared to control cells (Karam et al., 2012).

\subsection{B. Cancer and the MAPK Pathway}

Given the central role of the Ras/MAPK pathway in the proliferative response of cells to growth signals, it is logical that abnormalities in any one of its molecular components could lead to the uncontrolled cellular division characteristic of so many different cancers. In a review article detailing the molecular mechanisms underlying colorectal cancer, Fang and Richardson (2005) discuss the significance of the Ras/MAPK pathway in promoting growth, proliferation, and tumorigenesis in intestinal epithelial cells. Indeed, the importance of abnormalities in the MAPK pathway in promoting tumorigenesis is highlighted by the fact that this pathway is deregulated in about $30 \%$ of all cancers (Fang and Richardson, 2005). Abnormal MAPK signaling in colorectal cancer typically begins at the surface of the cell with overexpression and activation of EGF receptors. Additionally,

protein kinase $\mathrm{C}(\mathrm{PKC})$, when activated, can also facilitate the binding of GTP to Ras which ultimately leads to activation of the MAPK pathway.

Like many other types of cancers, the development of colorectal cancer is a multi-step process involving mutations in specific cell cycle regulatory or signaling genes. Mutations in Ras, specifically K-Ras, are known to be an early step in colorectal carcinogenesis along 
with mutations in certain protein kinases such as BRAF (Fang and Richardson, 2005). Hyperactive MAPK signaling through EGFR overexpression or mutant protein kinases in turn leads to the activation of various transcription factors, as mentioned earlier, which promote cell growth and proliferation. Additionally, unusual MPAK signaling can also induce the expression of VEGF which aids in tumor invasiveness and metastasis by promoting angiogenesis. Invasiveness of colorectal cancer might also be linked to increased synthesis of matrix metalloproteinases such as MMP7, which has been associated with abnormal MAPK signaling (Fang and Richardson, 2005). Given the key oncogenic role of the Ras/MAPK pathway in a variety of cancers, it is no surprise that a number of MEK and ERK inhibitors are currently being tested as possible chemotherapeutic agents.

\subsection{Rin1 as a MAPK Effector}

Besides anomalous cell signaling through the Ras/MAPK pathway, there is a growing body of evidence which suggests that specific Ras interactor proteins such as Rin1 (Ras and Rab Interactor protein 1) might be critical in modulating the cell's response to mitogenic signals (Wang et al., 2002). The ability of Rin1 to have a moderating effect on signal transduction through the Ras/MAPK pathway lies in its ability to be activated by Ras. In fact, Rin1 has been shown to compete directly with RAF1 for activation by Ras (Wang et al., 2002) and this competition has important implications for cancer biology research. For instance, could Rin1 be a useful target to help dampen or modify excessive signaling through the Ras/MAPK pathway in certain cancer cell lines? If so, could one then reduce the downstream proliferative response induced by the Ras/MAPK pathway as a result of growth factor stimulation? Before addressing these possibilities, it is necessary to first review the biochemical role of Rin1 in cells. 
Han et al. (1997) and Wang et al. (2002) studied the biochemical properties of Rin1 and report that Rin1 is an important downstream effector of activated Ras. Rin1 is able to bind to Ras through its Ras binding domain (RBD) which associates with an effector domain within Ras. Furthermore, biochemical analysis reveals that Rin1 has a high binding affinity for activated Ras and that it competes strongly with RAF1 for access to Ras. Molecular studies also indicate that Rin1 binds to $14-3-3$ proteins in the cytoplasm and is able to interact with and be phosphorylated by the tyrosine kinase c-ABL.

The ability of Rin1 to bind to Ras lies in its carboxyl-terminal domain which contains a 433 amino acid sequence (between residues 294 to 727) necessary for Ras binding (Han et al., 1997). Different Ras effector proteins, such as Rin1 and RAF1, can vary significantly in terms of their overall primary structure but often display a high degree of similarity in the regions required for Ras binding (Ras binding domains). The Ras binding domains of effector proteins typically interact with a short amino acid effector sequence within Ras itself, and this interaction is heavily dependent on Ras being in its activated GTP-bound state. For Rin1, the carboxyl-terminal domain (Rin1C) mediates binding to both GTP-Ras and to 14-3-3 proteins (Wang et al., 2002).

The affinity of Rin1 for Ras can be seen in experiments utilizing antibodies against Ras. For example, Rin1 is co-immunoprecipitated with an overly active mutant allele of H-Ras in NIH 3 T3 cells when treated with a particular Ras antibody. When the same antibody is pre-attached to Ras, Rin1 is not co-immunoprecipitated. The use of antibodies which bind to the switch II region of Ras also prevent the binding of Rin1. Additionally, the effector binding domain within Ras itself is equally important in mediating the proper attachment of certain effector proteins to Ras (Han et al., 1997). The point is illustrated by the fact that 
mutations in the Ras effector binding domain can selectively inhibit the binding of certain Ras effector proteins without affecting the binding of others. Rin1, for instance, can interact with a constitutively active mutant form of $\mathrm{H}-\mathrm{Ras}\left(\mathrm{H}-\mathrm{Ras}^{\mathrm{V} 12}\right)$. However, when the mutant form of H-Ras acquires additional mutations at amino acid positions 35 or 40, Rin 1 binding is completely inhibited. The position 35 mutation on the other hand does not interfere with RAF1 binding, while a mutation at position 37 permits Rin 1 association but prevents RAF1 binding (Han et al., 1997). The ability of full length Rin1 to interact with Ras is further highlighted by a variant form of Rin1 in which there is a 62 amino acid deletion. The naturally occurring truncated form of Rin1, known as Rin1 delta, is the result of alternative splicing of the Rin1 mRNA. Rin1 delta exhibits a much weaker binding affinity for Ras when compared to full length Rin1 (Han et al., 1997).

Besides interaction with Ras, the carboxyl-terminal domain of Rin1 also mediates binding to 14-3-3 proteins (Han et al., 1997; Wang et al., 2002). These small acidic proteins exist as multiple isoforms in the cytoplasm (epsilon, beta, and zeta) and have been shown to be involved in mitogenesis and malignant transformation of cells through their interactions with signaling proteins. Both RAF1 and Rin1 share the ability to bind to 14-33 proteins via their Ras binding domains, and this binding typically results in the activation of RAF1 through a Ras-dependent mechanism that seems to enhance signal transduction functions (Wang et al., 2002). Since Rin1 competes directly with RAF1 for access to Ras, the binding of Rin1 to 14-3-3 proteins would reduce its potential to bind to Ras and thus allow for heightened signaling through the Ras/MPAK pathway. The binding of Rin1 to cytoplasmic 14-3-3 proteins would promote the oncogenic properties of cell growth and proliferation as a result of the increased access of RAF1 over Rin1 for activation by Ras. 
Interestingly, deletion of the Ras binding domain within the carboxyl-terminal of Rin1 prevents Rin1 from binding to all isoforms of 14-3-3 proteins, as does the 62 amino acid deletion in the naturally occurring Rin1delta (Wang et al., 2002).

The interaction of Rin1 with 14-3-3 proteins appears to be largely controlled by a serine residue at position 351 within the Ras binding domain. The serine residue is phosphorylated predominantly by protein kinase $\mathrm{D}$ and its phosphorylation is required for proper binding to 14-3-3 proteins (Wang et al., 2002). A mutation which substitutes alanine at this position blocks interaction with $14-3-3$ proteins and results in an increased ability to suppress signaling through the Ras/MAPK pathway, presumably caused by an increased capacity to compete with RAF1 for access to Ras. The phosphorylation of serine 351 of Rin1 and subsequent attachment to 14-3-3 proteins may act as a suppression control mechanism in cells to unlink Rin 1 from activated Ras by sequestering it in the cytoplasm (Wang et al., 2002).

An important point is raised here: in order for Rin1 to effectively compete with RAF1 for access to Ras it must be in the proper subcellular location. Wild type Rin1 in its nonphosphorylated state is weakly associated with the cell membrane and is in close proximity to interact with Ras, which is tightly associated with the plasma membrane. Also, the alanine substitution at position 351 in the mutant form of Rin1 allows for a significant shift to the plasma membrane which may help to explain its suppressive effect on Ras signaling (Wang et al., 2002). However, when phosphorylated by PKD, wild type Rin1 is confined to the cytoplasm bound to 14-3-3 proteins. The phosphorylation of Rin1 by PKD therefore reduces its capacity to compete with RAF1 for binding to Ras. 
The amino-terminal of Rin1, like the carboxyl-terminal, plays an important role in mediating cell signaling through its ability to bind to the tyrosine kinase c-ABL (ABL1). ABL tyrosine kinases are known to be involved in various cellular functions including differentiation, division, migration, and adhesion ( $\mathrm{Hu}$ et al., 2005). Additionally, the amino-terminal of Rin1 contains an SH2 domain capable of interacting with phosphotyrosine residues on an activated receptor tyrosine kinase such as the EGFR (Barbieri et al., 2004). Rin1 interacts with c-ABL most likely through a proline rich sequence in its amino terminal and an SH3 domain in c-ABL (Han et al., 1997). Upon binding to c-ABL in vitro, Rin1 becomes tyrosine phosphorylated and can then subsequently bind to an $\mathrm{SH} 2$ domain within c-ABL. The interaction does not seem to affect the catalytic activity of the enzyme however, and studies have shown that Rin1 has very little interaction with c-ABL in vivo. This reduced interaction is caused in part by the different cellular locations of the two proteins; with c-ABL being confined mostly to the nucleus and Rin1 to the cytoplasm (Han et al., 1997).

One interesting exception to this is the oncogenic BCR/ABL fusion protein produced as a result of a translocation between chromosomes 9 and 22. The BCR/ABL is an unregulated tyrosine kinase which is localized primarily to the cytoplasm where it stimulates cellular proliferation. The BCR/ABL is therefore in the correct location to interact with Rin1 and, indeed, Rin1 is able to bind to BCR/ABL. Rin1 appears to accentuate the tumorigenic, transforming properties of BCR/ABL (Wang et al., 2002; $\mathrm{Hu}$ et al., 2005; Afar et al., 1997). Additionally, Hu et al. (2005) report that Rin 1 is an activator of the ABL2 tyrosine kinase which is involved in the regulation of epithelial cell adhesion and migration. Specifically, Rin1 activation of ABL2 promotes phosphorylation of the 
adaptor proteins CRK and CRKL. This phosphorylation in turn produces conformational changes in CRK and CRKL which influence cytoskeletal elements to inhibit cell motility. Cells deficient in Rin1 display reduced levels of phosphorylated CRKL and increased cell motility (Hu et al., 2005).

Clearly, the biochemical profile of Rin 1 suggests that it potentially plays a key role in modulating signaling through the Ras/MAPK pathway given its ability to interact with multiple different effector proteins. The greatest capacity for Rin1 to moderate abnormal signaling, as detailed above, appears to lie in its ability to directly compete with RAF1 for access to Ras. The competition, in turn, could be a useful mechanism for dampening or attenuating signaling through the Ras/MAPK pathway by diverting the signal away from effector proteins downstream of Ras. On the contrary, elevated levels of Rin1 may be associated with increased tumorigenesis and lower survival as is reported by Wang et al. (2012) for non-small cell lung cancer. It should be noted that although Rin1 is thought to be expressed in most tissues, its expression is highest in brain tissues (Han et al., 1997). The differential level of Rin1 expression could have important implications for research when investigating Rin1 function in various tissues and/or cell lines.

\subsection{Rin1 and Rab5 in the Context of the MAPK Pathway}

Before delving any further into the important role that Rin1 plays in specific cancers, it will be useful to briefly review another significant function of Rin1 in most cells. Rin1, in addition to its ability to moderate mitogenic signaling through the Ras/MAPK pathway, also acts as a guanine nucleotide exchange factor (GEF) for the small monomeric GTPase known as Rab5 (Tall et al., 2001). The Rab proteins are a diverse group of proteins which belong to the Ras superfamily of small GTPases and play critical roles in regulating the 
steps of endocytic vesicular transport. Specifically, Rab proteins regulate vesicular traffic from the plasma membrane by controlling cargo selection, vesicle formation, transport along the cytoskeleton, and fusion with intracellular target membranes (Stein et al., 2003; Hutagalung and Novick, 2011; Stenmark and Olkonnen, 2001). Certain Rab proteins also control the sorting of molecules for return to the plasma membrane or for degradation in lysosomes. The ability of Rab proteins to regulate the many complex steps of vesicular transport lies in their selective activation. There are approximately 60 different Rab proteins encoded in the human genome, each of which is selectively activated by binding GTP. Once activated, a particular Rab protein serves as a scaffold for the attachment of various effector proteins which then subsequently direct the completion of a specific step in the endocytic pathway.

Rab5, which is activated by Rin1, has been shown to regulate vesicle budding and cargo selection from clathrin coated pits as well as early endosome fusion (Stein et al., 2003; Hutagalung and Novick, 2011; Stenmark and Olkonnen, 2001). Tall et al. (2001) report that Rin1 and Rab5 also play a crucial role in the receptor mediated endocytosis of epidermal growth factor receptor (EGFR) following stimulation by EGF. The receptor mediated endocytosis of EGFR occurs through a Ras mediated mechanism involving upstream activation of Rin1 by GTP-Ras and then subsequent activation of Rab5 by activated Rin1.

The pivotal integrative role of Rab proteins in signal transduction is further illustrated in a study by Barbieri et al. (2004) examining the role of Rab5 in EGFR mediated MAPK signal transduction. Rin1 interacts with Rab5 via its Vps9 domain which also contains the GEF activation function for Rab5. Galvis et al. (2009) have previously reported the key 
function of the Vps9 domain of Rin1 in activating Rab5 through a series of mutational studies. Barbieri et al. (2004) demonstrate that a dominant negative mutant form of Rab5 (Rab5:S34N) is capable of inhibiting activation of the MAPK pathway in mouse NR6 cells by preventing both the endocytosis and internalization of the EGFR. The inhibition is particular to the MAPK pathway and does not interfere with other EGF induced kinase pathways.

A similar inhibitory effect is observed with the overexpression of wild type full-length Rin1. On the other hand, however, expression of wild type Rab5 or the Rin1 delta splice variant leads to increased MAPK activity and increased cyclin D1 expression after EGF stimulation- resulting in heightened cellular proliferation. The authors suggest that Rab5 activation is a key step in linking EGF stimulated endocytosis to signal transduction through the MAPK pathway (Barbieri et al., 2004). Although the exact biochemical link between Ras association and the Rab5 GEF activity of Rin1 is not entirely clear, the binding of Ras by Rin1 appears to strongly influence EGFR endocytosis.

In a manner similar to EGFR endocytosis, the internalization of insulin receptor following the binding of insulin may involve other steps including Rab5 activation by Rin1 (Hunker et al., 2005). It is interesting to note that Rin1 sits at the intersection between cell signaling and receptor mediated endocytosis for mitogens such as EGF and insulin. The increased rate of receptor mediated endocytosis potentiated by Ras stimulation of the Rab5 GEF activity of Rin1 may be an important negative feedback mechanism by which Ras can divert signaling away from downstream effectors through Rin1. In this model, an increased rate of receptor mediated endocytosis would favor quick removal of receptors from the plasma membrane followed by internalization and degradation (Hunker et al., 2005). The 
end result would be an attenuation of mitogenic signal transduction much in the same way as can be achieved by the direct competition of Rin1 with RAF1 for access to Ras.

Having examined the biochemical properties of Rin1 and how it fits into the larger signal transduction machinery of the Ras/MAPK pathway, it would be useful to elaborate on the possible connection between Rin1 and specific downstream effectors of the Ras/MAPK pathway known to induce cellular proliferation. These downstream targets, while varied, include the Ets family of transcription factors mentioned earlier as well as the telomerase reverse transcriptase enzyme. It is intriguing to wonder if Rin1, given its ability to compete directly with RAF1 for access to Ras, could have any influence on the cellular proliferation associated with classical MAPK targets such as the Ets transcription factors and/or telomerase.

The signaling pathways that have been implicated in the stimulation of telomerase expression and activity usually involve the response to a mitogen such as EGF or IGF-1, and subsequent activation of a number of protein kinases belonging to the Ras/MAPK and PI3K/Akt pathways (Inui et al., 2002; Seimiya et al., 1999; Zhou et al., 2013). It is feasible then that Rin 1 could potentially have an effect on telomerase expression given its role as a Ras effector molecule. If so, this raises the exciting idea of Rin1 as a potential therapeutic target in specific cancers. To appreciate the role of Rin1 in a therapeutic context, it is necessary to review what effect the regulation of the telomerase reverse transcriptase enzyme and the Ets family of transcription factors have on cellular proliferation.

In both normal and cancerous cells, Ras effector proteins such as Rin1 may be useful targets for modulation of signaling through the MAPK pathway. The detrimental effects of abnormalities in the Ras/MAPK pathway are well documented for a variety of different 
cancers. Cancer, in the simplest sense, is a disease of the cell cycle and dissection of the complex molecular interactions which govern cancer cell growth and reproduction will ultimately shed light on instances where moderation of aberrant cell signaling may be possible. Rin1, a known Ras effector molecule, may provide an avenue for attenuation of signaling through the Ras/MAPK pathway. If so, it is intriguing to contemplate what effect molecules such as Rin1 could have on downstream targets of the MAPK pathway known to influence cellular proliferation. Telomerase, for example, is widely expressed in many cancers and has been heavily implicated in tumorigenesis. As a target of the Ras/MAPK pathway, it is interesting to speculate on a possible connection between telomerase expression and the modifying ability of a Ras effector such as Rin1.

\subsection{Specific Aims}

Aim I

Does the expression of Rin1 affect telomerase gene expression and enzymatic activity in breast cancer cells in response to growth factor stimulation, and is there a correlation with cellular proliferation? The first specific aim of the research focuses on examining the effect of Rin1 expression on telomerase gene expression and activity in normal breast epithelial cells as well as in tumorigenic, non-metastatic and metastatic breast cancer cells. The effect of Rin1 expression on cellular proliferation will also be examined.

Aim II

Does the expression of different Rin1 constructs affect telomerase protein expression and enzymatic activity in breast cancer cells? The second specific aim of the research seeks to determine the effect of various Rin 1 constructs on telomerase protein 
expression and activity in a tumorigenic, metastatic breast cancer cell line. The cellular mechanism underlying the influence of Rin1 on telomerase protein expression and activity will also be examined.

Aim III

Does the expression of Rin1 affect telomerase activity in other cancer cell lines?

The third specific aim of the research evaluates the role of Rin1 on telomerase activity in different cancer cell lines, including human glioblastoma and melanoma cell lines upon growth factor stimulation. 


\subsection{References}

Adotevi, O., Mollier, K., Neuveut, C., Dosset, M., Ravel, P., Tartour, E., \&LangladeDemoyen, P. (2010). Targeting human telomerase reverse transcriptase with recombinant lentivector is highly effective to stimulate antitumor CD8 T-cell immunity in vivo. Blood, 115(15), 3025-3032.

Afar, D. E., Han, L., McLaughlin, J., Wong, S., Dhaka, a, Parmar, K., \& Colicelli, J. (1997). Regulation of the oncogenic activity of BCR-ABL by a tightly bound substrate protein RIN1. Immunity, 6(6), 773-82.

Barbieri, M. A., Fernandez-Pol, S., Hunker, C., Horazdovsky, B. H., \& Stahl, P. D. (2004). Role of rab5 in EGF receptor-mediated signal transduction. European Journal of Cell Biology, 83(6), 305-14.

Beisner, J., Dong, M., Taetz, S., Nafee, N., Griese, E.-U., Schaefer, U., \& Mürdter, T. E. (2009). Nanoparticle mediated delivery of 2'-O-methyl-RNA leads to efficient telomerase inhibition and telomere shortening in human lung cancer cells. Lung Cancer, 68, 346-54.

Bernardes de Jesus, B., \& Blasco, M. A. (2013). Telomerase at the intersection of cancer and aging. Trends in Genetics, 29(9), 513-520.

Buseman, C. M., Wright, W. E., \& Shay, J. W. (2012). Is telomerase a viable target in cancer. Mutation Research, 730(1-2), 90-97.

Cerone, M. A., Burgess, D. J., Naceur-Lombardelli, C., Lord, C. J., \& Ashworth, A. (2011). High-throughput RNAi screening reveals novel regulators of telomerase. Cancer Research, 71(9), 3328-3340.

Chen, C.H., \& Chen, R.J. (2011). Prevalence of telomerase activity in human cancer. Journal of the Formosan Medical Association, 110(5), 275-289.

Chen, H., Landen, C., Li, Y., Alvarez, R., \& Tollefsbol, T. O. (2013). Epigallocatechin gallate and sulforaphane combination treatment induce apoptosis in paclitaxelresistant ovarian cancer cells through hTERT and Bcl-2 down-regulation. Exp Cell Res., 319(5), 697-706.

Christoforidis, S., Wilm, M., Yip, S., Zerial, M., Zhao, L., Miaczynska, M., \& Waterfield, M. D. (1999). Phosphatidylinositol-3-OH kinases are Rab5 effectors. Nature Cell Biology, 1(4), 249-252. 
de Launoit, Y., Baert, J. L., Chotteau-Lelievre, A., Monte, D., Coutte, L., Mauen, S., \& Verreman, K. (2006). The Ets transcription factors of the PEA3 group: transcriptional regulators in metastasis. Biochimica et Biophysica Acta, 1766(1), 7987.

Deeb, D., Gao, X., Liu, Y., Pindolia, K. R., Arbab, A. S., \& Gautam, S. C. (2012). Inhibition of cell proliferation and induction of apoptosis by oleanane triterpenoid (CDDO-Me) in pancreatic cancer cells is associated with the suppression of hTERT gene expression and its telomerase activity. Biochem Biophys Res Commun., 422(4), $561-567$.

Dwyer, J., Li, H., Xu, D., \& Liu, J.-P. (2007). Transcriptional regulation of telomerase activity: roles of the the Ets transcription factor family. Annals of the New York Academy of Sciences, 1114, 36-47.

Fang, J. Y., \& Richardson, B. C. (2005). The MAPK signaling pathways and colorectal cancer. Lancet Oncology, 6(5), 322-327.

Flavin, P., Redmond, A., McBryan, J., Cocchiglia, S., Tibbitts, P., Fahy-Browne, P., \& Young, L. S. (2011). RuvB12 cooperates with Ets2 to transcriptionally regulate hTERT in colon cancer. FEBS Letters, 585(15), 2537-2544.

Frittoli, E., Palamidessi, A., Marighetti, P., Confalonieri, S., Bianchi, F., Malinverno, C., \& Scita, G. (2014). A RAB5/RAB4 recycling circuitry induces a proteolytic invasive program and promotes tumor dissemination. The Journal of Cell Biology, 206(2), 307-328.

Gai, X., et al. (2013). Hepatitis B virus core protein enhances human telomerase reverse transcriptase expression and hepatocellular carcinoma cell proliferation in a c-Ets2dependent manner. International Journal of Biochemistry and Cell Biology, 45(7), 1174-1185.

Galvis, A., Balmaceda, V., Giambini, H., Conde, A., Villasana, Z., Fornes, M. W., \& Barbieri, M. A. (2009). Inhibition of early endosome fusion by Rab5-binding defective Ras interference 1 mutants. Archives of Biochemistry and Biophysics, 482(1-2), 83-95.

Galvis, A., Giambini, H., Villasana, Z., \& Barbieri, M. A. (2009). Functional determinants of ras interference 1 mutants required for their inhbitory activity on endocytosis. Experimental Cell Research, 315(5), 820-35.

Gandellini, P., Folini, M., Bandiera, R., De Cesare, M., Binda, M., Veronese, S., \& Zaffaroni, N. (2007). Down-regulation of human telomerase reverse transcriptase through specific activation of RNAi pathway quickly results in cancer cell growth impairment. Biochemical Pharmacology, 73, 1703-1714. 
Gomez, D. E., Armando, R. G., Farina, H. G., Menna, P. L., Cerrudo, C. S., Ghiringhelli, P. D., \& Alonso, D. F. (2012). Telomere structure and telomerase in health and disease. International Journal of Oncology, 41, 1561-1569.

Goueli, B. S., \& Janknecht, R. (2004). Upregulation of the Catalytic Telomerase Subunit by the Transcription Factor ER81 and Oncogenic HER2/Neu, Ras, or Raf. Molecular and Cellular Biology, 24(1), 25-35.

Han, L., Wong, D., Dhaka, A., Afar, D., White, M., Xie, W., \& Colicelli, J. (1997). Protein binding and signaling properties of RIN1 suggest a unique effector function. Proceedings of the National Academy of Sciences of the United States of America, 94(10), 4954-9.

Hanahan, D., \& Weinberg, R. (2000). The Hallmarks of Cancer. Cell, 100, 57-70.

Holysz, H., Lipinska, N., Paszel-Jaworska, A., \& Rubis, B. (2013). Telomerase as a useful target in cancer fighting-the breast cancer case. Tumor Biology, 34, 13711380.

Hsu, C. P., Hsu, N. Y., Lee, L. W., \& Ko, J. L. (2006). Ets2 binding site single nucleotide polymorphism at the hTERT gene promoter - effect on telomerase expression and telomere length maintenance in non-small cell lung cancer. European Journal of Cancer, 42, 1466-1474.

Hu, H., Bliss, J. M., Wang, Y., \& Colicelli, J. (2005). RIN1 is an ABL tyrosine kinase activator and a regulator of epithelial-cell adhesion and migration. Current Biology, 15(9), 815-823.

Hu, J., Hwang, S. S., Liesa, M., Gan, B., Sahin, E., Jaskelioff, M., \& DePinho, R. A. (2012). Anti-telomerase therapy provokes ALT and mitochondrial adaptive mechanisms in cancer. Cell, 148(4), 651-663.

Huang, D.-S., Wang, Z., He, X.-J., Diplas, B. H., Yang, R., Killela, P. J., \& Tao, H.Q. (2015). Recurrent TERT promoter mutations identified in a large- scale study of multiple tumour types are associated with increased TERT expression and telomerase activation. European Journal of Cancer, 51, 969-976.

Huang, F.C., Chang, C.C., Wang, J.M., Chang, T.C., \& Lin, J.J. (2012). Induction of senescence in cancer cells by the G-quadruplex stabilizer, BMVC4, is independent of its telomerase inhibitory activity. British Journal of Pharmacology, 167, 393406.

Hunker, C. M., Giambini, H., Galvis, A., Hall, J., Kruk, I., Veisaga, M. L., \& Barbieri, M. A. (2006). Rin1 regulates insulin receptor signal transduction pathways. Experimental Cell Research, 312(7), 1106-18. 
Hutagalung, A., \& Novick, P. (2011). Role of Rab GTPases in Membrane Traffic and Cell Physiology. Physiology Review, 91(1), 119-149.

Igarashi, T., Araki, K., Yokobori, T., Altan, B., Yamanaka, T., Ishii, N., \& Kuwano, H. (2017). Association of RAB5 overexpression in pancreatic cancer with cancer progression and poor prognosis via E-cadherin suppression. Oncotarget, 8(7), 12290-12300.

Inui, T., Shinomiya, N., Fukasawa, M., Kobayashi, M., Kuranaga, N., Ohkura, S., \& Seki, S. (2002). Growth-related signaling regulates activation of telomerase in regenerating hepatocytes. Experimental Cell Research, 273(2), 147-56.

Jaitner, S., Reiche, J. A., Schäffauer, A. J., Hiendlmeyer, E., Herbst, H., Brabletz, T., \& Jung, A. (2012). Human telomerase reverse transcriptase (hTERT) is a target gene of $\beta$-catenin in human colorectal tumors. Cell Cycle, 11(17), 3331-3338.

Karam, M., Legay, C., Auclair, C., \& Ricort, J. M. (2012). Protein kinase D1 stimulates proliferation and enhances tumorigenesis of MCF-7 human breast cancer cells through a MEK/ERK-dependent signaling pathway. Experimental Cell Research, $318(5), 558-569$.

Lanzetti, L., Di Fiore, P. P., Palamidessi, A., Scita, G., \& Areces, L. (2004). Rab5 is a signalling GTPase involved in actin remodelling by receptor tyrosine kinases. Nature, 429(6989), 309-314.

Liao, Z.L., Luo, G., Xie, X., Tang, X.D., Bai, J. Y., Guo, H., \& Yang, S.M. (2013). Diepitope multiple antigen peptide of hTERT trigger stronger anti-tumor immune responses in vitro. International Immunopharmacology, 16, 444-450.

Listerman, I., Gazzaniga, F. S., \& Blackburn, E. H. (2014). An investigation of the effects of the core protein telomerase reverse transcriptase on Wnt signaling in breast cancer cells. Molecular and Cellular Biology, 34(2), 280-289.

Liu, B., Su, Y., Li, T., Yuan, W., Mo, X., Li, H., \& Han, W. (2015). CMTM7 knockdown increases tumorigenicity of human non-small cell lung cancer cells and EGFR-AKT signaling by reducing Rab5 activation. Oncotarget, 6(38), 41092-41107.

Liu, J. P. (1999). Studies of the molecular mechanisms in the regulation of telomerase activity. FASEB Journal : Official Publication of the Federation of American Societies for Experimental Biology, 13(15), 2091-104.

Liu, S., Qi, Y., Ge, Y., Duplessis, T., Rowan, B. G., Ip, C., \& Dong, Y. (2010). Telomerase as an important target of androgen-signaling blockade for prostate cancer treatment. Mol Cancer Ther., 9(7), 2016-2025. 
Liu, X., Yuan, Z., \& Chung, M. (2008). MUC1 intra-cellular trafficking is clathrin, dynamin, and rab5 dependent. Biochemical and Biophysical Research Communications, 376(4), 688-693.

Liu, Y., Gao, X., Deeb, D., Arbab, A. S., \& Gautam, S. C. (2012). Telomerase reverse transcriptase (TERT) is a therapeutic target of oleanane triterpenoid CDDO-Me in prostate cancer. Molecules, 17, 14795-14809.

Lund, J. R., Paoloni, M., Kurzman, I., Padilla, M., \& Argyle, D. J. (2008). Inhibition of canine telomerase in vitro and in vivo using RNAi: further development of a natural canine model for telomerase-based cancer therapies. The Veterinary Journal, 177, $192-7$.

Maida, Y., Kyo, S., Kanaya, T., Wang, Z., Yatabe, N., Tanaka, M., \& Inoue, M. (2002). Direct activation of telomerase by EGF through Ets-mediated transactivation of TERT via MAP kinase signaling pathway. Oncogene, 21(26), 4071-4079.

Marian, C. O., Cho, S. K., Mcellin, B. M., Maher, E. A., Hatanpaa, K. J., Madden, C. J., \& Bachoo, R. M. (2010). The telomerase antagonist Imetelstat efficiently targets glioblastoma tumor-initiating cells leading to decreased proliferation and tumor growth. Clinical Cancer Research, 16(1), 154-163.

Mazumdar, T., Sandhu, R., Qadan, M., DeVecchio, J., Magloire, V., Agyeman, A., \& Houghton, J. A. (2013). Hedgehog signaling regulates telomerase reverse transcriptase in human cancer cells. Plos One, 8(9), e75253.

Mellman, I., \& Yarden, Y. (2013). Endocytosis and cancer. Cold Spring Harbor Perspectives in Biology, 5(12), a016949.

Mendoza, P., Diaz, J., Ortiz, R., Diaz, N., Leyton, L., Stupak, D., \& Torres, V. A. (2014). Rab5 is required in metastatic cancer cells for caveolin-1-enhanced Rac1 activation, migration and invasion. Journal of Cell Science, 127(11), 2401-2406.

Mendoza, P., Díaz, J., Silva, P., \& Torres, V. A. (2014). Rab5 activation as a tumor cell migration switch. Small GTPases, 5(1), e28195.

Mendoza, P., Díaz, J., \& Torres, V. A. (2014). On the role of Rab5 in cell migration. Current Molecular Medicine, 14(2), 235.

Miaczynska, M., Christoforidis, S., Giner, A., Shevchenko, A., Uttenweiler-Joseph, S., Habermann, B., \& Zerial, M. (2004). APPL proteins link Rab5 to nuclear signal transduction via an endosomal compartment. Cell, 116(3), 445-456.

Miyazaki, Y., Fujiwara, H., Asai, H., Ochi, F., Ochi, T., Azuma, T., \& Yasukawa, M. (2013). Development of a novel redirected T-cell-based adoptive immunotherapy targeting human telomerase reverse transcriptase for adult T-cell leukemia. Blood, 121(24), 4894-4901. 
Mohammad, R. Y., Somayyeh, G., Gholamreza, H., Majid, M., \& Yousef, R. (2013). Diosgenin inhibits hTERT gene expression in the A549 lung cancer Cell line. Asian Pacific Journal of Cancer Prevention, 14(11), 6945-6948.

Mosesson, Y., Mills, G. B., \& Yarden, Y. (2008). Derailed endocytosis: An emerging feature of cancer. Nature Reviews Cancer, 8(11), 835-850.

Mut, M., Lule, S., Demir, O., Kurnaz, I. A., \& Vural, I. (2011). Both mitogen-activated protein kinase (MAPK)/extracellular-signal-regulated kinases (ERK) 1/2 and phosphatidylinositide-3-OH kinase (PI3K)/Akt pathways regulate activation of Etwenty-six (Ets)-like transcription factor 1 (Elk-1) in U138 glioblastoma cell. The International Journal of Biochemistry \& Cell Biology, 44(2), 302-310.

Nasiri, M., Zarghami, N., Koshki, K. N., Mollazadeh, M., Moghaddam, M. P., Yamchi, M. R., \& Alibakhshi, A. (2013). Curcumin and silibinin inhibit telomerase expression in T47D human breast cancer cells. Asian Pacific Journal of Cancer Prevention, 14(6), 3449-3453.

Nicholls, C., Ruvantha Pinto, A., Li, H., Li, L., Wang, L., \& Simpson, R. (2012). Glycceraldehyde-3-phosphate dehydrogenase (GAPDH) induces cancer cell senescence by interacting with telomerase RNA component. Proceedings of the National Academy of Sciences of the United States of America, 109(33), 1330813313.

Rachakonda, P. S., Hosen, I., de Verdier, P. J., Fallah, M., Heidenreich, B., Ryk, C., \& Kumar, R. (2013). TERT promoter mutations in bladder cancer affect patient survival and disease recurrence through modification by a common polymorphism. Proceedings of the National Academy of Sciences of the United States of America, 110(43), 17426-17431.

Seger, R., \& Krebs, E. G. (1995). The MAPK signaling cascade. FASEB Journal : Official Publication of the Federation of American Societies for Experimental Biology, 9(9), 726-35.

Seimiya, H., Tanji, M., Oh-hara, T., Tomida, A., Naasani, I., \& Tsuruo, T. (1999). Hypoxia up-regulates telomerase activity via mitogen-activated protein kinase signaling in human solid tumor cells. Biochemical and Biophysical Research Communications, 260(2), 365-70.

Silva, P., Mendoza, P., Rivas, S., Díaz, J., Moraga, C., Quest, A. F. G., \& Torres, V. A. (2016). Hypoxia promotes Rab5 activation, leading to tumor cell migration, invasion and metastasis. Oncotarget, 7(20), 29548-29562. 
Stein, M. P., Dong, J., \& Wandinger-Ness, A. (2003). Rab proteins and endocytic trafficking: Potential targets for therapeutic intervention. Advanced Drug Delivery Reviews, 55(11), 1421-1437.

Stenmark, H., \& Olkkonen, V. M. (2001). The Rab GTPase family. Genome Biology, 2(5), 3007.1-3007.7.

Subramani, D. \& Alahari, S. (2010). Integrin-mediated function of rab GTPases in cancer progression. Molecular Cancer, 9(1), 312.

Tall, G. G., Barbieri, M. A., Stahl, P. D., \& Horazdovsky, B. F. (2001). Ras-Activated Endocytosis Is Mediated by the Rab5 Guanine Nucleotide Exchange Activity of RIN1. Developmental Cell, 1(1), 73-82.

Tang, B. L., \& Ng, E. L. (2009). Rabs and cancer cell motility. Cytoskeleton, 66(7), 365370.

Torres, V. A., Mielgo, A., Barbero, S., Hsiao, R., Wilkins, J. A., \& Stupack, D. G. (2010). Rab5 mediates caspase-8-promoted cell motility and metastasis. Molecular Biology of the Cell, 21(2), 369-376.

Uziel, O., Beery, E., Dronichev, V., Samocha, K., Gryaznov, S., Weiss, L., \& Lahav, M. (2010). Telomere shortening sensitizes cancer cells to selected cytotoxic agents: in vitro and in vivo studies and putative mechanisms. Plos One, 5(2), e9132.

Wang, Q., Gao, Y., Tang, Y., Ma, L., Zhao, M., \& Wang, X. (2012). Prognostic significance of RIN1 gene expression in human non-small cell lung cancer. Acta Histochemica, 114(5), 463-468.

Wang, Y., Waldron, R. T., Dhaka, A., Patel, A., Riley, M. M., Rozengurt, E., \& Colicelli, J. (2002). The RAS effector RIN1 directly competes with RAF and is regulated by 14-3-3 proteins. Molecular and Cellular Biology, 22(3), 916-926.

Wong, M. S., Chen, L., Foster, C., Kainthla, R., Shay, J. W., \& Wright, W. E. (2013). Regulation of telomerase alternative splicing: a new target for chemotherapy. Cell Rep., 3(4), 1028-1035.

Xia, W., Wang, P., Lin, C., Li, Z., Gao, X., Wang, G., \& Zhao, X. (2012). Bioreducible polyethylenimine-delivered siRNA targeting human telomerase reverse transcriptase inhibits HepG2 cell growth in vitro and in vivo. Journal of Controlled Release, 157, 427-436.

Xiao, X., Athanasiou, M., Sidorov, I. a., Horikawa, I., Cremona, G., Blair, D., \& Dimitrov, D. S. (2003). Role of Ets/Id proteins for telomerase regulation in human cancer cells. Experimental and Molecular Pathology, 75(3), 238-247. 
Xu, D., Dwyer, J., Li, H., Duan, W., \& Liu, J.P. (2008). Ets2 Maintains hTERT Gene Expression and Breast Cancer Cell Proliferation by Interacting with c-Myc. Journal of Biological Chemistry, 283(35), 23567-23580.

Yamada, O., Ozaki, K., Akiyama, M., \& Kawauchi, K. (2012). JAK-STAT and JAKPI3K-mTORC1 pathways regulate telomerase transcriptionally and posttranslationally in ATL cells. Molecular Cancer Therapeutics, 11(5), 1112-1121.

Yang, P., Yin, P., Tseng, L., Yang, C., Hsu, C., Lee, M., \& Chi, C. (2011). Rab5A is associated with axillary lymph node metastasis in breast cancer patients. Cancer Science, 102(12), 2172-2178.

Yoon, A., Gao, R., Kaul, Z., Choi, I.-K., Ryu, J., Noble, J. R., \& Wadhwa, R. (2011). MicroRNA-296 is enriched in cancer cells and downregulates p21WAF1 mRNA expression via interaction with its 3' untranslated region. Nucleic Acids Research, 39(18), 8078-8091.

Yu, Q., Liu, Y., Wang, C., Sun, D., Yang, X., Liu, Y., \& Liu, J. (2012). Chiral ruthenium(II) polypyridyl complexes: stabilization of g-quadruplex DNA, inhibition of telomerase activity and cellular uptake. Plos One, 7(12), e50902.

Yu, S. T., Li, C., Lü, M. H., Liang, G. P., Li, N., Tang, X. D., \& Yang, S. M. (2011). Noninvasive and real-time monitoring of the therapeutic response of tumors in vivo with an optimized hTERT promoter. Cancer, 118(7), 1884-1893.

Zech, T., \& Machesky, L. (2008). Rab5 and rac team up in cell motility. Cell, 134(1), 1820.

Zhang, B., Chen, J., Cheng, A. S. L., \& Ko, B. C. B. (2014). Depletion of sirtuin 1 (SIRT1) leads to epigenetic modifications of telomerase (TERT) gene in hepatocellular carcinoma cells. Plos One, 9(1), e84931.

Zhang, Y., Chen, L., Yang, S., \& Fang, D. (2012). E2F1: A potential negative regulator of hTERT transcription in normal cells upon activation of oncogenic c-Myc. Medical Science Monitor, 18(1), RA12-RA15.

Zhang, Y., Toh, L., Lau, P., \& Wang, X. (2012). Human telomerase reverse transcriptase (hTERT) is a novel target of the Wnt/ $\beta$-catenin pathway in human cancer. The Journal of Biological Chemistry, 287(39), 32494-32511.

Zhao, Y., Wang, S., Popova, E. Y., Grigoryev, S. A., \& Zhu, J. (2009). Rearrangement of upstrream sequences of the hTERT gene during cellular immortalization. Genes Chromosomes Cancer, 48(11), 963-974. 
Zhao, Z., Liu, X., Wu, H., Zou, S., Wang, J., Ni, P., \& Fan, Q. (2010). Rab5a overexpression promoting ovarian cancer cell proliferation may be associated with APPL1-related epidermal growth factor signaling pathway. Cancer Science, 101(6), 1454-1462.

Zhou, C., Steplowski, T. A., Dickens, H. K., Malloy, K. M., Gehrig, P. A., Boggess, J. F., \& Bae-Jump, V. L. (2013). Estrogen Induction of Telomerase Activity through Regulation of the Mitogen-Activated Protein Kinase (MAPK) Dependent Pathway in Human Endometrial Cancer Cells. PLoS ONE, 8(2), 1-10. 

Akt
c-Abl
c-Myc
E2
EGF
EGFR
ER
ERK
ETS2
GAP
GEF
hTERT
hTR
IGF-1
IGF-1R
MAPK
MCF-12A
$\mathrm{MCF} 7$
MDA-MB 231
MEK
miRNA
PI3K
Rab5
Protein kinase B; enzyme activated by PI3K and involved in a signal transduction pathway responsible for promoting cell growth, proliferation and survival.
Tyrosine kinase involved in various aspects of cell cycle regulation.
Transcription factor involved in promoting cell growth and proliferation.
Estradiol; hormone important in promoting cell proliferation.
Epidermal growth factor; growth factor that stimulates cell proliferation.
Epidermal growth factor receptor; binds EGF and can activate the Ras/MAPK pathway.
Estrogen receptor; binds estradiol.
Protein kinase of the MAPK pathway that is directly activated by MEK and translocates into the nucleus to activate various transcription factors.
Transcription factor important in promoting telomerase gene expression.
GTPase activating protein; protein that promotes the hydrolysis of GTP on small, monomeric G-proteins
Guanine nucleotide exchange factor; protein that promotes the exchange of GDP for GTP on small, monomeric G-proteins. Human telomerase reverse transcriptase; enzyme responsible for extending telomeres in certain cell types during cell division.
Human telomerase RNA; RNA template that binds to hTERT and is necessary for its function.
Insulin-like growth factor I; growth factor that stimulates cell proliferation.
Insulin-like growth factor I receptor; binds IGF-1 and can activate the Ras/MAPK pathway.
Mitogen activated protein kinase pathway; signaling pathway activated by Ras in response to growth factor stimulation.
Normal breast epithelial cell line.
Tumorigenic, non-invasive breast cancer cell line.
Tumorigenic, invasive breast cancer cell line.
Protein kinase of the MAPK pathway that is directly activated by RAF.
microRNA; small RNA molecule involved in post-transcriptional downregulation of gene expression.
Phosphoinositide 3-kinase; enzyme involved a signal transduction pathway responsible for promoting cell growth, proliferation and survival.
Small, monomeric GTPase activated by Rin 1 and involved in early endosome formation and vesicle trafficking.




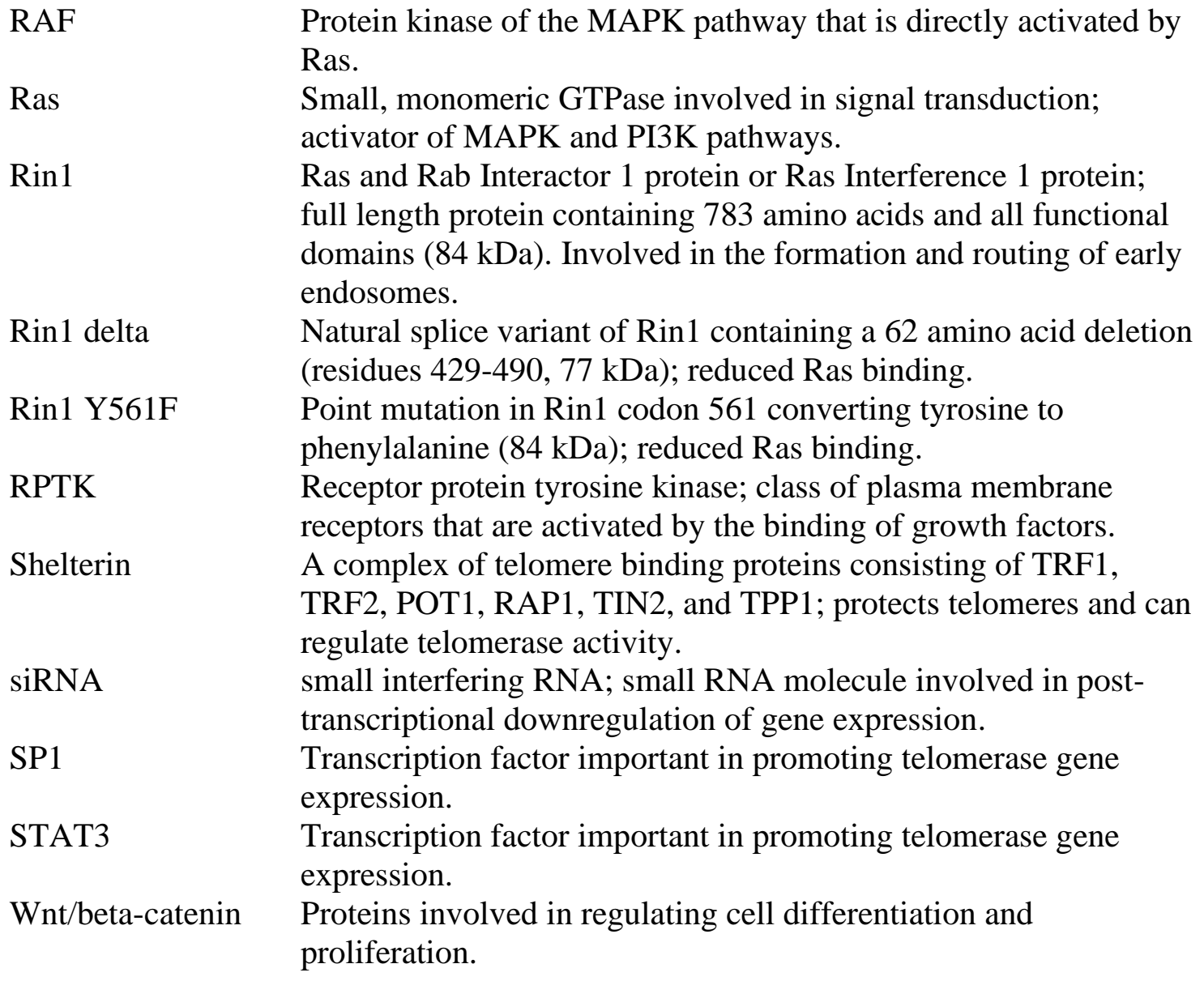

Table 1.1: Descriptions of key terms. 


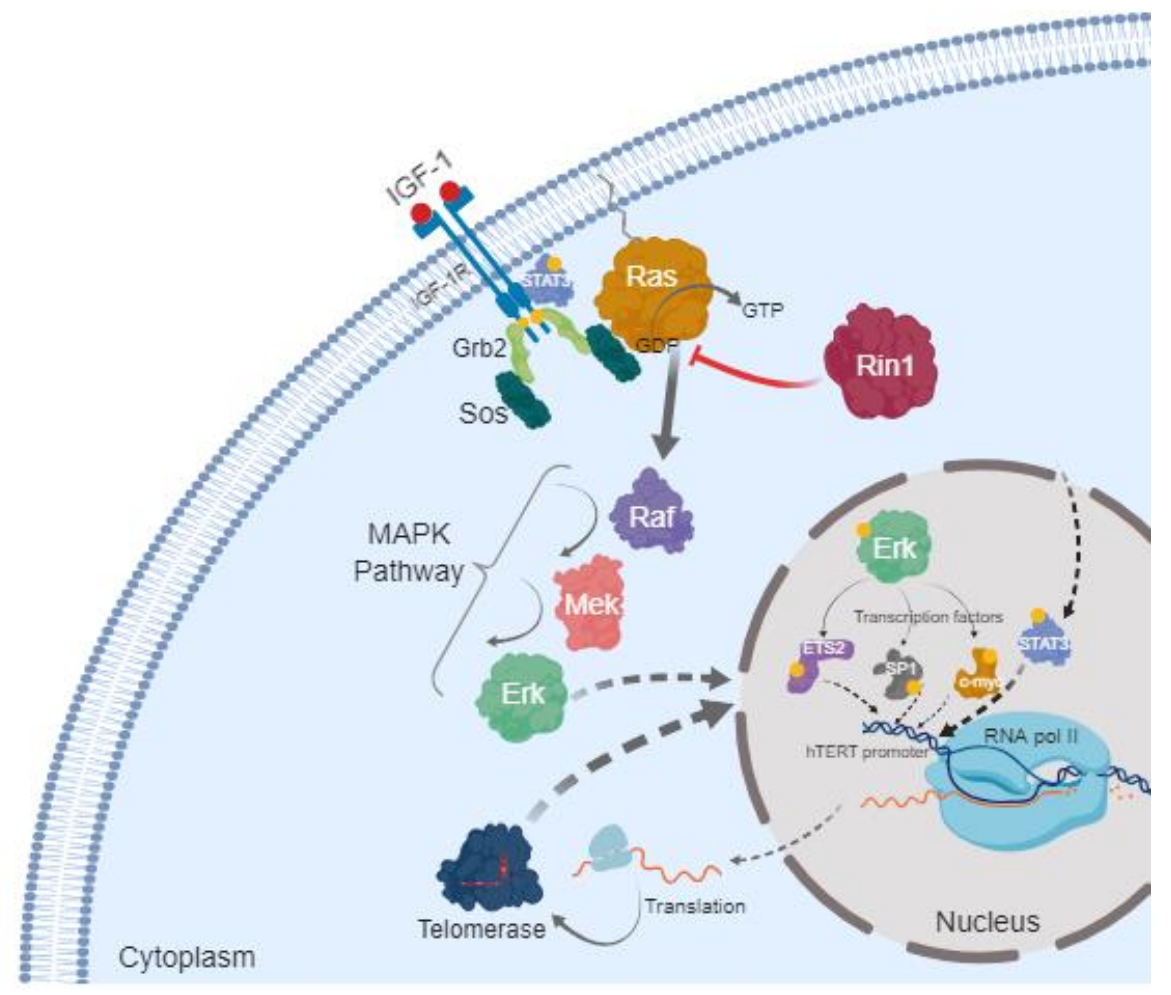

Figure 1.1: Diagram of the proposed model for the moderating influence of Rin1 on signaling through the Ras/MAPK pathway. The binding of a growth factor such as IGF1 to its receptor on the plasma membrane activates Ras and subsequently the MAPK pathway. Activation of the MAPK pathway in turn activates various nuclear transcription factors involved in telomerase expression. Rin1 competes with Raf for binding to activated Ras.

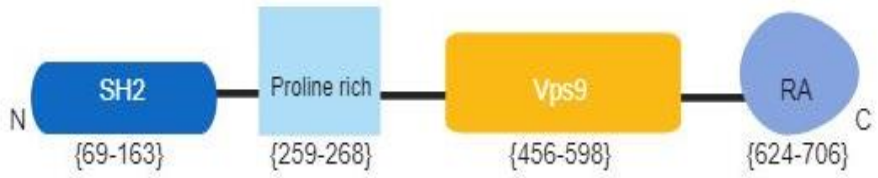

Figure 1.2: Rin1 domains. SH2 binds phospho-tyrosine residues. Vps9 acts as a guanine nucleotide exchange factor for Rab5. RA binds to activated Ras. Proline rich binds to SH3 domains. 


\section{CHAPTER 2}

\section{The Effect of Rin1 on Cellular Proliferation as well as on Telomerase Gene Expression and Activity in Breast Cancer Cells}

\subsection{Introduction}

Breast cancer is the most common cause of cancer in women and is the second most common cause of cancer-related mortality among women. Roughly $20 \%$ of all breast cancer cases are caused by triple negative breast cancers that are characterized by a loss of the estrogen receptor, progesterone receptor, and human epidermal growth factor receptor 2 (HER-2 or ERBB2) (Ayub et al., 2015). The significant tumorigenic role that growth factor receptors play in the development of many types of cancers has been widely reported, and it is especially true for breast cancer carcinogenesis. A large body of evidence supports the involvement of receptor protein tyrosine kinases (RPTKs), such as the insulinlike growth factor 1 receptor (IGF-1R) and the epidermal growth factor receptor (EGFR), in the development of many forms of breast cancer. Of equal importance, is the involvement of the steroid hormone estrogen receptor (ER) in tumorigenesis. The role of the estrogen receptor in the formation of breast cancer is also widely documented. In fact, there is a growing body of research that indicates that cooperativity between RPTKs and the estrogen receptor may exist and is essential for the development of the malignant phenotype.

One of the molecular hallmarks associated with invasive breast cancer is overexpression of the insulin-like growth factor 1 receptor. Upregulation of the IGF-1R and its ligand binding partners is one of the principal mechanisms by which oncogenesis occurs in breast epithelial cells. The IGF1-R is a hetero-tetrameric transmembrane protein that is 
structurally similar to the insulin receptor (IR). It is initially synthesized as a pro-receptor that is cleaved into $\alpha 2 \beta 2$ subunits before insertion into the cell membrane (Riedemann et al., 2007). When bound to the IGF-1 ligand, the receptor will undergo a conformational change to form a catalytically active dimer that is cross-phosphorylated on specific tyrosine residues located in the cytoplasmic C-terminal domains of the subunits (Verbeek et al., 1998). Stimulation of the EGF receptor by epidermal growth factor occurs in an almost identical manner.

Mitogenic signals are transduced to the interior of the cell via receptor mediated phosphorylation of adaptor proteins that bind the receptor phospho-tyrosine residues. The insulin receptor substrate 1 (IRS-1) and Src homology collagen (Shc) proteins typically associate with the activated IGF-1 receptor to become phosphorylated and then relay the growth signal to two primary intracellular signaling cascades: the PI3K/Akt pathway and the Ras/MAPK pathway (Mawson et al., 2004). Phosphatidyl inositol 3 kinase (PI3K) commonly binds to and is activated by phospho-IRS-1, and in turn will subsequently activate protein kinase B (Akt). Phosphorylated Shc will form a complex with the Grb2/SOS adaptor proteins to activate p21/Ras and ultimately the MAPK pathway. IGF-1 induction of the Ras/MAPK pathway leads to transcriptionally enhanced expression of genes that promote cellular proliferation while activation of the PI3K/Akt pathway is strongly related to cellular survival ( $\mathrm{Lu}$ et al., 2003). The authors also note that overexpression of the human epidermal growth factor 2 receptor in MCF-7 breast cancer cells can attenuate IGF-1 induced MAPK signaling by decreasing the levels of phosphorylated Shc. 
The reliance of most breast cancers on IGF-1 induced signaling for proliferation and survival is highlighted by the fact that the epidermal growth factor receptor cannot compensate for loss of IGF-1 signaling caused by the IGF-1R knockout. Riedemann et al. (2007) demonstrated that IGF-1R knockout in the non-invasive MCF-7 breast cancer cell line resulted in increased phosphorylation of EGFR and its effectors (JNK, extracellularsignal regulated kinases, STAT5), but that these cells were most sensitive to IGF-1R loss. Dual knockout of IGF-1R and EGFR resulted in the same level of clonogenic survival as IGF-1R knockout alone. Resistance to IGF-1R loss however can be conferred by the action of the insulin receptor (IR) given the high degree of homology between the two receptors, and the fact that IR levels are often elevated in many cancers. The IGF-1R and IR are highly selective in their affinities for their respective ligands, IGF-1 and insulin. However, IGF-II can bind to both the IGF-1R as well as a natural splice variant of the IR known as IR-A. Therefore, the IR can act as an additional receptor for the proliferative signaling of IGF-II even if IGF-1R levels are decreased (Ulanet et al., 2009; Malaguarnera and Belfiore, 2011).

The highly aggressive and invasive nature of most triple negative breast cancers and their resistance to traditional hormonal therapies/HER-2 inhibition has driven the need to discover selective small inhibitor molecules for IGF-1R. The approach has been difficult however given that many of these small inhibitors also interfere with function of the insulin receptor in normal tissues. Mukohara et al. (2009) have shown that the small IGF-1R inhibitor NVP-AEW541 was able to decrease levels of phosphorylated IGF-1R while also decreasing levels of phospho-Akt by disrupting the PI3K/IRS-1 complex. The effect of the inhibitor was strongest in an MCF-7 cell line that expressed high levels of IRS-1 but was 
less pronounced in the triple negative MDA-MB 231 cell line. On the other hand, Ayub et al. (2015) were able to demonstrate that dual inhibition of IGF-1R with NVP-AEW541 and another small inhibitor of either PI3K, mTORC, or MEK was able to reduce the phosphorylation of Akt and arrest the cell cycle at the G1 phase in MDA-MB 231 breast cancer cells.

The key strategy to finding effective treatments for breast cancers is to understand the molecular mechanisms that govern their proliferation, migration, and invasive behavior. Badache and Hynes (2001) report that Interleukin-6 may play a pivotal role in the migration of T47D breast cancer cells. An IL-6 treatment of T47D cells resulted in the activation of both the Ras/MAPK and PI3K/Akt pathways, and a subsequent increase in cell migration. The effect was abolished by treatment of cells with inhibitors of these pathways. The activation of the IL-6 receptor by IL-6 resulted in the recruitment and phosphorylation of the SHP-2 and Gab1 proteins- which in turn enhanced signaling through the MAPK and PI3K pathways. Interestingly, SHP-2 and Gab1 are also associated constitutively with the active EGFR and this may provide a cooperative link between the actions of these two receptors (Badache and Hynes, 2001). A similar study by Park et al. (2013) revealed that IL-32 $\beta$ stimulation of MDA-MB 231 breast cancer cells increased vascular endothelial growth factor (VEGF) secretion by these cells through activation of STAT3, the outcome of which was heightened migration and invasion.

Elevated migration and invasiveness of breast cancer cells is required for metastasis. Metastasis of breast cancer cells to lymph nodes is common and occurs primarily by VEGF-C induced lymph-angiogenesis. Zhu et al. (2011) treated MDA-MB 231 cells with varying concentrations of IGF-1 and noticed that VEGF-C secretion by these breast cancer 
cells increased in a dose dependent manner up to a concentration of $400 \mathrm{ng} / \mathrm{mL}$. The VEGFC secretion following IGF-1 stimulation was found to be mediated by both the PI3K/Akt and MAPK pathways. Inhibition of Akt abolished the IGF-1 induced expression of VEGFC while ERK inhibition reduced it. EGF induced migration and proliferation of MCF-7 cells has also been linked to sphingosine kinase I activation. Stimulation of these cells with EGF resulted in the activation and translocation of the enzyme to the plasma membrane where it phosphorylates membrane sphingolipids. Specific G protein-coupled receptors associated with the cell membrane then bind the phosphorylated sphingolipids and regulate cellular processes such as migration and proliferation (Sarkar et al., 2005). The Src family of kinases have also been implicated as important regulators of proliferation, migration, and invasiveness in MDA-MB 231 cells in response to growth factor stimulation of various RPTKs and cytokine receptors (Sanchez-Bailon et al., 2012). Additionally, exposure to the heavy metal cadmium (Cd) has also been shown to stimulate MAPK signaling and proliferation in MCF-7 cells (Song et al., 2015).

The migratory behavior of metastatic breast cancer cells is often driven by chemotaxis towards growth factors and changes in the extracellular matrix (ECM). Price et al. (1999) investigated the chemotaxis of MDA-MB 231 cells towards varying concentrations of EGF in the environment of the cells. The directional motility of the cells toward EGF was highest at an EGF concentration of $10 \mathrm{ng} / \mathrm{mL}$, and the chemotactic response could be inhibited to baseline levels through the addition of specific EGFR inhibitors. The attachment of cells to the extracellular matrix often occurs within areas known as focal adhesions. These regions consist of large protein complexes in which the cytoskeleton of the cell, through transmembrane proteins such as integrins, connects to the extracellular matrix. These focal 
adhesions are critical for the transmission of mechanical forces and regulatory signals between the cell and the ECM. A study by Taliaferro-Smith et al. (2015) revealed that the invasiveness of MDA-MB 231 breast cancer cells is related to the activity of the cytoplasmic focal adhesion kinase (FAK) enzyme. Inhibition of FAK by siRNA treatment resulted in decreased levels of total and active IFG-1R as well as reduced invasiveness.

Metastasis of cancer cells is often preceded by a series of alterations to both cellular morphology and the ECM known as the epithelial to mesenchymal transition (EMT). The epithelial to mesenchymal transition is typically characterized by enhanced cell migration and reduced cell adhesion to the ECM. Walsh and Damjanovski (2011) studied the role that transforming growth factor- $\beta$ (TGF- $\beta$ ) plays in mediating the epithelial to mesenchymal transition in MCF-7 breast cancer cells. The TGF- $\beta$ has been implicated in tumorigenesis given its ability to regulate cellular proliferation and differentiation. MCF7 cells were treated with $100 \mathrm{nM}$ of IGF-1 and then assayed for metalloproteinase activity. A 2.9 fold increase in metalloproteinase activity was observed when compared to control cells, and treatment with either MAPK or PI3K inhibitors could reduce this activity by as much as $29 \%$ to $34 \%$ respectively. When treated with TGF- $\beta$ immediately following IGF1 stimulation, the cells began to assume a more fibroblast-like morphology usually associated with the EMT.

The ability of cancer cells to become mobile and invade new tissues is largely dependent upon morphological changes mediated by rearrangement of the actin cytoskeleton. Mezi et al. (2012) report that cortactin is necessary in the regulation of membrane dynamics and for the formation of membrane ruffles and lamellipodia, structures frequently associated with cell migration. The MDA-MB 231 and MCF-7 cells exhibited increased membrane 
ruffling and lamellipodia formation following stimulation with either EGF or IGF-1. Phosphorylation of cortactin by Src and its localization to focal adhesion regions of the plasma membrane was observed to be time dependent in both breast cancer cell lines.

Additionally, Morimura and Takahashi (2011) report that stathmin and the small GTPase Rac1 may also help mediate invasiveness in MDA-MB 231 breast cancer cells. The metastatic potential of MCF-7 cells appears to be regulated to an extent by a transmembrane glycoprotein known as Mucin 1 (MUC1). The MUC1 is frequently overexpressed in these cells but with reduced levels of glycosylation, and it is abnormally located on the basal side of the cell membrane in close proximity to the ECM. In this position, aberrant MUC1 is then able to interact with an ECM protein known as ICAM-1. The interaction facilitates the attachment of migratory cancer cells to blood vessel walls and stimulates Src activation (Liao et al., 2014). IGF-1, EGF, and estradiol (E2) have also been shown to promote MCF-7 breast cancer cell attachment to the EMC glycoprotein fibronectin. High levels of fibronectin in the EMC have been correlated with increased migration and invasiveness in these cells (Voudouri et al., 2016).

The metastasis of breast cancer cells to other sites is commonly driven by chemotaxis towards locations that will provide a suitable environment for new tumor formation. A study by Helbig et al. (2003) reveals that NF-kB enhances expression of the chemokine receptor CXCR4. A ligand for this receptor, stromal derived factor- $1 \alpha$, is abundantly expressed at common metastatic sites and is a powerful attractant for migratory MDA-MB 231 breast cancer cells. Osseous tissue is a typical metastatic site for MDA-MB 231 cells as it provides a conducive environment for colonization and cellular proliferation. Once in bone tissue, these cells begin to secrete various factors such as parathyroid hormone-related 
peptide that in turn stimulate osteoclast activity. Increased bone resorption by osteoclasts results in an elevated release of growth factors, such as TGF- $\beta$ and IGFs, from the bone matrix. A harmful cycle of cancer cell proliferation and bone destruction then ensues (Son et al., 2010)

There is a growing body of evidence to support the idea that the development of breast cancer may involve the cooperative action of growth factors, such as EGF or IGF-1, and estrogen. When dually stimulated by both IGF-1 and estrogen, Mawson et al. (2004) observed that MCF-7 breast cancer cells quickly progressed into the S phase of the cell cycle. The progression was accompanied by an increase in the levels of c-Myc and cyclin D1. Estrogen induced the expression of both c-Myc and cyclin D1 while IGF-1 primarily induced the expression of cyclin D1 only. Thus, the differential regulation of c-Myc and cyclin D1 expression by estrogen and IGF-1 may represent a point of cooperativity in the regulation of breast cancer cell proliferation (Mawson et al., 2004).

A 2009 study by Santen et al. sheds further light on the cooperative effect of IGF-1R and estrogen stimulation on MAPK activity in MCF-7 cells. Estradiol (E2) stimulation of these cells resulted in a rapid phosphorylation of both IGF-1R and EGFR. Activation of IGF-1R consequently stimulated matrix metalloproteinase activity, and in turn the release of heparin-binding EGF (Hb-EGF) from its attachment to the plasma membrane. The subsequent binding of Hb-EGF to EGFR was then observed to increase MAPK activation and cell proliferation. Similarly, Fox et al. (2009) report that STAT5 is phosphorylated in the cytoplasm in response to E2 or EGF stimulation and then translocates to the nucleus where it regulates transcription of multiple proliferative genes. The proliferative response to E2 can be abolished by inhibition of either EGFR, Src, or STAT5. 
The importance of the estrogen receptor (ER) in the development of breast cancer is underscored by the fact the roughly $70 \%$ of all breast cancer cases exhibit abnormally high levels of ER. Estrogen exerts its proliferative effect by interacting with two predominantly nuclear receptors (ER $\alpha$ and ER $\beta$ ) as well as with a newly discovered G protein-coupled receptor known as GPR30. ER $\alpha$, because of its increased stability and availability, appears to play a greater role in the proliferative response of breast cancer cells to estrogen than does ER $\beta$ (Tecalco-Cruz and Ramirez-Jarquin, 2016). Zhang et al. (2014) for instance found that ERa36 is closely associated with the plasma membrane and is capable of inducing rapid MAPK signaling in MCF-7 cells through the involvement of several proteins including matrix metalloproteinases and Hb-EFG.

The expression of ER $\beta$, on the other hand, tends to be lower in breast cancer cells than in normal breast tissue. Lazennec et al. (2001) were able to demonstrate that overexpression of ER $\beta$ in MDA-MB 231 cells could reduce ER stimulated proliferation and migration by as much as $40 \%$ when compared to control. Besides the estrogen receptor, the G proteincoupled receptor GPR30 has also recently been shown to mediate estrogen induced signaling. Prakash-Pandey et al. (2009) report that connective tissue growth factor (CTGF) expression is strongly induced by $\mathrm{E} 2$ stimulation of $\mathrm{SKBr} 3$ breast cancer cells that contain GPR30 but lack both ER $\alpha$ and ER $\beta$. A two-fold increase in migration was subsequently observed following E2 stimulation and this effect could be greatly diminished by either GPR30 or CTGF inhibition.

In summary, the important role that growth factor receptors play in breast cancer tumorigenesis is clear. Abnormalities in these receptors, as well as the estrogen receptor, are often significant contributors to the acquisition of the cancerous phenotype- as are 
abnormalities in the signaling pathways that they regulate. The enhanced cell proliferation and migration commonly observed in most breast cancers is therefore intimately tied to the activity of mitogenic signaling pathways and their associated receptors.

\section{$2.2 \quad$ Results}

Lentiviral vectors encoding GFP and Rin1 were designed using the VectorBuilder online platform (https://en.vectorbuilder.com/). These lentiviral vectors (Figure 2.1) were used in the creation of the Rin1 and GFP expressing MDA-MB 231 and MCF-7 breast cancer cell lines as described in 2.4, Creation of Cell Lines. Additionally, MCF-12A normal breast epithelial cell lines expressing GFP and Rin1 were also created in the same manner. Confirmation of stable Rin1 and GFP expression was accomplished by Western blotting and immunofluorescence (IF). Western blotting (Figure 2.3) verified the expression of GFP and Rin1 in the respective MDA-MB 231 cell lines. A light micrograph image (Figure 2.2, B.) of the MDA-MB 231 Rin1 cell line reveals a rounded, clumped morphology and a generally slower growth pattern for these cells. A confocal micrograph image (Figure 2.2, A.) indicates the presence of Rin1 in the Rin1 virally transduced MDAMB 231 cell line. The spatial distribution of Rin1 throughout the MDA-MB 231 Rin1 cells is also evident from the confocal micrograph image (Figure 2.2, A.). Rin1 appears to be more concentrated near the plasma membrane.

A baseline experiment to assess the proliferative response of MDA-MB 231 cells to culture media containing serum (10\% FBS) was conducted to establish the normal proliferative capacity of unaltered, non-virally transduced MDA-MB 231 breast cancer cells (Figure 2.4). Three different concentrations of $5.0 \times 10^{4}, 1.0 \times 10^{5}$, and $2.0 \times 10^{5}$ cells $/ \mathrm{mL}$ were utilized, and each experiment was repeated in triplicate. When exposed to 
serum, all three cell concentrations resulted in an increase in proliferation as measured by the MTT assay. However, only concentrations of $1.0 \times 10^{5}$ and $2.0 \times 10^{5}$ cells $/ \mathrm{mL}$ displayed a statistically significant increase in cellular proliferation when compared to a corresponding serum free control group $(\mathrm{p}<0.05$, Student's t-test). A $22 \%$ increase in proliferation over the serum free treatment group was observed for a cell concentration of $5.0 \times 10^{4}$ cells $/ \mathrm{mL}$, while concentrations of $1.0 \times 10^{5}$ and $2.0 \times 10^{5}$ cells $/ \mathrm{mL}$ resulted in increases of approximately $40 \%$ and $83 \%$. These results demonstrate the effect of cell concentration on serum induced cellular proliferation in the MDA-MB 231cell line. Regression analysis (Figure 2.5) shows that the linear increase in cellular proliferation as a function of cell concentration is statistically significant for the serum treated groups ( $p$ $<0.05)$.

The MDA-MB 231 cells expressing Rin1 displayed reduced levels of cellular proliferation across a range of IGF-1 concentrations tested when compared to control cells expressing GFP (Figure 2.6). The MDA-MB 231 GFP cells exhibited increases in cellular proliferation over unstimulated cells ranging from $47 \%$ for treatment with $20 \mathrm{ng} / \mathrm{mL}$ of IGF-1 to $80 \%$ for treatment with $5 \mathrm{ng} / \mathrm{mL}$ of IGF-1. Conversely, the range of increase in cellular proliferation for the MDA-MB 231 Rin1 cells was much lower- ranging from $6 \%$ for treatment with $100 \mathrm{ng} / \mathrm{mL}$ of IGF-1 to only $29 \%$ for treatment with $20 \mathrm{ng} / \mathrm{mL}$ of IGF1. These data suggest a suppressive role of Rin1 on cellular proliferation following IGF-1 stimulation.

In an effort to validate these findings, additional experimentation to determine the effect of Rin1 expression on cellular proliferation in MDA-MB 231, MCF-12A, and MCF7 cells was performed by stimulating cells with an IGF-1 concentration of $100 \mathrm{ng} / \mathrm{mL}$ for 24 hours. 
IGF-1 stimulation resulted in a significant increase in proliferation of $56 \%$ over serum starved cells for the MDA-MB 231 non-virally transduced cell line (Figure 2.7). Similarly, a significant increase in cell proliferation of $65 \%$ in response to IGF-1 exposure was noted for the MDA-MB 231 GFP cell line (Figure 2.8). However, the MDA-MB 231 Rin1 expressing cell line displayed a significant increase in cellular proliferation of only $18 \%$ when compared to the corresponding serum free group (Figure 2.9). The serum starved condition resulted in roughly equal low levels of proliferation for all three MDA-MB 231 cell lines examined and was set equal to 1 for analysis purposes. All experiments were conducted in triplicate using a concentration of $2.0 \times 10^{5}$ cells $/ \mathrm{mL}$.

In terms of cell viability, all three MDA-MB 231 cell lines tested showed no significant increases in cell viability in response to IGF-1 stimulation when compared to the corresponding serum free groups. However, similar to cellular proliferation, the MDA-MB 231 Rin1 expressing cell line exhibited the lowest level of cell viability (24\%) when stimulated by IGF-1. All viability experiments were conducted in triplicate using a concentration of $2.0 \times 10^{5}$ cells $/ \mathrm{mL}$.

A serial dilution of a known telomerase positive HeLa cell extract was performed in order to demonstrate the ability of the telomeric repeat amplification protocol (TRAP) qPCR assay to accurately measure telomerase activity in diluted cell extract samples with different concentrations of telomerase (Figure 2.11). As the dilution factor increased by factors of 10 from $10 \mathrm{X}$ to $100 \mathrm{X}$ to $1,000 \mathrm{X}$, the average cycle threshold $(\mathrm{Ct})$ value increased from 26.28 for the undiluted sample to 26.78 for the 1,000X diluted sample. The $10 \mathrm{X}$ and 100X dilutions produced $\mathrm{Ct}$ values of 26.50 and 26.70. The highest $\mathrm{Ct}$ value of 27.02 was observed for the buffer only sample containing no cell extract any only the assay reagents 
(Table 2.1). The PAGE gel analysis for the undiluted telomerase positive sample reveals the characteristic ladder of DNA bands consistent with the presence of telomerase activity (Figure 2.10). The assay for each dilution factor was performed in duplicate.

A reverse transcriptase qPCR (RT-qPCR) assay was performed to assess the effect of IGF-1 stimulation (100 ng/mL for 24 hours) on telomerase gene expression in the MDAMB 231 Rin1 and GFP cell lines (Figure 2.13). Telomerase gene expression for all cell samples was normalized to that of the GAPDH housekeeping gene using the $\Delta \mathrm{Ct}$ method. A normalized average telomerase gene expression Ct value of 10.87 in response to IGF-1 stimulation was found for the MDA-MB 231 cells overexpressing Rin1 (Table 2.3). The corresponding IGF-1 stimulated GFP control cells displayed a normalized average telomerase gene expression $\mathrm{Ct}$ value of 10.74 (Table 2.3). A 1.09 fold decrease in telomerase gene expression was observed for the cells overexpressing Rin1 when compared to the GFP expressing cells (Table 2.3). The fold change in telomerase gene expression was calculated using the $\Delta \Delta \mathrm{Ct}$ method where fold change is equal to $2^{-\Delta \Delta \mathrm{Ct}}$. The $\Delta \mathrm{Ct}$ is the difference between the average sample $\mathrm{Ct}$ for a gene of interest and that of a reference or housekeeping gene. The $\Delta \Delta \mathrm{Ct}$, therefore, is the difference in $\Delta \mathrm{Ct}$ values for a sample of interest and that of a particular reference sample. All samples were assayed in duplicate. The presence and specific amplification of GAPDH and telomerase qPCR products was confirmed through PAGE gel analysis (Figure 2.13) and melt curve analysis. A qPCR telomerase activity assay (as described in 2.4, Telomerase Activity Assay) was performed to determine the effect of Rin1 on telomerase activity following IGF-1 stimulation with $100 \mathrm{ng} / \mathrm{mL}$ for 24 hours (Table 2.2). Telomerase activity was assessed in both the MDA-MB 231 Rin1 and GFP cell lines as well as in telomerase positive and 
negative controls. The MDA-MB 231 cell samples expressing Rin1 resulted in an average Ct value of 21.14 following IGF-1 stimulation while the average Ct value of the corresponding GFP cell samples was 20.49 (Table 2.2). A 1.56 fold decrease in telomerase activity was observed for the cells overexpressing Rin1 when compared to the activity of the reference GFP cells (Table 2.2). Fold change was calculated according to the $\Delta \Delta \mathrm{Ct}$ method. The lowest average $\mathrm{Ct}$ value was observed for the HeLa cell telomerase positive control while the heat treated negative control and buffer only samples produced average Ct values of 20.16 and 20.23 respectively (Table 2.2). The qPCR amplification of the TRAP products was confirmed by PAGE gel analysis (Figure 2.12). All samples were assayed in duplicate.

Cellular proliferation and viability as well as telomerase activity of normal, nontumorigenic MCF-12A breast epithelial cells were also assayed following IGF-1 exposure. IGF-1 stimulation of non-transduced MCF-12A cells with $100 \mathrm{ng} / \mathrm{mL}$ for 24 hours resulted in an insignificant decrease in cellular proliferation of 20\% over serum starved cells (Figure 2.15). On the other hand, an insignificant increase in cell proliferation of $41 \%$ in response to IGF-1 exposure was noted for the MCF-12A Rin1 cell line (Figure 2.17). The MCF-12A GFP expressing cells displayed an insignificant increase in proliferation following IGF-1 exposure of $28 \%$ when compared to the corresponding serum free group (Figure 2.16). The serum starved condition resulted in roughly equal low levels of cellular proliferation for all three MCF-12A cell lines examined and this value was set equal to 1 for analysis purposes. All cellular proliferation experiments were conducted in triplicate using a concentration of $2.0 \times 10^{5}$ cells $/ \mathrm{mL}$. 
All three MCF-12A cell lines tested showed no significant increases in cell viability in response to IGF-1 stimulation when compared to the corresponding serum free groups. However, when compared as a whole, the MCF-12A Rin1 cell line exhibited the lowest level of cell viability (11\%) when stimulated by IGF-1. All cell viability experiments were conducted in triplicate using a concentration of $2.0 \times 10^{5}$ cells $/ \mathrm{mL}$.

To assess telomerase activity, MCF-12A cells expressing Rin1 or GFP were serum starved for 24 hours and then treated with $100 \mathrm{ng} / \mathrm{mL}$ of IGF-1 for an additional 24 hours. The IGF-1 treated cell samples and corresponding untreated samples were then lysed and prepared for the TRAP qPCR telomerase activity assay as described in 2.4, Telomerase Activity Assay. The MCF-12A cell samples expressing Rin1 resulted in an average Ct value of 25.76 following IGF-1 stimulation while the average $\mathrm{Ct}$ value of the corresponding GFP cell samples was 25.92 (Table 2.4). A 1.1 fold increase in telomerase activity was observed for the cells overexpressing Rin1 when compared to the activity of the control GFP cells (Table 2.4). Fold change was calculated according to the $\Delta \Delta \mathrm{Ct}$ method. The heat treated negative control and buffer only samples produced average $\mathrm{Ct}$ values of 25.70 and 26.06 respectively (Table 2.4). All samples were assayed in duplicate.

A reverse transcriptase qPCR (RT-qPCR) assay was performed to assess the effect of IGF-1 stimulation (100 ng/mL for 24 hours) on telomerase gene expression in the MCF12A Rin1 and GFP cell lines (Table 2.5). Telomerase gene expression for all cell samples was normalized to that of the GAPDH housekeeping gene using the $\Delta \mathrm{Ct}$ method. A normalized telomerase gene expression $\mathrm{Ct}$ value of 5.58 in response to IGF-1 stimulation was observed for the MCF-12A cells overexpressing Rin1 (Table 2.5). The corresponding IGF-1 stimulated GFP control cells displayed a normalized telomerase gene expression Ct 
value of 4.23 (Table 2.5). A 2.55 fold decrease in telomerase gene expression was observed for the cells overexpressing Rin1 when compared to the GFP expressing cells (Table 2.5).

Finally, the effect of Rin1 on cellular proliferation and viability as well as the effect on telomerase gene expression and activity was investigated in the tumorigenic, non-invasive MCF7 human breast cancer cell line. In the non-virally transduced MCF7 cells, a significant increase of $26 \%$ in proliferation was observed in response to IGF-1 exposure when compared to the corresponding serum starved cells (Figure 2.19). The trend was also observed for the MCF7 cells expressing either GFP, 22\% increase, or Rin1, 26\% increase (Figures 2.20 and 2.21). Like the MDA-MB 231 and MCF-12A cells, the MCF7 cells expressing Rin1 exhibited the lowest level of viability following serum starvation for all three MCF7 cell lines examined.

A reverse transcriptase qPCR (RT-qPCR) assay was performed to assess the effect of IGF-1 stimulation (100 ng/mL for 24 hours) on telomerase gene expression in the MCF-7 Rin1 and GFP cell lines (Table 2.7). Telomerase gene expression for all cell samples was normalized to that of the GAPDH housekeeping gene using the $\Delta \mathrm{Ct}$ method. A normalized telomerase gene expression $\mathrm{Ct}$ value of 10.87 in response to IGF-1 stimulation was found for the MCF7 cells overexpressing Rin1 (Table 2.7). The corresponding IGF-1 stimulated GFP control cells displayed a normalized telomerase gene expression Ct value of 10.54 (Table 2.7). A 1.26 fold decrease in telomerase gene expression was observed for the cells overexpressing Rin1 when compared to the GFP expressing cells (Table 2.7).

To assess telomerase activity, MCF7 cells expressing Rin1 or GFP were serum starved for 24 hours and then treated with $100 \mathrm{ng} / \mathrm{mL}$ of IGF-1 for an additional 24 hours. The IGF-1 treated cell samples and corresponding untreated samples were then lysed and 
prepared for the TRAP qPCR telomerase activity assay as described in 2.4, Telomerase Activity Assay. The MCF7 cell samples expressing Rin1 resulted in an average $\mathrm{Ct}$ value of 24.63 following IGF-1 stimulation, while the average $\mathrm{Ct}$ value of the corresponding GFP cell samples was 24.50 (Table 2.6). A 1.09 fold decrease in telomerase activity was observed for the cells overexpressing Rin1 when compared to the activity of the control GFP cells (Table 2.6). Fold change was calculated according to the $\Delta \Delta \mathrm{Ct}$ method. The heat treated negative control and buffer only samples produced average values of 25.49 and 25.70 respectively (Table 2.6). All samples for telomerase gene expression and activity were assayed in duplicate.

\subsection{Discussion}

The MDA-MB 231 breast cancer cell line was chosen to study the effect of IGF-1 induction on cellular proliferation given the strong proliferative and invasive properties of this particular cell line. Two MDA-MB 231 control cell lines were used to compare the effects of Rin1 overexpression on proliferation and viability. These control cell lines consisted of virally unaltered (non-transduced) MDA-MB 231 cells as well as cells virally altered to express GFP.

As expected, a linear increase in cellular proliferation was observed following the exposure of non-transduced MDA-MB 231 cells to serum containing culture media (Figure 2.5). The increase in proliferation was significantly greater at higher cell concentrations $\left(1.0 \times 10^{5}\right.$ and $2.0 \times 10^{5}$ cells $\left./ \mathrm{mL}\right)$ when compared to the corresponding serum free treatment cells. Additionally, the linear increase in proliferation as cell concentration increased was also statistically significant for the serum treated cells $(\mathrm{p}<0.05)$ (Figure 2.5). These baseline results indicated that MDA-MB 231 cells were capable of proliferation in 
response to serum-containing growth factor stimulation. A cell concentration of $2.0 \times 10^{5}$ cells $/ \mathrm{mL}$ resulted in an $83 \%$ increase in cell proliferation over the serum free treatment and was therefore deemed to be an optimal concentration to use for future experimentation on cellular proliferation and viability.

Western blot analysis of virally transduced MDA-MB 231 cells indicates high levels of GFP as well as Rin1 expression (Figure 2.3). Light micrograph imagery of the Rin1 overexpressing MDA-MB 231 cell line reveals a striking difference in morphology and growth pattern. The Rin 1 cells typically grow more slowly and in a clumped or island-like pattern when compared to the GFP cell line and are more rounded in shape (Figure 2.2, B.). The GFP cells, which express endogenous levels of Rin1, tend to grow more evenly across the surface of the culture dish and have a more spindle-like appearance.

The reduced growth rate observed for the Rin1 overexpression cells could be a consequence of the strong ability for Rin1 to compete for access to activated Ras and thus attenuate signaling through the MAPK pathway. Confocal micrograph imagery reveals a spatial difference in the distribution of Rin1 as well. Rin1 appears to be more concentrated around the periphery of the cell in association with the plasma membrane, perhaps on endocytic vesicles (Figure 2.2, A.).

All three MDA-MB 231 cell lines displayed a significant increase in cell proliferation over the corresponding serum free condition (Figures 2.7, 2.8, and 2.9) following exposure to IGF-1. As expected, all three MDA-MB 231 cell lines also displayed roughly equal low levels of proliferation when serum starved and not exposed to IGF-1. However, in response to IGF-1 stimulation alone, the Rin1 expressing cells only exhibited a modest $18 \%$ increase in proliferation over the corresponding unstimulated cells (Figure 2.9). In fact, these cells 
displayed lower levels of cellular proliferation over a range of IGF-1 concentrations tested in comparison to GFP cells (Figure 2.6). The viability of these cells was also $43 \%$ lower in comparison to that of the non-transfected cells and $31 \%$ lower when compared to the GFP cells. Taken together, these results support the idea of Rin1 exerting a modulating effect on cellular proliferation and viability in MDA-MB 231 cells.

These results are in line with the results of the telomerase gene expression and activity studies and suggest that Rin1 may be able to moderate signaling through mitogenic pathways given its strong affinity for activated Ras. In order to assess the effect of Rin1 on telomerase gene expression following IGF-1 stimulation, MDA-MB 231 cells overexpressing Rin1 were compared to GFP expressing cells. RT-qPCR data reveal a 1.09 fold decrease in telomerase gene expression for the Rin1 cells after normalization to expression of the GAPDH housekeeping gene (Table 2.3). Successful amplification of both telomerase and GPADH is evident from the tight clustering of curves within the appropriate range of $\mathrm{Ct}$ values for each gene as well as the presence of specific bands of the correct amplicon sizes (Figure 2.13) and melting temperatures. The MDA-MB 231 cell lines contain a constitutively active mutant form of Ras, and thus the overexpression of Rin1 may be modulating signaling from this protein as evidenced in the reduction of telomerase gene expression by 1.09 fold (Table 2.3). Telomerase activity follows a similar trend as gene expression for the MDA-MB 231 Rin1 cells. Here, a 1.56 fold decrease in telomerase activity was observed for the Rin1 cells as compared to the GFP cells (Table 2.2). The decrease in telomerase activity is evident upon PAGE gel analysis of the TRAP qPCR products (Figure 2.12). The reduction in telomerase activity may be tied to the decrease in telomerase gene expression observed in the MDA-MB 231 Rin1 cells. 
Telomerase gene expression and activity in the normal, non-tumorigenic MCF-12A epithelial breast cell line appears to support a tumor suppressive role for Rin1 similar to that observed for the MDA-MB 231 breast cancer cell lines. Here, a 2.55 fold decrease in telomerase gene expression was recorded for the cells overexpressing Rin 1 when compared to control cells expressing GFP (Table 2.5). The decrease in telomerase gene expression is much larger than the 1.09 fold decrease observed in the MDA-MB 231 cells expressing Rin1. The greater decrease in telomerase gene expression exhibited by the MCF-12A cells may be attributed to the ability of Rin1 to modulate MAPK signaling more strongly in a normal, non-tumorigenic cell lines than in an invasive, tumorigenic cell line such as MDAMB 231 cells where telomerase expression and activity are strongly tied to cellular proliferation and survival.

The effect of Rin1 on telomerase activity however is not as clear for the MCF-12A cells. Generally, a tumor suppressor role is supported when comparing the telomerase activity of MCF-12A cells overexpressing Rin1 to non-virally transduced cells. Here, a 1.23 fold decrease in telomerase activity is observed for the cells expressing Rin1. The value is similar to the 1.56 fold decrease in activity reported for the MDA-MB 231 Rin1 cells. On the other hand, a 1.12 fold increase in telomerase activity is reported when comparing the MCF-12A Rin1 cells to the corresponding GFP cells (Table 2.4). The increase in telomerase activity exhibited by MCF-12A Rin1 cells also mirrors an increased level of cellular proliferation for these cells. Higher levels of proliferation were observed for MCF12A Rin1 cells following IGF-1 exposure (41\%) when compared to GFP cells. The downregulation of telomerase gene expression observed in these cells appears to be 
independent and in contrast to the recorded increased levels of telomerase activity and cellular proliferation.

The telomerase gene expression and activity data from the tumorigenic, non-invasive MCF7 breast cancer cell line lends further support to a potential tumor suppressor role for Rin1. Here, the 1.09 fold decrease in telomerase activity (Table 2.6) for cells expressing Rin1 over GFP cells is similar to that of the 1.56 fold decrease in telomerase activity observed for the MDA-MB 231 cells. A more interesting trend, however, is apparent when examining the ability of Rin1 to downregulate telomerase gene expression across the three different breast cell lines studied. The 1.26 fold reduction in telomerase gene expression observed for the MCF7 cells expressing Rin1 (Table 2.7) is between that of the MDA-MB 231 and MCF-12A cells, which displayed fold decreases each of 1.09 and 2.55 respectively. Rin1 therefore has the least ability to attenuate cell signaling following IGF1 stimulation, and thus lower telomerase gene expression, in the MDA-MB 231 cells. The result could be partially explained by the fact that these cells harbor a constitutively active mutant form of Ras and, as a consequence, the MDA-MB 231 cells are highly tumorigenic as well as invasive.

On the other hand, Rin1 has the greatest suppressive effect on telomerase gene expression in the normal, non-tumorigenic/non-invasive MCF-12A breast cells. Here, in the absence of a constitutively active form of Ras, Rin1 may have a stronger ability to dampen cell signaling subsequent to growth factor stimulation and therefore have a larger suppressive effect on telomerase gene expression. Finally, the ability of Rin1 to reduce telomerase gene expression in the tumorigenic, non-invasive MCF7 cells is intermediate (1.26 fold) between that of the MDA-MB 231 and MCF-12A cells. This result mirrors the 
$26 \%$ increase in cellular proliferation observed for these cells that falls between the $18 \%$ and $41 \%$ increases observed for the MDA-MB 231 Rin1 and MCF-12A Rin1 cells respectively. Again, as with the MDA-MB 231 cells, background tumorigenic processes in the MCF7 Rin1 cells may counteract the modulating influence of Rin1 on MPAK signaling.

In summary, Rin1 may act to attenuate signaling through the MAPK pathway given its strong interaction with activated Ras. This idea is supported by cellular proliferation data as well as by telomerase gene expression and activity studies from the MDA-MB 231, MCF7, and MCF12-A human breast cell lines. The tumor suppressive role of Rin1 in these breast cell lines may be cell type specific, with lower levels of cellular proliferation and a stronger reduction in telomerase activity being observed in the two tumorigenic cell lines versus the non-tumorigenic cell line (Table 2.8).

\subsection{Materials and Methods}

Characteristics of Cell Lines

The MDA-MB 231 breast cancer cell line is a highly invasive and tumorigenic breast epithelial (carcinoma) cell line that harbors a constitutively active mutant form of Ras, KRAS (G13D) (Kim et al., 2015). These cells are also triple negative meaning that they lack expression of the estrogen, progesterone, and HER2/Neu receptors and typically grow well in DMEM culture media supplemented with $10 \%$ fetal bovine serum. The MCF7 breast cancer cell line is a tumorigenic, non-invasive breast epithelial cell line that also grows well in DMEM culture media supplemented with 10\% FBS (Kim et al., 2015). The MCF-12A cell line is a normal breast epithelial cell line that grows optimally in a 1:1 
DMEM/F-12 culture media supplemented with 5\% horse serum, insulin, cholera toxin, EGF, and hydrocortisone (Kim et al., 2015).

\section{Creation of Cell Lines}

The MDA-MB 231 metastatic breast cancer cells were used to create cell lines expressing GFP or Rin1. Lentiviral plasmid expression vectors for GFP (pLV[Exp]-PuroCMV>eGFP) and Rin1 (pLV[Exp]-Puro-CMV>hRIN1) were designed using the VectorBuilder website (https://en.vectorbuilder.com/) powered by Cyagen Biosciences. The protein coding open reading frame sequence of each vector was placed under the control of a strong CMV promoter. A puromycin drug selection marker was included in each vector to allow for selection of stably transduced cells at a concentration of $4 \mathrm{ug} / \mathrm{mL}$. The GFP and various Rin1 lentiviral vectors were then synthesized by Cyagen Biosciences and each was accompanied with a maxiprep of plasmid vector DNA at a concentration of approximately $1 \mathrm{ug} / \mathrm{uL}$ (500 uL total volume).

A lentiviral expression system (Lenti-X Packaging Single Shots) available from Clontech Laboratories (Takara) was utilized to transduce native MDA-MB 231 cells to express GFP or Rin1. Transduction was performed according to the manufacturer's instructions. Briefly, $2.0 \times 10^{6}$ Lenti-X 293T viral packaging cells were plated on a $6 \mathrm{~cm}$ culture dish in $4 \mathrm{~mL}$ of complete growth media lacking tetracycline. The cells were plated 24 hours prior to transfection and allowed to grow at $37^{\circ} \mathrm{C}$ and $5 \% \mathrm{CO}_{2}$ until they reached approximately 80 to $90 \%$ confluency. On the day of transfection, 7 ug of plasmid DNA was mixed with sterile water to a final concentration of $600 \mathrm{uL}$. The entire volume of diluted DNA was then added to a single Lenti-X Packaging Single Shot tube and vortexed for roughly 20 seconds. The DNA sample was then allowed to incubate for 10 minutes. 
Following incubation, $300 \mathrm{uL}$ plasmid DNA sample was added to a culture dish containing the Lenti-X 293T viral packaging cells. The protocol was followed for each of the four lentiviral vector plasmid DNA samples and was performed in duplicate. The viral packaging cells were then incubated for 48 hours at $37^{\circ} \mathrm{C}$ and $5 \% \mathrm{CO}_{2}$. After 48 hours, the viral containing supernatant was collected and filtered through a 0.45 um filter to remove any cellular debris. Five hundred microliters of viral supernatant was then added to MDAMB 231 cells plated at a density of $3.0 \times 10^{5}$ cells per well on a 6 well plate in $2 \mathrm{~mL}$ of complete growth media.

The native MDA-MB 231 cells were plated one day prior to viral transduction. Polybrene was added to each well at a concentration of $6 \mathrm{ug} / \mathrm{mL}$ to enhance transduction. Transduction was performed in triplicate (3 wells) for each plasmid DNA sample. Any unused viral supernatant was aliquoted in $1.5 \mathrm{~mL}$ Eppendorf tubes and frozen at $-80{ }^{\circ} \mathrm{C}$. Viral transduction of the MDA-MB 231 cells occurred for 48 hours after which time the viral containing media was aspirated and replaced with $3 \mathrm{~mL}$ of fresh complete growth media. The cells were allowed to recover for an additional 48 hours prior to selection with puromycin. After the recovery period, the complete growth media was changed for $3 \mathrm{~mL}$ of selection growth media containing the antibiotic puromycin at a concentration of 4 ug/mL. The transduced MDA-MB 231 cells were selected for 72 hours before being trypsinized and moved to a T75 tissue culture flask. The cells were subsequently grown in selection media for a period of two weeks with media changes every three days until only stably transduced cells survived. The stable cell lines expressing GFP or Rin1 were then frozen in cryogenic tubes at $-80{ }^{\circ} \mathrm{C}$ until further use. 
Additionally, MCF-7 non-metastatic breast cancer cell lines and normal breast epithelial MCF-12A cell lines were created to stably express Rin1 or GFP following the methodology outlined above. Stable expression of Rin and GFP in these cell lines was achieved through selection using a puromycin concentration of $2 \mu \mathrm{g} / \mathrm{mL}$.

\section{MTT Cell Proliferation Assay}

An initial experiment to determine the optimal concentration of cells needed to observe a statistically significant difference in proliferation was performed using MDA-MB 231 breast cancer cells expressing green fluorescent protein (GFP). The cells were obtained from a cryopreserved stock culture and were grown in a T75 $\left(75 \mathrm{~cm}^{2}\right)$ tissue culture flask containing $15 \mathrm{~mL}$ of complete growth media incubated at $37^{\circ} \mathrm{C}$ with $5 \% \mathrm{CO}_{2}$. The cells were grown until approximately $80 \%$ confluent. The culture media consisted of $1 \mathrm{X}$ Dulbecco's Modified Eagle's Medium (DMEM) containing 4.5 g/L of glucose with Lglutamine. Complete culture media was prepared by filtering $500 \mathrm{~mL}$ of $1 \mathrm{X}$ DMEM supplemented with $10 \%$ fetal bovine serum (FBS), $1 \%$ penicillin-streptomycin $(10,000$ $\mathrm{U} / \mathrm{mL}$ penicillin and 10,000 ug/mL streptomycin), 1\% Minimal Essential Medium (MEM) nonessential amino acid solution, and $1 \%$ sodium-pyruvate $(100 \mathrm{mM})$. After reaching approximately $80 \%$ confluency, the culture medium was aspirated and the cells were washed once with $2 \mathrm{~mL}$ of cold $1 \mathrm{X}$ PBS-EDTA $(1 \mathrm{mM})$ solution. The cellular monolayer was then trypsinized in an incubator $\left(37^{\circ} \mathrm{C}\right.$ and $\left.5 \% \mathrm{CO}_{2}\right)$ to detach cells for 3 minutes with $2 \mathrm{~mL}$ of Trypsin-EDTA $(0.25 \%)$ solution. Four milliliters of serum free DMEM starvation medium was then added to the culture flask to suspend the trypsinized cells. The $4 \mathrm{~mL}$ cell suspension was then transferred to a $15 \mathrm{~mL}$ conical centrifuge tube and spun at 5,000 RPM in a centrifuge for three to five minutes until a cell pellet formed at the bottom of the tube. 
The entire volume of supernatant was then aspirated carefully so as to not disturb the pellet, and the pellet was re-suspended in a fresh volume of $2 \mathrm{~mL}$ of serum free starvation medium. A one hundred microliter sample of the re-suspended cells was then transferred to a $1 \mathrm{~mL}$ Eppendorf microcentrifuge tube. An equal volume of 100 uL of Trypan blue cell viability exclusion stain was added to the tube and gently mixed to permit detection of viable (live) cells. A $10 \mathrm{uL}$ sample of the stained cell suspension was then loaded into one chamber (side A) of a Bio-Rad dual chamber cell counting slide. A second $10 \mathrm{uL}$ sample was also loaded into the second chamber (Side B) and the total number of viable cells per milliliter in each chamber was counted using a Bio-Rad TC20 Automated Cell Counter. The average number of live cells per milliliter was then calculated and used to perform dilutions of the $2 \mathrm{~mL}$ suspension to specific cell concentrations in order to test the effect of serum versus serum free media on the proliferation of MDA-MB 231 eGFP cells. Serum free starvation media was used to prepare all dilutions.

Concentrations of $5.0 \times 10^{4}, 1.0 \times 10^{5}$, and $2.0 \times 10^{5}$ cells $/ \mathrm{mL}$ were tested initially to determine the optimal cell concentration needed to observe a meaningful, statistically significant difference in cellular proliferation as measured by an MTT assay. Each of the three concentrations tested were plated on separate 12 well plates ( 3 plates total, one for each cell concentration) in a volume of $1 \mathrm{~mL}$ per well $\left(5.0 \times 10^{4}\right.$ cells/well, $1.0 \times 10^{5}$ cells/well, $2.0 \times 10^{5}$ cells/well), and incubated under serum free conditions at $37^{\circ} \mathrm{C}$ with $5 \% \mathrm{CO}_{2}$ for 24 hours to synchronize growth rates. After 24 hours, the culture media in half of the wells (6) per plate was aspirated and replaced with $1 \mathrm{~mL}$ of complete growth media. The other half of the wells (6) retained the serum free starvation media. The plates were then incubated at $37^{\circ} \mathrm{C}$ with $5 \% \mathrm{CO}_{2}$ for another 24 hours. A $4 \mathrm{~mL}$ MTT (3-(4,5- 
dimethylthiazol-2-yl)-2,5-diphenyltetrazolium bromide) solution at a concentration of 5 $\mathrm{mg} / \mathrm{mL}$ was prepared, and $50 \mathrm{uL}$ was added to each well of each plate. The plates were gently agitated to ensure thorough mixing of the MTT solution with the liquid in each well. The plates were then wrapped in aluminum foil to prevent light exposure and were incubated at $37^{\circ} \mathrm{C}$ with $5 \% \mathrm{CO}_{2}$ for 4 hours to allow for reduction of the yellow tetrazolium dye. After 4 hours, the culture media in each well was carefully removed so as to not disturb the formazan crystals produced by the reduction of the tetrazolium dye. Four hundred microliters of dimethyl sulfoxide (DMSO) was then added to each well of each plate to dissolve the formazan crystals and the plates were agitated for 5 minutes to promote complete solubilization of the formazan product. The $400 \mathrm{uL}$ of the purple fomazan-DMSO solution from each well of each plate was then transferred to an individual microcuvette and diluted with $1.1 \mathrm{~mL}$ of deionized water to a final volume of $1.5 \mathrm{~mL}$. The absorbance of each sample at a wavelength of $570 \mathrm{~nm}$ was recorded using an Ultrospec 2000 Pro spectrophotometer. A Student's t-Test was used to test for any statistically significant difference in cell proliferation between serum exposed cells and those not exposed to serum for each cell concentration. The three cell concentrations tested provided six replicates each for serum and serum free conditions. Regression analysis was then used to detect any statistically significant increase in proliferation as cell concentration increased. A concentration of $2.0 \times 10^{5}$ cells $/ \mathrm{mL}$ was determined to be the optimal concentration to observe any possible significant difference between treatment groups. All future cell proliferation and viability experiments were performed in triplicate.

A CCK8 cell proliferation assay similar to that of the MTT assay was performed on the MDA-MB 231, MCF-7, and MCF-12A cells expressing GFP or Rin1 as well as on non- 
transfected cells for each of these cell lines. In this assay, a WST8 (2-(2-methoxy-4nitrophenyl)-3-(4-nitrophenyl)-5-(2,4-disulfophenyl)-2H-tetrazolium, monosodium salt) compound is reduced to an orange, water soluble formazan product that can be detected at a wavelength of $450 \mathrm{~nm}$. As with the MTT assay, the intensity of the absorbance of the colored solution is directly proportional to the number of cells present. All experiments were performed in triplicate for each of the three breast cell lines to determine the effect of serum or IGF-1 stimulation on cellular proliferation. Cells were grown for 24 hours in starvation media on a 12 well plate ( 9 wells in total) at a density of $1.0 \times 10^{5}$ cells per mL with $1 \mathrm{~mL}$ per well. After 24 hours, the starvation media was aspirated from the appropriate wells and replaced with serum containing growth media ( 3 wells) while other wells retained the starvation media ( 3 wells) or were supplemented with $100 \mathrm{ng} / \mathrm{mL}$ of IGF-1 (3 wells). The cells were grown for another 24 hours at $37^{\circ} \mathrm{C}$ with $5 \% \mathrm{CO}_{2}$ and then treated with 25 $\mu \mathrm{L}$ per well of CCK8 solution. Following incubation for 4 hours with CCK8 solution, the growth media was aspirated and its absorbance at $450 \mathrm{~nm}$ was measured. The viability each of the cell samples remaining in the wells was then determined using trypan blue staining.

\section{Trypan Blue Cell Viability Assay}

Following the removal of the growth media and the measurement of its absorbance for cellular proliferation, the cells in each well were washed once with $200 \mathrm{uL}$ of cold 1X PBSEDTA $(1 \mathrm{mM})$ solution. The cells were subsequently detached with $200 \mathrm{uL}$ of trypsinEDTA $(0.25 \%)$ solution for 5 minutes in an incubator at $37^{\circ} \mathrm{C}$ with $5 \% \mathrm{CO}_{2}$. One milliliter of serum free media was added to each well to suspend the cells. A $100 \mathrm{uL}$ sample from each well was subsequently transferred to individual Eppendorf microcentrifuge tubes and mixed with $100 \mathrm{uL}$ of Trypan blue cell viability stain (2X dilution). A $10 \mathrm{uL}$ sample of 
stained cell suspension from each tube was then loaded onto a Bio-Rad dual chamber cell counting slide and the number of cells $/ \mathrm{mL}$ (total and viable) was counted for each sample. A Student's t-Test was used to test for any statistically significant difference in cell viability between serum free, serum exposed, or IGF-1 treated cells.

\section{Western Blotting}

Western blot analysis was performed on the various MDA-MB 231, MCF-7, and MCF12A cell lines prior to conducting any assays to confirm that each cell line expressed the protein(s) of interest. Cells were grown to approximately $80 \%$ confluency in a T75 tissue culture flask containing $15 \mathrm{~mL}$ of $1 \mathrm{X}$ DMEM complete culture media as described in the MTT Cell Proliferation Assay. Cells were then washed once with $1 \mathrm{~mL}$ of cold 1X PBSEDTA $(1 \mathrm{mM})$ and subsequently trypsinized with $1 \mathrm{~mL}$ of Trypsin-EDTA $(0.25 \%)$ solution. One milliliter of $1 \mathrm{X}$ DMEM complete culture media was added to the tissue culture flask to suspend the detached cells. A $100 \mathrm{uL}$ sample of cell suspension was then used to obtain a cell count according to the method outlined above. Following the determination of the cell count per milliliter, $1 \mathrm{~mL}$ of the cell suspension was transferred to a $1.5 \mathrm{~mL}$ Eppendorf microcentrifuge tube and spun (1000X g) for 5 minutes at $4^{\circ} \mathrm{C}$ to obtain a cell pellet. The culture media was then removed and the pellet was washed three times with cold $1 \mathrm{X}$ PBS-EDTA (1mM). One hundred microliters of cold RIPA buffer per $10^{6}$ cells in the pellet was then added to the pellet to lyse the cells. The RIPA buffer was supplemented with Halt Protease and Phosphatase Inhibitor Cocktail (Thermo Scientific, $1 \mathrm{X}$ final concentration). Lysis was achieved by vortexing the pellet in the RIPA buffer for approximately 15 seconds. The cell lysate was then kept on ice for 30 minutes with vortexing every 10 minutes for approximately 15 seconds. After 30 minutes on ice, the cell 
lysate was spun at $10,000 \mathrm{X}$ g for 25 minutes at $4^{0} \mathrm{C}$ in order to pellet the cell debris. The supernatant containing the extracted proteins was then transferred to a clean $1.5 \mathrm{~mL}$ Eppendorf microcentrifuge tube from which it was aliquoted into smaller samples of $50 \mathrm{uL}$ each, and the samples were stored at $-80^{\circ} \mathrm{C}$. The cell lysate protocol described above was obtained from Proteintech and is available at www.ptglab.com.

Prior to Western blotting, the protein concentration of the cell lysate was determined by a bicinchoninic (BCA) protein assay using a kit supplied by Thermo Scientific. A $2 \mathrm{X}$ Laemmli sample buffer was prepared by mixing $950 \mathrm{uL}$ of $2 \mathrm{X}$ Laemmli sample buffer (Bio-Rad) with $50 \mathrm{uL}$ of $\beta$-mercaptoethanol (Bio-Rad). The cell lysate protein sample was prepared for Western blotting by diluting $50 \mathrm{uL}$ of the protein sample with $50 \mathrm{uL}$ of the $2 \mathrm{X}$ Laemmli sample buffer containing $\beta$-mercaptoethanol. The protein sample was then heated for 5 minutes at $95^{\circ} \mathrm{C}$ and $20 \mathrm{uL}$ of denatured protein sample was subsequently loaded onto a 4-20\% gradient polyacrylamide pre-cast gel (Bio-Rad) along with $10 \mathrm{uL}$ of Precision Plus Protein Dual Color Standards (Bio-Rad). Separation of proteins was achieved by running the gel in $1 \mathrm{X}$ running buffer at $200 \mathrm{~V}$ for 30 minutes. $1 \mathrm{X}$ running buffer was prepared by mixing $100 \mathrm{~mL}$ of 10X electrophoresis buffer (25mM Tris, $192 \mathrm{mM}$ glycine, 0.1\% SDS) with $900 \mathrm{~mL}$ of deionized water to a final $\mathrm{pH}$ of 8.3. A Bio-Rad Mini Protean Tetra cell was used to perform the protein electrophoresis. Following electrophoresis, proteins were transferred to a nitrocellulose membrane by running the gel in $1 \mathrm{X}$ transfer buffer for 45 minutes at $100 \mathrm{~V}$. A cold pack and stirring bar were added to the tank containing the transfer buffer and gel cassette to reduce heat which might interfere with protein transfer to the nitrocellulose membrane. Transfer buffer was prepared by mixing $100 \mathrm{~mL}$ of $10 \mathrm{X}$ electrophoresis buffer and $200 \mathrm{~mL}$ of methanol with $700 \mathrm{~mL}$ of deionized water. 
The nitrocellulose membrane containing transferred proteins was then prepared for Western blotting using the Pierce Fast Western Blot Kit (Thermo Scientific) according to the manufacturer's instructions. Briefly, the nitrocellulose membrane blot was washed in 1X Fast Western Wash Buffer to remove any transfer buffer. Ten microliters of primary antibody at a concentration of $1 \mathrm{ug} / \mathrm{uL}$ was then added to $10 \mathrm{~mL}$ of Fast Western Antibody Diluent to produce a final primary antibody dilution of $1: 1000$ or $1 \mathrm{ug} / \mathrm{mL}$. The membrane was then incubated in the primary antibody diluent solution overnight at $4{ }^{\circ} \mathrm{C}$. The membrane was then incubated in $10 \mathrm{~mL}$ of Fast Western Optimized HRP Reagent working dilution for 15 minutes and then subsequently washed four times with 1X Fast Western Wash Buffer for 5 minutes each. The membrane was then incubated in $10 \mathrm{~mL}$ of Detection Reagent working solution for 5 minutes after which it was prepared for imaging by exposure to $\mathrm{X}$ ray film. The primary antibodies used were a rabbit polyclonal anti-Rin1 (human) antibody (Proteintech, 16388-1-AP) and a rabbit eGFP tag antibody (Pierce Thermo Scientific, CAB4211). A GAPDH antibody (Pierce Thermo Scientific, MA516034) was used as a loading control.

\section{Immunofluorescence}

MDA-MB 231 breast cancer cells expressing GFP or Rin1 were seeded onto coverslips submerged in $1 \mathrm{~mL}$ of selection media within the wells of a 12 well plate. The cells were incubated overnight at $37^{\circ} \mathrm{C}$ and $5 \% \mathrm{CO}_{2}$ in order to attach to the coverslips. The following day the growth media was aspirated from each well and replaced with a $4 \%$ formaldehyde solution. The samples were allowed to fix in the formaldehyde solution for 15 minutes at room temperature. The fixative was then removed and the samples were washed three times in $1 \mathrm{X}$ PBS for 5 minutes each. The cell samples were then blocked for one hour in blocking 
buffer containing $5 \%$ normal goat serum and $0.3 \%$ Triton-X 100. Following the blocking step, the samples were incubated overnight at $4^{\circ} \mathrm{C}$ in primary antibody diluted in antibody buffer containing $1 \%$ BSA and $0.3 \%$ Triton-X 100 . The Rin 1 and GFP primary antibodies used were the same as those utilized for Western blotting and were diluted at a ratio of 1 $\mathrm{uL} \mathrm{IgG} / 200 \mathrm{uL}$ buffer. The following day the samples were washed three times in $1 \mathrm{X}$ PBS for 5 minutes each and then incubated in an Alexafluor488 conjugated secondary antibody (goat anti-rabbit) for one hour at room temperature in the dark. The secondary antibody was diluted at a ratio of $1 \mathrm{uL} \mathrm{IgG/300} \mathrm{uL}$ buffer. The coverslips containing the cell samples were then mounted to glass microscope slides using Prolong Gold Antifade reagent with DAPI. The samples were allowed to cure overnight at room temperature in the dark. The samples were then stored at $4^{\circ} \mathrm{C}$ until imaged with an Olympus confocal microscope.

\section{Telomerase Activity Assay}

The MDA-MB 231 human breast cancer cells expressing GFP and Rin1 as well as nontransfected cells were serum starved for 24 hours at $37^{\circ} \mathrm{C}$ and $5 \% \mathrm{CO}_{2}$ on a 12 well plate at a density of $1.0 \times 10^{5}$ cells per well in $1 \mathrm{~mL}$ of serum free growth media. Each MDAMB 231 cell line was plated in duplicate. After 24 hours, $100 \mathrm{ng} / \mathrm{mL}$ of IGF-1 was added to half of the total samples- one well per cell type. The other half of the samples did not receive IGF-1 treatment. The samples were then incubated for another 24 hours, after which time the culture media in each well was removed and the cells were subsequently prepared for the telomeric repeat amplification protocol (TRAP) assay as per the manufacturer's instructions. The TRAPeze Telomerase Detection Kit (EMD Millipore, S7700) was utilized to determine the telomerase activity in each of the IGF-1 treated and untreated samples for the three MDA-MB 231 cell types mentioned above. 
Briefly, cells were lysed for 30 minutes in cold 1X CHAPS lysis buffer supplemented with $10 \mathrm{uL} / \mathrm{mL}$ of protease and phosphatase inhibitor to extract the telomerase enzyme. The cell lysate was then centrifuged at $10,000 \mathrm{rpm}$ for 15 minutes at $4^{\circ} \mathrm{C}$. Protein extract from each cell sample was then added to a $\mathrm{qPCR}$ master mix containing a telomerase substrate (TS) molecule which was subsequently extended by the telomerase enzyme to create a ladder of DNA products of various lengths starting at $50 \mathrm{bp}$. The qPCR master mix consisted of the following per reaction: 10X TRAP reaction buffer ( $5 \mathrm{uL}), 50 \mathrm{X}$ DNTP mix ( $1 \mathrm{uL})$, TS primer $(1 \mathrm{uL})$, TRAP primer mix ( $1 \mathrm{uL})$, Taq polymerase (2 units, $0.4 \mathrm{uL})$, EvaGreen dye $(2.5 \mu \mathrm{L})$ and $\mathrm{dH}_{2} \mathrm{O}(40 \mathrm{uL})$. The telomerase extended DNA products were then amplified and quantified by qPCR. The qPCR amplification protocol consisted of the following steps: 1) $30^{\circ} \mathrm{C}$ for 30 minutes, 2) $95^{\circ} \mathrm{C}$ for 2 minutes, 3) $94^{\circ} \mathrm{C}$ for 15 seconds, 4) $59^{\circ} \mathrm{C}$ for 30 seconds, 5 ) $72^{\circ} \mathrm{C}$ for 1 minute and 6) plate read. Steps three through six was repeated for a total of 40 cycles. The DNA products were visualized by ethidium bromide staining $(0.5 \mathrm{ug} / \mathrm{mL})$ using a FotoDyne imager with an ethidium bromide filter. The gel was stained for 30 minutes in $200 \mathrm{~mL}$ of the ethidium bromide solution and then de-stained for 30 minutes in deionized $\mathrm{H}_{2} \mathrm{O}$ prior to imaging. The water was changed every 10 minutes during the de-staining process. The telomerase activity of MCF-7 and MCF-12A cell lines expressing either GFP or Rin1 as well as non-transfected cells was determined in the same manner as described above for the MDA-MB 231 cell lines.

\section{Telomerase Gene Expression}

Telomerase gene expression levels in the MDA-MB 231, MCF-7, and MCF-12A breast cell lines expressing GFP and Rin1 were measured by reverse transcriptase qPCR (RTqPCR). Cells were plated on a 24 well plate at a density of $2.0 \times 10^{5}$ cells per $\mathrm{mL}$ in one 
$\mathrm{mL}$ of serum free DMEM culture media. A total of two wells were plated for each breast cell line. After 24 hours of incubation at $37{ }^{\circ} \mathrm{C}$ with $5 \% \mathrm{CO}_{2}, 100 \mathrm{ng} / \mathrm{mL}$ of IGF-1 was added to one of the wells while the other well received no IGF-1 treatment. The cells were then incubated for another 24 hours and then lysed according to the manufacturer's instructions to extract total RNA using the Invitrogen PureLink RNA Mini Kit (catalog\# 12183018A). The RNA concentration of each cell sample was then determined by established spectrophotometric methods using the A260/A280 and A260/A230 ratios. cDNA synthesis to measure gene expression levels of telomerase and GAPDH (control) was then performed in duplicate according to the manufacturer's instructions using the SuperScript III RT-PCR system from Invitrogen (catalog\# 18080093). Briefly, a qPCR master mix was prepared consisting of the following per reaction: $2 \mathrm{X}$ reaction mix $(25 \mu \mathrm{L})$, forward and reverse primers ( $1 \mu \mathrm{L}$ each), SuperScript III RT/Platinum Taq Mix $(2 \mu \mathrm{L})$, RNA template $(0.1 \mu \mathrm{g})$, EvaGreen dye $(2.5 \mu \mathrm{L})$, and PCR grade water to a final volume of $50 \mu \mathrm{L}$. The RT-qPCR protocol consisted of the following steps: 1) $55^{\circ} \mathrm{C}$ for 30 minutes, 2) $94^{\circ} \mathrm{C}$ for 2 minutes, 3) $94^{\circ} \mathrm{C}$ for 15 seconds, 4) $53^{\circ} \mathrm{C}$ for 30 seconds, 5) $68^{\circ} \mathrm{C}$ for 1 minute, and 6) plate read. Steps three through six were repeated for a total of 40 cycles. A final extension step of $68^{\circ} \mathrm{C}$ for 5 minutes was carried out before a melt curve analysis. The GAPDH and telomerase qPCR products were then separated on a $12 \%$ acrylamide gel run at $125 \mathrm{~V}$ for one hour in $1 \mathrm{X}$ TBE buffer and subsequently visualized by ethidium bromide staining using a Fotodyne digital imager. 


\subsection{References}

Ayub, A., Yip, W., \& Seow, H.F. (2015). Dual treatments targeting IGF-1R, PI3K, mTORC or MEK synergize to inhibit cell growth, induce apoptosis, and arrest cell cycle at G1 phase in MDA-MB-231 cell line. Biomedicine \& Pharmacotherapy, 75, 40-50.

Badache, A., \& Hynes, N. E. (2001). Interleukin 6 inhibits proliferation and, in cooperation with an epidermal growth factor receptor autocrine loop, increases migration of T47D breast cancer cells. Cancer Research, 61(1), 383-391.

Fox, E. M., Andrade, J., \& Shupnik, M. A. (2009). Novel actions of estrogen to promote proliferation: Integration of cytoplasmic and nuclear pathways. Steroids, 74(7), 622627.

Helbig, G., Christopherson, K., Bhat-Nakshatri, P., Kumar, S., Kishimoto, H., Miller, K., \& Nakshatri, H. (2003). NF- $\kappa$ B promotes breast cancer cell migration and metastasis by inducing the expression of the chemokine receptor CXCR4. Journal of Biological Chemistry, 278(24), 21631.

Kim, R. K., Suh, Y., Yoo, K. C., Cui, Y. H., Kim, H., Kim, M. J., \& Lee, S. J. (2015). Activation of KRAS promotes the mesenchymal features of basal-type breast cancer. Experimental \& molecular medicine, 47(1), e137.

Lazennec, G., Bresson, D., Lucas, A., Chauveau, C., \& Vignon, F. (2001). ER $\beta$ inhibits proliferation and invasion of breast cancer cells. Endocrinology, 142(9), 4120-4130.

Liao, G., Wang, M., Ou, Y., \& Zhao, Y. (2014). IGF-1-induced epithelial-mesenchymal transition in MCF-7 cells is mediated by MUC1. Cellular Signaling, 26(10), 21312137.

Lu, Y., Zi, X., Zhao, Y., \& Pollak, M. (2004). Overexpression of ErbB2 receptor inhibits IGF-I-induced Shc-MAPK signaling pathway in breast cancer cells. Biochemical and Biophysical Research Communications, 313(3), 709-715.

Malaguarnera, R., \& Belfiore, A. (2011). Insulin receptor and cancer. Endocrine-Related Cancer, 18(4), 125-147.

Mawson, A., Lai, A., Carroll, J. S., Sergio, C. M., Mitchell, C. J., \& Sarcevic, B. (2005). Estrogen and insulin/IGF-1 cooperatively stimulate cell cycle progression in MCF-7 breast cancer cells through differential regulation of c-myc and cyclin D1. Molecular and Cellular Endocrinology, 229(1), 161-173.

Mezi, S., Todi, L., Orsi, E., Angeloni, A., \& Mancini, P. (2012). Involvement of the srccortactin pathway in migration induced by IGF-1 and EGF in human breast cancer cells. International Journal of Oncology, 41(6), 2128-2138. 
Morimura, S., \& Takahashi, K. (2011). Rac1 and stathmin but not EB1 are required for invasion of breast cancer cells in response to IGF-I. International Journal of Cell Biology, 2011, 1-9.

Mukohara, T., Shimada, H., Ogasawara, N., Wanikawa, R., Shimomura, M., Nakatsura, T., Ishii, G., Park, J., Jänne, P., Saijo, N., \& |Minami, H. (2009). Sensitivity of breast cancer cell lines to the novel insulin-like growth factor-1 receptor (IGF-1R) inhibitor NVP-AEW541 is dependent on the level of IRS-1 expression. Cancer Letters, 282(1), 14-24.

Park, J., Choi, S., Lee, J., Lee, M., Nam, E., Jeong, A., \& Yang, Y. (2013). Interleukin$32 \beta$ stimulates migration of MDA-MB-231 and MCF-7cells via the VEGF-STAT3 signaling pathway. Cellular Oncology, 36(6), 493-503.

Pandey, D. P., Picard, D., Madeo, A., Maggiolini, M., Lappano, R., \& Albanito, L. (2009). Estrogenic GPR30 signalling induces proliferation and migration of breast cancer cells through CTGF. The EMBO Journal, 28(5), 523-532.

Price, J. T., Tiganis, T., Agarwal, A., Djakiew, D., \& Thompson, E. W. (1999). Epidermal growth factor promotes MDA-MB-231 breast cancer cell migration through a phosphatidylinositol 3'-kinase and phospholipase C-dependent mechanism. Cancer Research, 59(21), 5475-5478.

Riedemann, J., Sohail, M., \& Macaulay, V. M. (2007). Dual silencing of the EGF and type 1 IGF receptors suggests dominance of IGF signaling in human breast cancer cells. Biochemical and Biophysical Research Communications, 355(3), 700-706.

Sánchez-Bailón, M. P., Calcabrini, A., Gómez-Domínguez, D., Morte, B., Martín-Forero, E., Gómez-López, G., \& Martín-Pérez, J. (2012). Src kinases catalytic activity regulates proliferation, migration and invasiveness of MDA-MB-231 breast cancer cells. Cellular Signaling, 24(6), 1276-1286.

Sarkar, S., Maceyka, M., Hait, N. C., Paugh, S. W., Sankala, H., Milstien, S., \& Spiegel, S. (2005). Sphingosine kinase 1 is required for migration, proliferation and survival of MCF-7 human breast cancer cells. FEBS Letters, 579(24), 5313-5317.

Son, J., Lee, J., Lee, Z. H., Kim, H., \& Ha, H. (2010). cAMP-response-element-binding protein positively regulates breast cancer metastasis and subsequent bone destruction. Biochemical and Biophysical Research Communications, 398(2), 309314.

Santen, R. J., Song, R. X., Chen, Y., Zhang, Z., Bao, Y., Yue, W., \& Wang, J. (2010). Estrogen utilization of IGF-1-R and EGF-R to signal in breast cancer cells. Journal of Steroid Biochemistry and Molecular Biology, 118(4), 219-230.

Song, X., Wei, Z., \& Shaikh, Z. A. (2015). Requirement of ER $\alpha$ and basal activities of EGFR and src kinase in cd-induced activation of MAPK/ERK pathway in human breast cancer MCF-7 cells. Toxicology and Applied Pharmacology, 287(1), 26-34. 
Taliaferro-Smith, L., Oberlick, E., Liu, T., McGlothen, T., Alcaide, T., Tobin, R., \& O'Regan, R. (2015). FAK activation is required for IGF1R-mediated regulation of EMT, migration, and invasion in mesenchymal triple negative breast cancer cells. Oncotarget, 6(7), 4757.

Tecalco-Cruz, A.C. \& Ramírez-Jarquín, J. O. (2016). Mechanisms that increase stability of estrogen receptor alpha in breast cancer. Clinical Breast Cancer, 17(1), 1-10.

Ulanet, D. B., Ludwig, D.L., Kahn, C. R., Hanahan, D., \& Hunter, Tony. (2010). Insulin receptor functionally enhances multistage tumor progression and conveys intrinsic resistance to IGF-1R targeted therapy. Proceedings of the National Academy of Sciences of the United States of America, 107(24), 10791-10798.

Verbeek, B. S., Adriaansen-Slot, S. S., Vroom, T. M., Beckers, T., \& Rijksen, G. (1998). Overexpression of EGFR and c-erbB2 causes enhanced cell migration in human breast cancer cells and NIH3T3 fibroblasts. FEBS Letters, 425(1), 145-150.

Voudouri, K., Nikitovic, D., Berdiaki, A., Kletsas, D., Karamanos, N. K., \& Tzanakakis, G. N. (2016). IGF-I/EGF and E2 signaling crosstalk through IGF-IR conduit point affects breast cancer cell adhesion. Matrix Biology, 56, 95-113.

Walsh, L. A., \& Damjanovski, S. (2011). IGF-1 increases invasive potential of MCF 7 breast cancer cells and induces activation of latent TGF- $\beta 1$ resulting in epithelial to mesenchymal transition. Cell Communication and Signaling: CCS, 9(1), 10.

Zhang, X., Deng, H., \& Wang, Z. (2014). Estrogen activation of the mitogen-activated protein kinase is mediated by ER- $\alpha 36$ in ER-positive breast cancer cells. Journal of Steroid Biochemistry and Molecular Biology, 143, 434-443.

Zhu, C., Qi, X., Chen, Y., Sun, B., Dai, Y., \& Gu, Y. (2011). PI3K/akt and MAPK/ERK1/2 signaling pathways are involved in IGF-1-induced VEGF-C upregulation in breast cancer. Journal of Cancer Research and Clinical Oncology, 137(11), 1587-1594. 


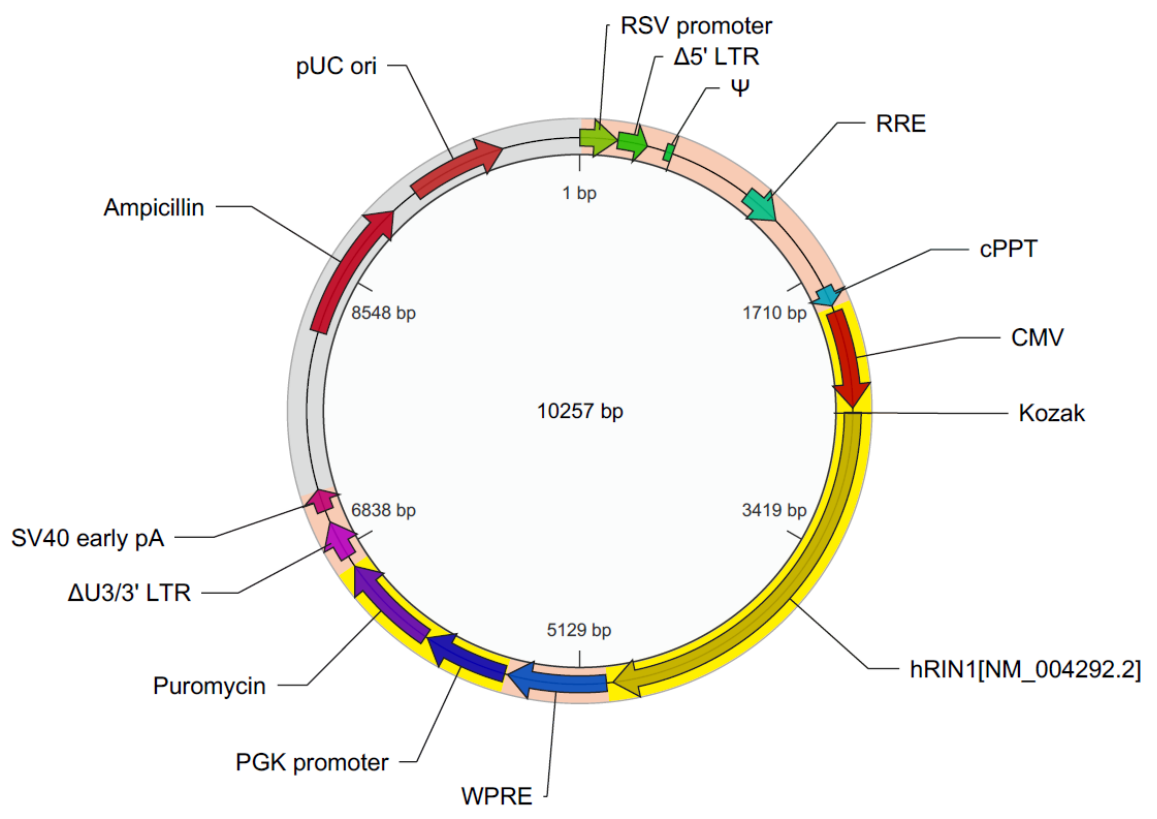

Figure 2.1: Lentiviral vector used to create the MDA-MB 231 cell line expressing Rin1. The Rin1 gene sequence is inserted directly downstream of the CMV promoter. An identical vector with the GFP gene sequence inserted was used to create the MDA-MB 231 cell line expressing GFP. Also labeled are the various elements necessary for lentivirus production and cell selection. 

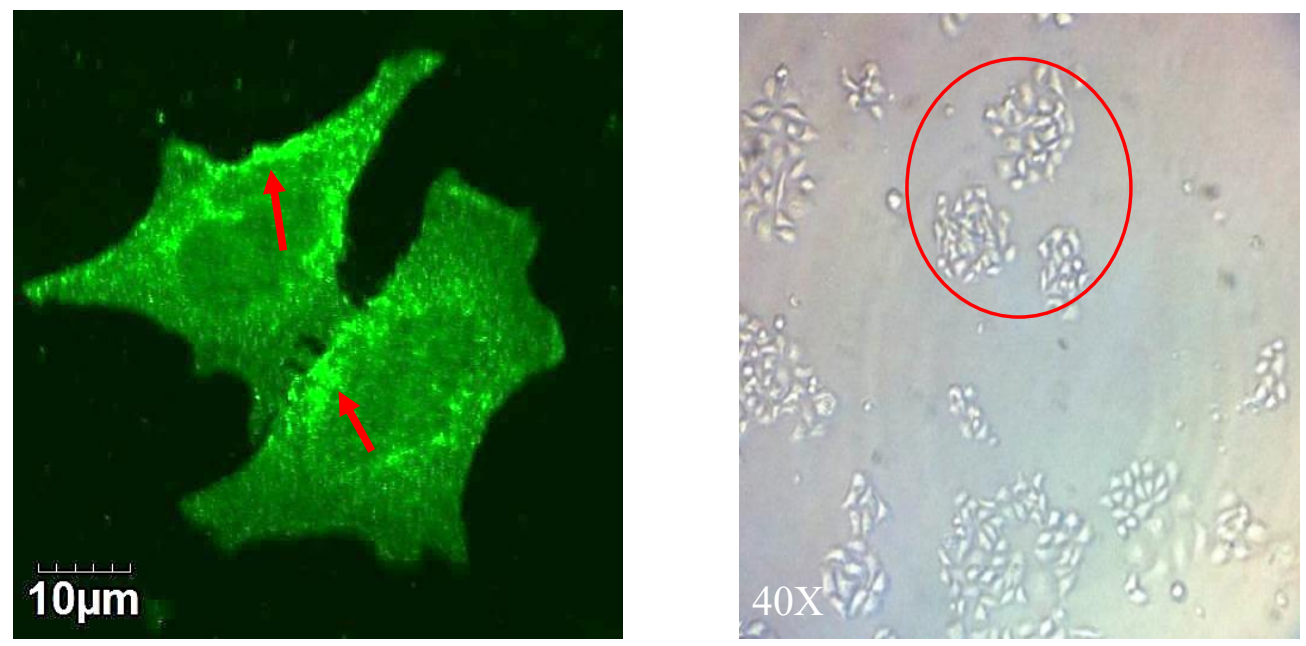

A.

B.

Figure 2.2: A) Confocal micrograph image of MDA-MB 231 cells expressing Rin1. Rin1 tends to localize near the plasma membrane (red arrows). B) Light micrograph image illustrating the clumped growth pattern of MDA-MB 231 cells expressing Rin1 (red circle).

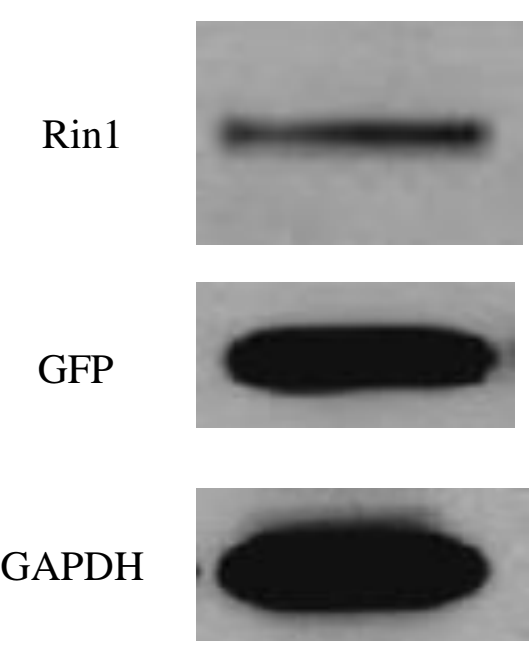

A.
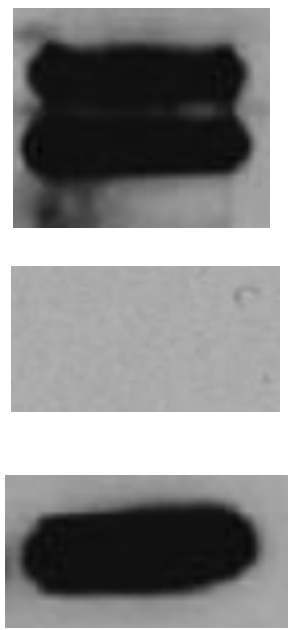

B.

Figure 2.3: Western blot image of the A) MDA-MB 231 GFP cell line expressing GFP and endogenous levels of Rin1. B) MDA-MB 231 cell line overexpressing Rin1. 


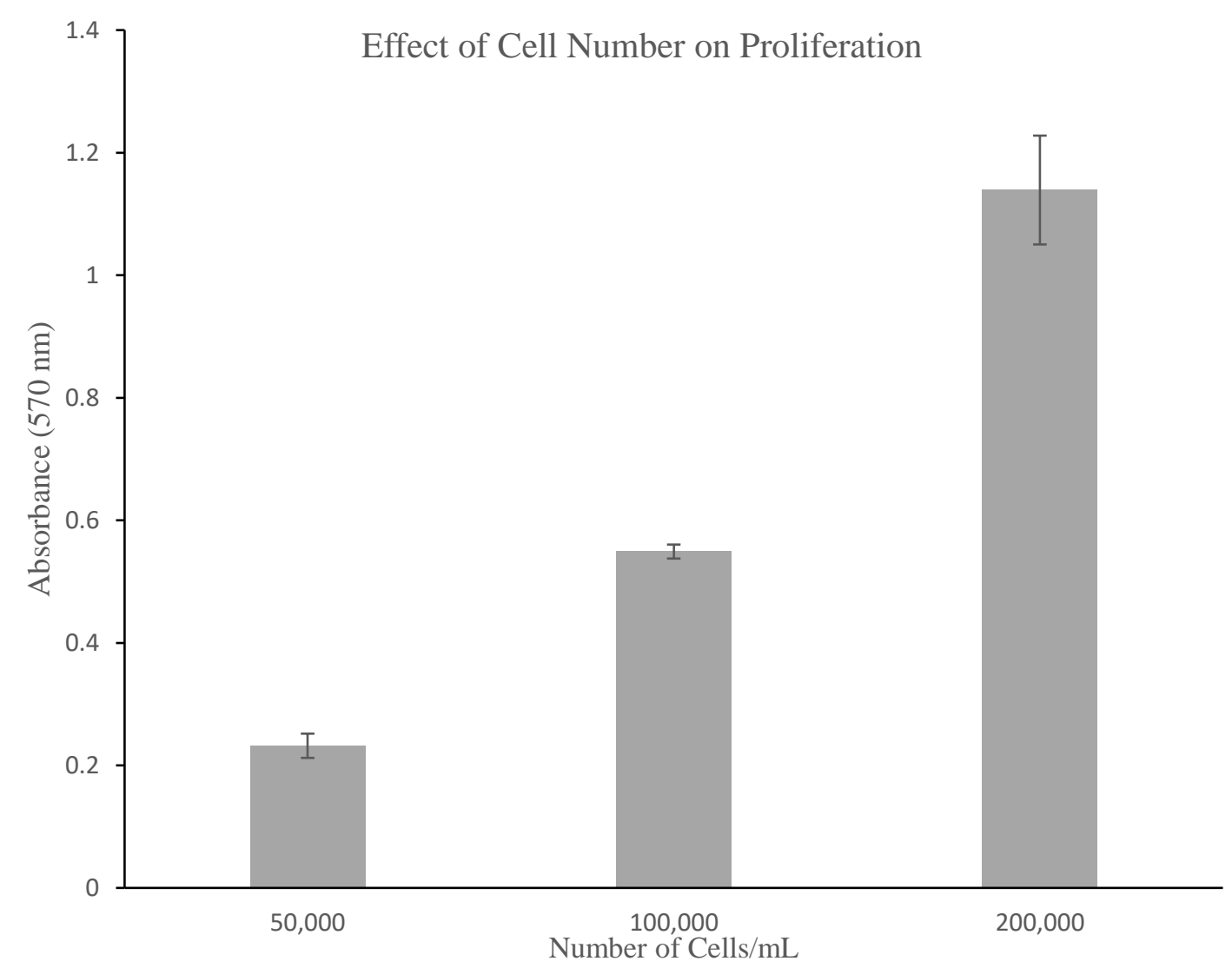

Figure 2.4: The effect of cell number on cellular proliferation in serum exposed MDAMB 231 cells. Error bars represent the standard error of the mean. 


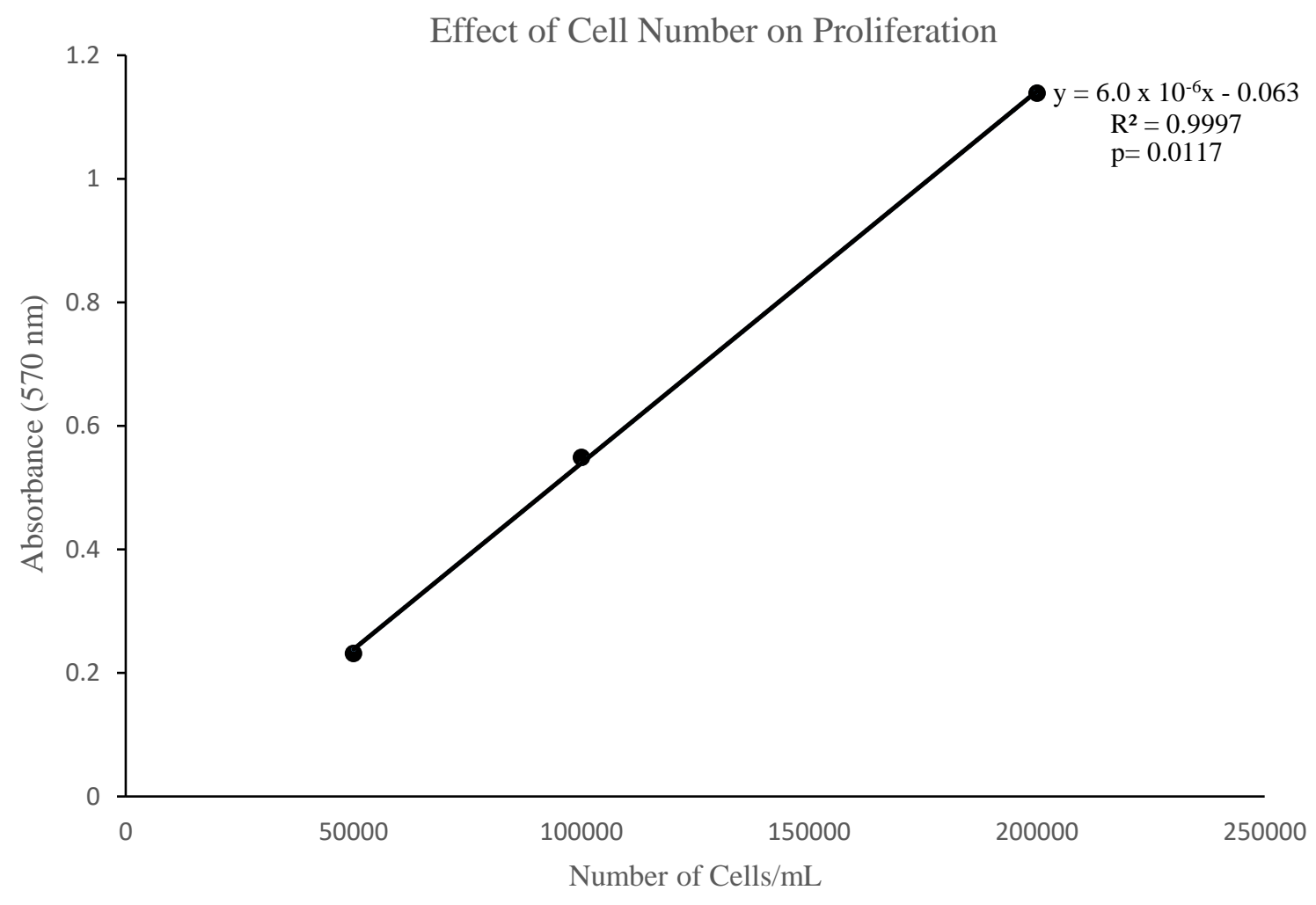

Figure 2.5: Regression analysis of the effect of cell number on cellular proliferation in serum exposed MDA-MB 231 cells. 
Effect of IGF-1 Concentration on Cellular Proliferation

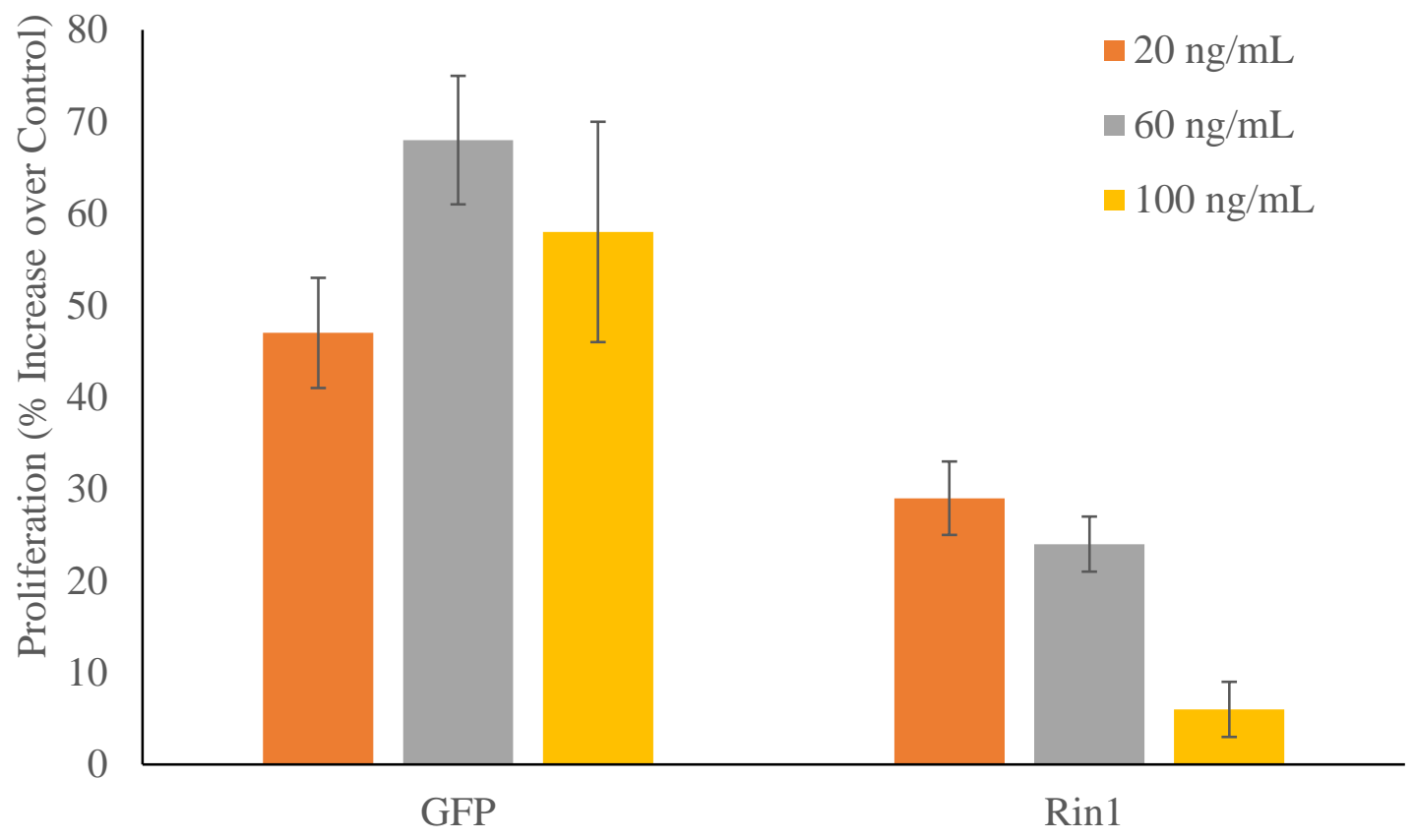

Figure 2.6: The effect of IGF-1 concentration on cellular proliferation in MDA-MB 231 cells expressing GFP or Rin1. Values represent the percent increase in cellular proliferation over an unstimulated $(0 \mathrm{ng} / \mathrm{mL})$ control. All experiments were conducted in triplicate. Error bars represent standard error of the mean. 


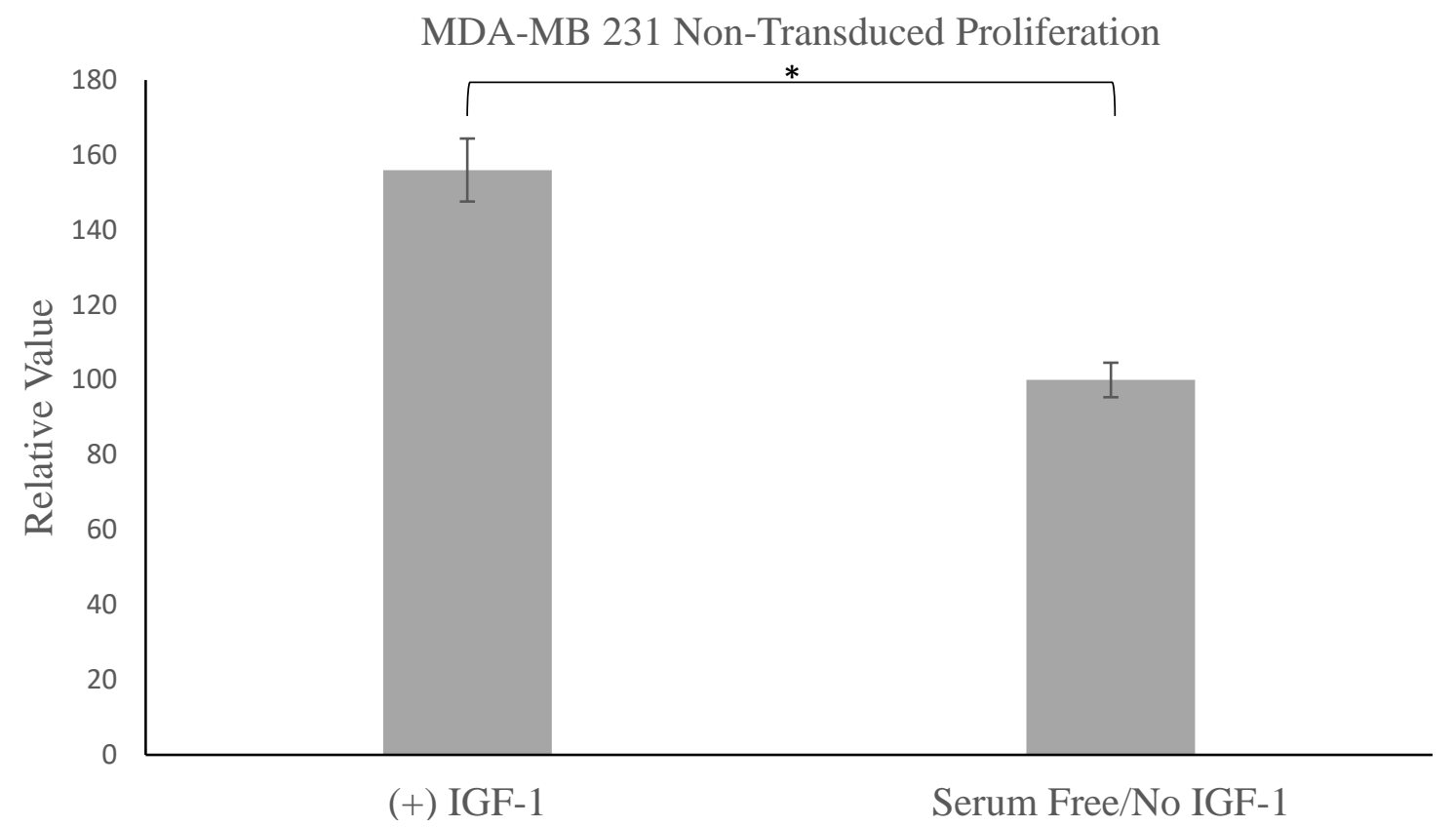

Figure 2.7: The effect of IGF-1 stimulation $(100 \mathrm{ng} / \mathrm{mL})$ on cellular proliferation of non-transduced MDA-MB 231 cells. Values represent the percent increase normalized to the serum free/no IGF-1 treatment group. All experiments were conducted in triplicate. Error bars represent the standard error of the mean.

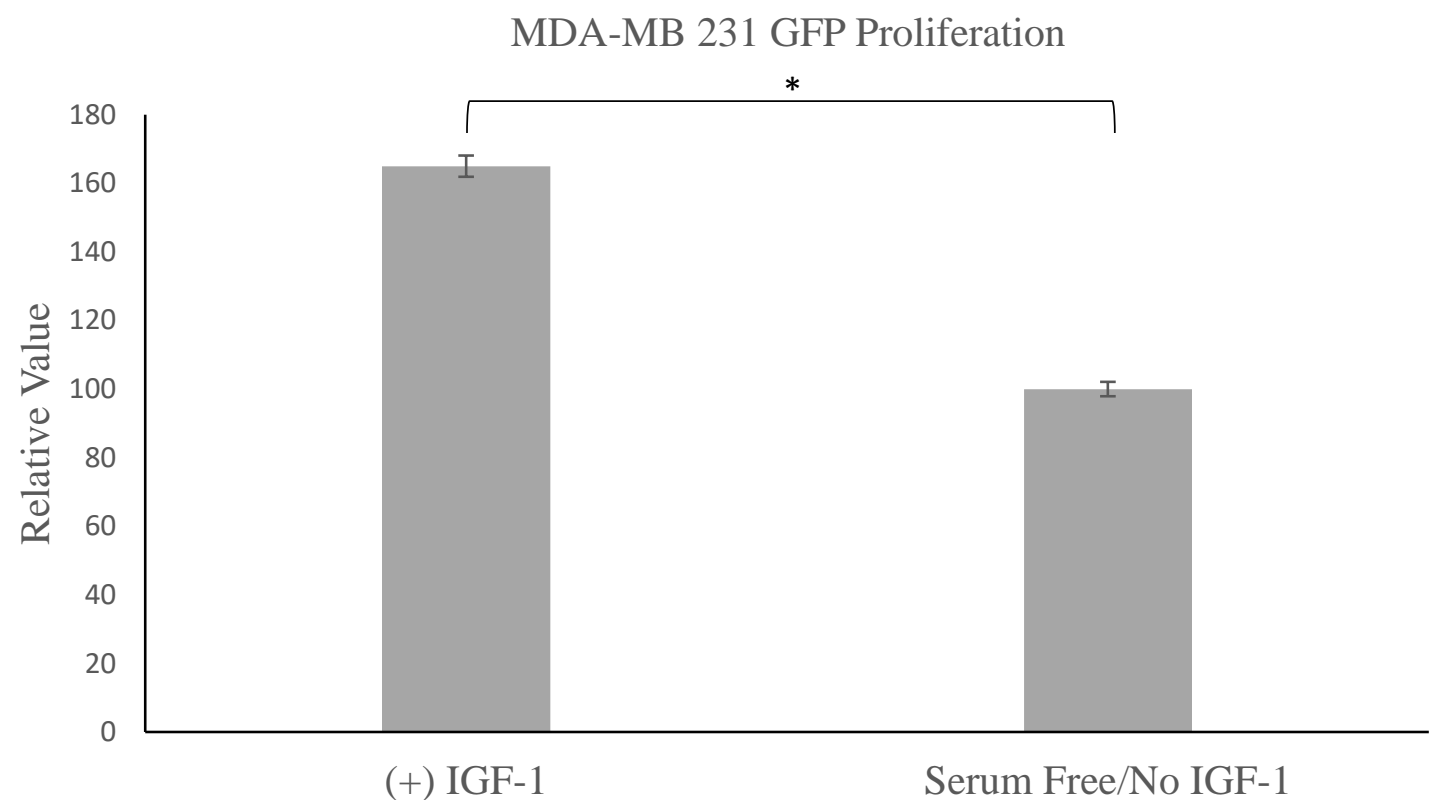

Figure 2.8: The effect of IGF-1 stimulation $(100 \mathrm{ng} / \mathrm{mL})$ on cellular proliferation of MDA-MB 231 cells expressing GFP. Values represent the percent increase normalized to the serum free/no IGF-1 treatment group. All experiments were conducted in triplicate. Error bars represent the standard error of the mean. 


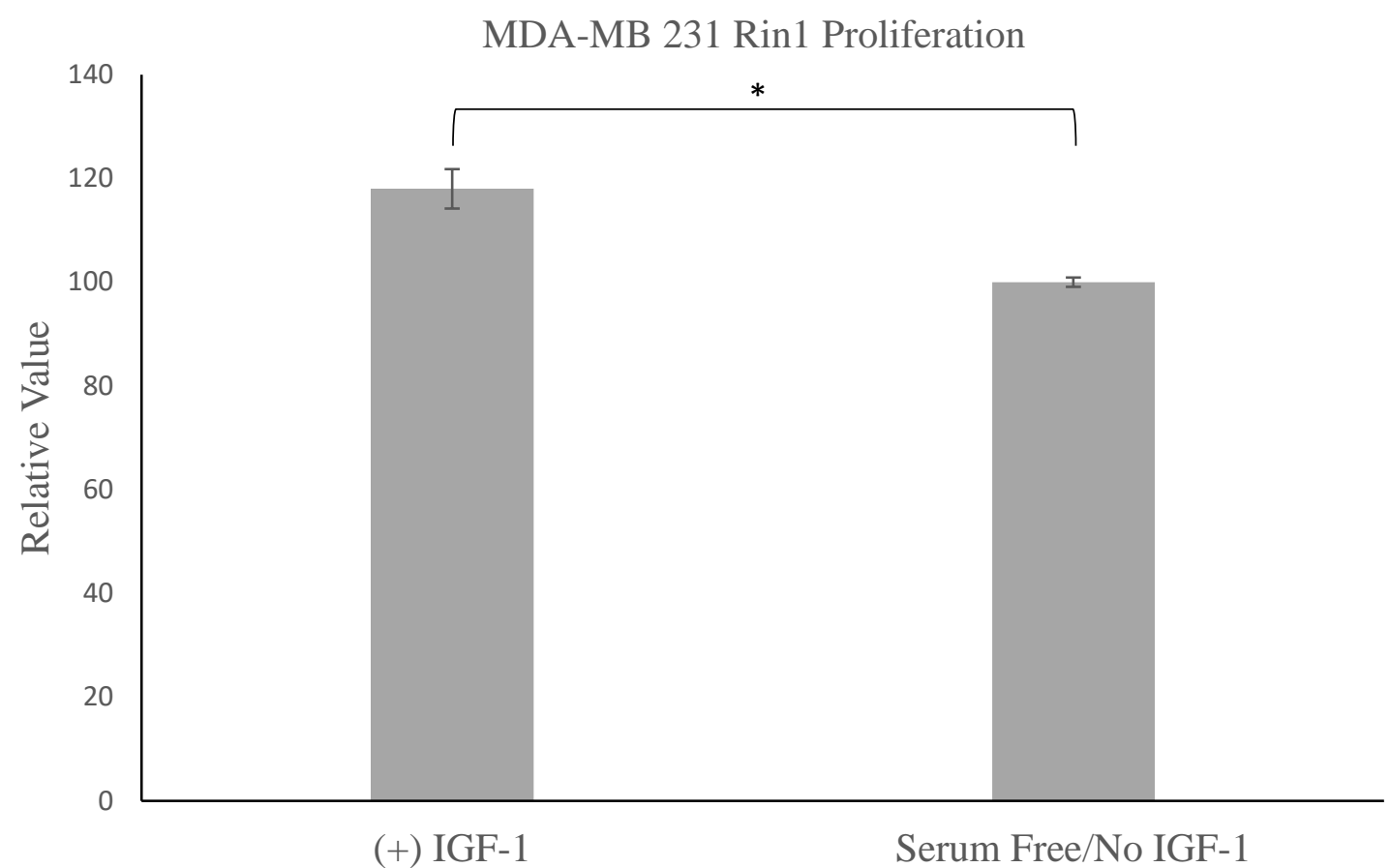

Figure 2.9: The effect of IGF-1 stimulation $(100 \mathrm{ng} / \mathrm{mL})$ on cellular proliferation of MDA-MB 231 cells expressing Rin1. Values represent the percent increase normalized to the serum free/no IGF-1 treatment group. All experiments were conducted in triplicate. Error bars represent the standard error of the mean.

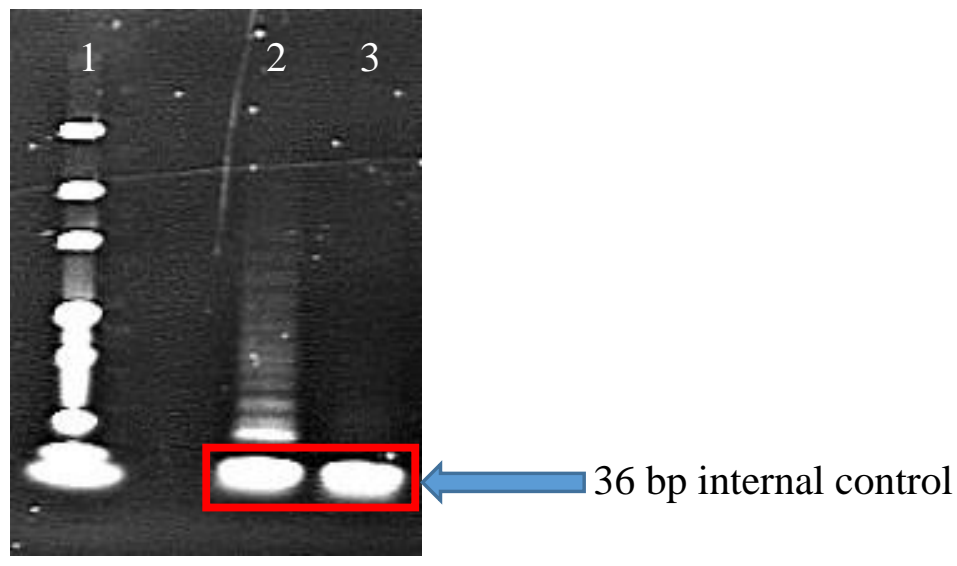

Figure 2.10: Telomerase activity assay demonstrating the presence of telomeric repeat amplification protocol PCR products. 1. 25 bp DNA ladder. 2. HeLa cell telomerase positive control. 3. HeLa cell heat treated telomerase negative control. Red box is the 36 base pair internal control. 


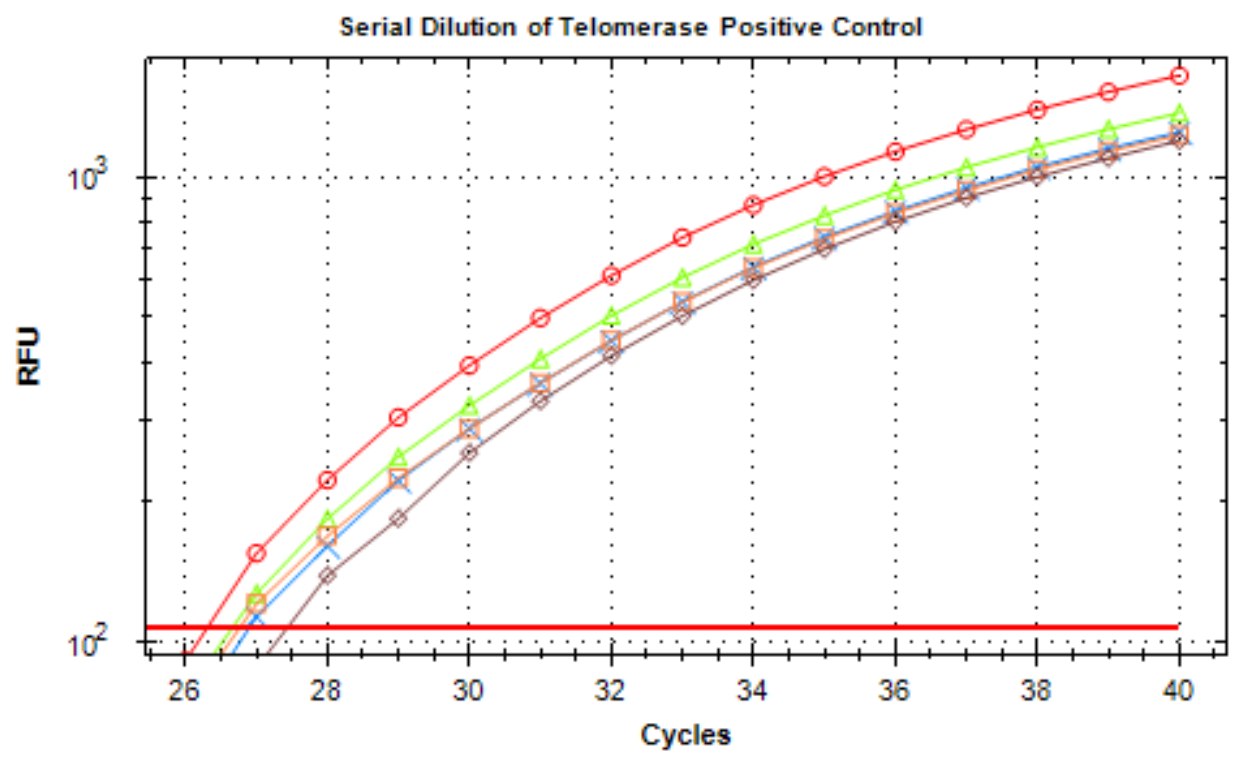

Figure 2.11: Amplification curves of the qPCR telomerase activity products for the serial dilution of a HeLa cell telomerase positive control sample. Red circle is undiluted sample, green triangle is $10 \mathrm{X}$ dilution, orange square is $100 \mathrm{X}$ dilution, blue cross is $1,000 \mathrm{X}$ dilution, and brown diamond is buffer only.

\begin{tabular}{|c|c|}
\hline Serial Dilution of HeLa Cell Telomerase Positive Control \\
\hline Average Ct Value \pm Standard Error \\
\hline Undiluted & $26.28 \pm 0.02$ \\
\hline $10 X$ & $26.50 \pm 0.11$ \\
\hline $100 X$ & $26.70 \pm 0.03$ \\
\hline $1,000 X$ & $26.78 \pm 0.10$ \\
\hline Buffer & $27.02 \pm 0.37$ \\
\hline
\end{tabular}

Table 2.1: Average cycle threshold $(\mathrm{Ct})$ values of the qPCR amplification of telomerase activity products for the serial dilution of a HeLa cell telomerase positive control sample. All experiments were conducted in duplicate. 


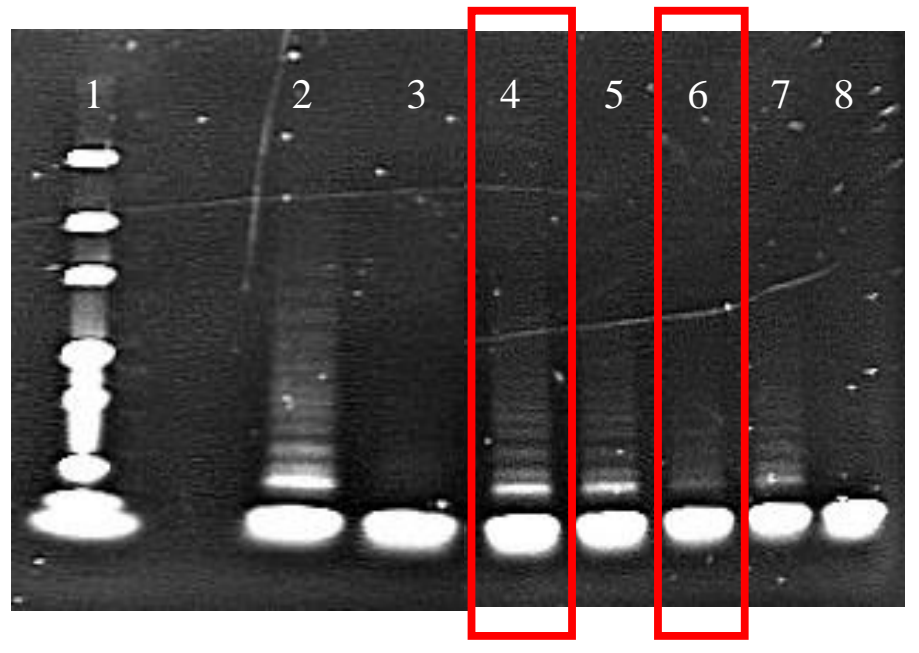

Figure 2.12: PAGE gel analysis of the telomerase activity qPCR TRAP products for the MDA-MB 231 cell lines following stimulation with $100 \mathrm{ng} / \mathrm{mL}$ of IGF-1 (+) for 24 hours or no stimulation (-). 1. 25 bp ladder. 2. Telomerase positive control. 3 . Telomerase heat treated negative control. 4. GFP (+) IGF-1. 5. GFP (-) IGF-1. 6. Rin1 (+) IGF-1. 7. Rin1 (-) IGF-1. 8. Buffer. Red boxes indicate reduced telomerase activity in cells expressing Rin1 when compared to cells expressing GFP following IGF-1 stimulation. 


\begin{tabular}{|c|c|}
\hline \multicolumn{2}{|c|}{ MDA-MB 231 Telomerase Activity } \\
\hline & Average $\mathrm{Ct}$ Value \pm Standard Error \\
\hline Telomerase positive control & $19.92 \pm 0.13$ \\
\hline Telomerase negative control & $20.16 \pm 0.15$ \\
\hline GFP (+) IGF-1 & $20.49 \pm 0.01$ \\
\hline GFP (-) IGF-1 & $20.41 \pm 0.04$ \\
\hline Rin1 (+) IGF-1 & $21.14 \pm 0.02$ \\
\hline Rin1 (-) IGF-1 & $20.68 \pm 0.01$ \\
\hline Buffer & $20.23 \pm 0.09$ \\
\hline $\begin{array}{l}21.14-20.49=0.6 \\
2^{-0.65}=0.64 \\
1 / 0.64=1.56 \\
1.56 \text { fold decreas } \\
\text { expressing Rin } 1\end{array}$ & $\begin{array}{l}\text { lomerase activity for cells } \\
\text { pared to cells expressing GFP. }\end{array}$ \\
\hline
\end{tabular}

Table 2.2: Cycle threshold values $(\mathrm{Ct})$ for the $\mathrm{qPCR}$ products of telomerase activity in MDA-MB 231 cell lines following stimulation with $100 \mathrm{ng} / \mathrm{mL}$ of IGF-1 (+) for 24 hours or no stimulation (-). All experiments were conducted in duplicate. 
1. GFP (+) IGF-1

2. GFP (-) IGF-1

3. Rin1 WT (+) IGF-1

4. Rin1 WT (-) IGF-1

5. Buffer (no RNA)

\section{MDA-MB 231 Gene Expression}

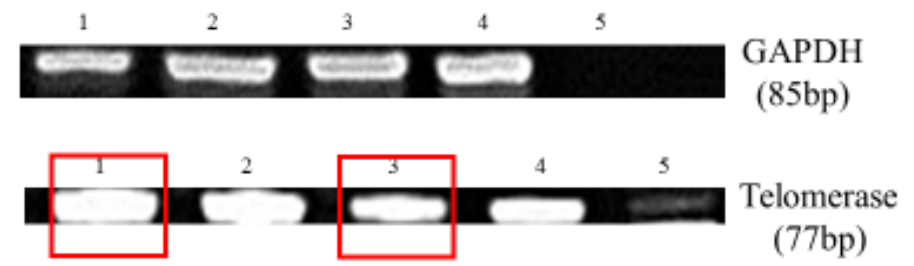

Figure 2.13: PAGE gel analysis of the RT-qPCR GAPDH and telomerase gene expression products in the MDA-MB 231 cell lines following stimulation with 100 $\mathrm{ng} / \mathrm{mL}$ of IGF-1 (+) for 24 hours or no stimulation (-).

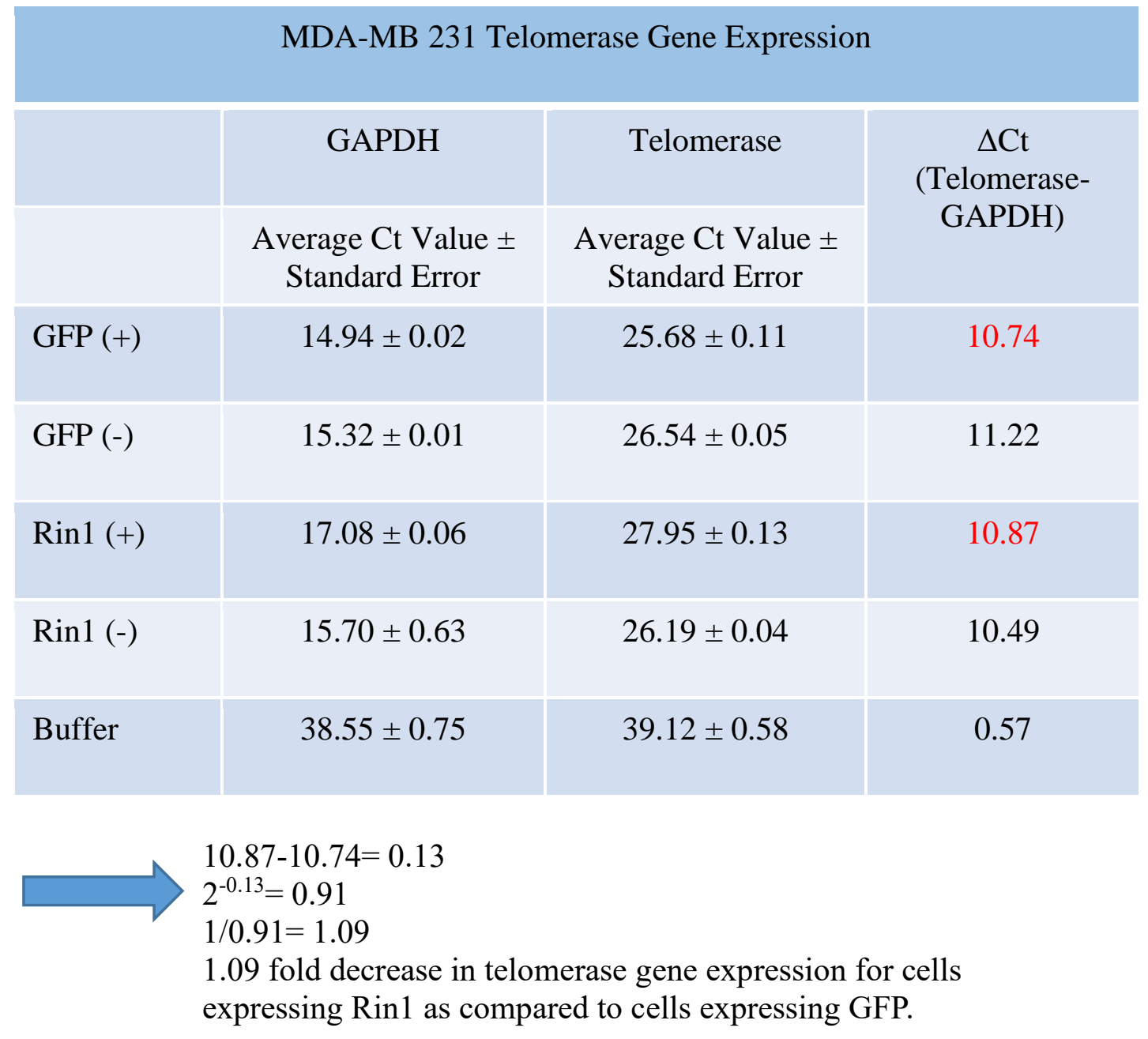

Table 2.3: Cycle threshold values $(\mathrm{Ct})$ for the RT-qPCR of telomerase gene expression in MDA-MB 231 cell lines following stimulation with $100 \mathrm{ng} / \mathrm{mL}$ of IGF-1 (+) for 24 hours or no stimulation (-). All experiments were conducted in duplicate. 


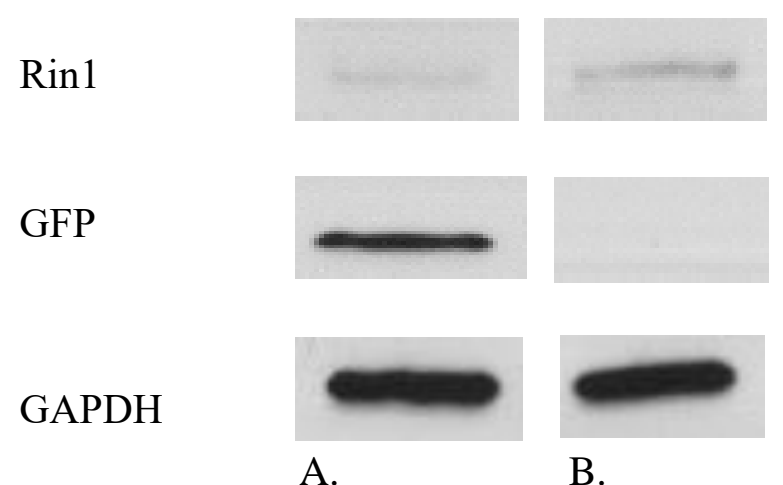

Figure 2.14: Western blot image of the A) MCF-12A GFP cell line expressing GFP and endogenous levels of Rin1. B) MCF-12A cell line overexpressing Rin1.

MCF-12A Non-Transduced Proliferation

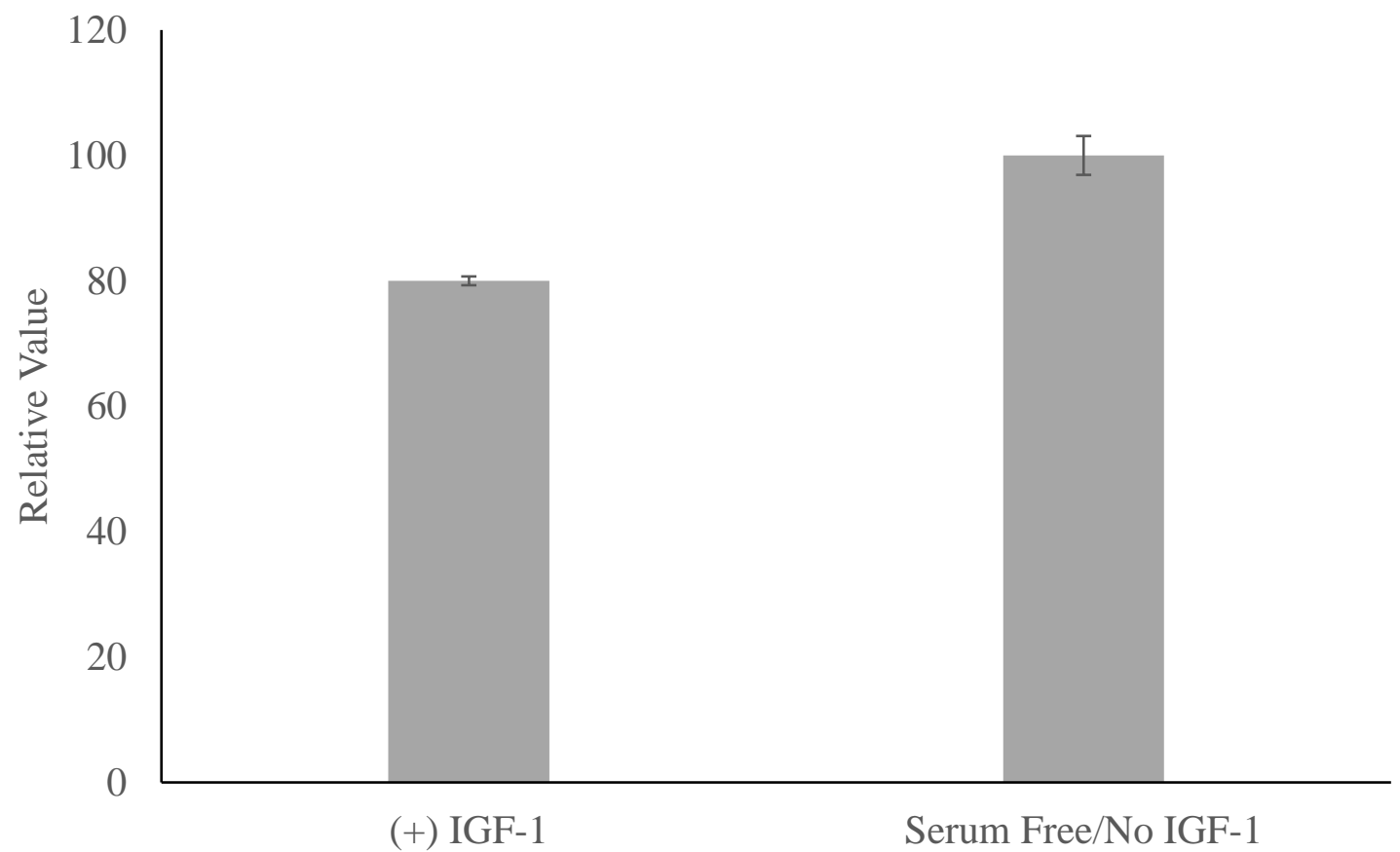

Figure 2.15: The effect of IGF-1 stimulation $(100 \mathrm{ng} / \mathrm{mL})$ on cellular proliferation of non-transduced MCF-12A cells. Values represent the percent increase normalized to the serum free/no IGF-1 treatment group. All experiments were conducted in triplicate. Error bars represent the standard error of the mean. 
MCF-12A GFP Proliferation

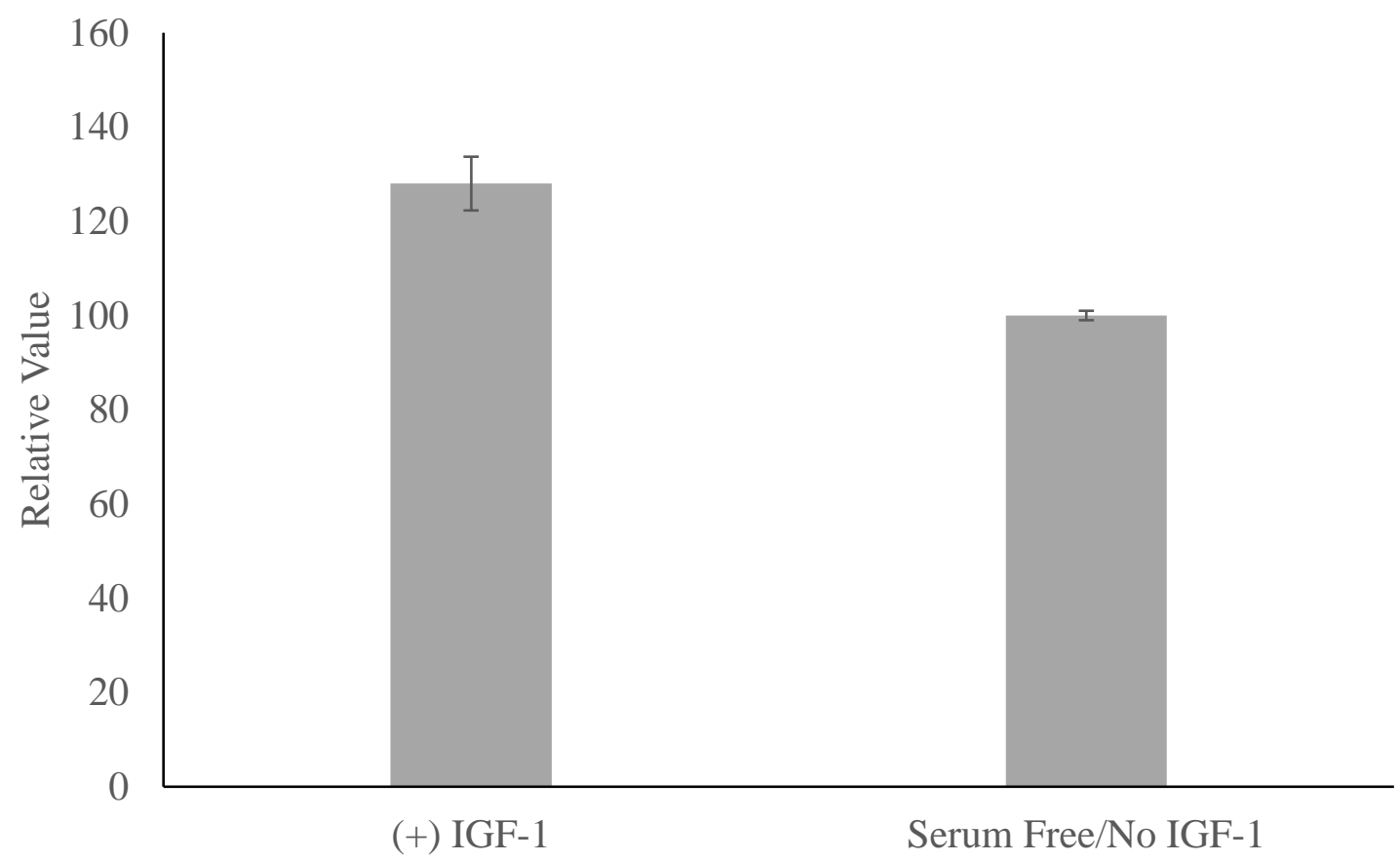

Figure 2.16: The effect of IGF-1 stimulation $(100 \mathrm{ng} / \mathrm{mL})$ on cellular proliferation of MCF-12A cells expressing GFP. Values represent the percent increase normalized to the serum free/no IGF-1 treatment group. All experiments were conducted in triplicate. Error bars represent the standard error of the mean. 
MCF-12A Rin1 Proliferation

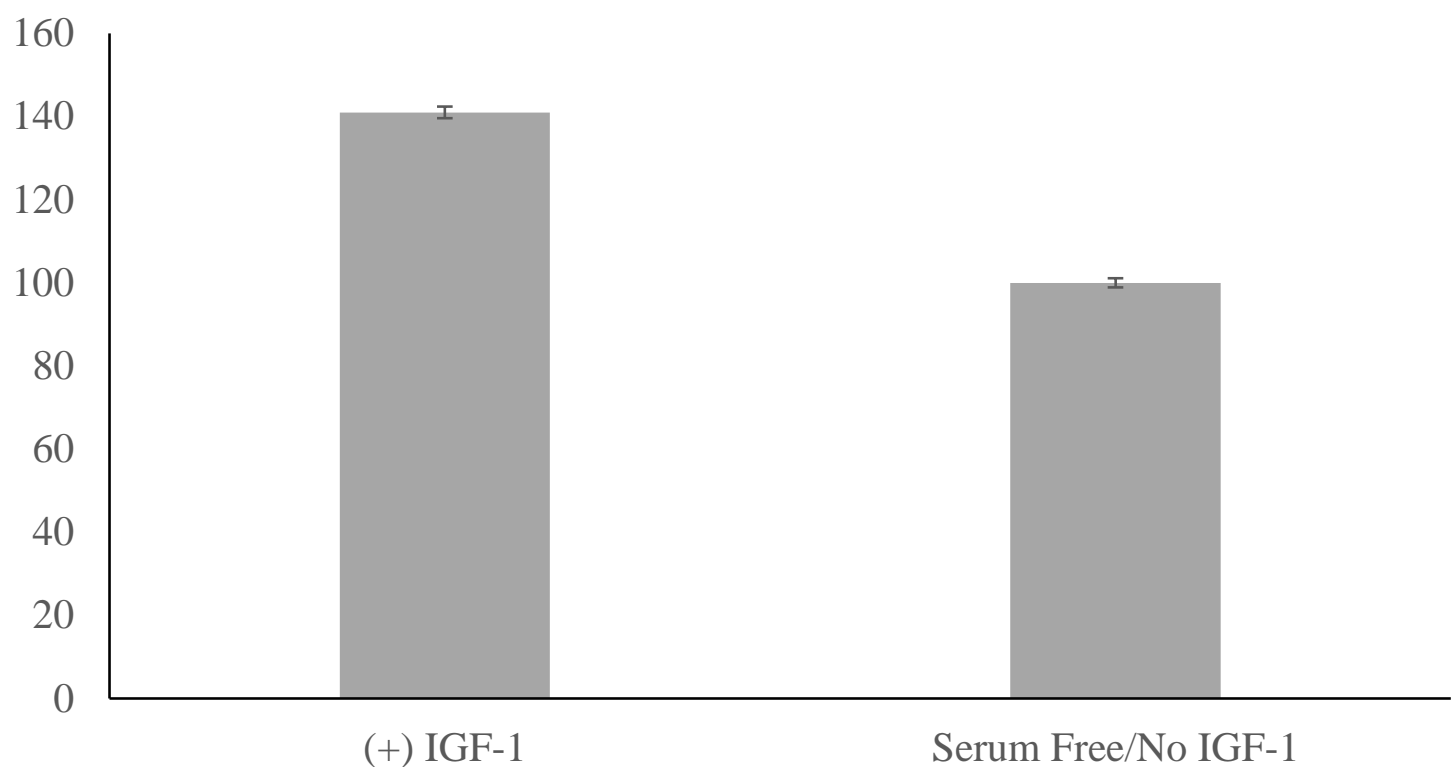

Figure 2.17: The effect of IGF-1 stimulation $(100 \mathrm{ng} / \mathrm{mL})$ on cellular proliferation of MCF-12A cells expressing Rin1. Values represent the percent increase normalized to the serum free/no IGF-1 treatment group. All experiments were conducted in triplicate. Error bars represent the standard error of the mean. 


\begin{tabular}{|l|c|}
\hline \multicolumn{2}{|c|}{ MCF-12A Telomerase Activity } \\
\hline & Average Ct Value \pm Standard Error \\
\hline Telomerase positive control & $25.56 \pm 0.11$ \\
\hline Telomerase negative control & $25.70 \pm 0.15$ \\
\hline GFP (+) IGF-1 & $25.92 \pm 0.22$ \\
\hline GFP (-) IGF-1 & $25.57 \pm 0.02$ \\
\hline Rin1 (+) IGF-1 & $25.76 \pm 0.12$ \\
\hline Rin1 (-) IGF-1 & $25.74 \pm 0.14$ \\
\hline Buffer & $26.06 \pm 0.18$ \\
\hline
\end{tabular}

$25.76-25.92=-0.16$
$2^{-(-0.16)}=1.12$
1.12 fold increase in telomerase activity for cells
expressing Rin1 as compared to cells expressing GFP.

Table 2.4: Cycle threshold values $(\mathrm{Ct})$ for the qPCR products of telomerase activity in MCF-12A cell lines following stimulation with $100 \mathrm{ng} / \mathrm{mL}$ of IGF-1 (+) for 24 hours or no stimulation (-). All experiments were conducted in duplicate. 


\begin{tabular}{|c|c|c|c|}
\hline \multicolumn{4}{|c|}{ MCF-12A Telomerase Gene Expression } \\
\hline & GAPDH & Telomerase & \multirow{2}{*}{$\begin{array}{c}\Delta \mathrm{Ct} \\
\text { (Telomerase- } \\
\text { GAPDH) }\end{array}$} \\
\hline & $\begin{array}{c}\text { Average Ct Value } \pm \\
\text { Standard Error }\end{array}$ & $\begin{array}{c}\text { Average Ct Value } \pm \\
\text { Standard Error }\end{array}$ & \\
\hline GFP (+) & $18.13 \pm 0.08$ & $22.36 \pm 2.41$ & 4.23 \\
\hline GFP (-) & $18.72 \pm 0.37$ & $24.70 \pm 0.08$ & 5.98 \\
\hline $\operatorname{Rin} 1(+)$ & $18.06 \pm 0.05$ & $23.64 \pm 1.23$ & 5.58 \\
\hline Rin1 (-) & $18.18 \pm 0.35$ & $24.89 \pm 0.35$ & 6.71 \\
\hline \multirow[t]{2}{*}{ Buffer } & $33.33 \pm 0.71$ & $31.90 \pm 0.85$ & -1.43 \\
\hline & $\begin{array}{l}8-4.23=1.35 \\
.35=0.39 \\
.39=2.55 \\
5 \text { fold decrease in telo } \\
\text { pressing Rin } 1 \text { as com }\end{array}$ & $\begin{array}{l}\text { erase gene expression } \\
\text { ed to cells expressing }\end{array}$ & cells \\
\hline
\end{tabular}

Table 2.5: Cycle threshold values $(\mathrm{Ct})$ for the RT-qPCR of telomerase gene expression in MCF-12A cell lines following stimulation with $100 \mathrm{ng} / \mathrm{mL}$ of IGF-1 (+) for 24 hours or no stimulation (-). All experiments were conducted in duplicate. 


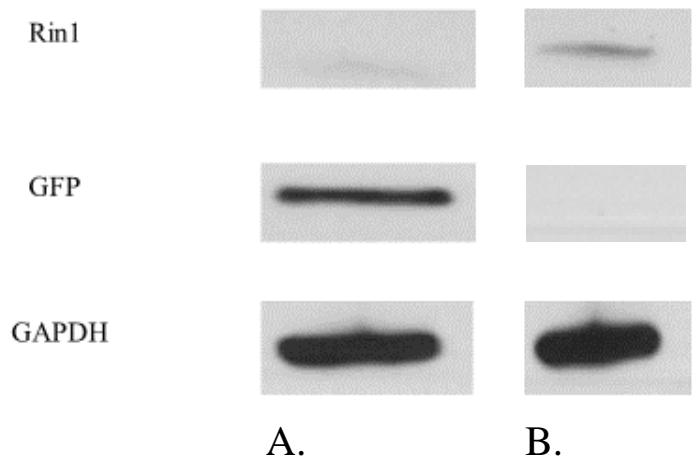

Figure 2.18: Western blot image of the A) MCF7 GFP cell line expressing GFP and endogenous levels of Rin1. B) MCF7 cell line overexpressing Rin1.

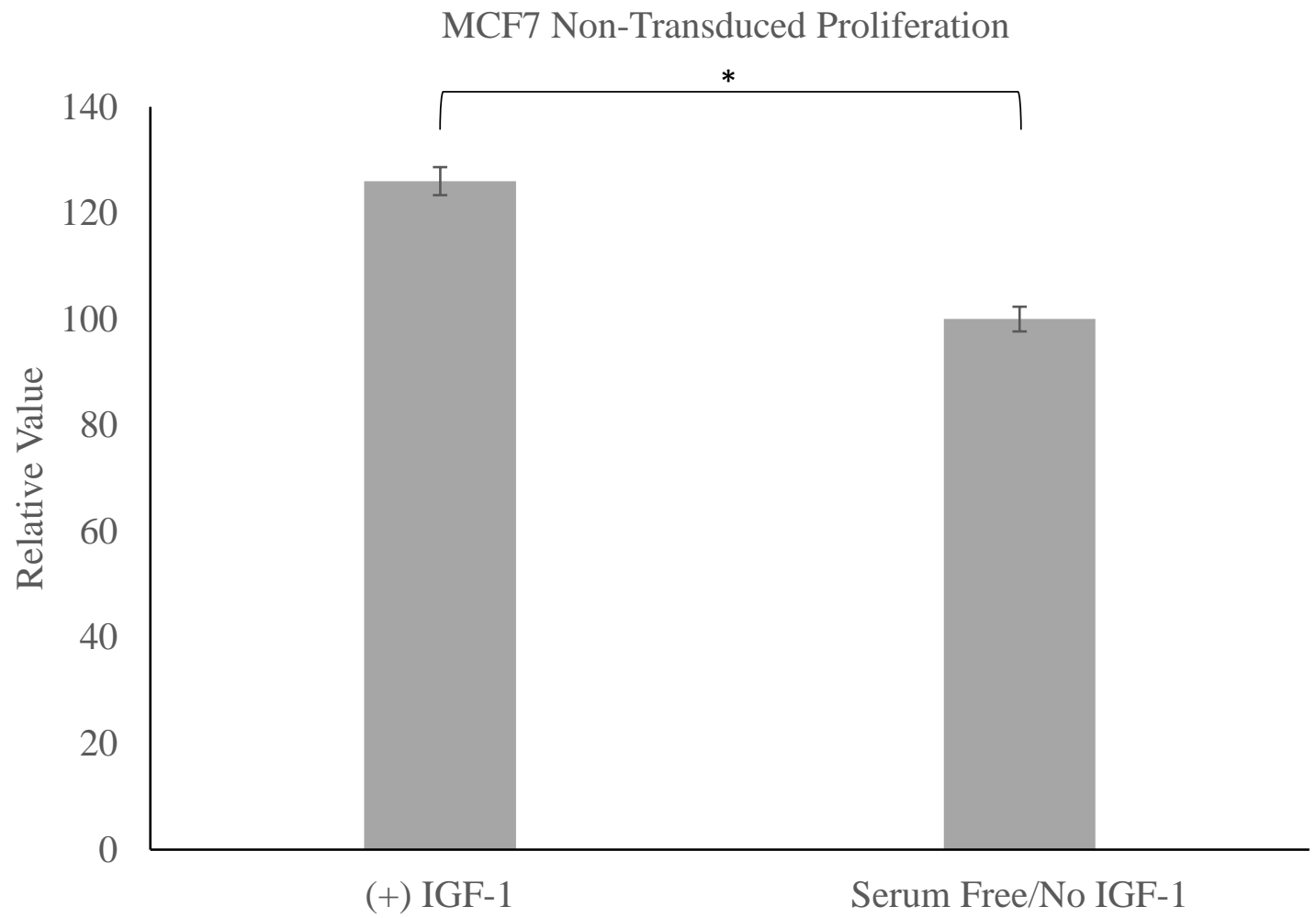

Figure 2.19: The effect of IGF-1 stimulation $(100 \mathrm{ng} / \mathrm{mL})$ on cellular proliferation of non-transduced MCF7 cells. Values represent the percent increase normalized to the serum free/no IGF-1 treatment group. All experiments were conducted in triplicate. Error bars represent the standard error of the mean. 


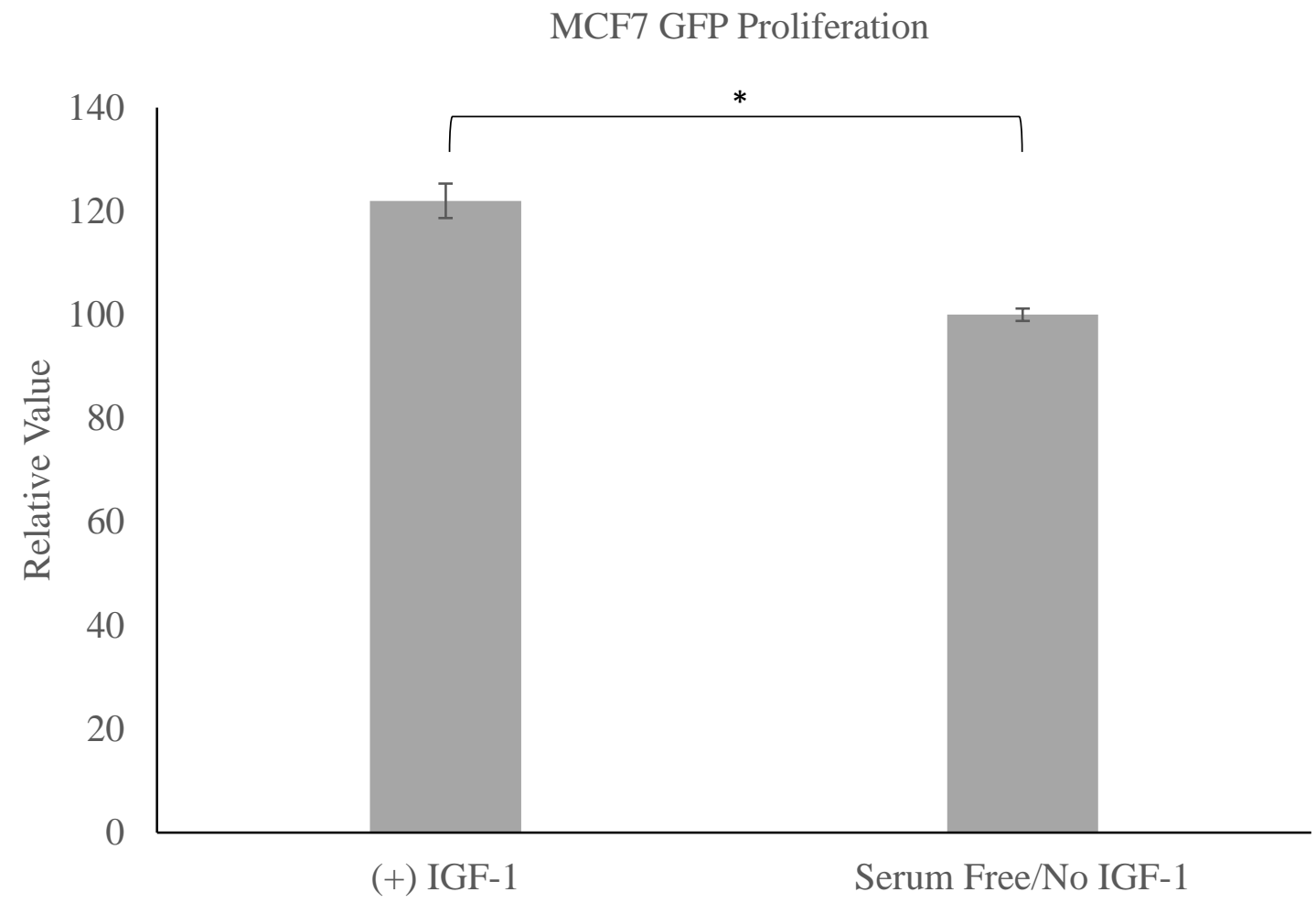

Figure 2.20: The effect of IGF-1 stimulation $(100 \mathrm{ng} / \mathrm{mL})$ on cellular proliferation of MCF7 cells expressing GFP. Values represent the percent increase normalized to the serum free/no IGF-1 treatment group. All experiments were conducted in triplicate. Error bars represent the standard error of the mean. 


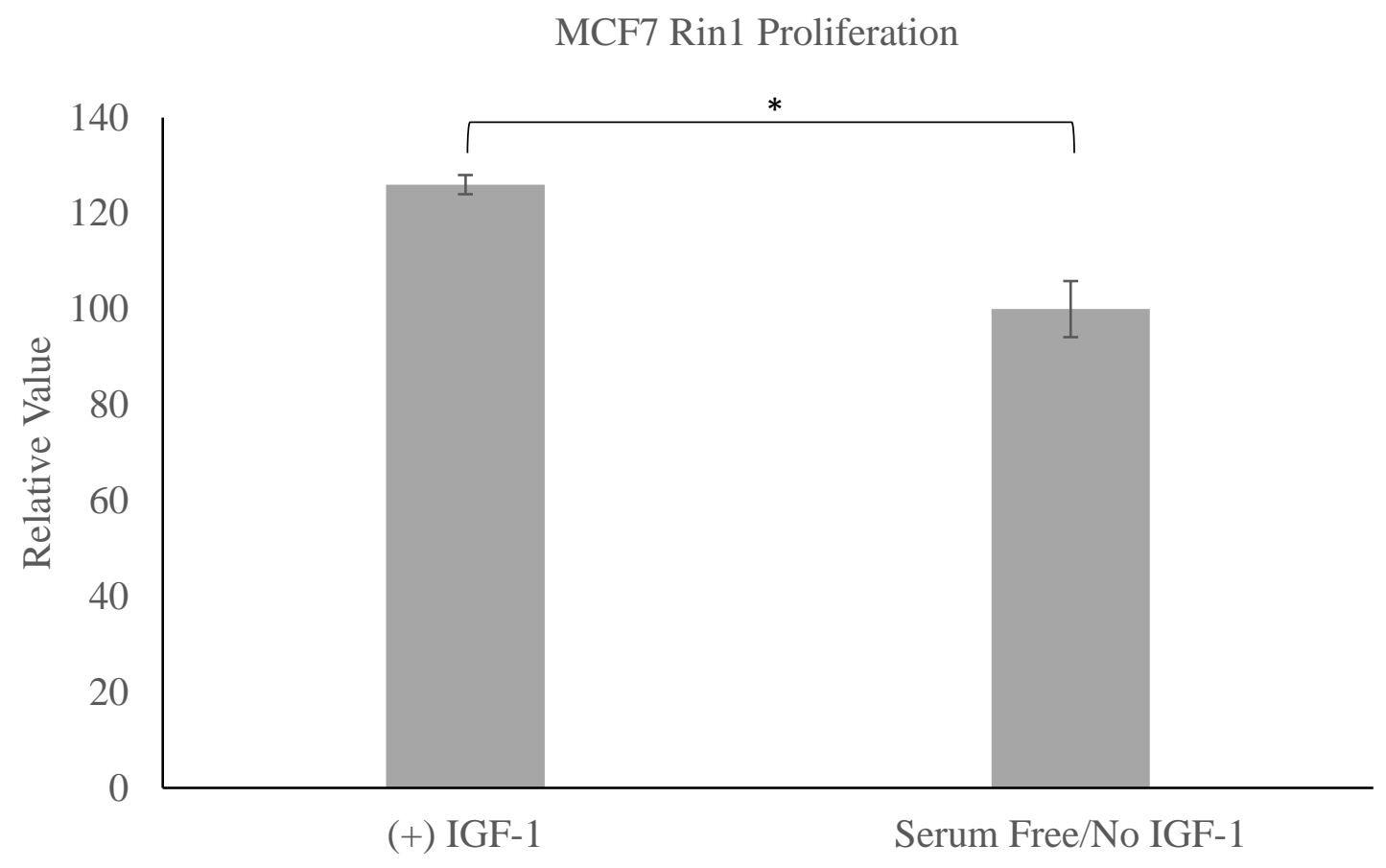

Figure 2.21: The effect of IGF-1 stimulation $(100 \mathrm{ng} / \mathrm{mL})$ on cellular proliferation of MCF7 cells expressing Rin1. Values represent the percent increase normalized to the serum free/no IGF-1 treatment group. All experiments were conducted in triplicate. Error bars represent the standard error of the mean. 


\begin{tabular}{|c|c|}
\hline \multicolumn{2}{|c|}{ MCF7 Telomerase Activity } \\
\hline & Average Ct Value \pm Standard Error \\
\hline Telomerase positive control & $24.53 \pm 0.29$ \\
\hline Telomerase negative control & $25.49 \pm 0.08$ \\
\hline GFP (+) IGF-1 & $24.50 \pm 0.26$ \\
\hline GFP (-) IGF-1 & $24.97 \pm 0.15$ \\
\hline Rin1 (+) IGF-1 & $24.63 \pm 0.04$ \\
\hline Rin1 (-) IGF-1 & $25.46 \pm 0.33$ \\
\hline Buffer & $25.70 \pm 0.16$ \\
\hline $\begin{array}{l}24.63-24.50=0.1 \\
2^{-0.13}=0.91 \\
1 / 0.91=1.09 \\
1.09 \text { fold decreas } \\
\text { expressing Rin } 1\end{array}$ & $\begin{array}{l}\text { lomerase activity for cells } \\
\text { pared to cells expressing GFP. }\end{array}$ \\
\hline
\end{tabular}

Table 2.6: Cycle threshold values $(\mathrm{Ct})$ for the $\mathrm{qPCR}$ products of telomerase activity in MCF7 cell lines following stimulation with $100 \mathrm{ng} / \mathrm{mL}$ of IGF-1 (+) for 24 hours or no stimulation (-). All experiments were conducted in duplicate. 


\begin{tabular}{|c|c|c|c|}
\hline \multicolumn{4}{|c|}{ MCF7 Telomerase Gene Expression } \\
\hline & GAPDH & Telomerase & \multirow{2}{*}{$\begin{array}{c}\Delta \mathrm{Ct} \\
\text { (Telomerase- } \\
\text { GAPDH) }\end{array}$} \\
\hline & $\begin{array}{l}\text { Average Ct Value } \pm \\
\text { Standard Error }\end{array}$ & $\begin{array}{l}\text { Average Ct Value } \pm \\
\text { Standard Error }\end{array}$ & \\
\hline GFP $(+)$ & $16.44 \pm 0.44$ & $26.98 \pm 0.48$ & 10.54 \\
\hline GFP (-) & $17.04 \pm 0.10$ & $27.63 \pm 0.03$ & 10.59 \\
\hline $\operatorname{Rin} 1(+)$ & $16.53 \pm 0.00$ & $27.40 \pm 0.13$ & 10.87 \\
\hline $\operatorname{Rin} 1(-)$ & $17.61 \pm 0.57$ & $28.92 \pm 0.12$ & 11.31 \\
\hline \multirow[t]{2}{*}{ Buffer } & $36.68 \pm 1.13$ & $34.40 \pm 0.56$ & -2.28 \\
\hline & $\begin{array}{l}.87-10.54=0.33 \\
0.33=0.79 \\
0.79=1.26 \\
26 \text { fold decrease in tel } \\
\text { pressing Rin } 1 \text { as com }\end{array}$ & erase gene expression & cells \\
\hline
\end{tabular}

Table 2.7: Cycle threshold values (Ct) for the RT-qPCR of telomerase gene expression in MCF7 cell lines following stimulation with $100 \mathrm{ng} / \mathrm{mL}$ of IGF-1 (+) for 24 hours or no stimulation (-). All experiments were conducted in duplicate. 


\begin{tabular}{|c|c|c|c|}
\hline \multicolumn{3}{|c|}{ Comparison of the Effect of Rin1 Expression in Breast Cell Lines } \\
\hline & MDA-MB 231 & MCF-12A & MCF7 \\
\hline $\begin{array}{c}\text { Cellular Proliferation } \\
\text { (percent change) }\end{array}$ & $(18 \%)$ & + & + \\
\hline $\begin{array}{c}\text { Telomerase Gene } \\
\text { Expression }\end{array}$ & - & & $(26 \%)$ \\
\hline $\begin{array}{c}\text { (fold change) } \\
\text { Telomerase Activity }\end{array}$ & $(1.09)$ & - & - \\
\hline (fold change) & $(1.56)$ & $(1.12)$ & $(1.26)$ \\
\hline
\end{tabular}

Table 2.8: The effect of Rin 1 expression on cellular proliferation, telomerase gene expression, and telomerase activity in different breast cell lines. 


\section{CHAPTER 3}

\section{The Effect of Rin1 Constructs on Telomerase Protein Expression and Activity in Breast Cancer Cells}

\subsection{Introduction}

Telomeres are the regions of DNA that exist at the very tips or ends of linear chromosomes in eukaryotic cells. Recent evidence suggests that the telomere regions of chromosomes play vital roles in regulating normal cellular processes such as proliferation, aging, and senescence/apoptosis (Ramlee et al., 2016). However, a large body of research also implicates telomeres in the unlimited replicative capacity of many types of cancer cells. The telomeric regions of chromosomes set a replicative limit on the number of cell divisions a normal cell can undergo before the induction of senescence and/or apoptosis occurs (Chung Low and Tergaonkar, 2013). The finite replicative capacity, which is approximately 50 to 70 rounds of cell division for most normal somatic cells, is largely due to the progressive loss of telomeric DNA with each round of cell division (Zvereva et al., 2010). The loss of telomeric DNA that accompanies each cycle of cell division is a consequence of incomplete DNA replication at the telomeres.

There are however certain populations of somatic stem cells as well as germ line stem cells that can prevent the gradual loss of DNA from their telomeres through the activity of an enzyme known as telomerase. Telomerase is only expressed and active in a relatively small population of somatic stem cells where the depletion of telomeric DNA and resulting onset of senescence or apoptosis would interfere with the normal functioning of specific tissues (Chung Low and Tergaonkar, 2013). On the other hand, the immortal replicative phenotype that is characteristic of the vast majority of cancer cells is attributed to high 
levels of telomerase activity. In fact, roughly $85 \%$ of all cancers display telomerase activity and the activation of telomerase activity in these cells is a key step in the tumorigenic process (Zhu et al., 2010). Consequently, the selective inhibition of telomerase activity in cancer cells has emerged as an attractive therapeutic target (Holysz et al., 2013; Buseman et al., 2012). A better understanding of telomerase structure, function, and regulation will shed light on the importance of this enzyme in cancer biology.

The telomere regions of vertebrate chromosomes are defined by a distinct structure that distinguishes telomeres from other areas of chromosomal DNA. In humans, a highly repetitive sequence of TTAGGG approximately 5 to $15 \mathrm{~kb}$ in length is tightly associated with a complex of six telomeric DNA binding proteins termed a shelterin complex (Stewart et al., 2012). The majority of telomeric DNA is double stranded with the exception of a guanine rich 3' overhang of single stranded DNA roughly 100 nucleotides in length. The looping of this single stranded DNA into the double stranded regions produces a structure known as a T-loop. The shelterin complex, which assists in the formation of T-loops of telomeric DNA, functions as a protective cap that prevents the activation of cellular DNA damage responses that would otherwise recognize the telomeres as double stranded DNA breaks (Stewart et al., 2012; Buseman et al., 2012). The major role of telomeres is to maintain genomic stability by acting as a buffer for the gradual erosion of DNA that accompanies each cycle of cell division. Once telomeric DNA has shortened beyond a particular threshold, genomic instability as a result of chromosomal fusions and translocations will typically induce senescence followed by apoptosis (Buseman et al, 2012). The mechanism is believed to be a normal part of the aging process. 
The shelterin protein complex is critical for maintaining genomic stability at the telomeres and consists of the following six proteins: TRF1, TRF2, TIN2, TPP1, POT1, and RAP1 (Stewart et al., 2012). TRF1 and TRF2 (telomeric repeat factor-binding protein) each form separate homodimers that bind to the double stranded DNA regions of telomeres. Both TRF1 and TRF2 act as negative regulators of telomere length and are linked to each other by the TIN2 linker protein (Walker et al., 2012). The POT1 (protection of telomeres) binds to the 3' single stranded guanine rich overhang and mainly functions to inhibit the activation of DNA damage response pathways at the telomeres. The POT1 is connected to the other shelterin complex proteins through the linker protein TPP1 which binds to both POT1 and TIN2 (Heidenreich and Kumar, 2017). The RAP1 is a small protein that binds to TRF2 and aids TRF2 in preventing non-homologous end joining and chromosomal fusions (Stewart et al., 2012). The TIN2 maintains the stability of the shelterin complex given its interactions with both TRF1 and TRF2 as well as with TPP1-POT1 (Heidenreich and Kumar, 2017). The interaction between POT1 and TPP1 is essential for the regulation of telomerase activity by enhancing the processivity of the telomerase enzyme (Zaug et al., 2010). Besides the recruitment of telomerase to telomeres, TPP1 is also involved in monitoring the length of telomeres and provides feedback regulation to telomerase (Sexton et al., 2014). Phosphorylation of TPP1 at serine residue 111 is important for the cell cycle dependent recruitment and activation of telomerase activity (Zhang et al., 2013). Each shelterin complex protein plays a clear role in telomere maintenance and the loss of any one protein will result in reduced telomere protection (Bandaria et al., 2016; Erdel et al., 2017). 
Normal somatic cells lose approximately 50 nucleotides of telomeric DNA with each cycle of cell division as a result of incomplete DNA replication during the $\mathrm{S}$ phase. Incomplete replication of the lagging daughter strand occurs because of the inability of DNA polymerase to access the very 3' end of the template DNA strand (Zvereva et al., 2010). The replication problem is overcome in certain cells by extension of the 3 ' end of the template DNA strand by the telomerase enzyme. Telomerase is a ribonucleoprotein that functions as a reverse transcriptase by adding sequential repeats of the hexameric sequence TTAGGG to the 3' end of the template DNA strand; and once extended by telomerase, DNA polymerase $\alpha$-primase $(\mathrm{P} \alpha \mathrm{P})$ can then complete replication of the lagging daughter strand (Hockemeyer and Collins, 2015).

The functional telomerase holoenzyme consists of two essential parts: an enzymatic protein component known as TERT that acts as a reverse transcriptase and an RNA template component known as TR (Heidenreich and Kumar, 2017). The assembly of functional telomerase holoenzyme proceeds through distinct stages. The TERT mRNA is first synthesized and processed in the nucleus and then exported to the cytoplasm for translation into protein. Newly synthesized TERT enzyme is then imported back into the nucleus where it is localized to the nucleolus (Hockemeyer and Collins, 2015; Podlevsky and Chen, 2012). Meanwhile, TR precursor RNA is transcribed by RNA polymerase II in the nucleus and is capped on the 5 ' end with trimethylguanosine. Two copies of a protein complex containing dyskerin, NOP10, NHP2, and GAR1 associate with the TR at the H/ACA motif and are required for 3' end modification. The RHAU RNA helicase binds to the 5' end of TR and resolves the G-quadruplex while the TCAB1 protein binds to the $\mathrm{CAB}$ box motif and directs the TR to nuclear Cajal bodies (Hockemeyer and Collins, 2015; 
Podlevsky and Chen, 2012). The TERT is subsequently moved into the Cajal bodies where it assembles with TR to form the final functional telomerase holoenzyme. The active telomerase enzyme will then be recruited to the telomeres at the appropriate time for telomeric DNA synthesis (Hockemeyer and Collins, 2015; Podlevsky and Chen, 2012).

The regulation of telomerase activity occurs mainly at the level of TERT transcription as TERT mRNA synthesis is highly regulated in most somatic cells. The synthesis of TERT mRNA appears to be the rate limiting step in the regulation of telomerase activity as TERT gene transcription is highly repressed in most somatic cells (Zhu et al., 2010). The telomerase RNA template (TR) however is ubiquitously expressed in many cell types (Daniel et al., 2012). The TERT core promoter contains binding sites for several key transcription factors known to regulate TERT transcription- these include: c-Myc, SP1, ER, Ets, AP1, and NF-kB (Zhu et al., 2010). The c-Myc binds to two E-box consensus sequences within the TERT promoter and is strongly linked to activation of TERT transcription. Similarly, SP1 plays a critical role in activating TERT transcription as mutation of SP1 binding sites in the TERT promoter significantly reduces TERT expression (Cifuentes-Rojas and Shippen, 2012). There is evidence that SP1 may in fact interact with c-Myc to promote TERT expression and that SP1 may also potentially interact with another protein known as MCAF-1 to stimulate TERT expression. Two estrogen response elements are located within the TERT promoter upstream of the transcription start site and enhance TERT expression when bound by ER $\alpha$ (Daniel et al., 2012).

On the other hand, TERT expression is negatively regulated by the binding of tumor suppressor proteins such as the Wilm's tumor-1 (WT1) protein. Additionally, p53 expression acts to inhibit TERT transcription. The p53 tumor suppressor protein has been 
shown to interact with both human telomerase associated protein 1 (hTEP1) and SP1 to inhibit TERT promoter activity (Lu et al., 2013). The overexpression of TERT transcriptional activators such as c-Myc and the loss of tumor suppressor proteins such as p53 or $\mathrm{Rb}$ are common mechanisms in the malignant transformation of cells (Zhu et al., 2010).

Telomerase activity can also be regulated to a lesser extent by post-translational modifications of the catalytic TERT subunit such as phosphorylation and ubiquitination. Multiple protein kinases like c-Abl, protein kinase $\mathrm{B}$, and protein kinase $\mathrm{C}$ are able to influence telomerase activity (Wojtyla et al., 2010). Tyrosine phosphorylation of TERT by c-Abl tends to reduce TERT activity while phosphorylation of serine/threonine residues by PKB (Akt) tends to stimulate TERT activity. Protein phosphatase 2A has been shown to reduce telomerase activity in specific cell types as TERT phosphorylation is necessary for its import into the nucleus. The TERT stability and half-life in the cytoplasm is controlled by E3 ubiquitin ligases that can target TERT for proteolytic degradation and thus prevent its entry into the nucleus (Cifuentes-Rojas and Shippen, 2012). Several splice variants of human TERT are known to exist each with differing levels of activity. The hTERT $\alpha$ splice variant contains a 183 base pair deletion that results in reduced telomerase activity and thus functions as a dominant negative inhibitor of telomerase activity (Cifuentes-Rojas and Shippen, 2012).

Epigenetic regulation of the TERT promoter figures prominently for TERT expression as the TERT promoter is located within a region of highly condensed chromatin (CifuentesRojas and Shippen, 2012). Core histones within the TERT promoter are usually hypoacetylated and this contributes to the repression of TERT expression observed in most 
somatic cells. $\mathrm{CpG}$ island methylation is also commonly associated with the silencing of TERT expression. Conversely, methylation of lysine 9 of histone 3 (H3K9) increases TERT expression as does the hyperacetylation of other core histones through the inhibition of histone deacetylase complexes (HDACs) (Zhu et al., 2010). Several transcription factors are known epigenetically regulate TERT expression through the recruitment of histone acetyltransferases (HATs) or HDACs to the TERT promoter (Lu et al., 2013).

Telomerase expression and activity can be modulated by various signal transduction pathways. The Wnt/ $\beta$-catenin pathway is widely known for its important role during embryonic development, however it has also been implicated in tumor formation. A positive feedback mechanism exists in which $\beta$-catenin can upregulate TERT expression through its interaction with the TERT promoter. The TERT subsequently forms a complex with the BRG1 Wnt transcription factor to stimulate expression of both cyclin D and cMyc (Wu et al., 2013). The c-Myc is a well documented activator of TERT expression. Inflammation is commonly associated with many different cancers and NF- $\kappa \mathrm{B}$ is a primary regulator of chronic inflammation linked with tumorigenesis and cancer progression. Recent evidence suggests that a reciprocal relationship may exist between NF- $\mathrm{kB}$ activity and TERT expression. NF- $\mathrm{kB}$ can bind upstream of the TERT transcription start site to stimulate TERT expression. The TERT, on the other hand, can reinforce NF- $\kappa B$ activity by binding to the p65 subunit of NF- $\mathrm{kB}$ to enhance transcription of inflammatory genes such as IL-6 and TNF- $\alpha$ (Ghosh et al., 2012). Additionally, the RAP1 shelterin complex protein is a key regulator of NF-KB activity (Martinez and Blasco, 2011).

Other signal transduction pathways such as the PI3K/Akt and MAPK pathways also contribute significantly to the regulation of TERT expression and activity. The Akt- 
mediated phosphorylation of specific effector proteins promotes the proteolytic degradation of p53 and the c-Myc competitor protein MAD1 (Peek and Tollesfbol, 2016). Additionally, the PI3K/Akt pathway has been tied to NF- $\mathrm{KB}$ activation as well as the inhibition of TGF- $\beta$ signaling through Jab1 activation and SMAD4 degradation (Daniel et al., 2012). The TGF- $\beta$ signaling pathway is an important inhibitory pathway that mediates cell growth, differentiation, and proliferation. TGF- $\beta$ signaling can suppress c-Myc expression but is susceptible to inhibition by estrogen (Peek and Tollefsbol, 2016). Heeg et al. (2010) report that EGFR overexpression in OKF6 oral-esophageal cells enhances TERT transcription via the Hif1- $\alpha$ transcription factor and directly stimulates telomerase activity through an Akt-dependent phosphorylation of TERT. Similarly, estradiol (E2) is reported to increase telomerase activity in endometrial cancer cells through MAPK induction of TERT transcription. Inhibition of either MEK or ERK resulted in decreased luciferase activity from a reporter plasmid containing the TERT promoter following treatment with E2 (Zhou et al., 2013).

In summary, abnormal telomerase expression and activity is a defining hallmark observed in many different cancers. The immortal replicative phenotype conferred by the enzyme is critical to the survival and proliferation of malignant cancer cells. The regulation of telomerase activity occurs primarily at the level of transcription and typically becomes disordered during the tumorigenic process. The fact that the enzyme is not expressed in most normal somatic cells makes selective telomerase inhibition an attractive chemotherapeutic target. Further research into the oncogenic properties of telomerase is needed to reveal novel therapeutic approaches. 


\subsection{Results}

Lentiviral vectors encoding Rin1 Y561F and Rin1 delta were designed using the VectorBuilder online platform (https://en.vectorbuilder.com/). These lentiviral vectors (Figure 3.1) were used in the creation of the Rin1 Y561F and Rin1 delta MDA-MB 231 breast cancer cell lines as described in 3.4, Creation of Cell Lines. Light micrograph images of the MDA-MB 231 cell lines overexpressing the Rin1 Y561F or Rin1 delta constructs reveal similarities in morphology and growth rate (Figures 3.2 and 3.3, B.) The cells of each cell line displayed a flattened, spindle type shape and grew fairly evenly across the surface of the culture dish. The growth rate of each was similar to that of MDA-MB 231 cells expressing GFP. Additionally, confocal imagery indicates a roughly uniform distribution of Rin1 Y561F and Rin1 delta throughout the cytoplasm of these cells (Figures 3.2 and 3.3, A.) while Western blotting analysis reveals strong expression of the different Rin1 constructs (Figures 3.4 and 3.5).

An experiment to determine the effect of different IGF-1 concentrations on the cellular proliferation of MDA-MB 231 cells was performed using IGF-1 concentrations of 20, 60, and $100 \mathrm{ng} / \mathrm{mL}$. The MDA-MB 231 cell lines overexpressing Rin1 Y561F and Rin1 delta each displayed an increase in cellular proliferation for every IGF-1 concentration tested when compared to the corresponding untreated $(0 \mathrm{ng} / \mathrm{mL})$ control cells (Figure 3.11). The same result was observed for the GFP control cell line (Figure 3.11). The effect of IGF-1 concentration on proliferation was statistically significant for all cell lines tested $(\mathrm{p}<0.05$, ANOVA), and stimulation with as little as $20 \mathrm{ng} / \mathrm{mL}$ of IGF-1 resulted in an increase in cellular proliferation as compared to untreated cells (Figure 3.11). The MDA-MB 231 Rin1 Y561F and Rin1 delta cell lines displayed an optimal increase in cellular proliferation when 
stimulated with $100 \mathrm{ng} / \mathrm{mL}$ of IGF-1. All proliferation experiments were conducted in triplicate using a concentration of $2.0 \times 10^{5}$ cells $/ \mathrm{mL}$.

An experiment to determine the effect of IGF-1 concentration on telomerase protein expression in MDA-MB 231 GFP cells was conducted using IGF-1 concentrations of 0 , 10 , and $50 \mathrm{ng} / \mathrm{mL}$. Telomerase protein expression increased as the IGF-1 concentration increased and peaked in response to stimulation with an IGF-1 concentration of $50 \mathrm{ng} / \mathrm{mL}$ for 12 hours (Figure 3.6). An ImageJ analysis of the intensity of telomerase protein expression at $50 \mathrm{ng} / \mathrm{mL}$ of IGF-1 indicates an approximately 13 fold increase over unstimulated cells and a roughly 2 fold increase over cells stimulated with $10 \mathrm{ng} / \mathrm{mL}$ (Figure 3.6). The effect of IGF-1 stimulation on telomerase protein expression was subsequently evaluated in MDA-MB 231 cells expressing Rin1, Rin1 Y561F, and Rin1 delta. The cells were induced with $50 \mathrm{ng} / \mathrm{mL}$ of IGF-1 for 24 hours and the intensity of telomerase protein expression was normalized to that of GFP cells using ImageJ analysis (Figure 3.7). Increases in telomerase protein expression of 3 fold and 2 fold respectively were observed for the MDA-MB 231 Rin1 Y561F and Rin1 delta cell lines, while the Rin1 cell line exhibited a 0.7 fold decrease in telomerase protein expression (Figure 3.7).

In order to study the effects of the amino (R2N) and carboxyl (R3C) terminal regions of Rin1 on signaling through the MAPK pathway, MDA-MB 231 cells expressing these Rin 1 constructs were stimulated with $100 \mathrm{ng} / \mathrm{mL}$ of IGF-1 for 24 hours. Levels of total p44/42 and phospho-p44/42 protein expression were then determined through Western blotting (Figure 3.8) and the ratio of phospho-p44/42 to total p44/42 was calculated by ImageJ analysis (Figure 3.8). A 16 fold increase in the ratio of phospho-p44/42 to total 
p44/42 was observed for the cells expressing the amino terminus of Rin 1 when compared to the cells expressing the carboxyl terminus (Figure 3.8).

A time course experiment to investigate the effect of Rin1 on signaling through the MAPK pathway was performed on the MDA-MB 231 cells expressing GFP, Rin1, the amino (R2N), and the carboxyl (R3C) terminal regions of Rin1. The expression of various MAPK pathway proteins and other associated proteins as well as their phosphorylation status was determined by Western blotting following IGF-1 stimulation with $25 \mathrm{ng} / \mathrm{mL}$ for either 0, 5, or 10 minutes (Figures 3.9 and 3.10). The R3C cell line exhibited reduced levels of $\mathrm{p} 44 / 42$ phosphorylation over the 10 minute time period when compared to the GFP and R2N cell lines (Figures 3.9, A. and 3.10, A.). The Rin1 cells also display delayed p-44/42 phosphorylation as well as delayed phosphorylation of the Ets2 transcription factor (Figure 3.9, B.). Additionally, low levels of phosphorylated STAT3 and Ets2 transcription factors were observed for the R3C cell line (Figure 3.10, B.).

Telomerase activity was also examined in various MDA-MB 231 breast cancer cell lines expressing different constructs of the Rin1 protein following treatment with IGF-1. These cell lines include the MDA-MB 231 human breast cancer cell lines expressing Rin1 Y561F and Rin1 delta as well as the amino (R2N) and carboxyl (R3C) terminal regions of Rin1. Each cell line was virally transduced and selected for stable Rin1 construct expression as described previously (2.4, Creation of Cell Lines). Telomerase activity following exposure to $100 \mathrm{ng} / \mathrm{mL}$ of IGF-1 for 24 hours was determined by the telomeric repeat amplification protocol (TRAP) assay for each of the MDA-MB 231 cell lines expressing the various Rin1 constructs (Table 3.1). 
The MDA-MB 231 breast cancer cell lines overexpressing Rin1 Y561F and Rin1 delta displayed elevated telomerase activities of approximately 1 fold each over GFP cells when treated with $100 \mathrm{ng} / \mathrm{mL}$ of IGF-1 for 24 hours (Table 3.1). The MDA-MB 231 GFP cells, which express endogenous levels of Rin1, resulted in an average Ct value of 25.94 while the Rin1 Y561F and delta cell lines produced average Ct values of 25.88 and 25.92 respectively (Table 3.1). The corresponding IGF-1 untreated samples for each cell line were also run for comparison (Table 3.1). A known telomerase positive HeLa cell control sample was run as a positive control and telomerase negative controls consisted of a heat inactivated HeLa cell sample as well as a buffer only sample (Table 3.1). In contrast to the Rin1 Y561F and delta cell lines, the MDA-MB 231 cell lines expressing the amino (R2N) and carboxyl (R3C) regions of Rin1 exhibited 1.06 and 1.11 fold reductions in telomerase activity when compared to GFP cells. The MDA-MB 231 cell lines expressing the R2N and $\mathrm{R} 3 \mathrm{C}$ regions of Rin1 resulted in average $\mathrm{Ct}$ values of 26.03 and 26.09 respectively (Table 3.1).

\subsection{Discussion}

Abnormal telomerase protein expression and activity are commonplace for a number of different cancers. The fact that telomerase activity is virtually absent in most normal somatic cells but present in roughly $85 \%$ of all cancers highlights the importance of the enzyme for cancer cell survival and replication. Strategies to inhibit telomerase activity are therefore attractive chemotherapeutic targets. One such strategy is the potential use of the Rin1 protein to modulate signaling through the MAPK pathway and thus suppress telomerase activity. To this end, several Rin1 constructs were expressed in MDA-MB 231 breast cancer cells to investigate the ability of Rin1 to moderate telomerase activity. 
Cells virally transduced to express GFP were used as a control to compare the effect of overexpression of particular Rin1 constructs (Y561F, delta, amino terminus, carboxyl terminus) on telomerase activity for the MDA-MB 231 breast cancer cell line. The GFP cells express endogenous levels of Rin1 and therefore would be most similar to nontransduced cells in terms of Rin 1 expression. However, the GFP cells are virally transduced which permits meaningful comparisons with the cell lines that are virally transduced to express the various Rin 1 constructs. The expression of GFP has no influence on telomerase activity.

As discussed previously (1.3, The MAPK Pathway and Cancer), Rin1 is able to compete strongly with RAF1 for access to activated Ras. Thus, is it possible that Rin1 may act to dampen mitogenic signaling through the MPAK pathway and consequently affect the activity of a downstream MAPK effector such as telomerase. If so, the potential tumor suppressive role of Rin1 would be evidenced in reduced telomerase activity. Conversely, overexpression of the Rin1 Y561F and Rin1 delta constructs should not impede telomerase activity as these Rin1 isoforms do not bind as strongly to activated Ras (1.3, The MAPK Pathway and Cancer). Similarly, it is hypothesized that overexpression of the Rin1 amino terminal region (R2N) should allow for enhanced MAPK signaling given that the R2N region of Rin1 lacks the Ras association (RA) domain. Conversely, the carboxyl terminal region (R3C) of Rin1 contains the Ras association domain and its overexpression should therefore dampen signaling through the MAPK pathway.

The effect of the Rin1 Y561F and Rin1 delta constructs on cellular proliferation in MDA-MB 231 cells underscores the tumorigenic properties of these particular forms of Rin1 when compared to cells that overexpress Rin1 (Figures 3.11 and 2.6). For instance, 
fold increases in proliferation of 1.2, 1.7, and 9.3 were observed respectively for MDAMB 231 cells expressing Rin1 Y561F across the range of IGF-1 concentrations (20, 60, and $100 \mathrm{ng} / \mathrm{mL}$ ) tested when compared to cells expressing Rin1 (Figure 2.6). Similarly, fold increases in proliferation of 1.5, 2.0, and 9.2 were observed respectively for the cells expressing Rin1 delta over the Rin1 cells when stimulated with the same range of IGF-1 concentrations (Figures 3.11 and 2.6). These data, in conjunction with the observable differences in growth patterns and rates as well as increased telomerase activity, point to heightened MAPK signaling in MDA-MB 231 cells expressing the Rin1 Y561F and delta constructs following IGF-1 stimulation- most likely as a consequence of reduced binding to activated Ras. The Rin1 cells consistently displayed the least amount of increase in proliferation over the corresponding unstimulated cells for each of the IGF-1 concentrations examined, probably owing to increased competition with RAF1 for access to activated Ras (Figure 2.6).

The enhanced MAPK signaling in MDA-MB 231 cells expressing the Rin1 Y561F and Rin1 delta constructs is also evident in telomerase protein expression and correlates well with telomerase activity for these cell lines. Stimulation of GFP cells, which express low endogenous levels of Rin1, results in a steady increase in telomerase protein expression over a range $(0,10$, and $50 \mathrm{ng} / \mathrm{mL})$ of IGF-1 concentrations (Figure 3.6). When normalized to telomerase protein expression in GFP cells, there is a clear reduction in telomerase protein expression of 0.7 fold for the MDA-MB 231 cells overexpressing Rin1 versus either Rin1 Y561F or Rin1 delta, that display fold increases of 3 and 2 respectively (Figure 3.7). As a downstream MAPK effector, changes in telomerase protein expression mirror the ability of Rin1 or its constructs to moderate signaling through the pathway. 
When examining the expression and phosphorylation status of key MAPK proteins over time subsequent to IGF-1 stimulation, the ability of Rin1 to attenuate signaling is evident for $\mathrm{p}-44 / 42$ (ERK). There is suppressed phosphorylation of $\mathrm{p}-44 / 42$ over a 10 minute time period for the MDA-MB 231 cells overexpressing Rin1 as compared to the cells expressing the Rin1 amino (R2N) terminus (Figures 3.9, B. and 3.10, A.). Additionally, appreciable phosphorylation of p-44/42 is not evident for the cells expressing the Rin1 carboxyl (R3C) terminus (Figure 3.10, B.). In a separate experiment, a 16 fold increase in the ratio of phosphorylated p-44/42 to total p-44/42 was observed for the Rin1 R2N cells as compared to the $\mathrm{R} 3 \mathrm{C}$ cells (Figure 3.8). Again, the prevalence of the Ras association (RA) domain in the Rin 1 and R3C cells could explain the differences and correlates well with the reduced telomerase activity observed in both the Rin1 and R3C cell lines.

The MDA-MB 231 Rin1 cells exhibit suppressed phosphorylation of the Ets2 transcription factor, which is a key activator of telomerase protein expression (Figure 3.9, B.). A similar reduction in STAT3 phosphorylation is also apparent in these cells as well as in the R3C cells (Figure 3.10, B.). Taken together, the data highlight a potential tumor suppressor role for Rin1 in MDA-MB 231 breast cancer cells. The decrease in telomerase activity observed for the Rin1 and R3C cells (Tables 2.2 and 3.1) is particularly interesting given that the MDA-MB 231 cell line contains a constitutively active mutant form of Ras; therefore, overexpression of Rin1 in these cells appears to effectively counter the heightened signaling through the MAPK pathway.

In summary, telomerase activity in MDA-MB 231 breast cancer cells is modulated by the form of Rin1 that is expressed. While Rin1 Y561F and Rin1 delta do not bind as strongly to activated Ras when compared to Rin1, the deletion of 62 amino acids from Rin1 
delta and the Y561F point mutation appear to affect their ability to bind with activated Ras and thus permit enhanced signaling through the MAPK pathway. The inhibitory effect of Rin1 on telomerase activity may be partially explained by the interaction of its Ras Association (RA) domain with Ras. The RA domain of Rin1 is located in the carboxy terminus of Rin1 and overexpression of the Rin1 carboxy terminal region (R3C) in MDAMB 231 cells reduces telomerase activity by 1.11 fold. Although Rin1 Y561F and Rin1 delta each contain the RA domain, the altered structures of these Rin 1 constructs due to point mutation and deletion prevents efficient binding to active Ras and may therefore allow for enhanced MAPK signaling over that of Rin1 as evidenced by increased telomerase activity.

\subsection{Materials and Methods}

Creation of Cell Lines

The MDA-MB 231 cell lines stably expressing the Rin1 Y561F and Rin1 delta constructs were created using the same protocol as described previously in 2.4, Creation of Cell Lines. Lentiviral plasmid expression vectors for Rin1 Y561F (pLV[Exp]-PuroCMV >hRIN1Y561F) and Rin1delta (pLV[Exp]-Puro-CMV>hRIN1 delta) were designed using the VectorBuilder website (https://en.vectorbuilder.com/) powered by Cyagen Biosciences. The Rin1 gene sequence was mutated to produce the open reading frame sequences for Rin1 Y561F and Rin1 delta. MDA-MB 231 cell lines expressing the amino (R2N) or carboxyl (R3C) terminal regions of Rin1 were generously provided by Wei Zhang, a fellow graduate student from the Barbieri lab at Florida International University. 


\section{Western Blotting}

Western blotting analysis of the MDA-MB $231 \mathrm{R} 2 \mathrm{~N}$ and R3C cell lines to confirm the expression of the Rin1 amino and carboxyl terminal regions, as well as the Rin1 Y561F and Rin1 delta constructs, was performed in the same manner as described previously in 2.4, Western Blotting. Additionally, Western blotting analysis was utilized to determine the effect of the various MDA-MB 231 Rin1 constructs (Rin1, Rin1 Y561F, Rin1 delta, and GFP) on telomerase protein expression following IGF-1 stimulation $(50 \mathrm{ng} / \mathrm{mL})$ for 24 hours. The effect of stimulation with different IGF-1 concentrations $(0,10$, or $50 \mathrm{ng} / \mathrm{mL})$ for 12 hours on telomerase protein expression was performed using MDA-MB 231 GFP cells.

Determination of the cellular mechanism underlying the regulation of telomerase activity by Rin1 was performed in MDA-MB 231 cell lines expressing either GFP, Rin1, the amino terminal (R2N), or the carboxyl terminal (R3C) of Rin1. Briefly, $2.0 \times 10^{5}$ cells for each MDA-MB 231 construct were grown in $1 \mathrm{~mL}$ starvation media for 24 hours. The starvation media for each cell sample was then replaced with $1 \mathrm{~mL}$ of chilled Krebs-Ringer buffer supplemented with $25 \mathrm{ng} / \mathrm{mL}$ of IGF-1. The cell samples were immediately chilled at $4{ }^{\circ} \mathrm{C}$ with rocking for 30 minutes to allow for IGF-1 receptor binding. The IGF-1 induction $(25 \mathrm{ng} / \mathrm{mL})$ was then performed by incubating the cell samples at $37^{\circ} \mathrm{C}$ for either 0,5 , or 10 minutes. The samples were lysed at these respective times and prepared for Western blotting analysis according to the protocol discussed previously in 2.4, Western blotting. Cell samples were assessed for changes in the levels of protein expression over time for key signaling proteins such as p44/42, phospho-p44/42, STAT3, phospho-STAT3, Ets2, and phospho-Ets2. The effect of the Rin1 amino (R2N) and carboxyl (R3C) regions 
on the ratio of phopho-ERK/ERK was also determined by Western blotting. Cells were stimulated with IGF-1 $(100 \mathrm{ng} / \mathrm{mL})$ for 24 hours and then prepared for Western blotting analysis as described previously in 2.4, Western Blotting.

IGF-1 Assay

In order to assess the effect of insulin-like growth factor 1 (IGF-1) concentration on the proliferation of MDA-MB 231 cells, cells expressing GFP, Rin1, Rin1 Y561F, and Rin1 delta were exposed to various concentrations of IGF-1. The cells were initially grown under the same conditions as described in 2.4, MTT Cell Proliferation Assay. The cells were plated on a 12 well plate at a concentration of $2.0 \times 10^{5}$ cells per $\mathrm{mL}$ with one $\mathrm{mL}$ per well and cultured for 24 hours under serum free conditions. After 24 hours, the cells were exposed in triplicate to four different concentrations of IGF-1: $20 \mathrm{ng} / \mathrm{mL}, 60 \mathrm{ng} / \mathrm{mL}$, and $100 \mathrm{ng} / \mathrm{mL}$. A control group of cells was not exposed to IGF-1 (0 ng/mL). Human IGF-1 (20 ug) was purchased from Shenandoah Biotechnology and diluted in sterile, deionized water to a concentration of $1 \mathrm{ng} / \mathrm{uL}$. After 24 hours of exposure to the various IGF-1 concentrations, the effect of the growth factor on cell proliferation was determined by an MTT assay in the same manner as described under the 2.4, MTT Cell Proliferation Assay. An ANOVA was utilized to detect any statistically significant difference in cell proliferation as a function of IGF-1 concentration.

Telomerase Activity Assay

The MDA-MB 231 human breast cancer cells expressing Rin1 Y561F and Rin1delta as well as the amino (R2N) and carboxyl (R3C) terminal regions of Rin1 were serum starved for 24 hours at $37^{\circ} \mathrm{C}$ and $5 \% \mathrm{CO}_{2}$ on a 12 well plate at a density of $1.0 \times 10^{5}$ cells per well in $1 \mathrm{~mL}$ of serum free growth media. Each of the four MDA-MB 231 cell types were plated 
in duplicate. After 24 hours, $100 \mathrm{ng} / \mathrm{mL}$ of IGF-1 was added to half of the total samplesone well per cell type. The other half of the samples did not receive IGF-1 treatment. The samples were then incubated for another 24 hours, after which time the culture media in each well was removed and the cells were subsequently prepared for the telomeric repeat amplification protocol (TRAP) assay as per the manufacturer's instructions and as previously described in 2.4, Telomerase Activity Assay. 


\subsection{References}

Bandaria, J., Qin, P., Berk, V., Chu, S., \& Yildiz, A. (2016). Shelterin protects chromosome ends by compacting telomeric chromatin. Cell, 164(4), 735-746.

Buseman, C. M., Wright, W. E., \& Shay, J. W. (2012). Is telomerase a viable target in cancer? Mutation Research - Fundamental and Molecular Mechanisms of Mutagenesis, 730(1-2), 90-97.

Chung Low, K., \& Tergaonkar, V. (2013). Telomerase: Central regulator of all of the hallmarks of cancer. Trends in Biochemical Sciences, 38(9), 426-434.

Cifuentes-Rojas, C., \& Shippen, D. E. (2012). Telomerase regulation. Mutation Research - Fundamental and Molecular Mechanisms of Mutagenesis, 730(1-2), 20-27.

Daniel, M., Peek, G. W., \& Tollefsbol, T. O. (2012). Regulation of the human catalytic subunit of telomerase (hTERT). Gene, 498(2), 135-146.

Erdel, F., Kratz, K., Willcox, S., Griffith, J. D., Greene, E. C., \& de Lange, T. (2017). Telomere recognition and assembly mechanism of mammalian shelterin. Cell Reports, 18(1), 41-53.

Ghosh, A., Saginc, G., Leow, S. C., Khattar, E., Shin, E. M., Yan, T. D., \& Tergaonkar,

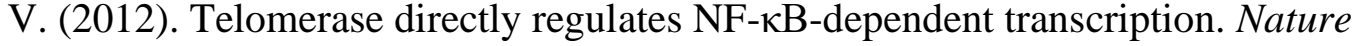
Cell Biology, 14(12), 1270-1281.

Heeg, S., Hirt, N., Queisser, A., Schmieg, H., Thaler, M., Kunert, H., \& Opitz, O. G. (2011). EGFR overexpression induces activation of telomerase via PI3K/AKTmediated phosphorylation and transcriptional regulation through Hifl-alpha in a cellular model of oral-esophageal carcinogenesis. Cancer Science, 102(2), 351-360.

Heidenreich, B., \& Kumar, R. (2017). TERT promoter mutations in telomere biology. Mutation Research-Reviews in Mutation Research, 771, 15-31.

Hockemeyer, D., \& Kathleen Collins. (2015). Control of telomerase action at human telomeres. Nature Structural \& Molecular Biology, 22(11), 848-852.

Holysz, H., Lipinska, N., Paszel-Jaworska, A., \& Rubis, B. (2013). Telomerase as a useful target in cancer fighting - the breast cancer case. Tumor Biology, 34(3), 13711380.

Lu, W., Zhang, Y., Liu, D., Songyang, Z., \& Wan, M. (2013). Telomeres-structure, function, and regulation. Experimental Cell Research, 319(2), 133-141.

Martínez, P., and Blasco, M. A. (2011). Telomeric and extra-telomeric roles for telomerase and the telomere-binding proteins. Nature Reviews Cancer, 11(3), 161176. 
Peek, G. W., \& Tollefsbol, T. O. (2016). Down-regulation of hTERT and cyclin D1 transcription via PI3K/akt and TGF- $\beta$ pathways in MCF-7 cancer cells with PX-866 and raloxifene.Experimental Cell Research, 344(1), 95-102.

Podlevsky, J. D., \& Chen, J. J. (2012). It all comes together at the ends: Telomerase structure, function, and biogenesis. Mutation Research - Fundamental and Molecular Mechanisms of Mutagenesis, 730(1-2), 3-11.

Ramlee, M. K., Wang, J., Toh, W. X., \& Li, S. (2016). Transcription regulation of the human telomerase reverse transcriptase (hTERT) gene. Genes, 7(8), 50.

Sexton, A. N., Regalado, S. G., Lai, C. S., Cost, G. J., O'Neil, C. M., Urnov, F. D., \& Hockemeyer, D. (2014). Genetic and molecular identification of three human TPP1 functions in telomerase action: Recruitment, activation, and homeostasis set point regulation. Genes \& Development, 28(17), 1885-1899.

Stewart, J. A., Chaiken, M. F., Wang, F., \& Price, C. M. (2012). Maintaining the end: Roles of telomere proteins in end-protection, telomere replication and length regulation. Mutation Research - Fundamental and Molecular Mechanisms of Mutagenesis, 730(1-2), 12-19.

Walker, J. R., \& Zhu, X. (2012). Post-translational modifications of TRF1 and TRF2 and their roles in telomere maintenance. Mechanisms of Ageing and Development, 133(6), 421-434.

Wojtyla, A., Gladych, M., \& Rubis, B. (2011). Human telomerase activity regulation. Molecular Biology Reports, 38(5), 3339-3349.

Wu, X., Huang, C., He, X., He, Y., Tian, Y., Zhou, D., \& Li, J. (2013). Feedback regulation of telomerase reverse transcriptase: New insight into the evolving field of telomerase in cancer.Cellular Signalling, 25(12), 2462-2468.

Zaug, A. J., Podell, E. R., Nandakumar, J., \& Cech, T. R. (2010). Functional interaction between telomere protein TPP1 and telomerase. Genes \& Development, 24(6), 613622.

Zhang, Y., Liuh-Yow Chen, Xin Han, Wei Xie, Hyeung Kim, Dong Yang, \& Zhou Songyang. (2013). Phosphorylation of TPP1 regulates cell cycle-dependent telomerase recruitment.Proceedings of the National Academy of Sciences, 110(14), 5457.

Zhou, C., Steplowski, T., Dickens, H.K, Malloy, K.M., Gehrig, P.A., Boggess, J.F., \& Bae-Jump, V.L. (2013). Estrogen induction of telomerase activity through regulation of the mitogen-activated protein kinase (MAPK) dependent pathway in human endometrial cancer cells. PLoS One, 8(2), e55730.

Zhu, J., Zhao, Y., \& Wang, S. (2010). Chromatin and epigenetic regulation of the telomerase reverse transcriptase gene. Protein \& Cell, 1(1), 22-32. 
Zvereva, M., Shcherbakova, D., \& Dontsova, O. (2010). Telomerase: Structure, functions, and activity regulation. Biochemistry (Moscow), 75(13), 1563-1583. 


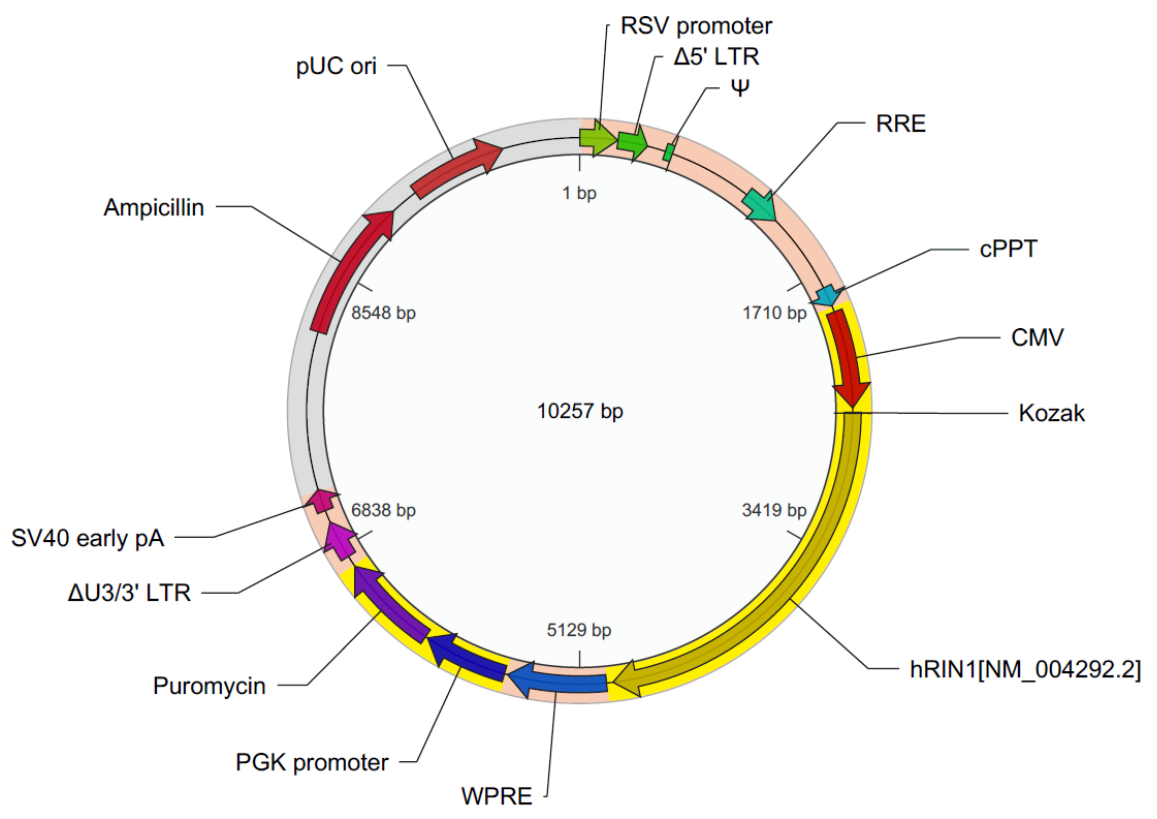

Figure 3.1: Lentiviral vector used to create the MDA-MB 231 cell line expressing Rin1 Y561F. The Rin1 Y561F gene sequence is inserted directly downstream of the CMV promoter. An identical vector with the Rin1 delta gene sequence inserted was used to create the MDA-MB 231 cell line expressing Rin1 delta. Also labeled are the various elements necessary for lentivirus production and cell selection.

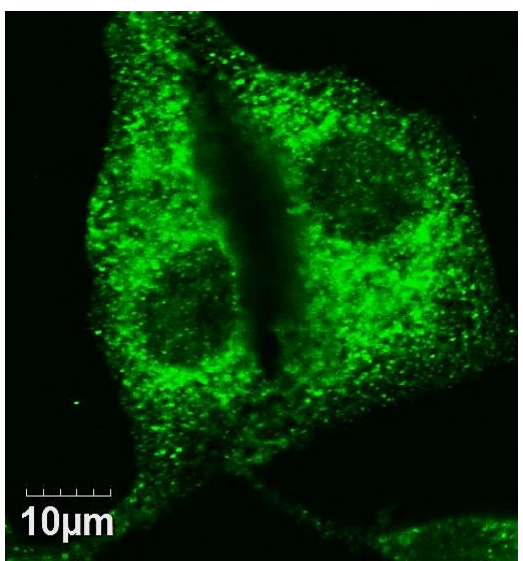

A.

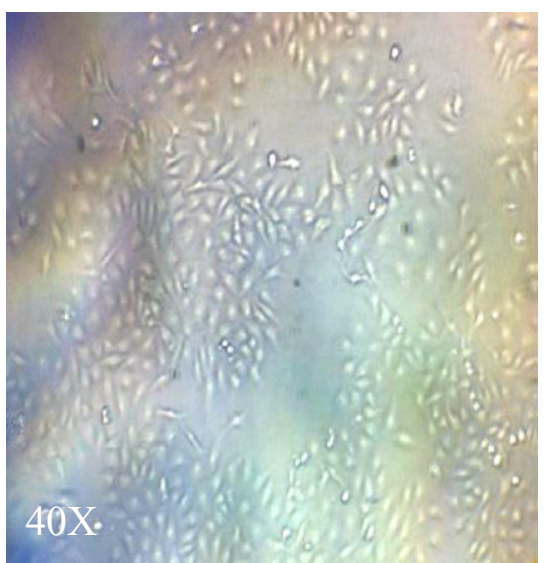

B.

Figure 3.2: A) Confocal micrograph image of MDA-MB 231 cells expressing Rin1 Y561F. Rin1 Y561F tends to localize throughout the cytoplasm. B) Light micrograph image illustrating the even growth pattern of MDA-MB 231 cells expressing Rin1 Y561F. 


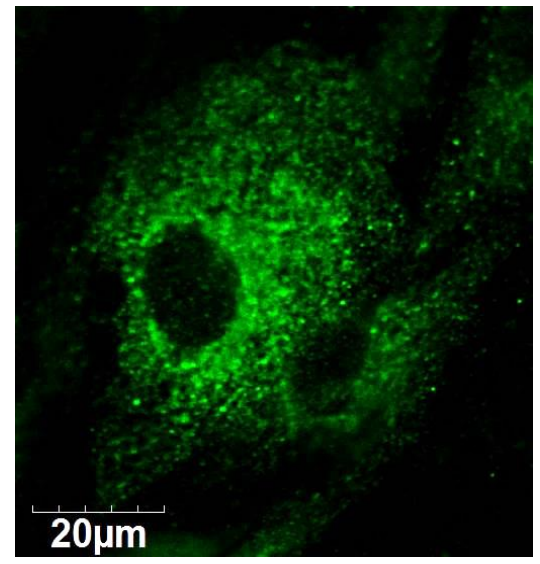

A.

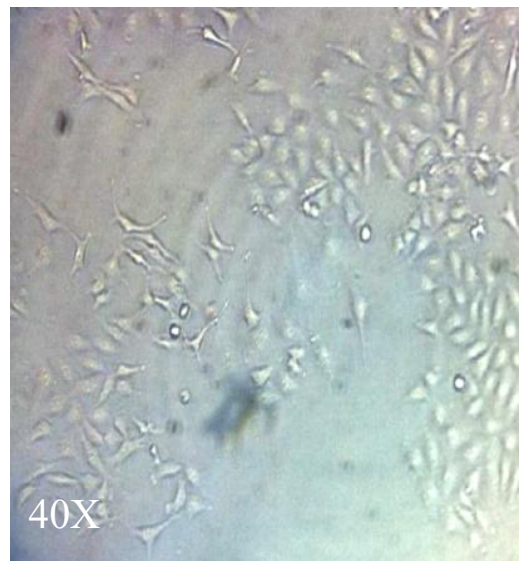

B.

Figure 3.3: A) Confocal micrograph image of MDA-MB 231 cells expressing Rin1 delta. Rin1 delta tends to localize throughout the cytoplasm. B) Light micrograph image illustrating the even growth pattern of MDA-MB 231 cells expressing Rin1 delta.

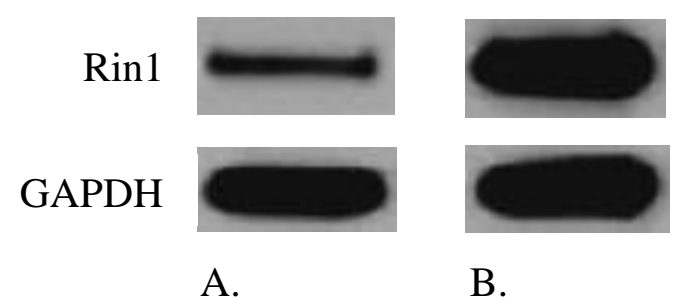

Figure 3.4: Western blot image of the A) MDA-MB 231 Rin1 Y561F cell line expressing Rin1 Y561F. B) MDA-MB 231 Rin delta cell line overexpressing Rin1 delta.

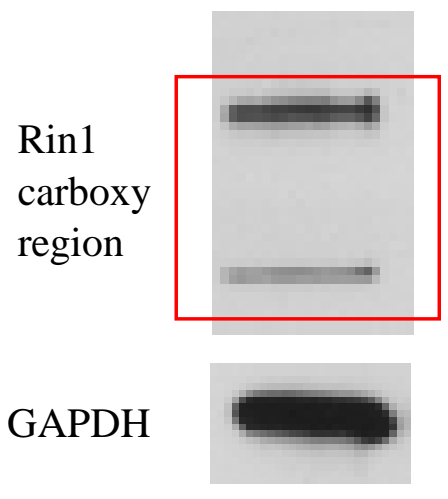

Figure 3.5: Expression of the carboxyl terminal region of Rin1 in the MDA-MB 231 $\mathrm{R} 3 \mathrm{C}$ cell line. 
Telomerase Protein Expression in MDA-MB 231 GFP Cells

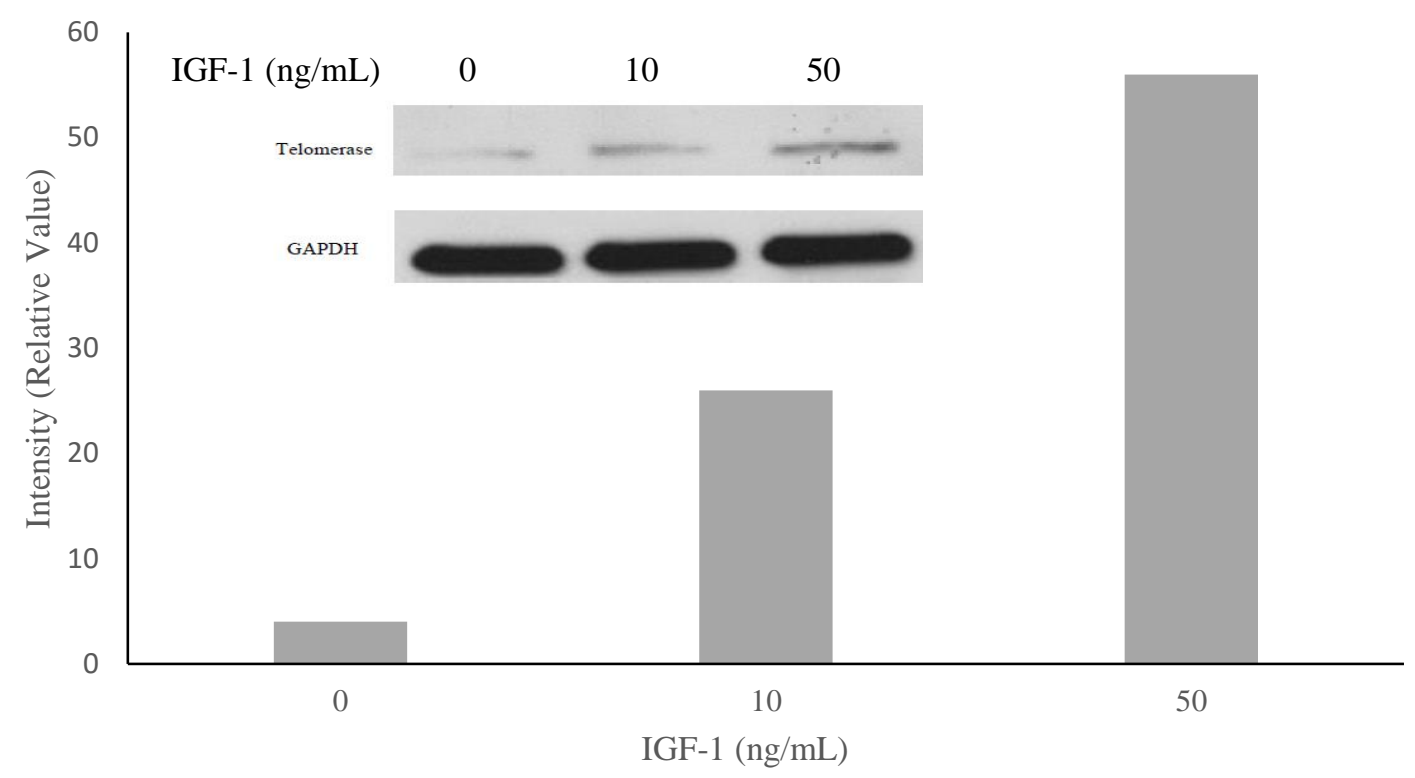

Figure 3.6: Effect of IGF-1 concentration on telomerase protein expression in MDAMB 231 GFP cells. Cells were stimulated with 0 , 10, or $50 \mathrm{ng} / \mathrm{mL}$ of IGF-1 for 12 hours.

Telomerase Protein Expression MDA-MB 231 Cell Lines

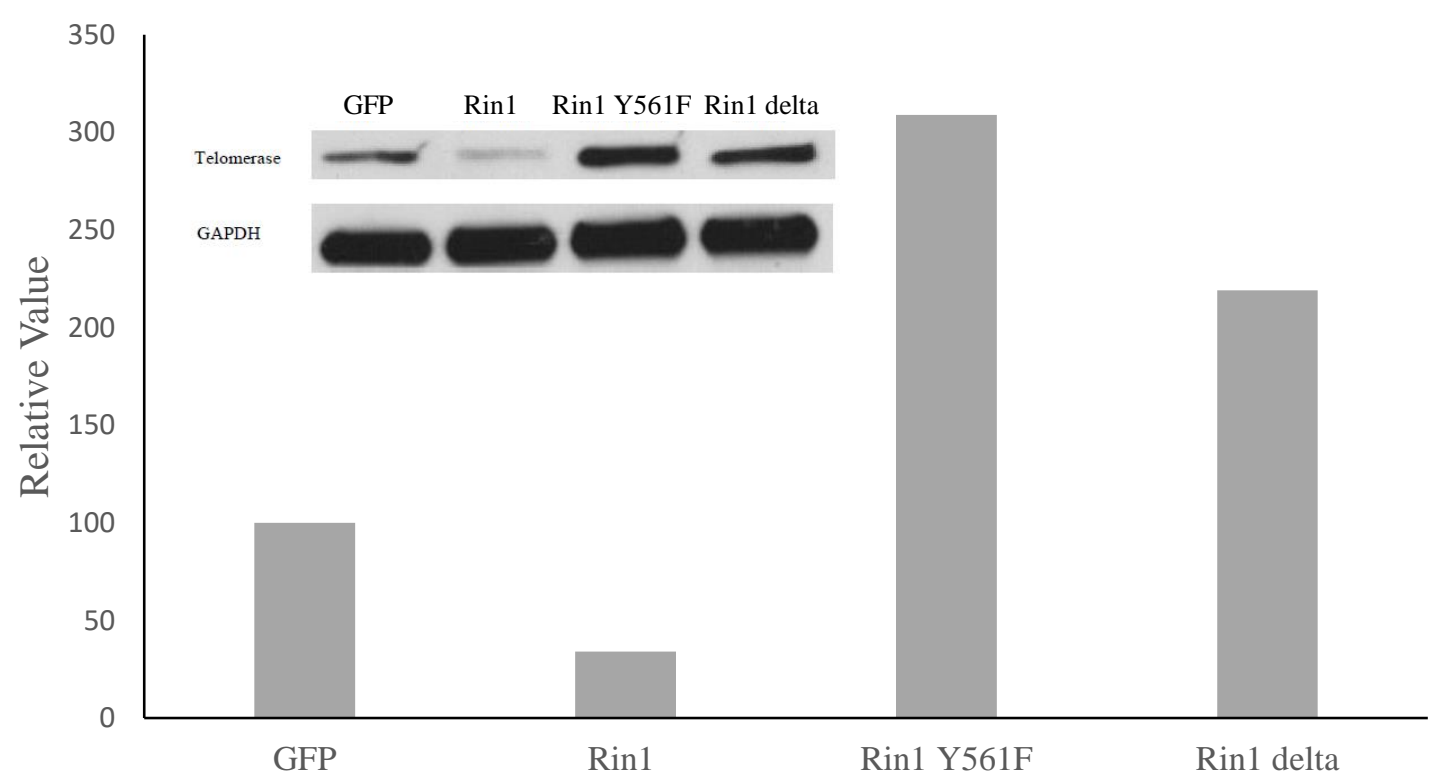

Figure 3.7: Effect of Rin 1 constructs on telomerase protein expression in MDA-MB 231 cells. Cells were stimulated with $50 \mathrm{ng} / \mathrm{mL}$ of IGF-1 for 24 hours. Values represent the percent increase normalized to GFP cells. 


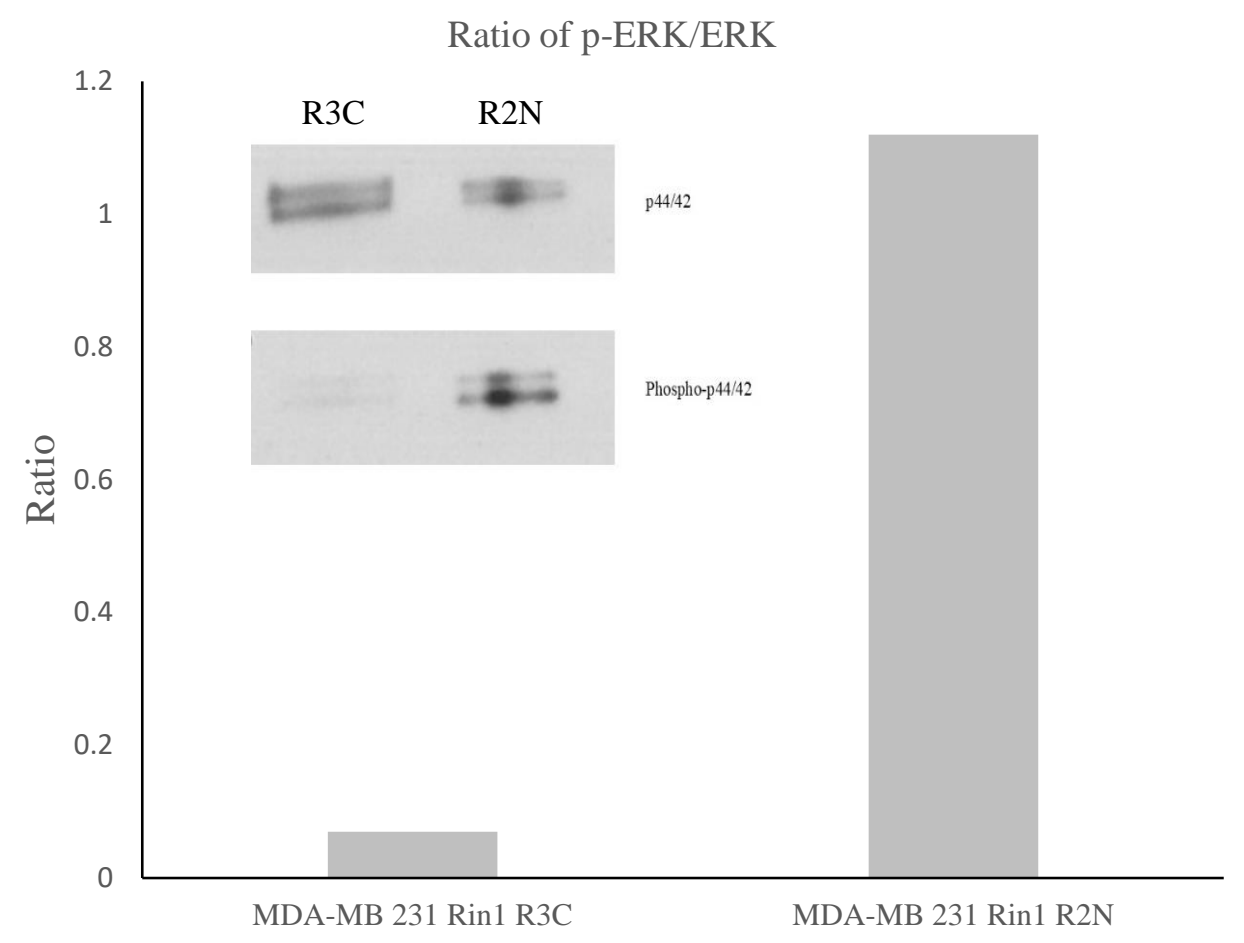

Figure 3.8: Effect of Rin1 amino (R2N) and carboxyl (R3C) terminal regions on the ratio of p-ERK/ERK in MDA-MB 231 GFP cells following IGF-1 stimulation (100 $\mathrm{ng} / \mathrm{mL}$ ) for 24 hours. 


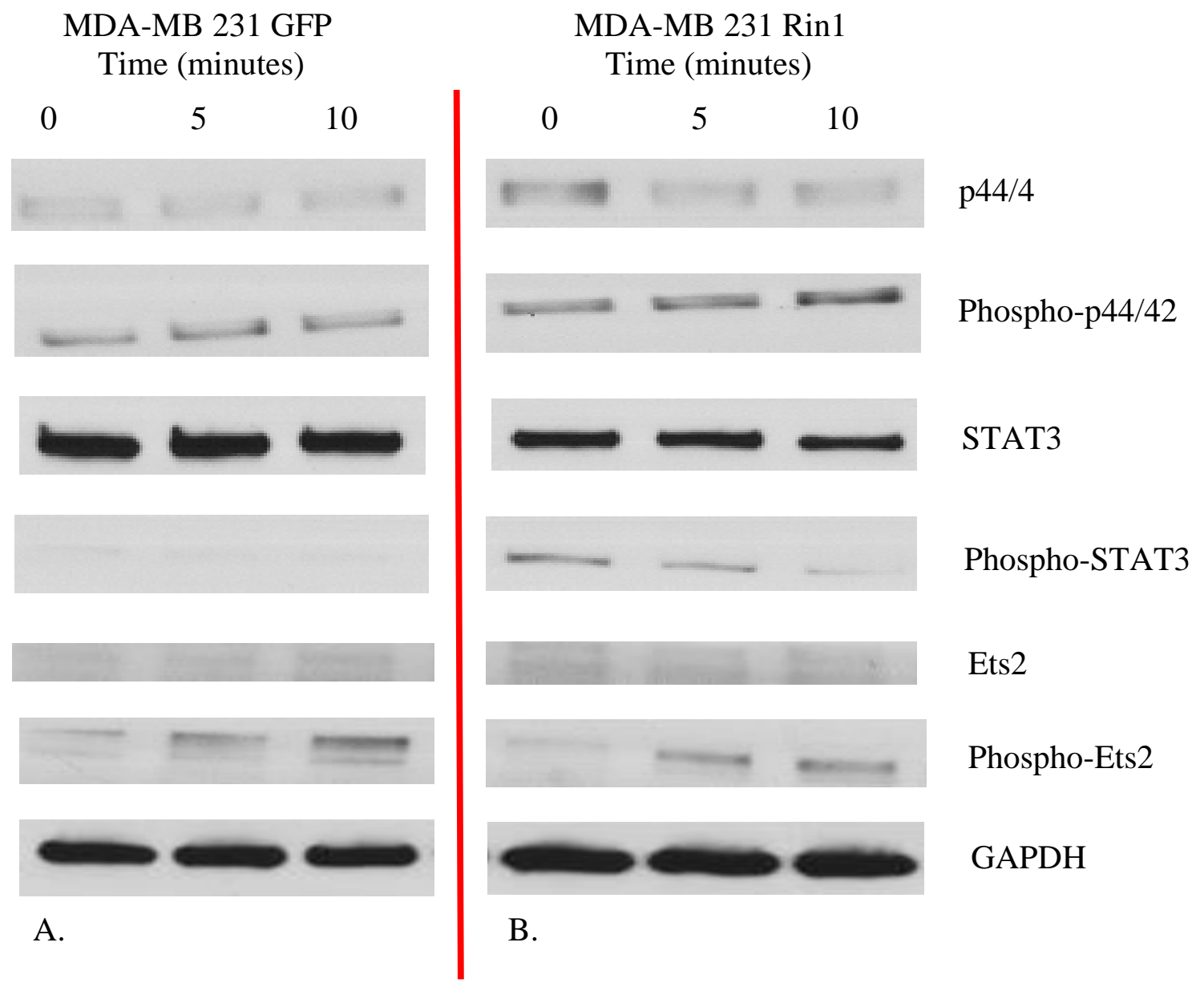

Figure 3.9: Western blot image of the effect of Rin1 on the expression and phosphorylation status of various proteins in the MDA-MB 231 GFP and Rin 1 cell lines following IGF-1 induction $(25 \mathrm{ng} / \mathrm{mL}$ ) for the times indicated (minutes). 


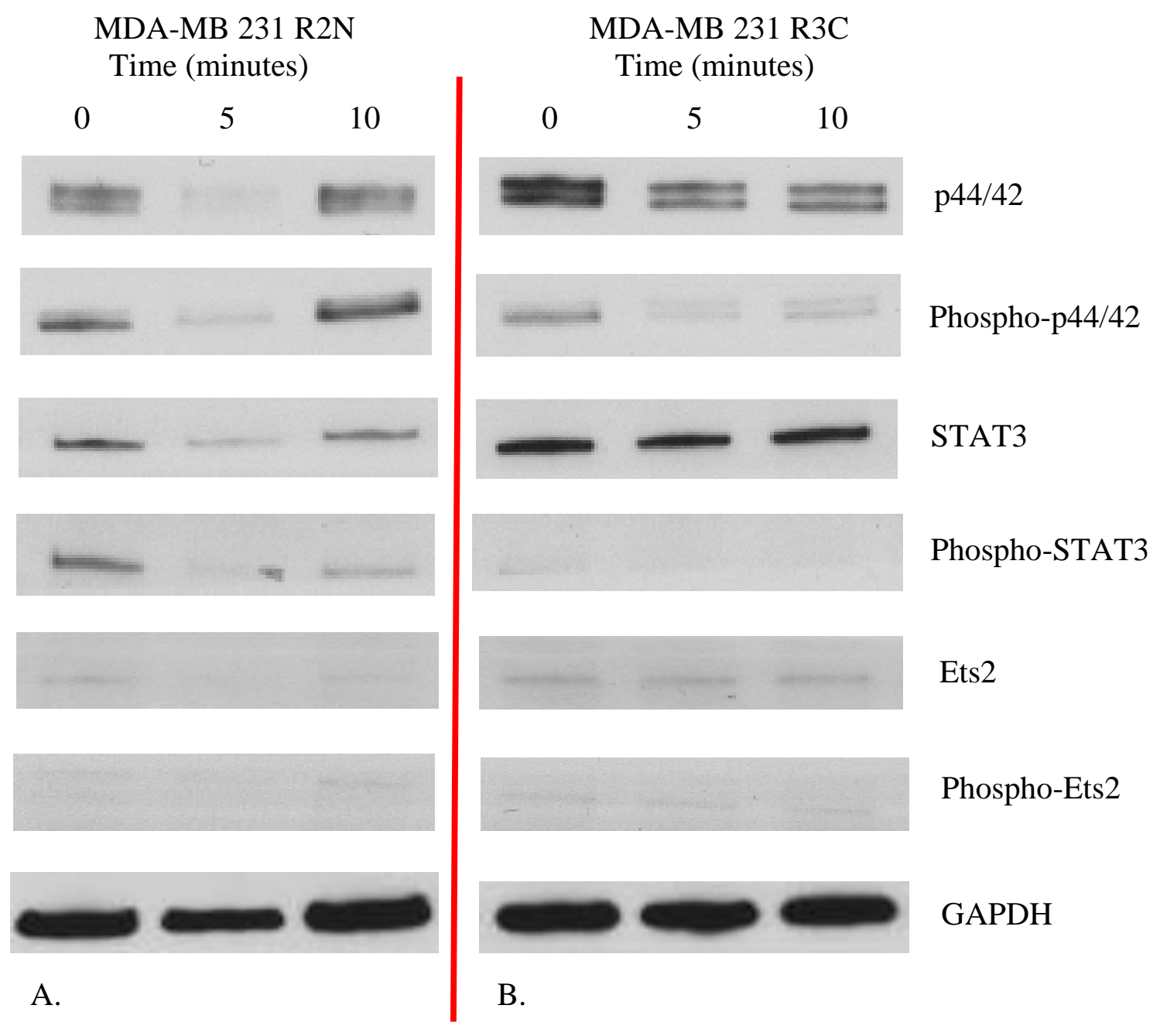

Figure 3.10: Western blot image of the effect of Rin1 on the expression and phosphorylation status of various proteins in the MDA-MB 231 amino (R2N) and carboxyl (R3C) cell lines following IGF-1 induction $(25 \mathrm{ng} / \mathrm{mL})$ for the times indicated (minutes). 


\begin{tabular}{|c|c|}
\hline \multicolumn{2}{|c|}{ MDA-MB 231 Telomerase Activity of Rin1 Constructs } \\
\hline & Average Ct Value \pm Standard Error \\
\hline Telomerase positive control & $25.03 \pm 0.20$ \\
\hline Telomerase negative control & $25.40 \pm 0.10$ \\
\hline GFP (+) IGF-1 & $25.94 \pm 0.10$ \\
\hline GFP (-) IGF-1 & $25.82 \pm 0.21$ \\
\hline Rin1 Y561F (+) IGF-1 & $25.88 \pm 0.16$ \\
\hline Rin1 Y561F (-) IGF-1 & $25.82 \pm 0.23$ \\
\hline Rin1 delta (+) IGF-1 & $25.92 \pm 0.13$ \\
\hline Rin1 delta (-) IGF-1 & $25.82 \pm 0.43$ \\
\hline Rin1 R2N (+) IGF-1 & $26.03 \pm 0.13$ \\
\hline Rin1 R2N (-) IGF-1 & $25.74 \pm 0.27$ \\
\hline Rin1 R3C (+) IGF-1 & $26.09 \pm 0.24$ \\
\hline Rin1 R3C (-) IGF-1 & \\
\hline Buffer & \\
\hline & \\
\hline & \\
\hline
\end{tabular}

Table 3.1: Cycle threshold values $(\mathrm{Ct})$ for the qPCR of telomerase activity in MDAMB 231 Rin 1 construct cell lines following stimulation with $100 \mathrm{ng} / \mathrm{mL}$ of IGF-1 (+) for 24 hours or no stimulation (-). Rin1 Y561F and Rin1 delta cells display 1.04 and 1.01 fold increases in telomerase activity compared to GFP cells. Rin1 R2N and Rin1 R3C cells display 1.06 and 1.11 fold decreases in telomerase activity compared to GFP cells. 
Effect of IGF-1 Concentration on Cellular Proliferation

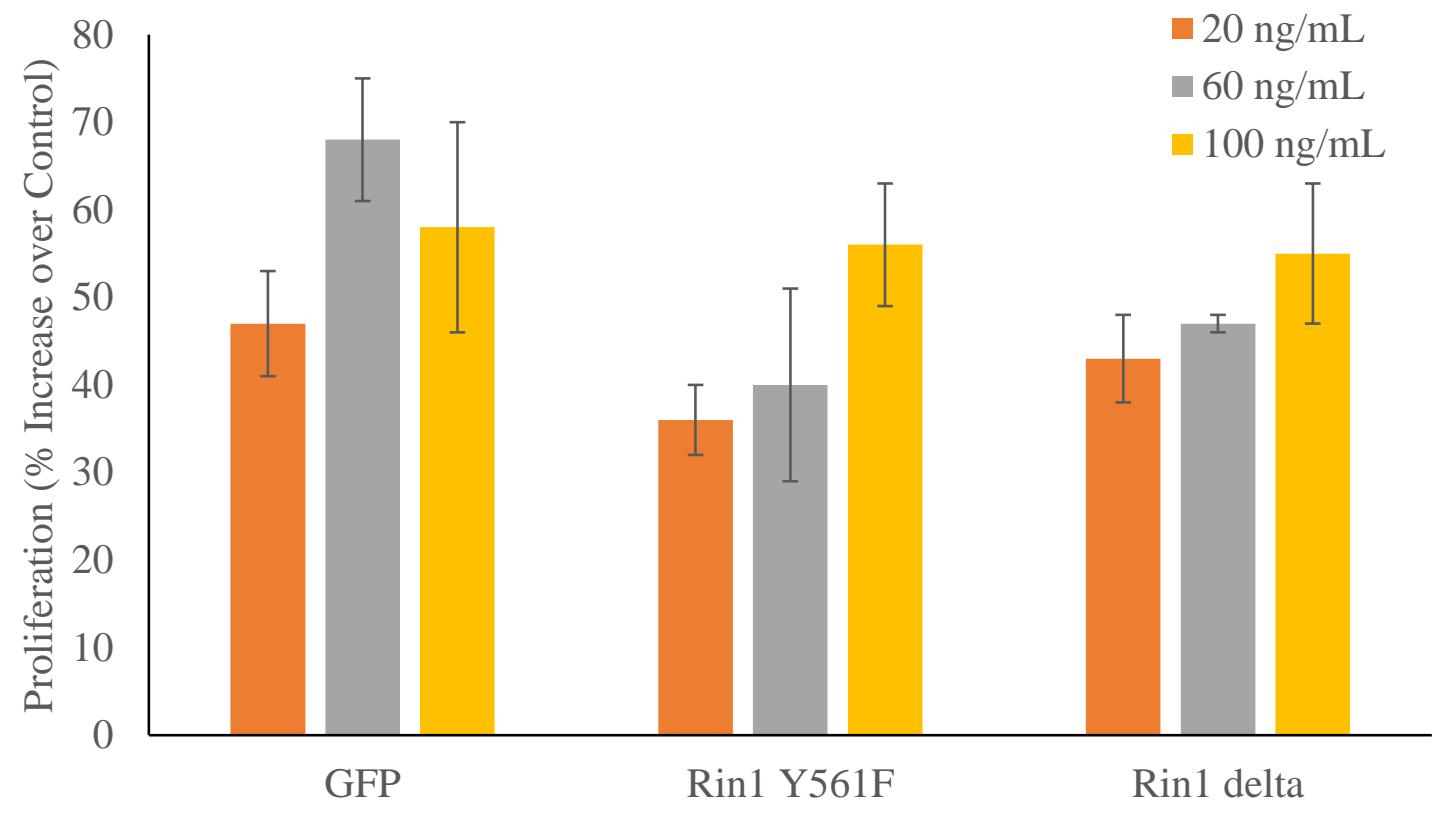

Figure 3.11: The effect of IGF-1 concentration on cellular proliferation in MDA-MB 231 cells expressing Rin1 Y561F or Rin1 delta. Values represent the percent increase in cellular proliferation over an unstimulated $(0 \mathrm{ng} / \mathrm{mL})$ control. All experiments were conducted in triplicate. Error bars represent standard error of the mean. 


\section{CHAPTER 4}

\section{The Effect of Rin1 on Telomerase Activity in Human Glioblastoma and Melanoma Cells}

\subsection{Introduction}

Small GTPases have recently come to the forefront of cell biology as important regulators of numerous cellular processes. Key cellular events such as extracellular signal transduction, proliferation, cell motility, and cytoskeletal rearrangements are all regulated to some extent by the action of small GTPases (Carvalho et al., 2015). Cellular small GTPases encompass a large and diverse group of proteins ranging from the heterotrimeric G-proteins associated with plasma membrane G-protein coupled receptors to the small and mostly cytosolic monomeric G-proteins (Csepanyi-Komi et al., 2012). The latter category of small GTPases has garnered much attention lately for their ability to function as molecular switches in the control of a variety of cellular processes, particularly those related to signal transduction, cell survival/proliferation, and cell motility.

The Ras superfamily of small monomeric GTPases are perhaps the best understood and most extensively studied in terms of their structure and function. The discovery of the Ras ( $\underline{\text { rat }}$ sarcoma) small GTPase almost twenty years ago has since led to the identification of a multitude of other small GTPases with similar structure and function to that of Ras. The Ras superfamily of small GTPases can be broadly divided into five distinct groups: Ras, Rho, Rab, Arf, and Ran (Carvalho et al., 2015). Each group within the Ras superfamily of small GTPases share a similar protein structure but can regulate separate and distinct cellular processes via their subcellular locations and interactions with downstream effectors (Bos et al., 2007). Additionally, the activity of each member of the Ras GTPase 
superfamily is controlled by a specific set of regulators known as guanine nucleotide exchange factors (GEFs) and GTPase activating proteins (GAPs).

In terms of their cellular roles, members of the Ras small GTPases are principally involved in extracellular signal transduction as well as in regulating cell differentiation and proliferation (Simanshu et al., 2017). The Arf and Rab family members are known to coordinate vesicle formation and intracellular vesicle trafficking as well as exocytosis, while Rho GTPases are known regulate cell motility and migration through control of cytoskeletal dynamics (Bos et al., 2007). The import and export of nuclear cargo as well as nuclear envelope and mitotic spindle formation are regulated by the Ran GTPases (Bos et al., 2007). Regardless of their individual roles, all Ras superfamily small GTPases have in common a highly conserved guanine nucleotide binding domain that consists of a six stranded $\beta$ sheet surrounded by five $\alpha$ helices (Cherfils and Zeghouf, 2013). Also contained within this domain are two switch regions referred to as Switch 1 (residues 30-38) and Switch 2 (residues 59-76) that change conformation when GTP is hydrolyzed as well as a phosphate binding loop (P-loop) (Carvalho et al., 2015). The two switch regions and the P-loop are involved in interactions with the phosphates of the bound guanine nucleotide and an essential magnesium ion that is necessary for strong binding of the nucleotide to the GTPase. Additionally, the carboxy terminus of most small GTPases is often modified by the attachment of prenyl groups such as farnesyl or geranylgeranyl that help to localize the G-protein to specific membranes. (Bos et al., 2007).

Most small GTPases are approximately $20-25 \mathrm{kDa}$ in size and all are capable of GTP hydrolysis (Csepanyi-Komi et al., 2012). Small GTPases function as molecular switches by alternating between an active and an inactive state. The binding of GDP to the protein 
favors the inactive state while the binding of GTP results in activation of the G-protein (Bos et al., 2007; Carvalho et al., 2015; Csepanyi-Komi et al., 2012). The exchange of GDP for GTP, and thus protein activation, is facilitated by multidomain proteins known as guanine nucleotide exchange factors (GEFs). Each member of the Ras superfamily of small GTPases is regulated by a specific group of GEFs with distinct structures (domains) and subcellular locations. For example, all GEFs for the Ras small GTPases contain a CDC25 homology domain along with a Ras exchange motif (REM) while most Arf family GEFs contain a Sec7 domain. The GEFs for Rho family GTPases typically contain a DH-PH domain while Vps9, Sec2, and Mss4 domains are characteristic of Rab family GEFs. The Ran GTPases are activated by the RCC1 GEFs (Bos et al., 2007; Cherfils and Zeghouf, 2013). Other domains involved in protein interaction such as $\mathrm{SH} 2, \mathrm{SH} 3, \mathrm{PH}$, and $\mathrm{C} 1$ are commonly found in GEFs besides the necessary GEF domains listed above (CsepanyiKomi et al., 2012). Upon activation by their respective GEFs, small GTPases then interact with unique sets of downstream effector proteins to perform the specific cellular functions associated with that particular family of small GTPases.

While GEFs are responsible for the activation of small GTPases by helping to exchange GDP for GTP, GTPase activating proteins (GAPs) promote the hydrolysis of GTP to GDP and thus result in G-protein inactivation. All small GTPases display intrinsic GTPase activity, however this activity is often very slow and not useful on a biological timescale. The GAPs therefore increase the rate of GTP hydrolysis by several orders of magnitude (Cherfils and Zeghouf, 2013). Like GEFs, most GAPs are multidomain proteins that interact with high specificity towards a particular family of small GTPases. 
The mechanisms of GEF activation and GAP inactivation are best understood for the Ras small GTPases. The activation of Ras typically occurs upon binding of growth factors such as epidermal growth factor (EGF) or insulin-like growth factor 1 (IGF-1) to their respective plasma membrane receptors. Growth factor activation of these receptor tyrosine kinases (RTKs) promotes the binding of adaptor proteins such as Grb2 to phospho-tyrosine residues on the activated RTK (Erijman and Shifman, 2016). A Ras GEF known as SOS binds to Grb2 and then activates Ras by stimulating the exchange of GDP for GTP. The GEF activity of SOS involves conformational changes in the two switch regions of Ras as well as in the P-loop. Specifically, the CDC25 GEF domain of SOS interacts with the switch 2 region and opens up the nucleotide binding site of Ras through the insertion of an $\alpha$ helix (Erijman and Shifman, 2016; Kiel et al., 2004). The interaction ultimately disturbs the magnesium ion within the nucleotide binding site of Ras and results in a decreased affinity of the G-protein for the phosphate groups of the bound GDP nucleotide. The GDP nucleotide is therefore displaced from Ras beginning with the release of the phosphate groups first while the incoming GTP nucleotide binds first through its base. High intracellular levels of GTP favor binding of GTP over re-binding of GDP once GDP is released from the G-protein (Erijman and Shifman, 2016; Kiel et al., 2004).

The inactivation of the Ras small GTPase occurs through the hydrolysis of the terminal $(\gamma)$ phosphate group of GTP through a mechanism involving stimulation by a Ras-GAP. The Ras-GAP promotes GTP hydrolysis through the insertion of a specific arginine residue (arginine finger) of the Ras-GAP that stabilizes the transition state by reducing the negative charge on the terminal phosphate group of GTP (Erijman and Shifman, 2016; Kiel et al., 2004). The actual hydrolysis of GTP is then accomplished by the coordination of a water 
molecule by a glutamine residue within Ras. The Ras-GAP helps to orient this glutamine residue for optimal GTP hydrolysis (Erijman and Shifman, 2016; Kiel et al., 2004).

While the general mechanisms of GEF and GAP activity are similar across the Ras superfamily of small GTPases, there do exist subtle differences in function depending on the particular G-protein family. For instance, the Vps9 domain of the Rab5 GEF known as Rin 1 activates Rab5 through the insertion of a group of $\alpha$ helices into the nucleotide binding site of Rab5. Here, an aspartate residue rather than an arginine residue, interacts with the P-loop of Rab5 to promote the exchange of GDP for GTP (Cherfils and Zeghouf, 2013; Barr and Lambright, 2010). Similarly, the Rabex5 GEF activates Rab21 by using its Vps9 domain to pry open the switch 1 region and then inserts an aspartate residue to interfere with the magnesium and phosphate binding sites (Barr and Lambright, 2010). Second messenger molecules such as cAMP are also known to activate the Rap small GTPases involved in cell adhesion. cAMP directly activates the Rap GEF Epac1 as well as protein kinase A (PKA), which in turn can phosphorylate Rap1 on its C-terminus and affect its subcellular localization. In another point of divergence with Ras, the RapGAP itself provides an asparagine residue to coordinate the attacking water molecule rather than the glutamine residue provided by Ras (Gloerich and Bos, 2011).

A growing body of evidence also implicates small GTPases in certain aspects of tumorigenesis (McFarlin et al., 2003). One of the defining hallmarks of most malignant cancers is the ability to spread or metastasize to new locations within the body and establish new tumors at sites distant from the primary tumor. In order for metastasis to occur, cancer cells must acquire an increasingly mobile phenotype that can lead to cell migration. The series of changes in cell morphology that accompany increased cell motility are often 
referred to as the epithelial to mesenchymal transition (EMT). The EMT is characterized by changes in epithelial cell polarity and loss of contact with neighboring cells. The Rho small GTPases figure prominently in the EMT given their normal roles of regulating cytoskeletal dynamics (Jansen et al., 2018).

The Rho family of small GTPases is quite large and can be broadly divided into three main subfamilies (Rho, Rac, and Cdc42) with each consisting of various related members. The most extensively studied and best understood Rho G-proteins are RhoA, Rac1, and Cdc42 (Wertheimer et al., 2012). Like other small, monomeric GTPases, Rho protein activity is regulated by GEFs and GAPs. Most Rho GEFs contain a Dbl homology (DH) domain where the GEF activity resides and a pleckstrin homology $(\mathrm{PH})$ domain that is capable of binding the membrane phospho-inositol lipid PI(3,4,5)P3. The binding of RhoGEF to PI(3,4,5)P3 following RTK or GPCR activation releases the autoinhibition of the DH domain. The RhoGEF is then free to activate a specific Rho G-protein and thus a specific group of downstream effectors that control rearrangement of the actin cytoskeleton (Hanna and El-Sibai, 2013). Unlike most RhoGAPs that stimulate GTP hydrolysis, the Rac1 GAP known as IQGAP1 is unusual in that it binds to GTP-Rac1 but does not exhibit GAP activity. Instead, IQGAP1 appears to interfere with the stability of adherens junctions by possibly interacting with $\beta$-catenin (Jansen et al., 2018).

The Rho dependent control of cell migration is illustrated in the regulation of focal adhesion formation by focal adhesion kinase (FAK). Focal adhesions are defined by interactions of integrins with ECM proteins and are typically found at the leading edge of migrating cells. In a proposed model of focal adhesion regulation, FAK binds to and phosphorylates the p190RhoGAP. This phosphorylation step activates the p190RhoGAP 
and results in the inhibition of RhoA (Tomar and Schlaepfer, 2009). A push/pull mechanism of cell movement occurs at the leading edge of the cell in which a reciprocal relationship between RhoA and Rac1 is the driving force. Elevated RhoA activity (pull/increased contractility) can inhibit Rac1 activity whereas decreased RhoA activity (push/decreased contractility) permits increased Rac1 activity. FAK mediated phosphorylation of the p190RhoGEF known as Rgnef activates RhoA and thus balances the p190RhoGAP activity as well as the migratory push/pull dynamic (Tomar and Schlaepfer, 2009).

The antagonistic relationship between RhoA and Rac1 is further illustrated in the type of cell movement governed by each protein. The RhoA activity tends to promote a cytoskeletal organization that results in a more generalized, amoeboid type of movement needed for cells to move through the ECM. In contrast, Rac1 activity is associated with a more directed type of movement such as lamellipodia formation (Kutys and Yamada, 2014; Parri and Chiarugi, 2010). In this example, increased Rac1 activity leads to the generation of reactive oxygen species (ROS) that oxidize and inactivate the low molecular weight protein tyrosine phosphatase (LMW-PTP) responsible for p190RhoGAP regulation. As a consequence, the p190RhoGAP remains phosphorylated and in an active state capable of downregulating RhoA activity through enhanced GTP hydrolysis (Parri and Chiarugi, 2010). The Rac1 activity also negatively influences RhoA activity through the downstream WAVE-2 effector complex. Conversely, low ROS levels activate LMW-PTP and therefore inactivate p190RhoGAP through its dephosphorylation. As a result, RhoA activity increases and begins to inhibit Rac1 through activation of the Rac1GAP known as ARHGAP2 (Parri and Chiarugi, 2010). 
The Rac1 activity is often very high in breast cancer cells and further underscores the importance of Rac GTPases in the malignant transformation of cells. The RacGEF P-Rex1 has been identified as an important activator of Rac1 and its expression is frequently upregulated in many breast cancers. The R-Rex1 is activated by signal inputs received from both the ErbB family of tyrosine kinase receptors as well as from GPCRs such as CXCR4 (Sosa et al., 2010). In the case of ErbB transactivation of CXCR4, the $\beta \gamma$ subunit of CXCR4 activates $\mathrm{PI} 3 \mathrm{~K} \gamma$ which in turn produces $\mathrm{PI}(3,4,5) \mathrm{P} 3$ at the plasma membrane. The P-Rex1 is then activated upon binding of its $\mathrm{PH}$ domain to $\mathrm{PI}(3,4,5) \mathrm{P} 3$ and consequently promotes Rac1 activation (Wertheimer et al., 2012). Additionally, Dillon et al. (2015) report that PRex1 may be able to create a positive feedback loop where P-Rex1 can activate the IGF-1 receptor as well as PI3K/AKT and MAPK signaling.

Inhibition of PI3K has also been shown to reduce MAPK signaling in breast cancer cells overexpressing HER2 (ErbB2) or expressing a constitutively active mutant form of PI3K. Here, PI3K inhibition suppresses Rac1 activation and thus limits the Rac1 mediated activation of its p21 protein activated kinase (PAK) effector. As a consequence, this subsequently reduces c-RAF1 activity and ultimately leads to lowered MAPK activity (Ebi et al., 2013). Hyperactive Rac3, like Rac1, is also associated with persistent p21 PAK and c-Jun N-terminal kinase (JNK) activation in certain breast cancer cell lines (Mira et al., 2000). In breast cancer cells overexpressing the IGF-1 receptor, knockdown of Rac1 greatly reduced resistance to the Trastuzumab anti-HER2 monoclonal antibody (Zhao et al., 2011).

Inappropriate regulation of small GTPases by GEFs and/or GAPs is a common mechanism in tumorigenesis. This is true for most members of the Ras superfamily of small 
G-proteins, but especially true for the Rho family of G-proteins where gain-of-function or loss-of-function mutations in GEFs or GAPs can lead to increased cell motility. There are however examples of where the Rho protein itself may be mutated and thus contribute to cancer development, such as the Rac1 P29L mutation observed in certain cases of melanoma. The activating mutation affects the switch 1 region and may help to stabilize the binding of GTP (Alan and Lundquist, 2013).

Unlike other members of the Ras superfamily, Ras proteins themselves are typically mutated with high frequency in a majority of cancers. The three major isoforms of Ras (KRas, N-Ras, and H-Ras) contain activating mutations in $15 \%$ to $30 \%$ of all cancers (Alan and Lundquist, 2013). Most of these mutations occur at positions 12, 13, and 61 and interfere with the ability of a RasGAP to stimulate GTP hydrolysis. Mutations at positions 12 and 13 specifically block the insertion of the RasGAP arginine finger while a mutation at position 61 interferes with the coordination of the water molecule needed for GTP hydrolysis (Simanshu et al., 2017).

In addition to the common mutations directly affecting Ras itself, mutational loss of RasGAP function can also contribute significantly to tumor development. For instance, loss of the RasGAP known as neurofibrimin is common in glioblastomas and contributes to the development of neurofibromatosis type 1 disease (Vigil et al., 2010). Mutational loss of another RasGAP called RHEB (Ras homology enriched in brain) is associated with a disorder known as tuberous sclerosis complex. Functional Rheb consists of a complex of two proteins known as tuberin and harmartin that are encoded by the TSC1 and TSC2 genes respectively (Vigil et al., 2010). Mutation of either gene can result in constitutive activation of the Rheb small GTPase and its effector mTORC1, with mTORC1 activity being strongly 
linked to increased cell growth and proliferation. The Rheb GAP activity is normally regulated by inactivation of tuberin through an Akt mediated phosphorylation. Therefore, mutations that activate PI3K or inactivate PTEN are also associated with oncogenic Rheb activation (Vigil et al., 2010). Similarly, the Rag small GTPases can also regulate mTORC1 activity through the sensing of cellular amino acid concentrations. Loss of the GATOR1 RagGAP results in hyperactive mTORC1 activity that is insensitive to amino acid depletion (Bar-Peled et al., 2013).

Epigenetic regulation of RasGAPs has recently been identified as an important mechanism in tumor formation. As an example, overexpression of the histone methyltransferase EZH2 subunit of the polycomb repressor complex 2 (PRC2) in prostate cancer cells results in transcriptional silencing of the DAB2IP RasGAP gene. The loss of DAB2IP RasGAP may represent a critical step in prostate cancer development and subsequent metastasis (Maertens and Cichowski, 2014). Epigenetic silencing of the RASAL2 RasGAP promoter via methylation has also been observed in certain metastatic breast cancer cell lines exhibiting reduced RASAL2 expression (Maertens and Cichowski, 2014; McLaughlin et al., 2013). The importance of RasGAPs in the regulation of Ras activity and tumor formation is highlighted in hepatocellular carcinoma cells. Here, transcriptional silencing via promoter methylation of either NF1, DAB2IP, or RASAL2 results in hyperactivity of wild type Ras and increased tumor aggressiveness (Calvisi et al., 2011).

In summary, the Ras superfamily of small GTPases mediate a number of important cellular processes related to cell survival and proliferation. Events such as signal transduction, cytoskeletal dynamics, and cell motility are all regulated by small G-proteins. 
Direct mutation of small GTPases or mutations in the GEFs/GAPs that regulate their activity are commonplace in many cancers. Further research specifically into the mechanisms of small GTPase regulation will undoubtedly reveal novel therapeutic approaches for cancer treatment.

\subsection{Results}

The U87 and U118 MG human glioblastoma cell lines overexpressing GFP and Rin1 were virally transduced (Figure 4.1) and selected for stable GFP and Rin1 expression (Figure 4.2) as described in 4.4, Creation of Cell Lines. The Yusik melanoma cell lines overexpressing GFP and Rin1 were also created and selected for stable GFP and Rin1 expression as described in 4.4, Creation of Cell Lines. Light micrograph images of the U87 MG human glioblastoma cell lines expressing GFP or Rin1 reveal stark differences in cellular morphology and growth patterns. The U87 MG cells that overexpress GFP (endogenous Rin1 levels) appear to grow more quickly and form distinct, irregularly clumped tumors (Figure 4.3, A.) when compared to cells expressing Rin1 (Figure 4.3, B.). The cells that express Rin1 tend to have a steady growth rate and are more evenly dispersed across the surface of the culture dish (Figure 4.3, B.).

Both the U87 and U118 MG cell lines displayed appreciable telomerase activity following IGF-1 stimulation (100 ng/mL) for 24 hours. With respect to the GFP cells, the U87 MG Rin1 cells displayed an increase in telomerase activity of 1.3 fold (Table 4.1). Similarly, a 1.1 fold increase in telomerase activity was observed for the U118 MG Rin1 cells when compared to the GFP cells (Table 4.2). Although a similar trend in telomerase activity following IGF-1 induction was observed for the U87 and U118 MG human 
glioblastoma cell lines, the average $\mathrm{Ct}$ values for the respective Rin1 cells differed by approximately 2 cycles, 26.13 versus 28.03 (Tables 4.1 and 4.2).

The Yusik human melanoma cell line was also utilized to investigate the effect of Rin1 expression on telomerase activity. Here, the Rin1 cells displayed a 1.49 fold decrease in telomerase activity when compared to the GFP cells (Table 4.3). The decrease in telomerase activity for Yusik melanoma cells overexpressing Rin 1 closely mirrors that observed in the MDA-MB 231 Rin1 breast cancer cells (Table 2.2).

\subsection{Discussion}

The effect of Rin1 on telomerase activity was examined in two human glioblastoma cell lines, U87 and U118 MG, and one human melanoma cell line, Yusik, in order to clarify if the influence of Rin1 on telomerase activity is cell type specific. The telomerase activity profiles for the MDA-MB 231 Rin1 breast cancer and Yusik Rin1 melanoma cell lines were remarkably similar. Overexpression of Rin1 reduced telomerase activity in each of these cell lines in comparison to the corresponding GFP expressing cells. Overexpression of Rin1 resulted in an approximate 1.50 fold reduction in telomerase activity for both cell lines following IGF-1 induction (Tables 2.2 and 4.3). The competition of Rin1 with RAF1 for binding to activated Ras may partially explain this result. The decrease in telomerase activity observed for the MDA-MB 231 Rin1 cells is particularly interesting given that the cell line contains a constitutively active mutant form of Ras; therefore, overexpression of Rin1 in these cells appears to effectively counter the heightened signaling through the MAPK pathway. Similarly, a mutation in the BRAF gene (V600E) is present in the Yusik melanoma cell line and contributes significantly to malignancy in these cells through a constitutively active form of BRAF. Surprisingly, the ability of Rin1 to attenuate MAPK 
signaling, and thus telomerase activity, in these cells was unexpected given that BRAF is constitutively active and not dependent upon competition with Rin1 to be activated by Ras.

The ability of Rin 1 modulate telomerase activity through MAPK signaling is largely dependent on the particular isoform of Rin1 expressed and the cell type it is expressed in. A potential tumor suppressive role for Rin 1 is supported by its effect on telomerase activity in the human MDA-MB 231 breast cancer and human Yusik melanoma cell lines, while an oncogenic effect is apparent in the U87 and U118 MG human glioblastoma cell lines. A comparison of light micrograph images of U87 MG cells expressing either GFP or Rin1 reveals a more even, steady growth pattern for cells overexpressing Rin1 versus the more clumped, tumorigenic appearance of the control (endogenous Rin1) GFP expressing cells (Figure 4.3, A. and B.). The observed differences in growth pattern and rate may be partly attributed to increased telomerase activity in the cells overexpressing Rin1. In fact, telomerase activity in the U87 MG Rin1 cells was 1.3 fold greater than in the corresponding GFP cells (Table 4.1). Additionally, a 1.1 fold increase in telomerase activity over the GFP cells was observed for the U118 MG Rin1 cells (Table 4.2). The heightened tumorigenicity of the U87 MG Rin1 cells is further evidenced by an average Ct value that is roughly 2 cycles lower than that of the corresponding U118 MG Rin1 cells.

In contrast to the MDA-MB 231 breast cancer and Yusik melanoma cell lines, Rin1 appears to promote telomerase activity in both the U87 and U118 MG glioblastoma cell lines. The exact mechanisms underlying cell specific differences in the effect of Rin1 on telomerase activity are unclear and remain an area for future research. 


\subsection{Materials and Methods}

\section{Creation of Cell Lines}

The U87 MG and U118 MG human glioblastoma cell lines as well as the Yusik human melanoma cell line stably expressing GFP and Rin1, were created using the same protocol as described previously in 2.4, Creation of Cell Lines. However, these cell lines were selected using a puromycin concentration of $1 \mu \mathrm{g} / \mathrm{mL}$ to account for the increased sensitivity of these cells to puromycin.

\section{Telomerase Activity Assay}

The human U87 MG and U118 MG glioblastoma cell lines as well as the human Yusik melanoma cell line expressing GFP and Rin1 were serum starved for 24 hours at $37^{\circ} \mathrm{C}$ and $5 \% \mathrm{CO}_{2}$ on a 12 well plate at a density of $1.0 \times 10^{5}$ cells per well in $1 \mathrm{~mL}$ of serum free growth media. All samples for each of the cell types were plated in duplicate. After 24 hours, $100 \mathrm{ng} / \mathrm{mL}$ of IGF-1 was added to half of the total samples- one well per cell type. The other half of the samples did not receive IGF-1 treatment. The samples were then incubated for another 24 hours, after which time the culture media in each well was removed and the cells were subsequently prepared for the telomeric repeat amplification protocol (TRAP) assay as per the manufacturer's instructions and as previously described in 2.4, Telomerase Activity Assay.

\section{Western Blotting}

Western blotting analysis of the U87 MG, U118 MG, and Yusik cell lines to confirm the expression of GFP and Rin1 was performed in the same manner as described previously in 2.4, Western Blotting. 


\subsection{References}

Alan, J. K., \& Lundquist, E. A. (2013). Mutationally activated rho GTPases in cancer. Small GTPases, 4(3), 159-163.

Barr, F., \& Lambright, D. (2010). Rab GEFs and GAPs. Current Opinion in Cell Biology, 22(4), 461-470.

Bar-Peled, L., Chantranupong, L., Cherniack, A., Chen, W., Ottina, K., Grabiner, C., \& Sabatini, D. (2013). A tumor suppressor complex with GAP activity for the rag GTPases that signal amino acid sufficiency to mTORC1. Science, 340(6136), 11001106.

Bos, J. L., Rehmann, H., \& Wittinghofer, A. (2007). GEFs and GAPs: Critical elements in the control of small G proteins. Cell, 129(5), 865-877.

Calvisi, D., Ladu, S., Conner, E., Seo, D., Jer-Tsong, H., Factor, V., \& Thorgeirsson, S. (2011). Inactivation of ras GTPase-activating proteins promotes unrestrained activity of wild-type ras in human liver cancer. Journal of Hepatology, 54(2), 311-319.

Carvalho, A. T. P., Szeler, K., Vavitsas, K., Åqvist, J., \& Kamerlin, S. C. L. (2015). Modeling the mechanisms of biological GTP hydrolysis. Archives of Biochemistry and Biophysics, 582(SI), 80-90.

Cherfils, J. \& Zeghouf, M. (2013). Regulation of small GTPases by GEFs, GAPs, and GDIs. Physiological Reviews, 93(1), 269-309.

Csépányi-Kömi, R., Lévay, M., \& Ligeti, E. (2012). Small G proteins and their regulators in cellular signalling. Molecular and Cellular Endocrinology, 353(1-2), 10-20.

Dillon, L., Bean, J., Yang, W., Shee, K., Symonds, L., Balko, J., \& Miller, T. (2015). PREX1 creates a positive feedback loop to activate growth factor receptor, PI3K/AKT and MEK/ERK signaling in breast cancer. Oncogene, 34(30), 3968-3976.

Ebi, H., Costa, C., Faber, A., Nishtala, M., Kotani, H., Juric, D., \& Engelman, J. (2013). $\mathrm{PI} 3 \mathrm{~K}$ regulates MEK/ERK signaling in breast cancer via the rac-GEF, $\mathrm{P}$ Rex1. Proceedings of the National Academy of Sciences of the United States of America, 110(52), 21124-21129.

Erijman, A. and Shifman, J. (2016). RAS/Effector interactions from structural and biophysical perspective. Mini-reviews in Medicinal Chemistry, 16, 370-375.

Gloerich, M., Johannes L. (2011). Regulating rap small G-proteins in time and space. Trends in Cell Biology, 21(10), 615-623.

Hanna, S., \& El-Sibai, M. (2013). Signaling networks of rho GTPases in cell motility. Cellular Signalling, 25(10), 1955-1961.

Jansen, S., Gosens, R., Wieland, T., \& Schmidt., M. (2018). Pharmacology and therapeutics (1. ed. ed.). Philadelphia, Pa: Saunders Elsevier. 
Kiel, C., Serrano, L., \& Herrmann, C. (2004). A detailed thermodynamic analysis of Ras/Effector complex interfaces. Journal of Molecular Biology, 340, 1039-1058.

Kutys, M. \& Yamada, M. (2014). An extracellular-matrix-specific GEF-GAP interaction regulates rho GTPase crosstalk for 3D collagen migration. Nature Cell Biology, 16(9), 909-917.

Maertens, O., \& Cichowski, K. (2014). An expanding role for RAS GTPase activating proteins (RAS GAPs) in cancer. Advances in Biological Regulation, 55, 1-14.

McFarlin, D., Lindstrom, M., \& Gould, M. (2003). Affinity with Raf is sufficient for Ras to efficiently induce rat mammary carcinomas. Carcinogenesis, 24(1), 99-105.

McLaughlin, S., Olsen, S., Dake, B., De Raedt, T., Lim, E., Bronson, R., \& Cichowski, K. (2013). The RasGAP gene, RASAL2, is a tumor and metastasis suppressor. Cancer Cell, 24(3), 365-378.

Mira, J., Benard, V., Groffen, J., Sanders, L., \& Knaus, U. (2000). Endogenous, hyperactive Rac3 controls proliferation of breast cancer cells by a p21-activated kinase-dependent pathway. Proceedings of the National Academy of Sciences of the United States of America, 97(1), 185-189.

Parri, M., \& Chiarugi, P. (2010). Rac and rho GTPases in cancer cell motility control. Cell Communication and Signaling : CCS, 8(1), 23.

Simanshu, D. K., Nissley, D. V., \& McCormick, F. (2017). RAS proteins and their regulators in human disease. Cell, 170(1), 17-33.

Sosa, M. S., Lopez-Haber, C., Yang, C., Wang, H., Lemmon, M. A., Busillo, J. M., \& Kazanietz, M. G. (2010). Identification of the rac-GEF P-Rex1 as an essential mediator of ErbB signaling in breast cancer. Molecular Cell, 40(6), 877-892. doi:10.1016/j.molcel.2010.11.029

Tomar, A., \& Schlaepfer. (2009). Focal adhesion kinase: Switching between GAPs and GEFs in the regulation of cell motility. Current Opinion in Cell Biology, 21(5), 676683.

Vigil, D., Rossman, K. L., Der, C. J., \& Cherfils, J. (2010). Ras superfamily GEFs and GAPs: Validated and tractable targets for cancer therapy? Nature Reviews Cancer, 10(12), 842-857.

Wertheimer, E., Gutierrez-Uzquiza, A., Rosemblit, C., Lopez-Haber, C., Sosa, M. S., \& Kazanietz, M. G. (2012). Rac signaling in breast cancer: A tale of GEFs and GAPs. Cellular Signalling, 24(2), 353-362.

Zhao, Y., Wang, Z., Jiang, Y., \& Yang, C. (2011). Inactivation of Rac1 reduces Trastuzumab resistance in PTEN deficient and insulin-like growth factor I receptor overexpressing human breast cancer SKBR3 cells. Cancer Letters, 313, 54-63. 


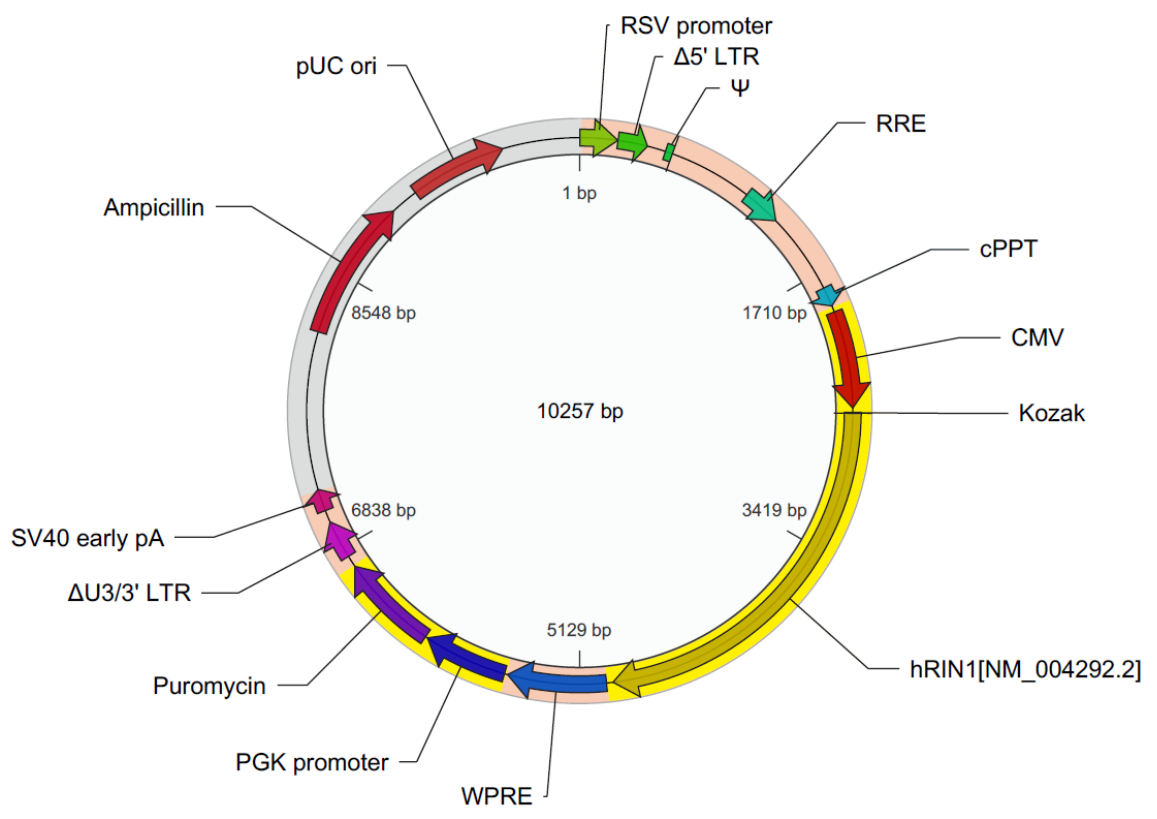

Figure 4.1: Lentiviral vector used to create the U87 MG, U118 MG, and Yusik cell lines expressing Rin1. The Rin 1 gene sequence is inserted directly downstream of the CMV promoter. An identical vector with the GFP gene sequence inserted was used to create the U87 MG, U118 MG, and Yusik cell lines expressing GFP. Also labeled are the various elements necessary for lentivirus production and cell selection. 


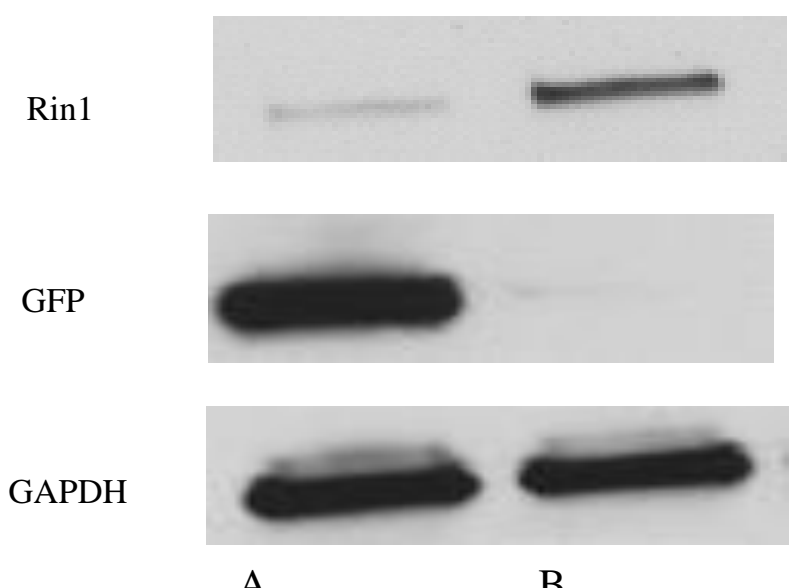

A.

B.

Figure 4.2: Western blot image of the A) U87 MG glioblastoma cell line expressing GFP and endogenous levels of Rin1. B) U87 MG glioblastoma cell line overexpressing Rin1.

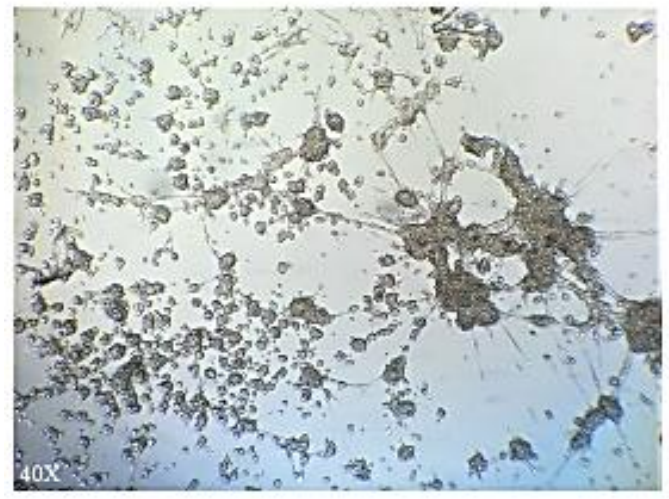

A.

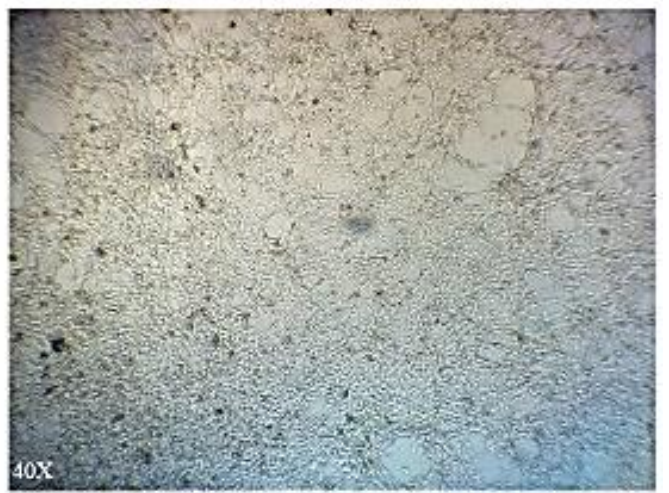

B.

Figure 4.3: Light micrograph images of the A) U87 MG GFP cells growing in a clumped, irregular pattern and B) U87 MG Rin1 cells growing in an even, uniform pattern. 


\begin{tabular}{|l|c|}
\hline \multicolumn{2}{|c|}{ U87 MG Telomerase Activity } \\
\hline & Average Ct Value \pm Standard Error \\
\hline Telomerase positive control & $25.16 \pm 0.27$ \\
\hline Telomerase negative control & $25.69 \pm 0.33$ \\
\hline GFP (+) IGF-1 & $26.47 \pm 0.44$ \\
\hline GFP (-) IGF-1 & $25.80 \pm 0.15$ \\
\hline Rin1 (+) IGF-1 & $26.13 \pm 0.04$ \\
\hline Rin1 (-) IGF-1 & $26.18 \pm 0.01$ \\
\hline Buffer & $26.19 \pm 0.49$ \\
\hline
\end{tabular}

$26.13-26.47=-0.34$
$2^{-(-0.34)}=1.3$
1.3 fold increase in telomerase activity compared to GFP cells.

Table 4.1: Cycle threshold values $(\mathrm{Ct})$ for the qPCR products of telomerase activity in U87 MG cell lines following stimulation with $100 \mathrm{ng} / \mathrm{mL}$ of IGF-1 (+) for 24 hours or no stimulation (-). All experiments were conducted in duplicate. 
Rin1

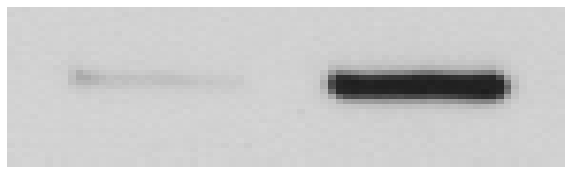

GFP

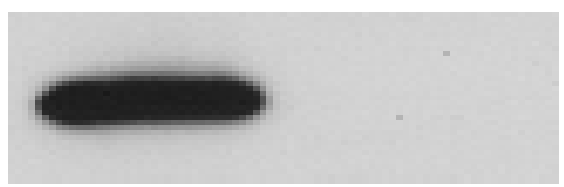

GAPDH

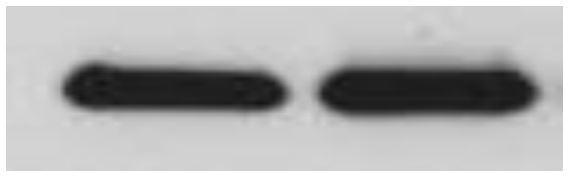

A.

B.

Figure 4.4: Western blot image of the A) U118 MG glioblastoma cell line expressing GFP and endogenous levels of Rin1. B) U118 MG glioblastoma cell line overexpressing Rin1. 


\begin{tabular}{|l|c|}
\hline \multicolumn{2}{|c|}{ U118 MG Telomerase Activity } \\
\hline & Average Ct Value \pm Standard Error \\
\hline Telomerase positive control & $27.34 \pm 0.14$ \\
\hline Telomerase negative control & $28.38 \pm 0.09$ \\
\hline GFP (+) IGF-1 & $28.20 \pm 0.16$ \\
\hline GFP (-) IGF-1 & $28.07 \pm 0.12$ \\
\hline Rin1 (+) IGF-1 & $28.03 \pm 0.02$ \\
\hline Rin1 (-) IGF-1 & $27.88 \pm 0.10$ \\
\hline Buffer & $28.20 \pm 0.18$ \\
\hline $\begin{array}{l}28.03-28.20=-0.17 \\
2\end{array}$ & \\
\hline 1.1 fold increase in telomerase activity compared to GFP cells.
\end{tabular}

Table 4.2: Cycle threshold values $(\mathrm{Ct})$ for the $\mathrm{qPCR}$ products of telomerase activity in U118 MG cell lines following stimulation with $100 \mathrm{ng} / \mathrm{mL}$ of IGF-1 (+) for 24 hours or no stimulation (-). All experiments were conducted in duplicate. 
Rin1

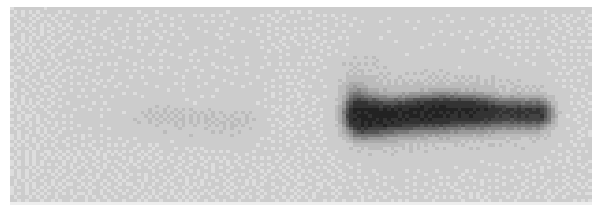

GFP

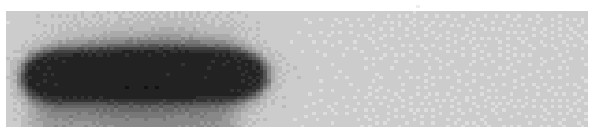

GAPDH

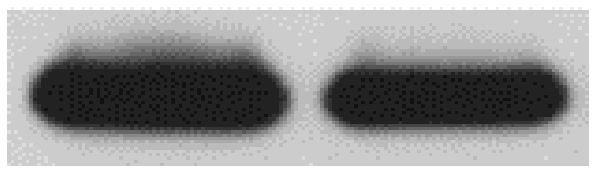

A.

B.

Figure 4.5: Western blot image of the A) Yusik melanoma cell line expressing GFP and endogenous levels of Rin1. B) Yusik melanoma cell line overexpressing Rin1. 


\begin{tabular}{|l|c|}
\hline \multicolumn{2}{|c|}{ Yusik Telomerase Activity } \\
\hline & Average Ct Value \pm Standard Error \\
\hline Telomerase positive control & $25.37 \pm 0.06$ \\
\hline Telomerase negative control & $25.99 \pm 0.26$ \\
\hline GFP (+) IGF-1 & $26.41 \pm 0.11$ \\
\hline GFP (-) IGF-1 & $26.35 \pm 0.13$ \\
\hline Rin1 (+) IGF-1 & $26.99 \pm 0.18$ \\
\hline Rin1 (-) IGF-1 & $26.73 \pm 0.24$ \\
\hline Buffer & $25.85 \pm 0.20$ \\
\hline
\end{tabular}

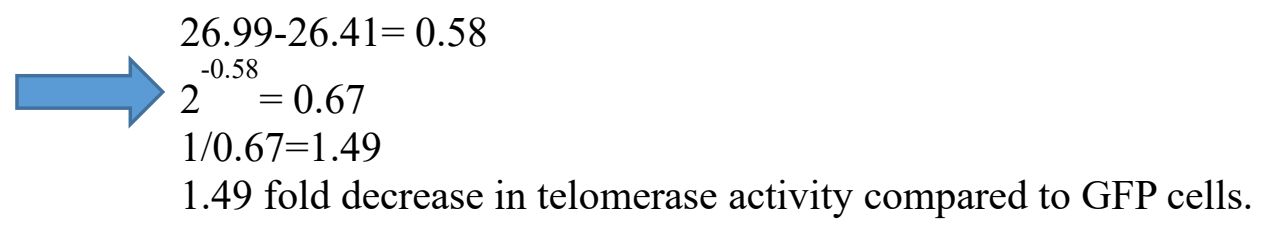

Table 4.3: Cycle threshold values $(\mathrm{Ct})$ for the $\mathrm{qPCR}$ products of telomerase activity in Yusik cell lines following stimulation with $100 \mathrm{ng} / \mathrm{mL}$ of IGF-1 (+) for 24 hours or no stimulation (-). All experiments were conducted in duplicate. 


\section{CHAPTER 5}

\section{Conclusions and Future Work}

The telomere regions of eukaryotic chromosomes are vital to the maintenance of genome stability. The gradual loss of telomeric DNA that occurs with each round of cell division acts like a molecular clock to set a finite limit on the number of times a population of cells can undergo cell division. The replicative limit is often reached when the telomeric DNA has become eroded to the point that genomic instability begins to occur as a consequence of chromosomal translocations and fusions. The replicative limit is bypassed in certain populations of cells that express the telomerase enzyme. In fact, activation of telomerase activity is a common mechanism by which many cancer cells acquire the immortal replicative phenotype that is frequently associated with malignant transformation.

The Ras and Rab interactor 1 protein (Rin1) may be able to regulate telomerase activity in cancer cells given its ability to modulate signaling through the MAPK pathway. Attenuation of MAPK is believed to occur as a result of the direct competition between Rin1 and RAF1 for binding to activated Ras. The hypothesis was examined in different cancer cell lines engineered to overexpress Rin1 or other Rin1 constructs such as Rin1 Y561F and Rin1 delta. Three specific aims were developed in order to determine if Rin1 can indeed function as a tumor suppressor in various cancer cell lines. The first aim of the research investigated the effect of Rin1 expression on cellular proliferation and viability as well as on telomerase gene expression and activity in three different human breast cell lines. The cell lines consisted of the tumorigenic and highly invasive MDA-MB 231 breast 
cancer cells, the tumorigenic but non-invasive MCF7 breast cancer cells, and the normal, non-tumorigenic/non-invasive MCF12A breast epithelial cells.

The Rin1 protein appears to function as a potential tumor suppressor in MDA-MB 231 breast cancer cells by downregulating telomerase activity. Reduced telomerase activity was also observed in the MCF7 cells. Downregulation of telomerase gene expression, a downstream target of the MAPK pathway, was observed in the MDA-MB 231 cells overexpressing Rin1. Similarly, the MCF-7 and MCF12A cells also exhibited reductions in telomerase gene expression as a consequence of Rin1 expression. Interestingly, Rin1 had the least suppressive effect on telomerase gene expression in the MDA-MB 231 cells while the strongest effect was observed in the MCF-12A cells. The result may be partially explained by the fact that the MDA-MB 231 cells harbor a constitutively active mutant form of Ras that may counteract the tumor suppressive effect of Rin1. The ability of Rin1 to suppress telomerase gene expression in the MCF7 cells is intermediate between that of the MDA-MB 231 and MCF-12A cells. The finding may be attributed to the tumorigenic but non-invasive nature of these cells.

In line with reduced telomerase gene expression and activity, MDA-MB 231 breast cancer cells overexpressing Rin1 generally display lower levels of cell proliferation and viability following IGF-1 stimulation when compared to non-transfected or GFP expressing cells. The result was also observed for the MCF-12A cells and to a lesser extent for the MCF7 cells. The MDA-MB 231 Rin1 cells grew more slowly and in a distinct clumped pattern in comparison to the GFP cells.

Growth factor receptors such as the insulin-like growth factor 1 receptor (IGF-1R) and the epidermal growth factor receptor (EGFR) play a prominent role in the development of 
breast cancer. The stimulatory effect of mitogens like IGF-1 or EGF on cellular proliferation and migration is well documented, as are the abnormalities in these receptors that often contribute to the unrestrained cell growth that is characteristic of so many different types of cancers. Besides growth factor receptors, the estrogen receptor (ER) also figures predominantly in breast cancer tumorigenesis. The signaling pathways activated by both types of receptors are an intense area of research and have shed light on the mechanisms of action by which various mitogens exert their oncogenic effects.

Therefore, in line with the importance of cell signaling to cancer cell growth and survival, the second aim of the research examined the effect of Rin1 expression on MAPK signaling and telomerase protein expression in MDA-MB 231 cells. Western blotting analysis of key MAPK pathway proteins following IGF-1 stimulation reveals reduced phosphorylation of the p-44/42 and Ets2 transcription factors. A strong inhibition of p44/42 phosphorylation as well as reduced telomerase activity were also observed in the MDA-MB 231 cells overexpressing the carboxyl terminal region of Rin1 containing the Ras association domain. Similarly, overexpression of Rin1 in MDA-MB 231 breast cancer cells reduces telomerase protein expression in comparison to cells expressing either the Rin1 Y561F and Rin1 delta constructs. The Y561F and delta forms of Rin1 do not bind as strongly to activated Ras when compared to the wild type form of Rin1. As such, they have a lesser ability to modulate MPAK signaling and thus display increased levels of telomerase activity following IGF-1 stimulation. Additionally, the Y561F and delta forms of Rin1 display increased levels of cellular proliferation across a range of IGF-1 concentrations when compared to cells expressing Rin1. Taken together, the data support a possible tumor suppressive role for Rin1 in MDA-MB 231 breast cancer cells. 
The third aim of the research sought to investigate the effect of Rin1 expression on telomerase activity in cell lines other than breast cells. The cell lines utilized were the human Yusik melanoma cells as well as the U87 MG and U118 MG human glioblastoma cells. The effect of Rin 1 on telomerase activity and thus its potential role as a tumor suppressor is not as clear in those cell lines as it is for the breast cell lines studied. In the Yusik melanoma cells that express a constitutively active mutant form of BRAF (V600E), Rin1 appears to function as a tumor suppressor by lowering telomerase activity in a manner very similar to that of the MDA-MB 231 breast cancer cells. However, its role in the U87 MG and U118 MG glioblastoma cells appears to be that of a possible oncogene through upregulation of telomerase activity. The results suggest that Rin1 is able to moderate telomerase activity in cancer cells in a manner that is largely dependent upon cell type.

A clearer picture of the potential tumor suppressor role of Rin1 will be obtained through future experimentation in a few key areas. Firstly, while the current research focused on the full length Rin1 protein as well as the amino and carboxyl terminal regions of Rin1, it would be beneficial to investigate what effect, if any, other domains of Rin1 might have on cellular proliferation/viability, telomerase gene and protein expression, and telomerase activity. For instance, the Vps9 domain of Rin1 is a known GEF for the Rab5 protein involved in the sorting of early endocytic vesicles. Could overexpression of the Vps9 domain or mutants of this specific domain affect signaling in other cancer cells besides MDA-MB 231 cells?

Another critical area for future research would be to determine if Rin1 or specific domains of Rin1 can influence the expression and/or phosphorylation status of MAPK signaling proteins in cell lines other than the MDA-MB 231 cells. Specifically, the carboxyl 
terminal region of Rin1 is a potent inhibitor of the phosphorylation of certain MAPK signaling proteins and can reduce telomerase activity. Would the same effects be observed in different cancer cell lines as well as normal cell lines? Also, what effect might the Rin1 Y561F and delta constructs have on telomerase gene expression and activity in cell lines other than the MDA-MB 231 cells? Lastly, the effect of Rin1 expression on telomerase gene expression and activity in response to stimulation by other growth factors such as EGF should also be examined.

In summary, the research findings indicate a novel molecular mechanism of action for Rin1 in specific cancer cell lines. Future experimentation to further elucidate the potential tumor suppressive role for Rin1 is needed and will expand upon the findings reported here. 
VITA

PATRIC HAMBLETON

EDUCATION and EXPERIENCE

2006

M.S., Biomedical Science

Barry University

Miami Shores, FL

2005

Master of Public Health

University of Miami

Coral Gables, FL

2003

B.S., Biology, minor in Chemistry

University of Miami

Coral Gables, FL

2018

Biomedical Research Initiative grant, NIGMS RISE R25 GM061347, \$3,000

2014-present

Academic Advisor, FIU

2006-2014

Science Faculty, Gulliver Preparatory School

\section{PRESENTATIONS}

Regulation of Telomerase Activity and Proliferation by Rin1 Upon IGF-1 Stimulation. American Society for Cell Biology annual meeting, San Diego, CA, December 2018

Regulation of Telomerase Activity by Rab5 Guanine Nucleotide Exchange Factors. Florida International University Biosymposium, Miami, FL, February 2018 